\title{
Upgrading low-quality feeds for tilapia by enzyme and probiotic supplementation
}

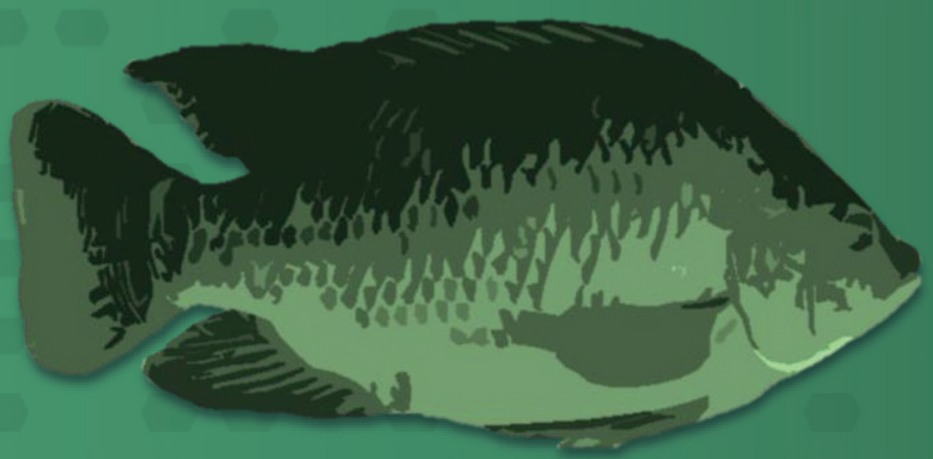

Roel M. Maas 


\section{Propositions}

1. The currently used phosphorus requirement for Nile tilapia needs to be reevaluated.

(this thesis)

2. Phytase has more potential than carbohydrases to improve the nutritional value of feeds in Nile tilapia.

(this thesis)

3. Peer reviewed journals should stimulate publication of studies with nonsignificant results to prevent publication bias.

4. Insects should not be used as a protein source in animal feed.

5. Teachers should stimulate students to go on adventure in the wilderness as it improves self-efficacy and reduces stress.

6. Education is a key factor in reducing the pressure of population growth on climate change.

7. Deep-frying fish is a culinary crime.

Propositions belonging to the thesis, entitled

Upgrading low-quality feeds for tilapia by enzyme and probiotic supplementation

Roel M. Maas

Wageningen, 5 February 2021 


\section{Upgrading low-quality feeds for tilapia by enzyme and probiotic supplementation}

Roel M. Maas 


\section{Thesis committee}

\section{Promotors}

Dr J.W. Schrama

Associate professor, Aquaculture and Fisheries

Wageningen University \& Research

Dr M.C.J. Verdegem

Associate professor, Aquaculture and Fisheries

Wageningen University \& Research

\section{Other members}

Prof. Dr W.H. Hendriks, Wageningen University \& Research

Prof. Dr M. Øverland, Norwegian University of Life Sciences, Norway

Dr A.J.P. Philip, Institute of Marine Research, Norway

Dr S. Brugman, Wageningen University \& Research

This research was conducted under the auspices of the Graduate School Wageningen Institute of Animal Sciences. 


\title{
Upgrading low-quality feeds for tilapia by enzyme and probiotic supplementation
}

\author{
Roel M. Maas
}

Thesis

submitted in fulfilment of the requirements for the degree of doctor at Wageningen University

by the authority of the Rector Magnificus,

Prof. Dr A.P.J. Mol,

in the presence of the

Thesis Committee appointed by the Academic Board

to be defended in public on

Friday 5 February 2021

at 4 p.m. in the Aula. 
Roel M. Maas

Upgrading low-quality feeds for tilapia by enzyme and probiotic supplementation,

228 pages.

PhD thesis, Wageningen University, Wageningen, The Netherlands (2021)

With references, with summaries in English and in Dutch

ISBN: 978-94-6395-626-0

DOI: $10.18174 / 535342$ 
Voor mijn ouders 



\section{Table of contents}

Chapter 1 General introduction

9

Chapter 2 Carbohydrate utilisation by tilapia: a meta-analytic 21 approach

Chapter 3 Effect of non-starch polysaccharide composition and enzyme supplementation on growth performance and nutrient digestibility in Nile tilapia

Chapter 4 Effect of exogenous enzymes (phytase and xylanase) supplementation on nutrient digestibility and growth performance of Nile tilapia fed different quality diets

Chapter 5 The effect of phytase, xylanase and their combination on growth performance and nutrient utilisation in Nile tilapia

Chapter 6 Effects and interactions between, phytase, xylanase and $\beta$ glucanase on growth performance and nutrient digestibility in Nile tilapia

Chapter 7 Effect of enzymes, probiotics and their combination on growth performance and nutrient utilisation in Nile tilapia

Chapter 8 Exogenous enzymes and probiotics alter digestion kinetics and microbial interactions in Nile tilapia gut

Chapter 9 General discussion

$\mathbf{R}$

References

$\mathbf{S}$

Summaries

211

A About the author 


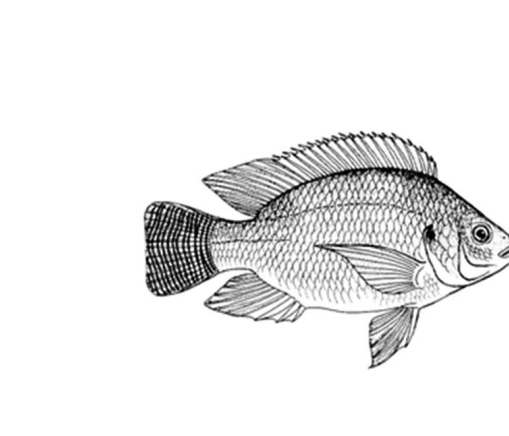




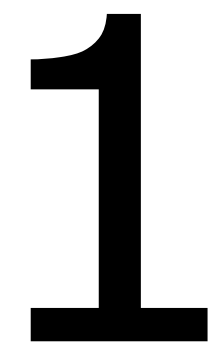

General introduction 
The aquaculture sector underwent rapid expansion during the last decades, growing annually by $4.7 \%$ between 2007 and 2018. In contrast, the landings from capture fisheries have been rather stable since the 1990s. In 2016 aquaculture overtook capture fisheries as the main source of fish for human consumption, providing approximately $7 \%$ of our total protein intake. Aquaculture will continue to expand, and become increasingly important, in face of the growing demand for protein from a rising human population. It is estimated that $59 \%$ of the fish consumed in 2030 will originate from aquaculture (52\% in 2018) (FAO, 2020; Tacon and Metian, 2015).

\section{Aquafeeds}

Finfish and crustaceans make up approximately $78 \%$ of total aquaculture (animal) production. Aquaculture of carnivorous fish has always required specialist feeds, whereas in the past, many herbivorous or omnivorous fish species (i.e. grass carp and tilapia) were kept in extensive systems, where no feed was provided. In 2012, it was estimated that $70 \%$ of fish and crustacean aquaculture was dependent on specialist feeds (Tacon and Metian, 2015). Due to intensification of aquaculture production, the percentage of fish not fed commercial diets is declining (Fry et al., 2016; FAO, 2020). An increase in aquaculture production, together with an increase in the proportion of fish fed commercial diets, has meant that global aquafeed production has soared. Aquafeed production increased by $106 \%$ between 2000 and 2008 and was projected to increase by a further $124 \%$ between 2008 and 2020 (Tacon et al., 2011; Tacon and Metian, 2015; Fry et al., 2016). The production of fishmeal and fish-oil, the conventional protein and fat source in aquaculture feeds, has remained stable in absolute amounts, and is expected to remain so in the near future. As aquaculture is the largest consumer of fishmeal and fish-oil, their limited production has led to the increased use of alternative ingredients, mainly from terrestrial plants (Figure 1 ).

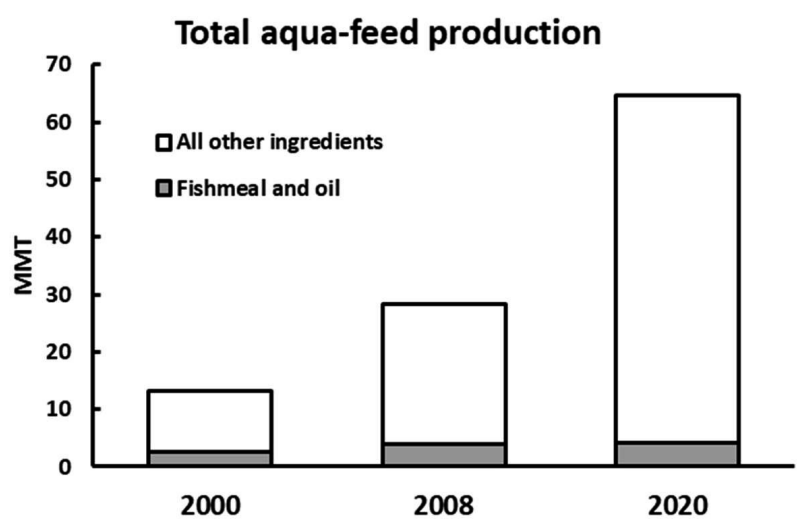

Figure 1 Growth and projected growth (2020) of the use of commercial aquaculture feed in million metric tons (MMT). Here, the absolute use of fishmeal and fish-oil remains stable, while the proportion of fish meal and oil in the diet declines (adapted from Fry et al., 2016). 
With the expected further growth of aquaculture and the increasing demand for aquafeeds, the trend for reducing levels of fishmeal and fish-oil in aquafeeds will continue. This will increase the pressure on novel alternative feed ingredients, like insects, microalgae and macroalgae (Shepherd and Jackson, 2013; Troell et al., 2014; Tacon and Metian, 2015; Fry et al., 2016).

\section{Anti-nutritional factors in aquafeeds}

With increasing levels of plant by- and co-products within aquafeeds over time, the level of anti-nutritional factors (ANF) in aquafeeds has increased, which reduced their nutritional value. ANFs have been defined as substances that directly, or indirectly, interfere with food utilisation and thus affect the health and productivity of animals (Makkar, 1993). There are a wide range of ANFs, including: protease inhibitors; saponins; tannins; phytates; lectins; alkaloids; antigenic compounds; antivitamins; cyanogens; oligosaccharides; and non-starch polysaccharides (NSP). NSP and phytate are probably the most common ANFs (Francis et al., 2001). The phosphorus (P) in phytate (phosphate) is bound to an inositol and not bioavailable to fish (Kumar et al., 2012). Generally, plant ingredients contain a mix of ANFs. The proportion and level of ANF is ingredientdependent and heavily influenced by the degree of processing. When processed into, for instance, protein concentrates and isolates, the carbohydrate fraction, including the hulls containing most of the ANFs, are removed. The quality of 'meals', like soybean meal and sunflower meal, depends on the amount of ground hulls added to the meal. The aim of this thesis was to reduce the negative effects, in formulated diets, that NSP and phytate have on nutrient utilisation and fish growth performance. The importance of improving the utilisation of the NSP and phytate content is given below.

\section{Carbohydrates}

Carbohydrates are often divided into starch, sugars and NSP. Starch and sugars are highly digestible, whereas NSP is considered to be indigestible to fish. NSP and dietary fibre are often used interchangeably, although the dietary fibre fraction, quantified as crude fibre, is only part of the NSP fraction. NSP are generally classified into three main classes; cellulose, hemicellulose (non-cellulosic polymers) and pectins (pectic polysaccharides) (Choct, 1997). Of the total dietary fraction, cellulose, hemicellulose and pectins make up the majority (90-95\%). The remainder are smaller fractions like fructan polysaccharides, lignin, oligosaccharides, gums and mucilages, which are often not considered to be part of the NSP fraction. All these fractions have the following similarities: they are structural components of plants and are thus found mainly in the cell wall; and they are of differing shapes and structures, making them hard to analyse (Sinha et al., 2011; Dhingra et al., 2012). Therefore, throughout this thesis the term NSP is used as a collective term to describe all the carbohydrates, excluding starch and sugars. This separation allows for the quantification of the total amount of NSP, by measuring the starch and sugar content. 
Although not considered essential for fish, carbohydrate is a cheap energy source. How well a fish can process carbohydrates is species specific (Wilson, 1994; Hemre et al., 2002). For example, in rainbow trout and barramundi the utilisation efficiency of digestible carbohydrate decreases with increasing levels of digestible carbohydrate intake; whereas this utilisation efficiency is constant (i.e. a linear relationship) in Nile tilapia and common carp (Schrama et al., 2018; Phan et al., 2019). It is commonly accepted that sufficient levels of easily digestible carbohydrates allow for protein sparing effects (Kaushik and de Oliva Teles, 1985; Krogdahl et al., 2005; Zheng et al., 2020). In general, omnivorous warm water fish can handle higher levels of plant ingredients than carnivorous cold-water fish. Therefore, omnivorous warm water fish show much higher rates of protein sparing (Wilson, 1994; Hemre et al., 1993; Krogdahl et al., 2005).

NSP is considered indigestible, as the enzymes needed to break down the bonds of the (often) long polysaccharides are scarce or non-existent in fish (Castillo and Gatlin, 2015; Krogdahl et al., 2015; Zheng et al., 2020). Starch is the major polysaccharide, stored as an energy reserve, in many plants. Starch is composed of glucose units linked by a-glycosidic bonds. Differences between the type of bonds (a vs. $\beta$ ), makes starch highly digestible and NSP indigestible. The assumption that NSP are indigestible, and thus of no nutritional value, is one of the major reasons why NSP are classified as ANF. In addition, increasing NSP levels can have a negative effect on nutrient digestibility in several ways. NSP may entrap nutrients in cell walls and organelles (Teuling et al., 2019); and it can have multiple negative effects in the fish gut, like the deconjugation of bile salts, changes in chyme viscosity, gut morphology, physiology and mucus layer. These changes may lead to reduced nutrient digestibility and thereby compromised growth (Choct, 1997; Choct and Kocher, 2000; Sinha et al., 2011; Staessen et al., 2020).

This thesis focusses on NSP, as it is the main part of the ANF. As NSP is a structural component of the cell wall of plants, it cannot be eliminated from aquafeeds when using plant ingredients; unless only starch and protein isolates and concentrates are used (i.e. purified ingredients). Although considered indigestible, a few studies have shown that tilapia can partially digest NSP, indicating the potential of NSP to be utilised for energy (Amirkolaie et al., 2005; Leenhouwers et al., 2007a; Haidar et al., 2016), most likely through fermentation. Despite these isolated studies, information about the degradation and fermentation of NSP in fish is limited. Besides being a potential energy source, improving the utilisation of NSP may reduce its associated negative effects, which include its adverse impact on the digestibility of macro-nutrients.

\section{Phytate}

Phytate is present in almost all seeds and is the major ( $P$ ) form in plant-derived feed ingredients (Ravindran et al., 1995). Replacing fishmeal with plant ingredients coincides with decreased bioavailability of dietary $P$ in fish feeds, since phytate bound $\mathrm{P}$ is poorly absorbed by fish. Like NSP, phytate bound $\mathrm{P}$ generally remains 
unavailable for fish, because of the lack of endogenous enzymes (phytase) to liberate phosphate from the inositol of the phytate molecule. In addition, phytate forms complexes with minerals and amino-acids, reducing their bioavailability and thus impacting growth (Pimentel-Rodrigues et al., 2007; Kumar et al., 2012).

For decades, $\mathrm{P}$ in aquaculture has been a hot topic, as poor bioavailability of $\mathrm{P}$ will lead to its increased discharge into the water. This can stimulate eutrophication in natural waters. In some areas, the effluent from fish farms is subject to strict legislation. This can restrict aquaculture production and/or affect its economic viability (Riche and Brown, 1996; Peukhuri, 2002; Mainstone and Parr, 2002). It is essential that sufficient levels of bioavailable $P$ are present in aquafeeds, as $P$ plays a fundamental role in the synthesis of ATP and nucleic acid, and the formation of bones and scales. $P$ deficiency can result in poor growth and deficient bone mineralisation, which in turn can lead to bone deformities. There have been many studies on $P$ in aquafeeds, due to its importance for growth and skeletal development, as well as the issues associated with its discharge (Boaventura et al., 1997; Suigiura and Ferraris, 2004; Fontagne et al., 2009). The requirement for low $\mathrm{P}$ levels in aquaculture effluents, is in conflict with the higher $\mathrm{P}$ levels required to sustain optimum growth; this means that the feed formulation has to be optimised to ensure the highest possible $P$ retention.

In aquafeeds that contain more plant-based ingredients, inorganic $\mathrm{P}$ needs to be supplemented in order to fulfil the $P$ requirements for growth. However, using $P$ from (mined) phosphate rock deposits is unsustainable, given that natural reserves of phosphate are limited (Prabhu et al., 2013; Obersteiner et al., 2013). By improving the bioavailability of phytate bound $P$ from plant ingredients, the dependency of aquaculture on inorganic mined $P$ sources can be reduced, thus improving its circularity. The optimal amount of $P$ in the diet is a combination of the $P$ requirement of the fish and the $P$ bioavailability of the diet. Diets with a low $P$ bioavailability require higher levels of $P$ to fulfil the $P$ requirements. Both lower dietary $\mathrm{P}$ levels, and a higher dietary $\mathrm{P}$ bioavailability will contribute to lower $\mathrm{P}$ levels in aquaculture effluent.

\section{Future trends in aquafeeds}

As aquaculture production continues to grow, the use and demand for plant ingredients in aquafeeds increases. Multiple crops are used in commercial aquafeeds like rapeseed, soybean, maize, or wheat. From the processed cropbased ingredients used, soybean meal is the most common (23\%) (Tacon et al., 2011; Fry et al., 2016). By 2050, the world population is expected to grow by almost 2 billion, reaching 9.7 billion people. The population of Africa and Asia, the two continents with the highest level of undernourishment, have the highest projected growth, at 52\% and 17\% (2015-2050), respectively (United Nations, 2015; Chouchane et al., 2018). The increasing world population and demand for food puts stress on food security. This is likely to lead to a shift towards the increased use of plant ingredients/products in aquafeeds that would not otherwise 
be directly consumed by humans. The most obvious example of a feed ingredient currently used in aquafeeds that could be increasingly used for direct human consumption, is soybean meal. Soybean meal is the most utilised protein source in farmed animal (including fish) production, due to its high nutritional value (Fry et al., 2016; Heuze et al., 2020). Currently only around $2 \%$ of soybean meal is used for soy flour and other products for human consumption, such as dairy substitutes (soy milk, soy cheese, etc.).

Consequently, the use of low quality ingredients will increase as they are less suitable for human consumption. Coinciding with increasing levels of low quality ingredients, the level of ANFs will increase, resulting in a lower feed utilisation efficiency and increased waste production. To which extent lower quality ingredients will, and can, be used in aquafeeds is species dependent. How well a fish can deal with increasing levels of (low quality) plant ingredients, and thus ANFs, is species specific and depends on factors like the gut physiology and morphology. A known example is the occurrence of enteritis in salmonids at high inclusion levels of soybean meal (Baeverfjord and Krogdahl, 1996; Urán et al., 2008). Moreover, the quality of the diet is partly dependent on the value of the fish, i.e. feeding high quality feeds to pangasius may not be profitable, whereas it is for salmon. Therefore, the increase in low quality co- and by-products, and thus ANF, is expected to be higher for low value omnivorous fish like tilapia and pangasius, compared to high value carnivorous fish like yellowtail kingfish and salmon.

\section{The use of feed additives}

Feed additives have been defined by the AAFCO (2000) as "An ingredient or combination of ingredients added to the basic feed mix or parts thereof to fulfil a specific need. Usually used in micro quantities and requiring careful handling and mixing". The range of feed additives in aquafeeds is very diverse, meaning multiple approaches can be used to improve the feed in different ways. Feed additives are often non-nutritive and are typically added to improve the feed quality and nutrient bioavailability, to reduce the negative effects of an ANF, or to improve fish health or disease resistance. The feed quality can be improved by adding binders, which improve the water stability of the pellet, but may affect the viscosity of the digesta and faeces. Antioxidants and preservatives are added to improve the shelf-life of feeds. Enzymes (discussed below) are used to improve the bioavailability of certain nutrients. Multiple additives have been reported to improve animal health and disease resistance, like probiotics (discussed below) and immunostimulants (i.e. $\beta$-glucans). Other additives neutralise the effect of specific ANFs through binding (i.e. mycotoxin binders) (Encarnação, 2016; Dawood et al., 2018). The list of current feed additives is extensive (yeasts, organic acids, phytogenics etc.), and novel feed additives will continue to be explored, extending the list of possibilities. 


\section{Enzymes}

There are four main groups of enzymes used as feed additives in aquafeeds; proteases, lipases, phytases and carbohydrases. These enzymes are used to improve the bioavailability of certain nutrients and/or to eliminate the presence of certain ANFs (phytate and NSP), leading to improved nutrient digestibility and growth (Encarnação, 2016; Zheng et al., 2020). Carbohydrases catalyse a reduction in the molecular weight of polymeric carbohydrates. Carbohydrases can be roughly divided into amylases (targeting starch) and NSP-degrading enzymes (targeting NSP). Among the NSP-degrading enzymes, there are a wide range, including; glucanases, xylanases, pectinases and cellulase. Like all enzymes, they will accommodate the catalysation of only one type of chemical reaction, for a specific substrate. The use of exogenous enzymes, including NSP-degrading enzymes, has been widely studied in pigs and poultry. Compared to other monogastric animals, studies on fish using phytase and NSP-degrading enzymes are limited. In these studies using exogenous enzymes in fish, a mix/cocktail of various enzymes is often used (proteases, lipases, amylases, etc.), in particular when using NSP-degrading enzymes (Sinha et al., 2011; Castillo and Gatlin, 2015; Zheng et al., 2020). When a mix of enzymes is used it is not possible to directly link observed effects on, for instance, nutrient digestibility, to the individual enzyme. Moreover, in pigs and poultry, multiple studies have evaluated the interaction between enzymes; showing that synergy occurs between enzymes (Juanpere et al., 2005; Tahir et al., 2005). Similarly, synergy between different types of enzymes in fish can be expected, but this has not yet been assessed.

In addition, although the main purpose of phytase is to improve $\mathrm{P}$ bioavailability, the $P$ bioavailability is often not measured when applying an enzyme cocktail containing phytase. Moreover, NSP digestibility has never been demonstrated with enzyme supplementation (including NSP-degrading enzymes). Therefore it remains unclear if, and to what extent, NSP digestibility can be enhanced by supplementing aquafeeds with enzymes.

\section{Probiotics}

Probiotics have been extensively investigated as possible feed additives over the last two decades. Generally, probiotics are used as an alternative to antibiotics. They can enhance immunity and disease resistance both on the inside and outside of host (fish). Probiotic bacteria can protect against pathogens by producing inhibitory substances, essential nutrients or enzymes; in this way they stimulate a beneficial immune response, or positively alter the microbial composition in the gastro-intestinal tract, gills, skin etc. (Gómez et al., 2008; Pérez et al., 2010). Studies investigating the use of probiotics often focus on measuring health-related parameters and performing challenge tests for disease resistance. Some studies on fish have shown improved growth performance with probiotics (Hai, 2015; Merrifield et al., 2010). Multiple studies have been performed, both on broilers (Apata, 2008; Mountzouris et al., 2010; Hossain, 2015) and pigs (Giang et al., 
2011; Lee et al., 2015; Lan et al., 2016), on the improvement of nutrient bioavailability with the use of probiotics. However, for fish, similar studies are limited; little is currently known about how probiotics can improve nutrient bioavailability of plant-based aquafeeds.

\section{Tilapia as a model species}

Nile tilapia was chosen as a model animal for this thesis. Tilapia is a major species group in freshwater fish aquaculture; second only in production to major carp. The aquaculture of Nile tilapia is fast expanding and is expected to exceed the production of grass and silver carp in the coming decade, thereby becoming the most cultured fish species by volume (Prabu et al., 2019; FAO 2020). Tilapia has, as a predominantly herbivorous warm-water fish with a long gut, the potential for gut fermentation (Bergman, 1990; Metzler-Zebeli et al., 2010). Moreover, a few studies have shown the ability of tilapia to partly digest NSP, which is linked to fermentation (Amirkolaie et al., 2005; Leenhouwers et al., 2007a; Haidar et al., 2016). This is important in order to test the hypothesis that exogenous enzymes can improve nutrient bioavailability and digestibility of, in particular, NSP and phytate. Tilapia is known to deal well with high levels of plant ingredients and carbohydrates in its diet, which allows for a varied diet composition (i.e. high levels of NSP and phytate). This also enables low quality feed ingredients to be tested; ingredients that would not otherwise be used for human consumption. Moreover, global fish production is dominated by major carps $(50 \%)$, tilapias $(10 \%)$ and catfishes $(8 \%)$. These omnivorous fish species all have a relatively low market value, therefore feeds need to be inexpensive in order to ensure profitability. However, less expensive diets come at the cost of quality; increasing levels of low quality ingredients, and thus ANFs, can be expected. In addition, much is known about the nutrition and nutritional requirements of tilapia, in comparison to major carps and catfish. This reasoning makes tilapia a suitable and obvious model species, to assess the potential of enzymes and probiotics to counteract the negative effects of NSP and phytate.

\section{Rationale}

The use of low quality ingredients for many fish species, including tilapia, will increase in the future, leading to increased dietary levels of ANF, including NSP and phytate. Both NSP and phytate are indigestible by fish, due to the lack of necessary enzymes to break down their bonds. NSP can be degraded by gut microbiota, leading to the production of volatile fatty acids (VFA). It is expected that phytase and NSP-degrading enzymes will improve the digestibility and bioavailability of NSP and $P$, reducing the associated negative effects of NSP and phytate. When NSP is broken down, more substrate will be available for VFA production. VFA can provide a source of energy for the fish and can have positive health effects (Bergman, 1990; Montagne et al., 2003). Healthy animals make better use of dietary nutrients and are less sensitive to disease, thus antibiotic use is decreased or even eliminated. Some commonly used dietary probiotics, including 
various Bacillus strains, metabolise VFA and might work better, over a longer period, in a VFA-rich gut.

Enzymes and probiotics are administered through the diet, therefore it is likely that diet-related factors will play a major role in their desired effect. However, in studies testing the effect of enzymes or probiotics, a single (basal) diet is generally used. Therefore, it remains unclear how diet-related factors may affect the efficacy of enzymes and probiotics. As enzymes are specific for certain substrates it can be expected that the composition of the targeted fractions, in this case NSP and phytate, may affect the response to dietary enzyme supplementation. It can also be expected that when the diet is of low quality (i.e. low digestibility), or when ANF are expressed more, there is a greater potential for improvement with enzyme and probiotic supplementation.

\section{Aim and outline of the thesis}

This thesis foresees the increasing importance of under-utilised crop waste in formulated aquafeeds and their positive contribution to the 'bio economy'. Indeed, one of the main goals in the context of circular food production is to formulate aquafeeds with improved digestibility, containing plant ingredients that would otherwise be wasted (FAO, 2020). This will allow for a more sustainable growth of aquaculture. The general aim of this thesis is to investigate whether the nutritional value of low quality diets can be improved, with the use of exogenous enzymes and probiotics, using tilapia as a model species. The leading questions addressed in this thesis are:

- Is tilapia able to "digest" and utilise NSP endogenously?

- Can dietary probiotics and enzymes (phytase and carbohydrases) supplementation improve nutrient digestibility and utilisation?

- Are there interactions between enzymes (mutually) and probiotics?

- Do diet-related factors (quality, levels of ANF, composition etc.) have an effect on the response to enzyme supplementation?

The objective of this research is to allow for the increased use of low-quality crop residues in aquafeeds, which will contribute to the development of circular food systems.

\section{Overview of chapters 2-9:}

Chapter 2: Assesses and quantifies whether tilapia can digest NSP, using metaanalysis of available data on nutrient digestibility in the literature. The metaanalysis assesses which factors (body weight, nutrient composition diet etc.) could influence NSP digestibility and how dietary NSP level and NSP digestibility could influence other factors, like nutrient digestibility and growth.

Chapters 3 and 4: Explores whether exogenous enzymes (phytase and xylanase) could improve NSP and P digestibility, and thereby enhance growth; and whether the impact of the enzymes is dependent on diet-related factors. Chapter 3 
investigates the effect of NSP composition and chapter 4, the effect of diet quality (dietary levels of NSP and phytate).

Chapter 5: Tests whether there is interaction (including synergy) between enzymes; in this case the enzymes phytase and xylanase. The main effect(s) of the individual enzymes (phytase and xylanase) is explored as well.

Chapter 6: Studies whether $\beta$-glucanase, another NSP-degrading enzyme, can effect growth and nutrient digestibility, and whether $\beta$-glucanase can have interactions with phytase and xylanase. Using phytase, xylanase, and phytasexylanase in combination again, checks for repeatability of effects observed in chapter 5.

Chapter 7: Tests whether probiotics could improve diet quality, by measuring nutrient utilisation and growth, and the interaction of probiotics with supplemented enzymes.

Chapter 8: Looks at the effect of enzymes and probiotics on the digestion kinetics and production of VFA (fermentation product of NSP), along the gastrointestinal tract, as well as impacts on microbiota in the distal gut.

Chapter 9: The general discussion. The main outcomes of this thesis are placed in the context of current insights on the use of enzyme (NSP-degrading and phytase) and probiotic supplements in aquafeeds, exploring how the quality of fish diets can be further improved. 


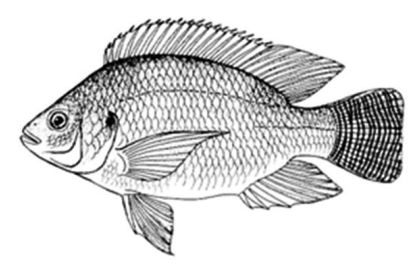




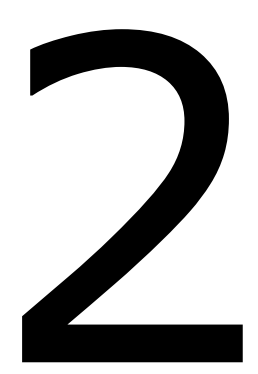

\section{Carbohydrate utilisation by tilapia: a meta-analytic approach}

Roel M. Maas, Marc C.J. Verdegem, Geert F. Wiegertjes,
Johan W. Schrama

Reviews in Aquaculture (2020), 12: 1851-1866 


\begin{abstract}
Currently, studies reporting carbohydrate, starch, and especially non-starch polysaccharides (NSP), digestibility in fish are scarce. Carbohydrate digestibility in the diet is largely dependent upon carbohydrate composition (starch vs. NSP). NSP are often considered to be indigestible and thus of no nutritional value. The present study reviews carbohydrates in fish feed, distinguishing between total carbohydrate, starch and NSP. Besides a qualitative approach, a meta-analysis was performed, compiling available data from digestibility studies on tilapia. Our meta-analysis confirms the negative effect of NSP on performance (FCR) and nutrient digestibility (crude protein, fat and energy). However, an average NSP digestibility of $24.3 \%$ was calculated in 95 cases. Out of these 95 cases, $88 \%$ of them showed a positive NSP digestibility. NSP digestibility showed to contribute to energy digestibility. The digestion of NSP in fish is associated with fermentation in the gut, producing beneficial volatile fatty acids which are rapidly absorbed by the colonic lumen. Therefore, in diet formulation, digestibility and thus energy originating from NSP should be taken into consideration because NSP contributes to the energy needs of fish, here tilapia. Besides being an energy source, specific types of NSP may have immune modulating and prebiotic effects and may be increasingly added to fish feed as modulators of fish health. We suggest that NSP is potentially (partly) digested by a wide range of fish species, especially by warm water species with a long gut adapted to feeding on plant matter, as these factors favour gut fermentation.
\end{abstract}




\section{Introduction}

Many studies have been performed with tilapia (Oreochromis spp.), comparing digestibility across a wide range of plant ingredients used as substitutes for dietary fishmeal and oil. In general, these studies use a reference diet with a particular percentage of plant ingredients, such as: soya bean meal; linseed meal; canola meal; cottonseed meal; sunflower meal; wheat middlings; corn gluten; rice bran; barley; rye etc. (Degani et al., 1997; Dong et al., 2010; Gaber, 2006; Köprücü and Özdemir, 2005: Obirikorang et al., 2015; Schneider et al., 2004; Sintayehu et al., 1996). In these studies, the focus is predominately on protein and fat digestibility, with little or no attention given to carbohydrate digestibility. Carbohydrates are the cheapest energy source for human, fish and other animals. Carbohydrate requirements have not been extensively investigated, compared to fat and protein requirements. It is commonly accepted that appropriate levels of carbohydrates should be incorporated in fish diets to improve the energy availability, albeit sparingly for carnivorous fish like trout and salmon, which are less well adapted to digest complex carbohydrates. Lack of carbohydrates in fish diets will increase the catabolism of protein and lipids (Wilson, 1994; Kim and Kaushik, 1992), while sufficient levels of easily digestible carbohydrates allow for protein sparing effects (Krogdahl et al., 2005; Hemre et al., 1993; Kaushik and de Oliva Teles, 1985).

The optimal level of dietary carbohydrates differs widely amongst fish species. In general, warm water and freshwater fish are known to utilise higher levels of carbohydrates than cold water or marine species (Wilson, 1994). Omnivorous fish can handle higher levels of carbohydrates than carnivorous fish, and show much higher rates of protein sparing (Krogdahl et al., 2005; Hemre et al., 1993; Hemre et al., 2002). A review by Wilson (1994) recommend a dietary digestible carbohydrate level of $40 \%$ for tilapia, whilst the optimum for marine, carnivorous and cold-water fish (i.e., Atlantic salmon, rainbow trout and plaice) is below $20 \%$. Besides differences in optimal dietary carbohydrate levels, the way fish utilise digestible carbohydrates differs between species. The energy utilisation efficiency of digestible carbohydrates in common carp and Nile tilapia is found to be constant (linear relationship), whereas for barramundi and rainbow trout, the utilisation efficiency decreases with increasing levels of digestible carbohydrate intake (Schrama et al., 2018; Phan et al., 2019). This suggests that some fish have a limited capacity to process carbohydrates.

The total carbohydrate digestibility in the diet is largely dependent on the carbohydrate composition. The total carbohydrate digestibility depends on the starch level, the molecular complexity of the carbohydrate source and total carbohydrate level in the diet (Stone, 2003). The starch fraction of the diet is considered highly digestible with apparent digestibility coefficients (ADC) of $90 \%$, up to $99 \%$ in Nile tilapia (Amirkolaie et al., 2006; Haidar et al., 2016; Leenhouwers et al., 2007a). The ADC of the total carbohydrate fraction has been found to be as low as 30-60\% (El-Saidy and Gaber, 2003; Deng et al., 2016) and as high as 80- 
90\% for Nile tilapia (Tran-Ngoc et al., 2016; Sintayehu et al., 1996). The large variation in the digestibility of the total carbohydrate fraction is mainly caused by differences in the total dietary fibre fraction.

The dietary fibre fraction consists of remnants of plant cells, which are non-starch polysaccharides (NSP), oligosaccharides, lignin and gums (Dhingra et al., 2012). The NSP forms the bulk of the total dietary fibre fraction. In this review, the term NSP is used to describe the total dietary fibre fraction, including oligosaccharides, lignin and gums. There is a wide range of NSP, differing in both characteristics and properties (i.e., solubility), whilst the amount and type of NSP differ amongst plant ingredients. NSP is generally classified in cellulose, non-cellulosic polymers (hemicellulose) and pectic polysaccharides. Cellulose is a complex polysaccharide, solely consisting of D-linked glucose units (links of 3000 or more). The noncellulosic polymers are divided into arabinoxylans, mixed-linked $\beta$-glucans, mannans, galactomannans and glucomannans. The pectic polysaccharides mainly consist of D-galacturonic acid (GaIA), which forms arabins, galactans and arabinogalactan (Choct, 1997; Sinha et al., 2011). NSP are considered indigestible by monogastric animals, as they lack enzymes such as $\beta$-glucanases and $\beta$ xylanases to break down the long polysaccharide chains. Therefore, NSP are often considered to be of little or no nutritional value (Sinha et al., 2011; Choct, 1997; Stone, 2003).

Although considered indigestible, a study by Leenhouwers et al. (2007a) found a digestibility of up to $24 \%$ in Nile tilapia for the NSP fraction when using barley as the main NSP source. Amirkolaie et al. (2005) also showed digestibility for purified cellulose and guar gum in Nile tilapia, with an ADC of 2.8 and $20.8 \%$ respectively. A more recent study using Nile tilapia by Haidar et al. (2016) showed a digestibility between $41 \%$ and $73 \%$, depending on the feeding level, for a diet enriched with dried distillers grains with solubles from wheat (DDGS).

\section{Aim}

The scarce information available shows large differences in carbohydrate and NSP digestibility in tilapia (Amirkolaie et al., 2006; Haidar et al., 2016; Leenhouwers et al., 2007a), suggesting that different factors can potentially affect the digestibility of carbohydrates and NSP. However, studies reporting carbohydrate, starch, and especially NSP digestibility are scarce, which makes general statements about their digestibility difficult. Considering the above, the present study reviews carbohydrates in fish feed, distinguishing between total carbohydrates, starch and NSP. Besides a qualitative approach, a meta-analysis was performed, compiling the available data from digestibility studies with tilapia; thus calculating the theoretical total carbohydrate, starch and NSP level in the diet, as well as the digestibility of the total carbohydrate fraction and NSP. The NSP level, carbohydrate and NSP digestibility were checked for correlations with, amongst others, feeding level, nutrient composition of the diet and digestibility of other nutrients. The qualitative and quantitative (meta-analyses) approaches are 
combined in this manuscript to improve the understanding of the role and nutritional value of carbohydrates in fish feed, focusing predominately on the NSP fraction. This manuscript describes the total carbohydrate, starch and NSP fraction in separate chapters, followed by concluding remarks.

\section{Methodology}

\section{Approach}

In order to have an overview and quantify the digestibility of total carbohydrates, starch, NSP and other nutrients, existing digestibility studies concerning tilapia were compiled. From here on $\mathrm{CHO}$ is used for the total carbohydrate fraction, being the dry matter minus the sum of the crude protein, fat and ash. Digestibility studies concerning tilapia were used, as several studies already showed the potential of tilapia to digest NSP. In addition, tilapia is widely used as a model species in digestibility studies, ensuring an adequate amount of data. Tilapia also has, as a herbivorous warm water fish with a long gut, the potential for gut fermentation (Bergman, 1990; Metzler-Zebeli et al., 2010).

Studies with common and red coloured Nile tilapia (Oreochromis niloticus (L.)), hybrid tilapia (Oreochromis niloticus x Oreochromis aureus) and Mozambique tilapia (Oreochromis mossambicus) were selected. Only studies in which $\mathrm{CHO}$ in the diet was given, or could be calculated from the reported dry matter (DM), crude protein, crude fat and ash or energy content, were considered. The $\mathrm{CHO}$ digestibility was calculated indirectly when not reported. The $\mathrm{CHO}$ digestibility was calculated from either 1 ) the mass balance using the reported ADC of DM, crude protein, fat and ash; or 2) the reported ADC of energy, crude protein and fat as described by Schrama et al. (2018). Studies were excluded when the $\mathrm{CHO}$ digestibility could not be calculated. Studies testing the digestibility under hypoxic conditions were also excluded. Only controls where fish were fed under normoxic conditions were used to exclude the effect of oxygen level on digestibility. In addition, studies in which enzymes or probiotics were incorporated in the diet were not included, as these can influence the endogenous nutrient digestibility (Castillo and Gatlin, 2015; Hai, 2015). Besides the nutrient composition of the diets ( $\mathrm{g} / \mathrm{kg}$ $D M)$ and the ADC; the feeding level, average weight of the fish at the start and end, duration of the feeding trial, FCR, specific growth rate (SGR) and how the diets were manufactured (extrusion, steam pelleting) were registered. These factors can potentially affect or be affected by, the NSP level and CHO and NSP digestibility.

In order to calculate a theoretical NSP digestibility, the NSP level and starch level in the diet had to be determined. This was done by reformulating all the diets with the use of the Centraal Veevoederbureau (CVB) 2016 database. The total CHO in the diet being known, as well as the ratio between NSP and starch, allowed for calculation of the total amount of NSP and starch in the diet. The NSP digestibility was calculated using 1 ) the $\mathrm{CHO}$ level in the diet; 2) the $\mathrm{CHO} \mathrm{ADC;} \mathrm{3)} \mathrm{the} \mathrm{level} \mathrm{of}$ NSP and starch in the diet; and 4) an assumption for the starch ADC of $99.5 \%$ for 
extruded diets and $91 \%$ for pelleted diets. Section 4 will elaborate on the decision to use these ADC values for starch. An overview of the calculations used and elucidation of the composition of the dataset is provided as supplementary information.

\section{Overview studies}

Applying the described criteria, data were collected from a total of 19 published (Table 1) and 6 unpublished studies. The unpublished studies were completed at the Aquaculture and Fisheries Group (Wageningen University, The Netherlands). The experiments included in the data set were mostly testing multiple diets. Combined, the 25 studies provided 100 different diets with their corresponding digestibility. Among these 100 different diets, two diets were fed at two different feeding levels and another two diets at three different feeding levels, resulting in 106 cases with known diet composition and ADC.

Table 1 gives an overview of the dietary nutrient composition, the nutrient digestibility and other related parameters ( $F C R$, initial bodyweight, etc.) of the included cases. As Table 1 shows, the carbohydrate digestibility is only reported for 29 of the 106 diets, with an average ADC of $71.5 \%$. The NSP digestibility is only presented by Leenhouwers et al. (2007a), Amirkolaie et al. (2006) and Haidar et al. (2016). These three studies show an average ADC of $26.4 \%$ for the NSP fraction, indicating the ability of tilapia to digest NSP to a certain extent. However, the sample size is small and the variation is large.

\section{Statistics}

Correlations between dietary NSP level, $\mathrm{CHO}$ and NSP digestibility, as well as and factors like nutrient composition of the diet, feeding level, FCR etc., were explored with Pearson's correlation coefficient. Linear regression equations were estimated when the Pearson's correlation coefficient was significant. The cases (106) were used as experimental units. A one-way ANOVA was used to compare means between: the production method (pelleting vs. extrusion) and starch digestibility; and reported vs calculated carbohydrate digestibility. All statistics were performed using the IBM Statistical Package for the Social Sciences (SPSS) program ( $v$ 23.0, New York, United States).

\section{Total carbohydrates}

Published data on total carbohydrate $(\mathrm{CHO})$ digestibility is limited. The constructed data set of the current study contained digestibility values for a total of 106 reported dietary treatments (diets and feeding level). The $\mathrm{CHO}$ digestibility was reported (averaging $71.5 \%$ ) form only 29 dietary treatments in the data set. For the remaining 77 treatments, the $\mathrm{CHO}$ digestibility was calculated from the known digestibility of other nutrients (for details on calculations see SI); being on average $64.9 \%$ and not different from the reported $\mathrm{CHO}$ digestibility $(P>0.1$; Figure 1$)$. Within the whole data set, there was a large variability in $\mathrm{CHO}$ digestibility, ranging 
Table 1 Reported average dietary nutrient composition, apparent digestibility coefficient $(A D C)$ and experimental parameters \pm SD of the included diets used for the meta-analysis.

\begin{tabular}{|c|c|c|}
\hline & Average & Number of diets \\
\hline \multicolumn{3}{|l|}{ Nutrient composition (g/ 100g DM) } \\
\hline Dry matter $(\mathrm{g} / \mathrm{kg})$ & $92.3 \pm 3.1$ & 97 \\
\hline Crude protein & $37.3 \pm 7.0$ & 100 \\
\hline Fat & $10.3 \pm 4.1$ & 100 \\
\hline 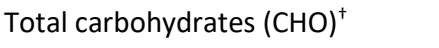 & $43.2 \pm 9.0$ & 100 \\
\hline Nitrogen free extract & $39.8 \pm 9.4$ & 35 \\
\hline Starch & $27.0 \pm 10.4$ & 24 \\
\hline Non starch-polysaccharides (NSP) & $19.6 \pm 15.5$ & 12 \\
\hline Crude fibre & $5.2 \pm 2.5$ & 43 \\
\hline Energy $(\mathrm{kJ} / \mathrm{g})$ & $20.2 \pm 1.0$ & 90 \\
\hline Ash & $9.2 \pm 2.5$ & 100 \\
\hline Calcium & $1.42 \pm 0.82$ & 18 \\
\hline Phosphorus & $1.06 \pm 0.37$ & 41 \\
\hline \multicolumn{3}{|l|}{$A D C(\%)$} \\
\hline Dry matter & $74.2 \pm 9.9$ & 86 \\
\hline Crude protein & $88.8 \pm 5.6$ & 106 \\
\hline Fat & $87.5 \pm 12.0$ & 106 \\
\hline Total carbohydrates & $71.5 \pm 15.9$ & 29 \\
\hline Nitrogen free extract & $73.3 \pm 13.6$ & 21 \\
\hline Starch & $96.2 \pm 4.4$ & 26 \\
\hline Non starch-polysaccharides & $26.4 \pm 26.8$ & 14 \\
\hline Energy & $80.8 \pm 9.2$ & 96 \\
\hline Ash & $48.9 \pm 10.5$ & 73 \\
\hline Calcium & $27.4 \pm 11.7$ & 14 \\
\hline Phosphorus & $64.1 \pm 25.6$ & 27 \\
\hline \multicolumn{3}{|l|}{ Experimental parameters } \\
\hline Feeding level $\left(\mathrm{g} / \mathrm{kg}^{0.8} / \mathrm{d}\right)$ & $13.0 \pm 4.3$ & 98 \\
\hline Initial body weight (g) & $72 \pm 94.9$ & 106 \\
\hline Feed Conversion ratio (FCR) & $1.34 \pm 0.46$ & 79 \\
\hline Specific growth rate (SGR, \%/d) & $1.70 \pm 0.69$ & 80 \\
\hline Length of trial (d) & $64 \pm 56$ & 100 \\
\hline \multicolumn{3}{|l|}{ Manufactured by; } \\
\hline Steam pelleting & - & $62^{\ddagger}$ \\
\hline Extrusion & - & $44 \S$ \\
\hline
\end{tabular}

${ }^{+}$Calculated on DM basis as: 100 - (crude protein + fat + ash).

" Köprücü and Özdemir, 2005; Amirkolaie, 2006; Leenhouwers et al., 2007a; El-Saidy and Gaber, 2003; Amirkolaie et al., 2006; Amirkolaie et al., 2005; El-Shafai et al., 2004; Obirikorang et al., 2015; Gaber, 2006; Gaber, 2005; Degani et al., 1997; Sintayehu et al., 1996; Schneider et al., 2004.

$\S$ Dong et al., 2010; Saravanan et al., 2012; Schrama et al., 2012; Haidar et al., 2016; Tran-Ngoc et al., 2016; Deng et al., 2016. 
from 12 to $95 \%$, with an average of $67 \%$. A CHO ADC of $12 \%$ was calculated for a diet high in protein and fat, including of $15 \%$ cellulose (Saravanan et al., 2012). Whereas a CHO ADC of $95 \%$ was found for a casein diet $(34.5 \%)$, with only wheat starch (50.5\%) as a carbohydrate source (Sintayehu et al., 1996).

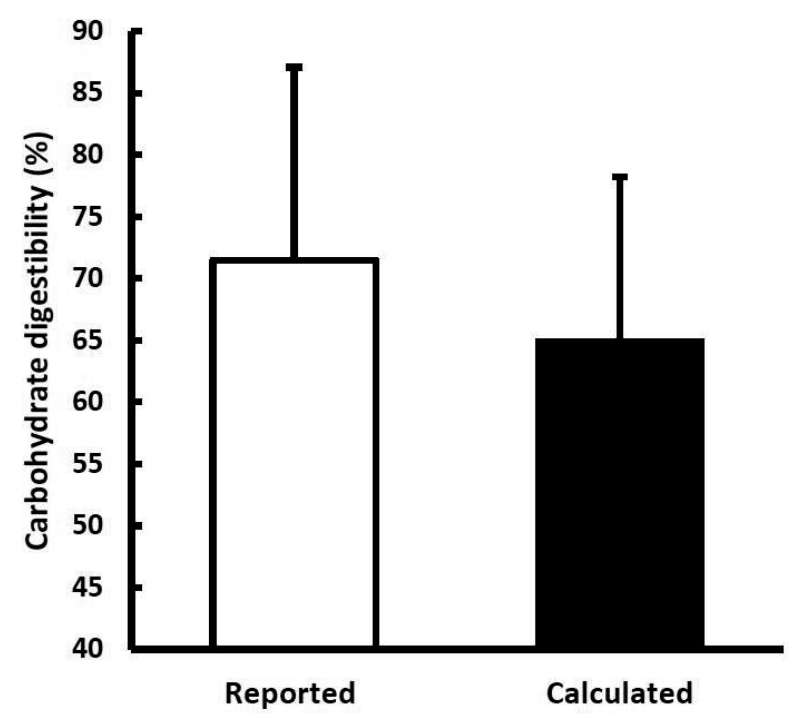

Figure 1 Comparison between the carbohydrate digestibility for the reported (29) and calculated (77) cases. Each bar shows overall mean with standard deviation represented with error bars.

Table 2 shows the correlation between the $\mathrm{CHO}$ digestibility and the experimental design, diet composition and ADC of other nutrients. Increasing mean initial body weight of the fish increases the $\mathrm{CHO} A D C(P<0.05)$. Information on the effect of fish weight on $\mathrm{CHO}$ ADC is scarce. A study by Pen-Hsing and Shi-Yen (1993) indicated that digestibility of starch was higher in tilapia (Oreochromis niloticus $\mathrm{x}$ $O$. aureus) with an higher initial weight of $4.55 \mathrm{~g}$, compared to fish with a lower initial weight of $0.46 \mathrm{~g}$. It is a long established fact that the gastrointestinal tract (GI-tract) of larvae and small fish is less developed and, shorter compared to adult fish (Govini, 1986). In addition, the production of digestive enzymes is lower for smaller fish, most likely related to the developmental stage of the digestive tract (Lauff and Hofer, 1984). The feeding level did not affect the CHO ADC, which is in line with some studies (Azevedo et al., 1998; Storebakken and Austreng, 1987) on rainbow trout (Oncorhynchus mykiss) and Atlantic salmon (Salmo salar L.). On the contrary, both Schrama et al. (2012) and Haidar et al. (2016) showed a reduction in ADC with increased feeding levels in Nile tilapia (Oreochromis niloticus), for all nutrients, including $\mathrm{CHO}$. In these two Nile tilapia studies, the feeding levels were approximately 1.5 and 3 times maintenance, and apparent satiation. With increasing $\mathrm{CHO}$ ADC the FCR improves $(P<0.01)$, because a larger part of the $\mathrm{CHO}$ is utilised for growth and metabolism. 
Table 2 Correlation between carbohydrate digestibility ( $Y$ variable; in \%) and parameters related to the design of the experimental design, to diet formulation and to apparent digestibility coefficients of other nutrients (ADC). Values represent the Pearson Correlation coefficient and the number of observations.

\begin{tabular}{|c|c|c|c|}
\hline$x$ & $\begin{array}{l}\text { Correlation } \\
\text { coeffcient }\end{array}$ & $\begin{array}{l}\text { Number of } \\
\text { observations }\end{array}$ & Estimated equation $\mathrm{CHO} A D C(\mathrm{Y})$ \\
\hline \multicolumn{4}{|l|}{ Experimental design } \\
\hline Body weight start (g) & $0.219^{*}$ & 106 & $Y=64(S E 1.7)+0.033($ SE 0.01) $\times X$ \\
\hline Feed intake (g DM/ $\left./ \mathrm{kg}^{0.8} / \mathrm{d}\right)$ & ns & 98 & $\mathrm{n} / \mathrm{s}$ \\
\hline FCR & $-0.446 * *$ & 79 & $Y=86$ (SE 4.7) - 14.5 (SE 3.3) $x X$ \\
\hline \multicolumn{4}{|l|}{ Dietary level (\%) } \\
\hline Crude protein & ns & 106 & $\mathrm{n} / \mathrm{s}$ \\
\hline Fat & $-0.375 * *$ & 106 & $Y=80$ (SE 3.5) - 1.32 (SE 0.32) $\times X$ \\
\hline Energy & $-0.207^{*}$ & 96 & $Y=125$ (SE 29) - 2.9 (SE 1.4) $\times X$ \\
\hline Total carbohydrates & $0.222 * *$ & 106 & $Y=51($ SE 6.7) $+0.4($ SE 0.15) $\times X$ \\
\hline Starch & $0.740 * *$ & 34 & $Y=44($ SE 4.9) + 1.1 (SE 0.17) $\times X$ \\
\hline Crude fibre & $-0.748 * *$ & 43 & $Y=85$ (SE 3.5) - 4.4 (SE 0.61) $\times X$ \\
\hline \multicolumn{4}{|l|}{$A D C(\%)$} \\
\hline Crude protein & $0.462 * *$ & 106 & $Y=-39($ SE 20) + 1.19 (SE 0.22) $\times X$ \\
\hline Fat & $0.630 * *$ & 106 & $Y=-0.2(S E$ 8.2) + 0.8 (SE 0.09) $\times X$ \\
\hline Energy & $0.791^{* *}$ & 96 & $Y=-34($ SE 8.2) + 1.3 (SE 0.10) $\times X$ \\
\hline Starch & $0.525^{* *}$ & 26 & $Y=69($ SE 8.5) + $0.06($ SE 0.09) $\times X$ \\
\hline Ash & ns & 73 & $\mathrm{n} / \mathrm{s}$ \\
\hline
\end{tabular}

Notes. CHO, total carbohydrates; ns, not significant; $*, P<0.05 ; * *, P<0.01 ; \mathrm{n} / \mathrm{s}$, not shown, because being not significant.

The crude protein level in the diet does not influence the CHO ADC $(P>0.05)$. The fat level and energy level in the diet are highly correlated as fat has a high energy equivalent $(39.5 \mathrm{~kJ} / \mathrm{g})$, increasing the total energy content of the diet. Appropriate levels of carbohydrates should be incorporated in the diets to improve the energy availability, sparing the catabolism of protein and lipids for energy (Wilson, 1994). With increasing energy levels in the diet and thereby thus most likely increasing fat levels, the role of carbohydrates in providing energy may become less important as sufficient fat, and thus energy, is provided. However, the $\mathrm{CHO}$ composition in the diet in particular has a large effect on the $\mathrm{CHO}$ ADC, in as much as the carbohydrate fraction consists of highly digestible starch and sugars and poorly digestible NSP. As expected, increasing levels of starch positively correlates $(P<0.01)$ with the $\mathrm{CHO} A D C$, as the fraction of highly digestible starch is likely to go up with increasing starch levels (Figure $2 a$ ). With every $1 \%$ of extra starch in the diet, the CHO ADC increases at almost the same rate $(1.05 \%)$. The level of crude fibre in the diets has a much larger effect on the CHO ADC compared to the starch level in the diet (Figure $2 \mathrm{~b}$ ). With each $1 \%$ increase in dietary crude fibre the $\mathrm{CHO}$ ADC decreases by $4.4 \%$. Crude fibre analyses give a rough indication of the cellulose and lignin content of the diet rather than the total NSP fraction. The diets for which the crude fibre fraction was given had, on average (43 cases), 4.8 
times more NSP than the reported level of crude fibre. This high increase in poorly digestible NSP with increasing levels of dietary crude fibre, explains the large impact crude fibre has on the CHO ADC.
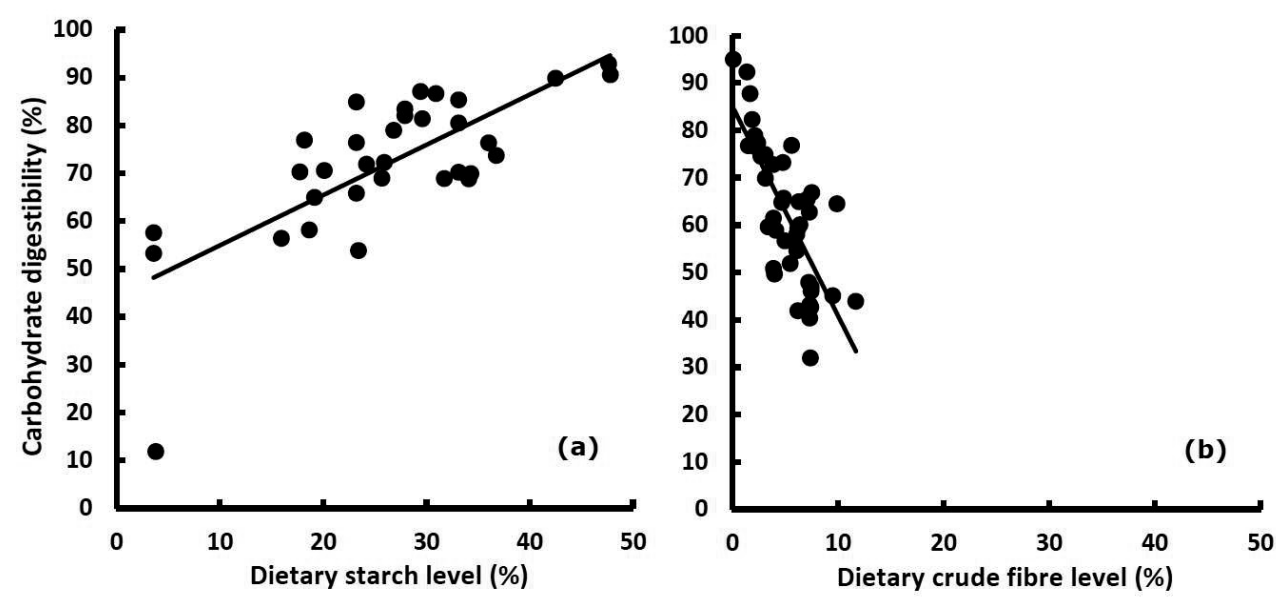

Figure 2 Relationship between the dietary starch level and (a) dietary crude fibre level, and (b) with the carbohydrate apparent digestibility coefficient (\%) for tilapia.

The starch ADC is directly related with an improvement in the $\mathrm{CHO}$ ADC, as starch in general takes up the largest part of the carbohydrate fraction in the diet. With an increase in $\mathrm{CHO} A D C$, more energy is digested in the form of $\mathrm{CHO}$, thus increasing the energy $\mathrm{ADC}(P<0.01)$. The overall trend when looking at the correlation between the $\mathrm{CHO} A D C$ and the ADC of the other nutrients (except ash) is that digestibility is always highly positively correlated with increasing CHO ADC. This trend, especially the correlation with protein, fat and energy, may be better explained by the quality of the diet (ingredients, formulation, processing) of the diet, rather than the $\mathrm{CHO}$ ADC actually having an effect on protein, fat and energy digestibility. The quality of the diet (i.e. choice of ingredients) and processing method (i.e. extrusion vs. steam pelleting) are factors known to influence the overall nutrient ADC, irrespective of the nutrient composition (Alonso et al., 2000; Cheng and Hardy, 2003). In addition, high quality diets are generally low in crude fibre and NSP. Therefore, the known negative effects of NSP on the digestibility of other nutrients (including $\mathrm{CHO}$ ) are likely to be further aggravated with increasing levels of dietary crude fibre and NSP. Examples of how NSP can negatively affect nutrient digestibility are: dietary viscosity, which can hamper the mixing of enzymes within the chyme and the absorption of nutrients, due to hindered effective interaction at the mucosal surface (Choct, 1997); increased faecal losses of bile acids (which are important for fat digestion), as a consequence of binding to NSP and/or an increased deconjugation of bile acids by microbial activity, stimulated by NSP (Sinha et al., 2011). The quality of the diet, and thus the digestibility of the nutrients (fat and protein), is likely to contribute to not only an improvement in the $\mathrm{CHO}$ ADC $(P<0.01)$, but also in the FCR. 


\section{Starch}

As seen in section 3 on total carbohydrates, the starch fraction is important for the $\mathrm{CHO} A D C$, as starch is highly digestible in tilapia. Starches (corn, wheat, etc.) are often included to limit the amount of protein utilised for energy (Wilson, 1994). For example, Wang et al. (2005) showed an improvement in the protein efficiency ratio in Nile tilapia when including $22-46 \%$ of starch in the diet, compared to levels of $6 \%$ or $14 \%$. From the 26 diets reporting the ADC of starch, 10 diets were manufactured by steam pelleting with an average ADC of $91.0 \pm 2.25 \%$, while the other 16 diets were manufactured by extrusion and had an ADC of $99.4 \pm 0.7 \%$ (Figure 3). These differences in starch ADC are in line with results from Amirkolaie et al. (2006), where starch gelatinised by extrusion showed an ADC of around $99.3 \%$, while native starch had an ADC of around $93.8 \%$. Similar results are shown for rainbow trout, where gelatinising native starch increased the ADC of starch from $38.1 \%$ to $86.5 \%$ (Bergot and Breque, 1983). Cooking carbohydrates, to enhance their digestibility through gelatinisation, dates back to the 1940 's for trout (Phillips Jr and Brockway, 1956).

\section{Non starch polysaccharides}

\section{Non-starch polysaccharides in fish feed}

Nowadays plant proteins are increasingly frequently used more used to replace fishmeal in fish feeds, in order to reduce costs and keep pace with the increasing demand for high quality protein. This has resulted in a steady decline in dietary fish meal (and fish-oil) in aquafeeds (Carter and Hauler, 2000; Shepherd and Jackson, 2013). A major downside of using plant ingredients such as soya, wheat, rye and legume seeds is the presence of a variety of anti-nutritional substances (ANS), often limiting the inclusion of these ingredients in aquafeeds. NSP is one of the major ANS present in plant ingredients (Choct, 1997; Francis et al., 2001; Sinha et al., 2011). Multiple studies in broilers and pigs have shown that feedstuffs containing NSP negatively affect nutrient digestibility and thus limit growth (Choct and Annison, 1992; Annison, 1993; Knudsen et al., 1993; Jørgensen et al., 1996; Mroz et al., 2000). Similar studies in fish are scarce.

NSP also influences chyme viscosity. Dietary soluble NSP increases the viscosity, thereby decreasing the passage rate and diffusion rates of digestive enzymes and substrates, whereas insoluble NSP increases the passage rate. NSP may influence the gut morphology, physiology and mucus layer, affecting the endogenous secretion of water, proteins, electrolytes and lipids. These changes can lead to reduced nutrient digestibility (Choct, 1997; Choct and Kocher, 2000; Sinha et al., 2011). In pigs for example, inclusion of cellulose in the diet resulted in the shortening of villi length. Shortening of villi results in a loss of intestinal surface area. As a result, absorption of nutrients is decreased (Sinha et al., 2011). A lower passage rate, as a result of increased levels of soluble NSP in the diet, may decrease the oxygen tension in the gut, favouring anaerobic microbiota. An 
increase in anaerobic microbiota can enhance the production of toxins and the deconjugation of bile salts, which are important for fat digestion (Carey et al., 1983; Choct, 1997; Sinha et al., 2011). However, information about the effect of NSP on the metabolism in fish is scarce.

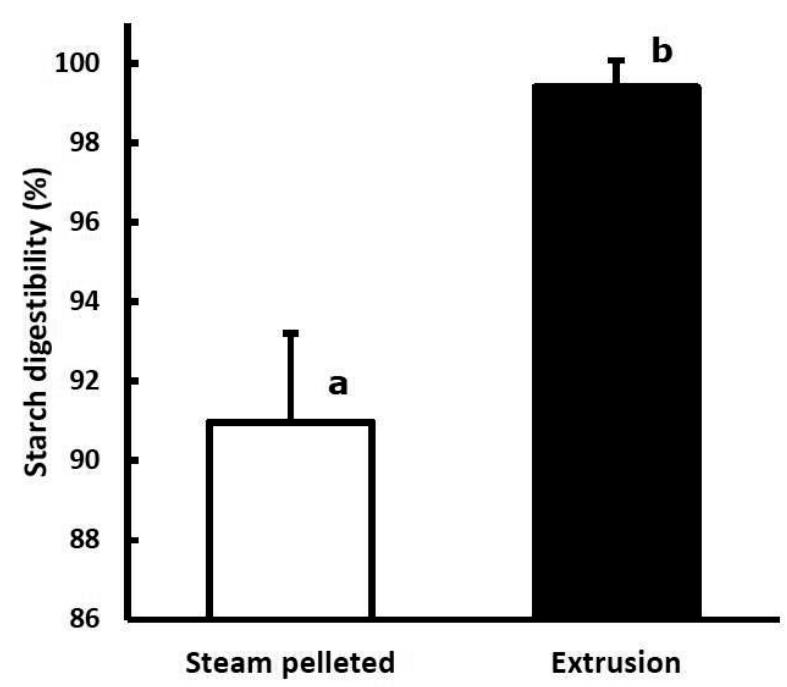

Figure 3 Effect of pellet manufacturing (steam pelleting vs. extrusion) on the starch digestibility in tilapia. Each bar shows overall mean with standard deviation represented with error bars. Bars with different lower case letters are significantly different $(P<0.05)$.

The calculated dietary NSP level in the present meta-analyses averaged $17.5 \% \pm$ 8.6 , with a minimum of $1.4 \%$ and a maximum of $37.9 \%$. This large variation in dietary NSP level is simply due to the choice of ingredients, i.e., using soybean meal as a protein source versus an isolate or concentrate, like soy protein isolate or pea protein concentrate. In line with the above theory, increasing the levels of dietary NSP was shows to negatively correlate with the digestibility of crude protein, fat and energy $(P<0.001$; Table 3.). Figure $4 a$ shows the relationship between the dietary NSP level and energy digestibility; for each percent increase in dietary NSP, the energy ADC decreases by $0.7 \%$. Similarly, as in figure 4 , Haidar et al. (2016) showed that increasing the dietary NSP level causes a decrease in both the energy digestibility and the protein digestibility $(P<0.05)$. In line with a lower availability of nutrients, due to a decrease in nutrient digestibility, the FCR increases by 0.03 for every percent increase in dietary NSP (Figure 4b). 
Table 3 Correlation between the total non-starch polysaccharide (NSP) level in the diet (X variable in the equation, in \%) and fish performance parameters and apparent digestibility coefficients (ADC) of other nutrients. Values represent the Pearson Correlation coefficient and the number of observations.

\begin{tabular}{|c|c|c|c|}
\hline Y & $\begin{array}{l}\text { Correlation } \\
\text { coefficient }\end{array}$ & $\begin{array}{l}\text { Number of } \\
\text { observations }\end{array}$ & Estimated equation $(Y)$ using NSP level $(X)$ \\
\hline \multicolumn{4}{|l|}{ Performance } \\
\hline FCR & $0.500 * *$ & 79 & $Y=0.9($ SE 0.10$)+0.03($ SE 0.006) $\times X$ \\
\hline SGR $(\% / d)$ & ns & 80 & $\mathrm{n} / \mathrm{s}$ \\
\hline \multicolumn{4}{|l|}{$A D C(\%)$} \\
\hline Crude protein & $-0.491 * *$ & 105 & $Y=100($ SE 2.3) $-0.7($ SE 0.12) $\times X$ \\
\hline Fat & $-0.500 * *$ & 105 & $Y=95$ (SE 1.1) - $0.3($ SE 0.06) $\times X$ \\
\hline Energy & $-0.662 * *$ & 95 & $Y=93($ SE 1.6) $-0.7($ SE 0.08) $\times X$ \\
\hline Starch & ns & 26 & $\mathrm{n} / \mathrm{s}$ \\
\hline Ash & ns & 72 & $\mathrm{n} / \mathrm{s}$ \\
\hline
\end{tabular}

Notes. ns, not significant; $* *, P<0.01 ; \mathrm{n} / \mathrm{s}$, not shown, because being not significant.

\section{Non-starch polysaccharide digestibility}

Digestive enzymes, such as a-amylase, disaccharidase and a-glucosidase, in combination or independently, are able to hydrolyse a-glycoside bonds (Stone, 2003). NSP however, generally remains undigested in monogastric animals. The enzymes needed to hydrolize the B-glycosidic bonds of the long NSP chains such as cellulase, B-xylanases, B-glucanases and $B$-galactases, are scarce or nonexisting in the GI-tract of fish (Kuz'mina, 1996; Choct, 1997; Stone, 2003; Sinha et al., 2011). Cellulase activity has been reported in several carp species, e.g. bighead carp (Aristichthys nobilis), grass carp (Ctenopharyngodon idella) and common carp (Cyprinus carpio) (Chakrabarti et al., 1995; Li et al., 2009; Banerjee et al., 2016). In tilapia this information is rare, however a study by Saha et al. (2006) measured cellulase activity from isolated bacterial strains from the gut of tilapia (Oreochromis mossambica), measuring a maximum cellulase activity of 67 $\mathrm{U} / \mathrm{ml}$. In Banerjee et al. (2016), besides cellulase, xylanase activity and xylanase producing microbial symbionts were detected in the proximal and distal intestine of six freshwater carp species. The assumption was that the xylanase and cellulase producing microbiota were autochthonous as the fish were starved for $48 \mathrm{~h}$ prior to taking the GI-tracts, after which the GI-tracts were cleansed with sterilized $(0.9 \%)$ saline. German and Bittong (2009), suggest that fish ingest cellulase- and xylanase- producing microbes while feeding on detritus derived from plant matter, rather than these enzymes being produced by the autochthonous microbiota. Whether the cellulase and xylanase producing microbiota are endogenous, and/or whether the gut is colonised by ingested microbes while feeding on detritus, is unknown. Cellulase and xylanase are responsible for the breakdown of cellulose and xylans, respectively, which are the major NSP in plant matter (Sinha et al., 2011; Banerjee et al., 2016). Although present in the gut, the activity of cellulase 
is generally low ( $<70 \mathrm{U} / \mathrm{ml}$ ) (Chakrabarti et al., 1995; Banerjee et al., 2016). Besides that, the hydrolysis of cellulose is generally slow and incomplete and therefore possibly only occurs on a small scale in the gut (Schwarz, 2001).
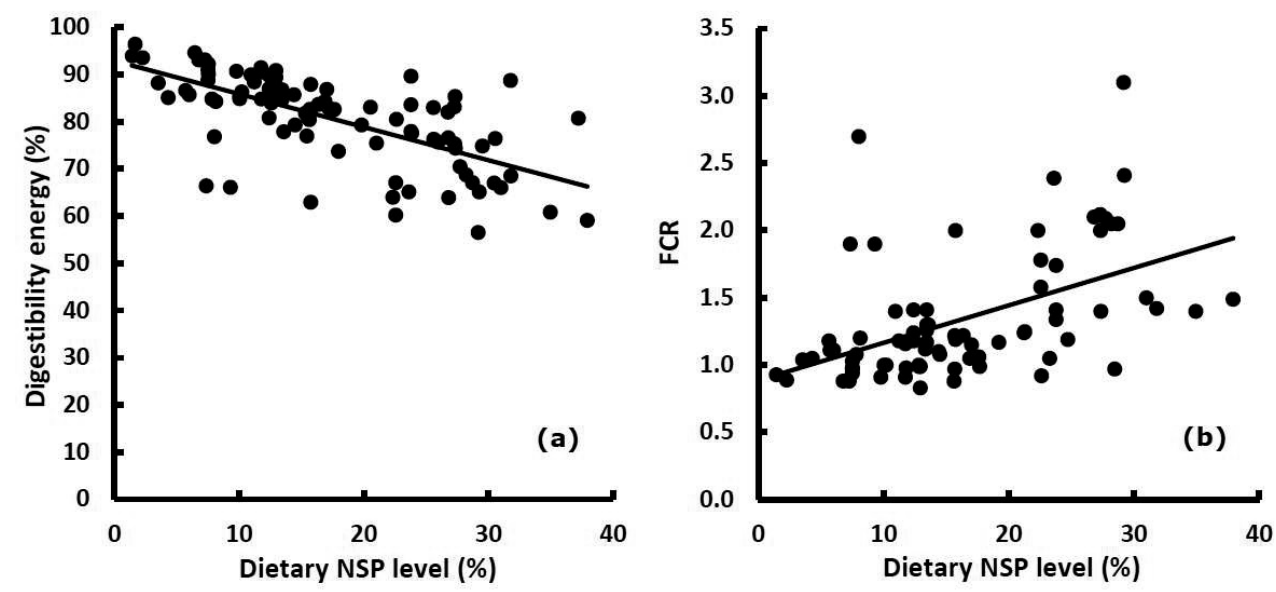

Figure 4 Relationship between dietary non-starch polysaccharide (NSP) level and the (a) energy digestibility, and (b) feed conversion ratio (FCR) for tilapia.

Although long considered as indigestible in fish, a few studies now show that part of the NSP fraction can be digested by tilapia (Amirkolaie et al., 2005; Leenhouwers et al., 2007a; Haidar et al., 2016). The wide range of NSP used in these studies differed in characteristics and properties, including endogenous digestibility. Amirkolaie et al. (2005) showed significant differences in the NSP digestibility when comparing guar gum (oligosaccharides) and cellulose, indicating that cellulose, with an ADC of $2.8 \%$, is more inert than guar gum, which has an ADC of $20.8 \%$. Similar results were found by Leenhouwers et al. (2007a), where replacement of $40 \%$ of a reference diet with either maize, wheat, barley or rye, resulted in significant differences in NSP digestibility. Solubility of the NSP is an important factor affecting the digestibility of the NSP fraction (Sinha et al., 2011). Leenhouwers et al. (2007a) made a distinction in NSP digestibility based on its solubility (soluble vs. insoluble), indicating negative values for the digestibility of the insoluble NSP fraction, independent of the NSP source (maize, wheat, barley and rye), with an ADC of up to $60 \%$ for the soluble NSP fraction. This is in line with the poor digestibility found for cellulose (insoluble), compared to guar gum (soluble), in Amirkolaie et al. (2005). In the present meta-analysis, the feeding level ( $\mathrm{g} \mathrm{DM} / \mathrm{kg}^{0.8} / \mathrm{d}$ ) had no effect on the NSP digestibility. On the contrary, Haidar et al. (2016) showed that with increasing feeding levels, feeding the same diet, the ADC of NSP decreased drastically. This suggests a threshold in the amount of NSP that can be digested.

From the present meta-analysis, an average NSP digestibility of $24.3 \%$ was found from the 95 cases presented. From these 95 cases, in only 11 cases was there a 
negative $A D C$, indicating that no NSP was digested. This indicates that in 83 of these cases NSP digestibility can be assumed, of which 12 had an ADC above $50 \%$. In three studies (Amirkolaie et al., 2005; Leenhouwers et al., 2007a; Haidar et al., 2016), where NSP ADC was reported, the NSP ADC averaged $26.4 \%$ for a total of 14 cases, which is highly comparable to the NSP ADC of $24.3 \%$ found by meta analyses. In cases where NSP ADC reported a negative value, if this value was hypothetically increased to $0 \%$, then the ADC value would increase to $28.4 \%$. In other monogastric animals, like pigs and poultry, it is well established that fermentability varies considerably among different types of NSP, with, for instance, lignin being very resistant and pectins usually undergoing complete fermentation (Williams et al., 2001; Williams et al., 2017). In tilapia this also seems to be the case based on the limited amount of literature on NSP ADC. For example, in a study by Maas et al. (2019), different experimental diets contained contrasting types of NSP, by incorporating wheat bran, sunflower meal or citrus pulp to a reference diet. The ingredients were chosen for their contrast in NSP composition; wheat bran being relatively rich in hemicellulose, sunflower meal rich in cellulose and citrus pulp rich in pectins. The pectin-rich citrus pulp diet showed the highest NSP digestibility at $31.7 \%$, followed by the sunflower $(18.8 \%)$ and wheat bran (17.1\%) diets.

Comparable to the digestibility of the $\mathrm{CHO}$ fraction, the digestibility of the NSP fraction increases with an increase in initial body weight $(P<0.001$; Table 4.). The digestion of NSP in fish occurs through fermentation in the gut (Bergman, 1990; Metzler-Zebeli et al., 2010). The GI-tract of larvae and adult fish differs largely, with a more complex morphology and histology for adult fish (Govoni et al., 1986). As the gut is the site of action of gut fermentation, a more developed gut in bigger fish could explain for the higher NSP digestibility with increasing fish size. Increasing NSP digestibility significantly $(P<0.05)$ contributes to an improvement in FCR, as seen by the negative correlation coefficient. The digestibility of NSP decreases with increasing levels of crude fibre $(P<0.05)$. Crude fibre analyses give a rough indication of the cellulose and lignin content of the diet rather than the total NSP fraction. Amirkolaie et al. (2005) shows that cellulose is almost completely inert to digestion by tilapia. Increasing levels of almost inert dietary crude fibre may therefore decrease the total NSP ADC. The energy and fat digestibility increased with increasing NSP digestibility $(P<0.01)$ in tilapia. With an increase in NSP digestibility, the NSP level in the gut is likely to go down and with it the potential negative effects NSP has on the GI-tract and nutrient utilisation. A NSP ADC of $24.3 \%$ (average found in present study), would result in an increase of the energy ADC by $3 \%$ compared to having no NSP digestibility in tilapia. 
Table 4 Correlation between non-starch polysaccharides (NSP) digestibility (Y variable; in $\%)$ and parameters related to the design of the experiment, to diet formulation and to apparent digestibility coefficients of other nutrients (ADC). Values represent the Pearson Correlation Coefficient and the number of observations.

\begin{tabular}{|c|c|c|c|}
\hline$x$ & $\begin{array}{l}\text { Correlation } \\
\text { coeffcient }\end{array}$ & $\begin{array}{l}\text { Number of } \\
\text { observations }\end{array}$ & Estimated equation NSP ADC $(\mathrm{Y})$ \\
\hline \multicolumn{4}{|l|}{ Experimental design } \\
\hline Body weight start (g) & $0.967 * *$ & 95 & $\mathrm{Y}=17(\mathrm{SE} 3.8)+0.1(\mathrm{SE} 0.03) \times X$ \\
\hline Feed intake (g DM kg ${ }^{-0.8} \mathrm{~d}^{-1}$ ) & ns & 87 & $\mathrm{n} / \mathrm{s}$ \\
\hline FCR $(\mathrm{g} / \mathrm{g})$ & $-0.245^{*}$ & 70 & $Y=43($ SE 11) -17 (SE 7.6) $\times X$ \\
\hline \multicolumn{4}{|l|}{ Dietary level (\%) } \\
\hline Crude protein & ns & 95 & $\mathrm{n} / \mathrm{s}$ \\
\hline Fat & $-0.287 * *$ & 95 & $Y=45$ (SE 8.0) - 2.1 (SE 0.74) $\times X$ \\
\hline Energy & ns & 85 & $\mathrm{n} / \mathrm{s}$ \\
\hline Total carbohydrates & $0.213^{*}$ & 95 & $Y=-8($ SE 15) +0.7 (SE 0.35) $\times X$ \\
\hline Starch & ns & 27 & $n / s$ \\
\hline Crude fibre & $-0.379 *$ & 41 & $Y=57$ (SE) - 7 (SE 2.6) $x X$ \\
\hline \multicolumn{4}{|l|}{$A D C(\%)$} \\
\hline Crude protein & ns & 95 & $\mathrm{n} / \mathrm{s}$ \\
\hline Fat & $0.319 * *$ & 95 & $Y=-46($ SE 22) $+0.8($ SE 0.25) $\times X$ \\
\hline Energy & $0.412^{* *}$ & 86 & $Y=-92($ SE 28) + 1.4 (SE 0.34) $\times X$ \\
\hline Starch & ns & 29 & $\mathrm{n} / \mathrm{s}$ \\
\hline Ash & $0.395^{* *}$ & 67 & $Y=-9($ SE 10) $+0.7($ SE 0.21) $\times X$ \\
\hline
\end{tabular}

Notes. ns, not significant; $*, P<0.05 ; * *, P<0.01 ; \mathrm{n} / \mathrm{s}$, not shown, because being not significant.

\section{Fermentation and volatile fatty acids}

The digestion of NSP in fish, as shown in the present meta-analysis and in studies by Amirkolaie et al. (2005), Haidar et al. (2016) and Leenhouwers et al. (2007a) using tilapia, occurs through fermentation in the gut. Other than starch and sugars, which are generally directly digested in the stomach, NSP are mainly fermented in the gut. During the fermentation of NSP and carbohydrates, short chain fatty acids (SCFA) and the gases $\mathrm{H}_{2}, \mathrm{CO}_{2}$ and $\mathrm{CH}_{4}$ are formed through microbial anaerobic glycolysis (Bergman, 1990; Metzler-Zebeli et al., 2010). The SCFA are often referred to as volatile fatty acids (VFA). With fermentation of carbohydrates, $73 \%$ of the $\mathrm{C}$ fraction can be converted into completely metabolisable VFA, whereas the remainder is lost $\left(\mathrm{CO}_{2}\right)$ (Williams et al., 2001). The VFA produced can rapidly be absorbed by the colonic lumen. In monogastric animals, around $95-99 \%$ of the VFAs produced are absorbed before reaching the rectum (Von Engelhardt et al., 1989; Scheppach, 1994). The principle end products of VFA from carbohydrate fermentation are acetate, propionate and butyrate and to a lesser extent formate, valerate, caproate and the branched chain acids isobutyrate and isovalerate (Williams et al., 2001; Macfarlane and Macfarlane, 2003). Levels of the intermediate lactic acid, which is commonly found in monogastrics like pigs 
(Argenzio and Southworth, 1975), have not been reported in fish, but most likely, they have not been measured. However, with the use of an in vitro gas production technique, using freshly collected intestinal content from Nile tilapia, lactic acid was measured (Leenhouwers et al., 2008). Hereby, four different substrates were used (glucose, wheat starch, arabinoxylan, whole wheat), the substrate used strongly influenced the amount of lactic acid produced $(P<0.001) .11 .06 \mathrm{mM}(\mathrm{g} /$ organic matter weighed in) of lactic acid was measured for glucose and $2.82 \mathrm{mM}$ for wheat starch, while there was almost no lactic acid found for the substrates arabinoxylan and whole wheat $(<0.04 \mathrm{mM})$. The total VFA (acetic + propionic + butyric + iso-butyric + valeric + iso-valeric acid) level measured for the glucose amounted to $3.01 \mathrm{mM}$, which is 3.7 times lower compared to the amount of lactic acid $(11.06 \mathrm{mM})$. The in vitro study of Leenhouwers et al. (2008) makes the assumption plausible that lactic acid can be produced in Nile tilapia. It should be noted that both glucose and wheat starch are not commonly incorporated in aqua feeds, of the 25 studies included in the present analyses only one used wheat starch as an ingredient (Sintayehu et al., 1996). If other starches, like maize starch, result in similar levels of lactic acid, as compared to wheat starch, is currently unknown.

The fermentation in fish takes place throughout the gut with increasing microbial activity from the proximal to the distal the end of the hindgut, conjointly with increasing levels of VFA towards the proximal end (Mountfort et al., 2002; Amirkolaie et al., 2006; Leenhouwers et al., 2007a). This is comparable with pigs, where easily accessible compounds are utilised in the upper part of the GI-tract, whereas complex low soluble substrates are fermented and utilised in the lower GI-tract sections (Knudsen et al., 2012). In general, the composition of the VFA produced remains within a range of $60-75 \%$ acetate, $15-25 \%$ propionate and $10-$ $15 \%$ butyrate for monogastric animals fed conventional diets (Bugaut, 1987). However, in humans the rate of breakdown of individual NSP and the ratio of fermentation products (acetate, butyrate and propionate) vary depending on the type of polysaccharide present (Macfarlane and Macfarlane, 2003). Similar characteristics have been seen in cows, where the amount of VFA and the ratio between acetate, butyrate and propionate differed depending on the cereal used, when comparing hay ( $100 \%)$ versus a concentrate of cracked corn ( $81 \%)$, soybean ( $9 \%$ ) and timothy hay (10\%) (Russell 1998). Hitherto, little is known about the ratio of the VFA produced through fermentation in fish compared to pigs and poultry. Comparable with other monogastrics, Leenhouwers et al. (2007a) and Amirkolaie et al. (2006) found high levels of acetate ( $\pm 16.4 \mathrm{mM}$ on fresh digesta) and minor levels of propionate $( \pm 0.4 \mathrm{mM})$ and butyrate $( \pm 0.2 \mathrm{mM})$ in the distal gut of tilapia. An overview of studies measuring VFA in the distal gut of different fish species is given in table 5 , for species with predominately herbivorous feeding 


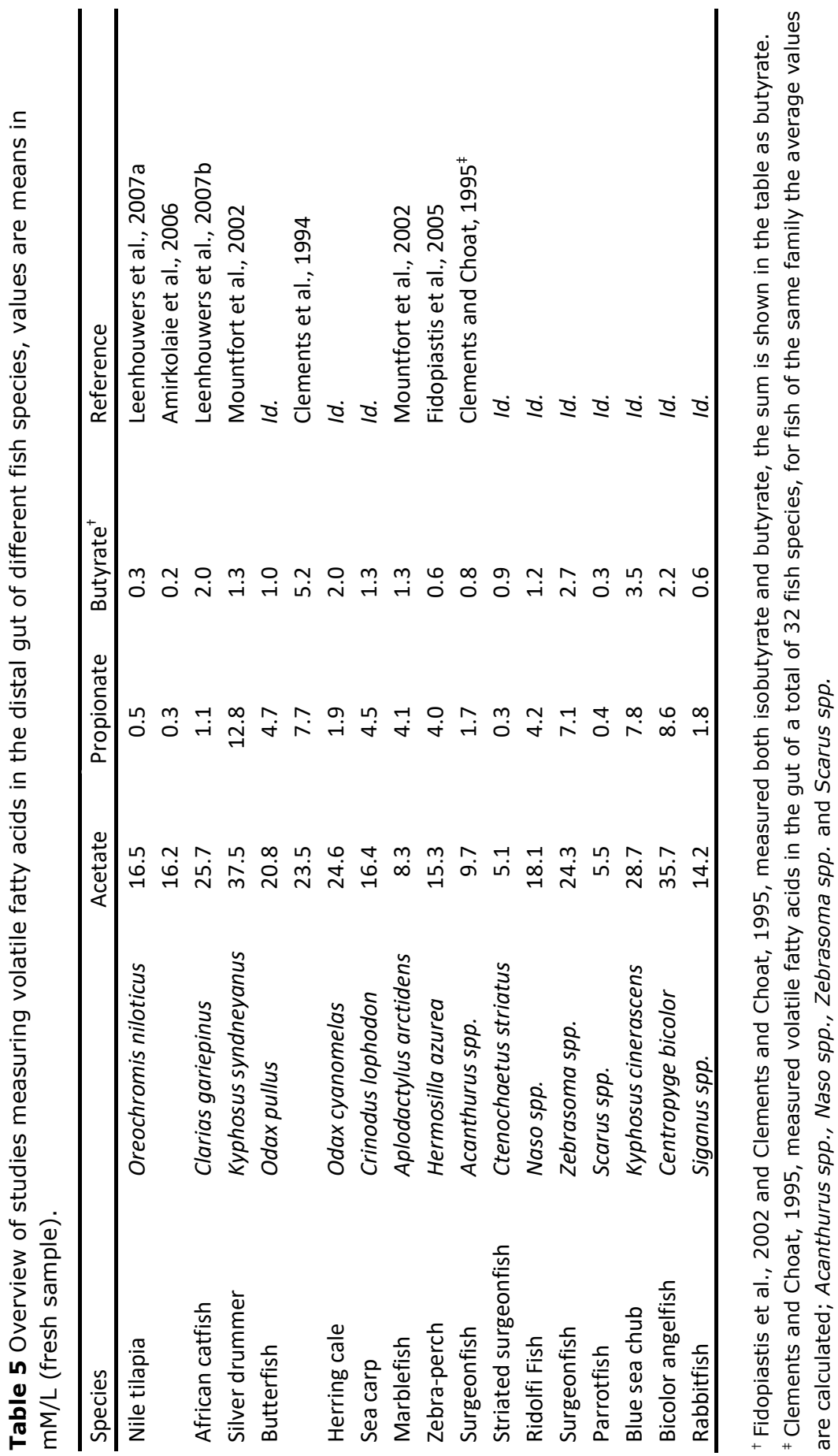


habits. Most of these studies measured VFA levels in different gut segments, showing increasing total VFA levels towards the proximal end (Leenhouwers et al., 2007a; Amirkolaie et al., 2005; Mountfort et al., 2002; Clements et al., 1994; Fidopiastis et al., 2006; Clements and Choat, 1995). The VFA composition is comparable among studies with proximate levels of $60-95 \%$ acetate, $5-25 \%$ propionate and $1-15 \%$ butyrate.

In an in vitro experiment, using the contents of the distal gut from common carp (Cyprinus carpio L), cumulative and composition differences in VFA products were found when the contents were inoculated with different substrates (oligosaccharides) (Kihara and Sakata, 2002). With NSP fermentation, the concentration, and thus the anti-nutritional effects of the NSP fraction, will be lowered. With NSP fermentation, the digestibility of the NSP and thus the carbohydrate fraction will improve, resulting in lower faecal waste and improved feed efficiency (Sinha et al., 2011). Besides these practical implications, the microbiota, intestinal- and animal health can be influenced by the production of VFA. The amount and composition of the fermentation end products will influence the animal, microbiota and intestine in different ways as they differ in characteristics and properties (Bergman, 1990; Claus et al., 2007). Acetate and propionate enter the blood passively due to a concentration gradient and are transported to the liver (Williams et al., 2001; Montagne et al., 2003). Acetate is used as energy substrate for muscle tissue via acetyl-coenzyme A syntheses (Clements et al., 1994). In the liver, propionate is used for gluconeogenesis, turning propionate into glucose, which provides an important source of energy for metabolic activities in the colon. Butyrate is primarily used as a direct source of energy by the colonocytes, providing energy for its metabolic activities and stimulating epithelial cell proliferation. This is mainly known for pigs and poultry. In fish, such data is lacking. By supplying energy in different ways, the contribution of VFA to the energy in the gut and body can be substantial (Von Engelhardt et al., 1989; Williams et al., 2001; Montagne et al., 2003). VFA was shown to inhibit growth of some bacterial pathogens in the gut of rabbits and pigs when in an acidic environment, similar effects on pathogens induced by VFA production in fish may occur as well (May et al., 1994; Montagne et al., 2003).

\section{NSP as functional feed ingredients}

NSP, including $\beta$-glucans, mannan-oligosaccharides (MOS), galactooligosaccharides (GOS) and fructo-oligosaccharides (FOS) (Nawaz et al., 2018), but also pectins (Wiese, 2019), may contribute as prebiotics to animal health by preventing pathogen adhesion, stimulating immune maturation and gut barrier function, and serving as fermentable substrates for gut bacteria. The increased production of intermediate metabolites, such as SCFA, can assist in balancing the immune system. In aquaculture, the NSP most frequently used as a prebiotic is probably $\beta$-glucans, their effects are often evaluated as modulations of innate immune responses by measuring gene expression or enhanced phagocytosis, reactive oxygen species, lysozyme activity and, more recently, trained immunity 
(Petit and Wiegertjes, 2016). These prebiotic products are added to fish feed, usually at low inclusion rates of $<1 \%$ as functional feed ingredients (Dalmo and Bøgwald, 2008). It should be noted that the $\beta$-glucans used as prebiotics generally originate from yeast, which has a different structure than the $\beta$-glucans from cereals and legumes. To which extent $\beta$-glucans from cereals and legumes have the same mode of action as those from yeast is unclear.

NSP may be increasingly added to fish feed as modulators of fish health but, for most if not all NSP, it remains undecided whether their modulatory effects are direct or gut microbiota mediated. In a preliminary study on the normal gut microbiota of common carp we could detect an abundant presence of Bacteroides bacteria, well known for their capacity to degrade and ferment carbohydrates, in line with earlier studies of cellulase activity (Chakrabarti et al., 1995; Li et al., 2009; Banerjee et al., 2016). In vitro fermentation of a commercially-available $\beta$ glucan functional feed ingredient indicated an increased presence of propionate, a SCFA with immunomodulatory properties, whose presence possibly helps explain earlier-noted immunomodulatory effects, such as inhibition of the expression of several pro-inflammatory genes (Falco et al., 2014).

Direct immune-modulatory effects of NSP could be achieved via recognition of, for example, $\beta$-glucans, by receptors present locally on cells in the gut. Yet, despite the frequent application of $\beta$-glucans in aquaculture practice, the exact receptors and downstream signalling remain to be described. In fish genomes, no clear homologue of Dectin-1 could be identified, a member of the C-type lectin receptor (CLR) family and the best-described receptor for $\beta$-glucans in mammals. Still, a recent transcriptome analysis of genes expressed in common carp macrophages, stimulated with $\beta$-glucan, highlighted differential regulation of a signalling pathway typical of CLR activation. Subsequent genome analysis identified a large number of candidate $\beta$-glucan receptor genes encoding for proteins, with at least one Ctype Lectin Domain (CTLD) typical of the CLR family. This large number could be narrowed down to a few genes with a typical sugar-binding motif in their CTLD, but these were not expressed in macrophages, the innate immune cell types often associated with recognition of $\beta$-glucans (Petit et al., 2019a). Of course, $\beta$-glucan receptors in the gut are not necessarily exclusively expressed on macrophages and could also be found on other locally present (non-) immune cells.

Possibly the most interesting immune-modulating effects of NSP, such as $\beta$ glucans, could be their effect on the innate immune cells associated with the concept of trained immunity, a form of innate immune memory best described in mice and humans. A literature review for indications of trained immunity in fish, supported the notion that the innate immune system of teleost fish can be trained and that effects could be long-lived (Petit and Wiegertjes, 2016). This was based on indications for at least one out of three of the following criteria, considered characteristic of this phenomenon, (i) providing protection against a secondary infection in a T- and B-lymphocyte independent manner; (ii) conferring increased resistance upon re-infection and; (iii) relying on key roles for innate immune cell 
types such as natural killer cells and macrophages. A recent follow-up study in vitro could confirm that common carp macrophages can indeed be trained, with evidence of metabolic reprogramming as well as heightened phagocytosis, production of reactive oxygen species and expression of inflammatory genes (Petit et al., 2019b). Overall, the longevity, non-specificity and presumed evolutionary conservation of immune-modulating effects of NSP associated with trained immunity, could be especially interesting to aquaculture practise.

\section{NSP degrading enzymes}

The use of enzymes, like phytase, xylanase and $\beta$-glucanase in pigs and poultry, is a common way to break down NSP in order to enhance VFA production and to improve the digestibility of the feed (Bedford and Schulze, 1998). Recently, the use of exogenous carbohydrase enzymes in aqua feeds is attracting more attention. Multiple effects have been attributed to exogenous carbohydrase supplementation like improved feed intake, improved growth rate, increase in endogenous digestive enzymes and nutrient digestibility (Goda et al., 2012; Castillo and Gatlin, 2015; Maas et al., 2018). Approximately 200 enzymes from of a microbial origin, out of around 4000 enzymes known, are commercially used. From these 200 enzymes, only 20 are produced on an industrial scale (Li et al., 2012). These include a wide range of carbohydrases, including enzymes that cut part of the polymeric carbohydrates by hydrolysis. Examples of commercially available carbohydrases include; $(\beta-)$ glucanase, ( $a-)$ xylanase, amylase, cellulase, pectinase and galactosidase. Of these enzymes, $\beta$-glucanase and xylanase make up more than $80 \%$ of the commercial market (Adeola and Cowieson, 2011; Castillo and Gatlin, 2015). There is a large variety in NSP (cellulose, arabinoxylans, mixedlinked $\beta$-glucans, mannans, galactomannans, glucomannans, aribans, galactans, arabinogalactans) from different sources, differing in characteristics and properties (Choct and Kocher, 2000; Sinha et al., 2011). NSP have different characteristics and chemical structures, therefore it is likely that not all enzymes have the same effect on diets with different NSP contents. Maas et al. (2019) showed that the effectiveness of phytase and xalanse on growth performance and nutrient digestibility is dependent on the NSP composition. Therefore, it is important that the formulation of the enzyme(s) is complementary to the diet composition (Maas et al., 2019; Officer, 2000). An important benefit of the carbohydrase enzyme is that they reduce the negative effects NSP have on the fish and its gut (i.e., affecting the chyme viscosity). Reducing the negative effects of NSP, and breaking down the polysaccharides into readily available oligomers and monomers for fermentation, will improve nutrient utilisation (Vahjen et al., 2007; Castillo and Gatlin, 2015). 


\section{Concluding remarks}

In this manuscript, an average total carbohydrate ( $\mathrm{CHO}$ ) digestibly of $67 \%$ was calculated for tilapia. The large differences in $\mathrm{CHO}$ digestibility between studies are mainly attributed to the dietary inclusion level of NSP, because NSP is poorly digested compared to starch. NSP digestibility averaged $24.3 \%$ across studies. The presented meta-analysis allows us to assume that NSP digestibility occurred in many tilapia studies ( $88 \%$ ). If we assume that $100 \%$ of the starch was digested, then this would still result in a positive value for NSP digestibility. This clearly demonstrates that NSP is not inert for tilapia but is digestibility. However, between studies and diets the variation in digestibility is high. By knowing the factors affecting NSP digestibility, predictions may be made for NSP ADC. This study shows that NSP ADC increased with increasing fish weights and with dietary carbohydrate levels. Whereas, increasing dietary fat levels, as well as dietary crude fibre levels decreased NSP digestibility. Information on the aforementioned factors enabled a qualitative prediction of NSP digestibility, but for a quantitative prediction of NSP digestibility from dietary and fish related factors, more knowledge is required. In other words, more in vivo studies are required, in which the $\mathrm{CHO}$ and NSP digestibility are actually measured and/or indicators of the degree of fermentation of NSP are used.

In the present study, the $\mathrm{CHO}$ fraction not consisting of starch and sugars was considered as NSP. This approach was used in order to be able to quantify the amount of NSP. However, it is not entirely correct to treat the different types of NSP as one, and to allocate certain properties to NSP as group. As seen in this manuscript, the variation in NSP digestibility is large, which is most likely partly related to the different characteristics of the NSP fractions. In addition, specific NSP, like $\beta$-glucans and MOS, may have immune-modulatory effects and contribute to animal health as a prebiotics. Therefore it is important to create more insight into the effects and characteristics of individual types of NSP.

This study demonstrates that NSP is partially digestible by tilapia, but the process behind it remains unclear. NSP is expected to be fermented through microbial anaerobic glycolysis, forming short chain fatty acids. However, how the glycosidic bonds of the long NSP chains are hydrolysed is unclear, as enzymes like cellulase, ß-xylanases, $\beta$-glucanases and $B$-galactases are scarce, or non-existing, in the GItract of fish. It is unclear if, and to what extent, other fish species can digest NSP. The ability of omnivorous fish to utilise higher levels of carbohydrates, compared to carnivorous fish, is well known. Besides tilapia, Leenhouwers et al. (2007b) showed the ability of African catfish (Clarias gariepinus) to digest NSP with an ADC of up to $56 \%$. Like tilapia, the African catfish is a warm water species adapted to feeding on plant matter. This suggests that NSP is potentially digestible by a wide range of fish species, especially warm water species with a long gut adapted to feeding on plant matter, as these factors favour gut fermentation. 
To conclude, the current meta-analysis confirms the negative effect NSP has on the performance (FCR) and nutrient digestibility (crude protein, fat and energy) of tilapia. NSP is not inert to digestion in tilapia, with an average ADC of $24.3 \%$. The body weight and dietary fat level, total carbohydrates and crude fibre are factors that relate to NSP digestibility, in addition to the functional properties of the NSP fraction, i.e. structure and molecular complexity. NSP could be interesting as functional feed ingredients in aquaculture practice, especially due to NSP associated immune-modulatory effects related to trained immunity. NSP digestibility was shown to contribute to energy digestibility in tilapia. Therefore, in tilapia diet formulations the digestibility, and thus energy originating from NSP, should be taken into consideration as it contributes to the energy needs of the fish.

\section{Acknowledgement}

This work is part of the Netherlands Organisation for Scientific Research (NWO) research programme "Green Aquafeeds" (project number 870.15.100). DuPont Animal Nutrition partly funded the project. 


\section{Supplementary information}

\section{Calculations}

Total carbohydrate digestibility using the mass balance

This approach was used when the ADC of DM, crude protein (CP), fat and ash were reported. The total carbohydrate ( $\mathrm{CHO}$ ) level in the diet on dry mater (DM) basis was calculated as (eq. 1):

$\mathrm{CHO}$ diet $=100-(\mathrm{fat}+$ crude protein + ash $)$

Hereby expressing the dietary fat, CP and ash content in $\mathrm{g} / 100 \mathrm{~g} \mathrm{DM}$.

With the $\mathrm{CHO}$ level the digestibility of $\mathrm{CHO}$ was calculated using the level and ADC of DM, fat, CP and ash in two steps (eq. 2 and eq. 3):

1) $\mathrm{CHO}$ undigested $=(100-A D C D M)-(((A D C$ fat $/ 100) *$ fat diet $)+((A D C$ $C P / 100) * C P$ diet $)+((A D C$ ash / 100 $) *$ ash diet $)))$

2) $\mathrm{ADC} C H O(\%)=100-((\mathrm{CHO}$ undigested / $\mathrm{CHO}$ diet $) * 100)$ (eq. 3)

Where: $\quad \mathrm{CHO}$ undigested $=$ the amount of undigested $\mathrm{CHO}$ in $\mathrm{g} / 100 \mathrm{~g} \mathrm{DM}$ diet This approach allows to calculate the $\mathrm{CHO}$ digestibility when the energy content and $A D C$ of the diet is unknown.

Total carbohydrate digestibility using the energy balance

This approach was used when the ADC of energy, fat and CP was reported. The total carbohydrate $(\mathrm{CHO})$ level in the diet on dry mater (DM) basis was calculated as (eq. 4):

$\mathrm{CHO}=(($ total energy $-(($ Fat $/ 100) * 39.5)-((\mathrm{CP} / 100) * 23.6) / 17.2) * 100$

Hereby expressing the dietary fat, CP and ash content in $\mathrm{g} / 100 \mathrm{~g}$ DM. Energy equivalents of $39.5,23.6$ and $17.2 \mathrm{~kJ} / \mathrm{g}$ were used for fat, $\mathrm{CP}$ and $\mathrm{CHO}$ respectively (NRC 2011).

With the $\mathrm{CHO}$ level the digestibility of $\mathrm{CHO}$ was calculated based on the digestible energy ( $D E$ ) using the $A D C$ of energy, fat and $C P$ in two steps (eq. 5 and eq. 6):

1) $D E$ from $C H O=E$ diet $*(A D C E / 100)-((C P$ in diet $* 23.6 *(A D C C P /$ 100) $)+($ fat in diet $* 39.5 *(A D C$ fat / 100) $))$

2) $\mathrm{ADC} C H O(\%)=(D E C H O /(C H O \operatorname{diet} * 17.2)) * 100$

Where: $\quad E=$ energy

This approach allows to calculate the $\mathrm{CHO}$ digestibility when the ADC of DM and ash is unknown. 


\section{Comparison between methods}

The CHO ADC calculated from DM and energy ADC were highly comparable. An comparison between the $\mathrm{CHO} A D C$ calculated using both methods was done (Figure S1). For one study (Köprücü and Özdemir, 2005) with six diets the difference between both methods was large, with differences up to 33 percentage points. In this study the $\mathrm{CHO}$ ADC calculated from the DM ADC was excluded from the study. This was done as the CHO ADC calculated from the DM digestibility gave questionable values up to $99 \%$, which is extremely high for $\mathrm{CHO}$ not fully consisting of starch. The remaining 53 cases whereby the $\mathrm{CHO}$ ADC was calculated using both methods were highly similar, with a $r^{2}$ for goodness of fit of 0.96 . This high $r^{2}$ value indicates that both way to calculate the CHO ADC could be used interchangeable. When the $\mathrm{CHO} A D C$ was calculated using both methods, the average of the two is used.

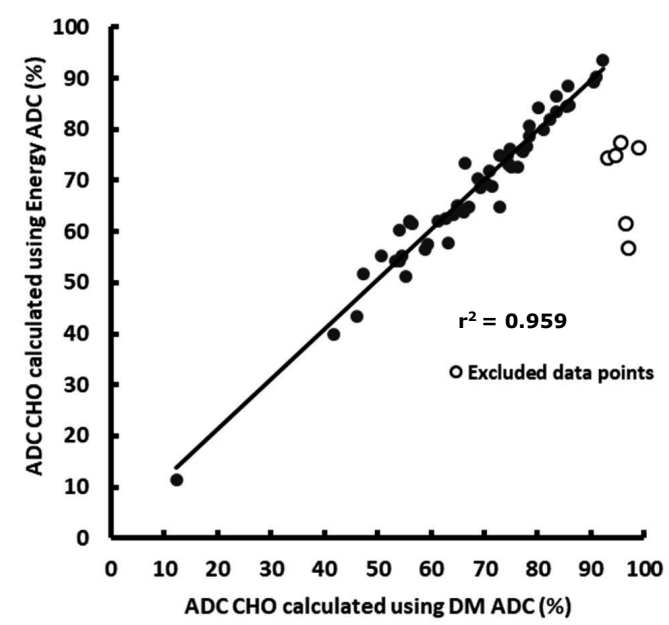

Figure S1 The correlation between the total carbohydrate apparent digestibility coefficient (ADC, \%) calculated from dry matter (DM) and energy digestibility. The estimated regression line with its $r^{2}$ value is given.

\section{Dietary NSP level}

As mentioned in the manuscript, each diet was reformulated with use of the database of the Centraal veevoederbureau (CVB) 2016, in order to calculate the theoretical NSP content. From the theoretical $\mathrm{CHO}$, starch and sugar level the fraction of the NSP was calculated (eq. 7):

NSP fraction $=1-($ starch and sugars $/ \mathrm{CHO})$

The NSP fraction, together with the $\mathrm{CHO}$ level in the diet (equation 1) was used to calculate the NSP level in each diet (eq. 8):

NSP level $=$ NSP fraction $*$ CHO diet 
Cases with a theoretical NSP level of less than $7.0 \%$ (10 in total) were excluded for the calculation of the NSP ADC. This was done as an accurate prediction of the amount of digested NSP with such low NSP contents is disputable.

NSP digestibility

With the NSP fraction and starch and sugar fraction a distinction in the $\mathrm{CHO}$ fraction between highly digestible starch and sugars and poorly digestible NSP was made. To calculate the NSP ADC an assumption for the amount of starch and sugars digested had to be made. As described in the chapter 5. Starch, the ADC is higher for starch when the diet is made by extrusion (99.4\%) compared to steam pelleting ( $91 \%)$. Therefore, a factor of 0.995 for extruded diets and 0.91 for pelleted diets was used for the fraction of starch and sugars digested. With the $\mathrm{CHO}$ ADC, $\mathrm{CHO}$ in the diet, theoretical NSP level in the diet and an factor for the amount of starch and sugars digested, the NSP ADC was calculated in two steps (eq. 9 and eq. 10):

1) Digested NSP $=(\mathrm{CHO}$ diet $* C H O A D C)-(X *$ starch and sugars $)$

2) $A D C N S P(\%)=($ digested NSP / total NSP $) * 100$

Where $X$ is the assumption of the fraction of the starch and sugar digested $(0.995$, extruded diet; 0.91, pelleted diets). 

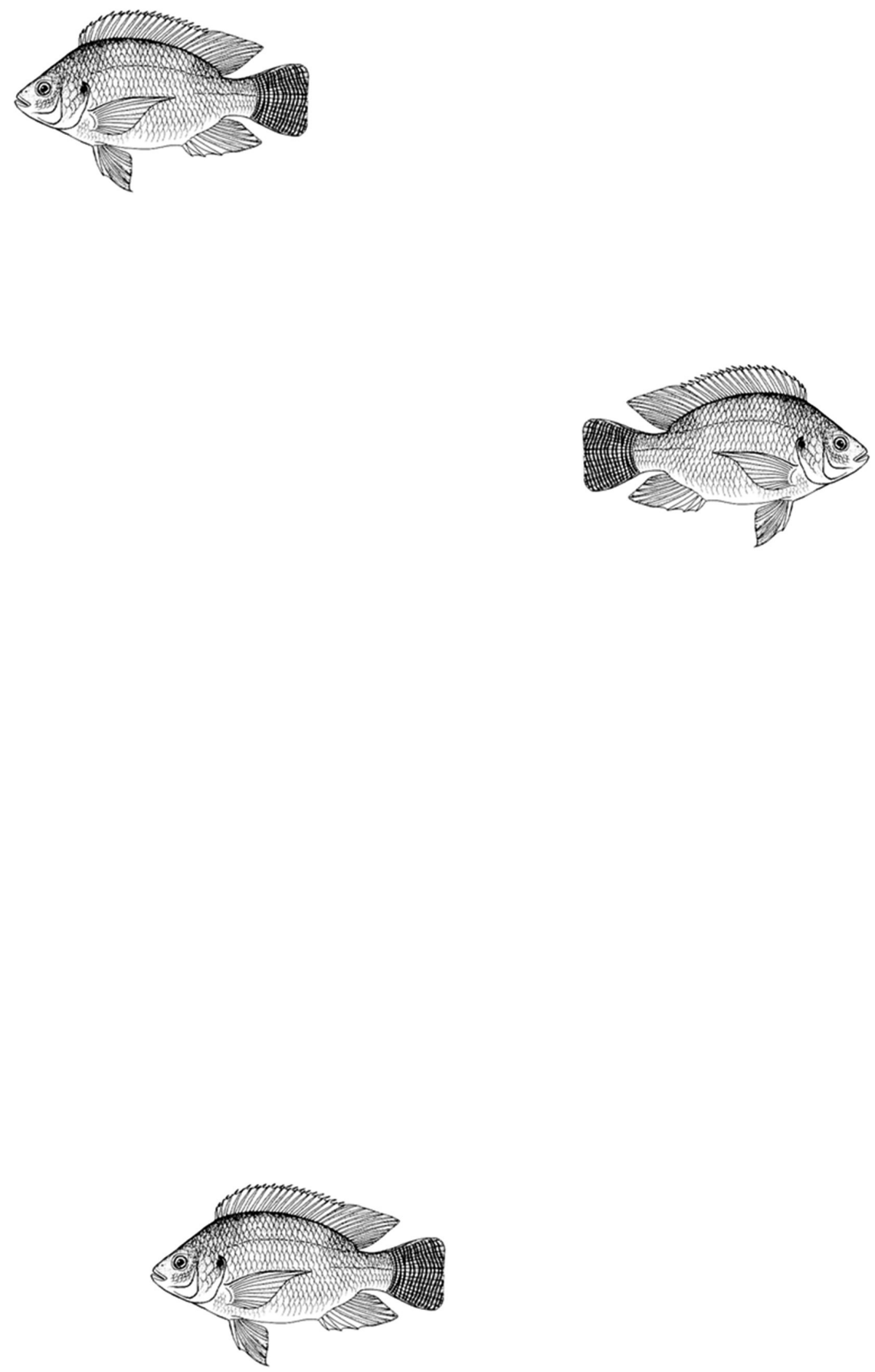


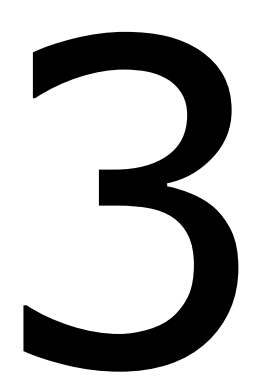

\section{Effect of non-starch polysaccharide composition and enzyme supplementation on growth performance and nutrient digestibility in Nile tilapia}

Roel M. Maas, Marc C.J. Verdegem, Johan W. Schrama

Aquaculture Nutrition (2019), 25: 622-632 


\section{Abstract}

The experiment investigated whether the effect of enzyme supplementation on performance and nutrient digestibility is dependent on type of the ingredient(s) used, by incorporating wheat bran (WB), sunflower meal (SFM) and citrus pulp $(C P)$ to a reference diet (REF). Those ingredients are known to be rich in different types of non-starch polysaccharides (NSP). Diets were supplemented with and without (control) an enzyme mix (phytase $1000 \mathrm{FTU} / \mathrm{kg}$ and xylanase $\mathrm{U} / \mathrm{kg}$ ) according to a $2 \times 4$ factorial arrangement. In total 24 tanks (3 replicates/treatment) were used with 30 fish each (Nile tilapia, Oreochromis niloticus; mean initial body weight $41 \mathrm{~g}$ ). Fish were restrictively fed the experimental diets for 43 days $(80 \%$ of expected satiation). Enzyme supplementation affected the absolute growth $(\mathrm{g} / \mathrm{d})$ and FCR $(P<0.05)$, improving the growth of fish fed the WB and SFM diets, while fish fed the REF and CP diets did not benefit. NSP, energy, ash, phosphorous and calcium digestibility improved with enzyme supplementation $(P<0.05)$. There was an interaction effect on all growth parameters, as well as the digestibility of energy and phosphorus $(P<0.05)$. This indicates that the effectiveness of the enzymes was dependent on the NSP rich ingredient used and thus the composition of the NSP fraction. 


\section{Introduction}

The aquaculture sector expanded rapidly during the last decades, with an annual growth of $5.8 \%$ per year from 2000-2016 (FAO, 2018). The current growth of the aquaculture sector increases the pressure for using more innovative and sustainable ingredients in aqua feeds (Tacon and Metian, 2015). The production of fish meal and fish oil, the conventional protein source in aqua feeds is expected to remain more or less stable the coming years. This partially due to restrictions on the production of fish meal and oil (Hardy, 2010; Mazurkiewicz, 2009; Sinha et al., 2011). The use of plant ingredients is currently increasing, because fish meal replacement is necessary to reduce the feed cost and to keep up with the increasing demand of protein. Therefore, the inclusion levels of dietary fish meal (and fish-oil) in aquafeeds is declining gradually (Carter and Hauler, 2000; Shepherd and Jackson, 2013).

A major downside of using plant ingredient and by products like soya, wheats, sunflower meal, citrus pulp, ryes and legume seeds is the presence of a variety of anti-nutritional substances (ANS), often limiting the inclusion level of these ingredients (Francis et al., 2001). The non-starch polysaccharides (NSP) form one of the major ANS present in plant ingredients. NSP generally remains undigested in monogastric animals as they lack enzymes to break down the long NSP chains. Enzymes that break down NSP such as $\beta$-glucanases and xylanases are limited or not present in the gastrointestinal tract of fish. Therefore NSP is often considered to be of no or little nutritional value (Choct, 1997; Sinha et al., 2011). Another anti-nutritional factor is phytate, which is the main storage form of phosphorus (P). Generally $50 \%$ to $80 \%$ of the $\mathrm{P}$ content in plants is present in the form of phytate. Phytate is unavailable for monogastric animals and as they lack endogenous phytase. Inclusion of dietary phytase can increase the bio-availability of $P$ and reduce the discharge of $P$ to the aquatic ecosystem (Cao et al., 2007; Kumar et al., 2016; Mainstone and Parr, 2002).

Multiple studies in broilers and pigs showed that NSP-containing feedstuff negatively affects the nutrient digestibility and growth (Annison, 1993; Choct and Annison, 1992; JøRgensen et al., 1996; Knudsen et al., 1993; Mroz et al., 2000); in fish similar studies are limited. NSP is known to influence chyme viscosity. Dietary soluble NSP increases the viscosity, thereby decreasing the passage rate and diffusion rates of digestive enzymes and substrates, whereas insoluble NSP increases the passage rate. NSP can influence the physiology of the gut and its function, affecting the endogenous secretion of water, lipids, electrolytes and proteins. Due to these changes, nutrient digestibility and growth performance can be reduced (Choct, 1997; Sinha et al., 2011).

Exogenous enzymes are widely studied and used in pigs and poultry in order to tackle several anti-nutrition factors. Comparable with diet formulation for fish, feed ingredients high in ANS are often included in diet formulation for pigs and poultry for their economically attractive nature (Bedford and Schulze, 1998; Woyengo et 
al., 2014). Commercially, phytase has the largest share, followed by xylanase (Adeola and Cowieson, 2011). Carbohydrates and NSP can be fermented to short chain fatty acids (SCFA) and the gases $\mathrm{H}_{2}, \mathrm{CO}_{2}$ and $\mathrm{CH}_{4}$ through anaerobic microbial glycolysis (Bergman, 1990; Metzler-Zebeli et al., 2010). The SCFA are also referred to as volatile fatty acids (VFA). The principle forms of VFA from carbohydrate fermentation are acetate, propionate and butyrate, which can quickly be absorbed by the colonic lumen (Macfarlane and Macfarlane, 2003; Scheppach, 1994). Leenhouwers et al. (2007b) showed the ability of African catfish (Clarias gariepinus) to partly digest NSP. Hereby the level of digestion was depended on the carbohydrate rich ingredient incorporated in the diet. Similarly, in cows (Russell, 1998) and humans (Macfarlane and Macfarlane, 2003) it is seen that the rate of breakdown of individual NSP differs. Hitherto, little is known about the interaction of NSP and exogenous enzymes in fish. It is expected that the rate of breakdown of different types of NSP depends on the enzyme(s) administered, as individual enzymes target and hydrolyse different bonds. In addition, studies assessing the impact of enzyme supplementation are not always in line with each other. Some studies found improvement in growth performance and nutrient digestibility (Ghomi et al., 2012; Lin, et al., 2007), in contrast other studies found no or minimal effects (Ogunkoya et al., 2006; Yigit and Olmez, 2011). Observed differences among studies could be through a mismatch between the supplied enzymes and the diet, i.e. the NSP composition.

The main objective of the presented study was to determine the effect of type of ingredient, by using ingredients known to be rich in different types of NSP (i.e., cellulose, hemicellulose and pectins) and enzyme supplementation (phytase and xylanase). Main effects and interaction between the applied enzymes and the type of ingredient on the growth performance and nutrient digestibility in Nile tilapia (Oreochromis niloticus) were tested.

\section{Material and methods}

\section{Diets}

Eight diets were formulated according to a $2 \times 4$ factorial arrangement (Table 1 ). The first factor altered was the NSP composition by incorporating wheat bran (WB), sunflower meal (SFM) and citrus pulp (CP) to a reference diet (REF). The reference diet was used as the control diet. The ingredients were chosen for their high NSP content and their contrast in NSP composition; WB being relative rich in hemicellulose, SFM in cellulose and CP in pectins (Knudsen, 1997; Pascoal et al., 2012; Rodrigues et al., 2012). Different inclusion levels of the NSP rich ingredients were applied in order to keep the theoretical NSP content similar. Therefore, the inclusion levels into the REF diet were $25.5 \%$ for WB, $27.5 \%$ for SMF and $20 \%$ for $\mathrm{CP}$. The second factor is diets supplemented with an enzyme cocktail containing $4000 \mathrm{U} / \mathrm{kg}$ xylanase (Danisco ${ }^{\circledR}$ Xylanase, DuPont Animal Nutrition) and 1000 FTU/kg phytase (Axtra ${ }^{\circledR}$ PHY, Buttiauxella sp. phytase, DuPont Animal Nutrition), versus a control (no enzymes added). The enzyme supplementation was successful 
with the indented recovery. Diets with enzymes had an average measured phytase activity of $915 \mathrm{FTU} / \mathrm{kg}$ DM and diets without enzymes <50 FTU/kg DM (Table 2). The xylanase activity was only measured for the diets with enzymes, measuring an average activity of $3871 \mathrm{U} / \mathrm{kg}$ DM. The experimental diets were formulated to meet the requirements of the essential nutrients according to the NRC (2011). Diets were supplemented with the essential amino acids, lysine, methionine and threonine. It was intended to keep the diets isonitrogenous (Table 2), therefore casein was added to the WB and CP diets to compensate for the lower protein content of these ingredients. Yttrium oxide $\left(\mathrm{Y}_{2} \mathrm{O}_{3}\right)$ was included (at $0.02 \%$ ) as inert marker for digestibility studies. The analysed nutrient composition of the experimental diets are given in Table 2.

Diets ( $3 \mathrm{~mm}$ pellets) were produced by Research Diet Services (Wijk bij Duurstede, The Netherlands), by extrusion. Fat and enzymes were added together to the diets after extrusion by vacuum coating (Vacuüm core coater, Pegasus ${ }^{\circledR}-10$ VC, 1/4 H/VV nozzle nr. 6502), at the research facilities of the Animal Science Group, Wageningen University, The Netherlands. A mixture of fish oil, rapeseed oil and palm oil in a ratio of 2:5:5 was applied to add an additional $6 \%$ fat, resulting in approximately $8 \%$ dietary fat. The REF diet was extruded with $1 \%$ fish oil and $2.5 \%$ of rapeseed oil, as extrusion without added oil failed to produce a stable pellet. The remaining oil (palm oil) was coated following the same procedure as the other diets. The coating allowed for the addition of enzymes, using liquid forms with the fat to the diets. The enzymes were diluted 1:50 with demineralised water to increase the volume and to ensure a more homogenous dispersal of the enzymes during coating. For the treatments containing enzymes, 5-ml per $\mathrm{kg}$ feed of solution was added to the oils to achieve the above mentioned enzyme activities. Control diets (no enzymes) were coated with 5-ml demineralised water per $\mathrm{kg}$ feed. Diets were stored at refrigerated conditions $\left(4{ }^{\circ} \mathrm{C}\right)$ throughout the experiment. Prior to coating the diets were stored in the freezer at $-20{ }^{\circ} \mathrm{C}$.

\section{Fish, rearing conditions and housing facilities}

This experiment was evaluated by the Animal Welfare Body of Wageningen University and evaluated as not being an animal experiment according to Dutch legislation (Act on Animal Experiments).

The experiment was conducted in the Aquaculture Research Facility (ARF) of the Wageningen University, The Netherlands. Nile tilapia (Oreochromis niloticus) from an natural male silver strain (Silver $\mathrm{NMT}^{\mathrm{TM}}$ ) were obtained from a commercial fish breeder (Til-Aqua international, Someren, The Netherlands). Fish were housed in 160-L tanks and fed a commercial diet (ME-1.8 MP Presta, containing 55\% protein and $15 \%$ fat, Skretting, Stavanger, Norway) prior to the start of the experiment. 


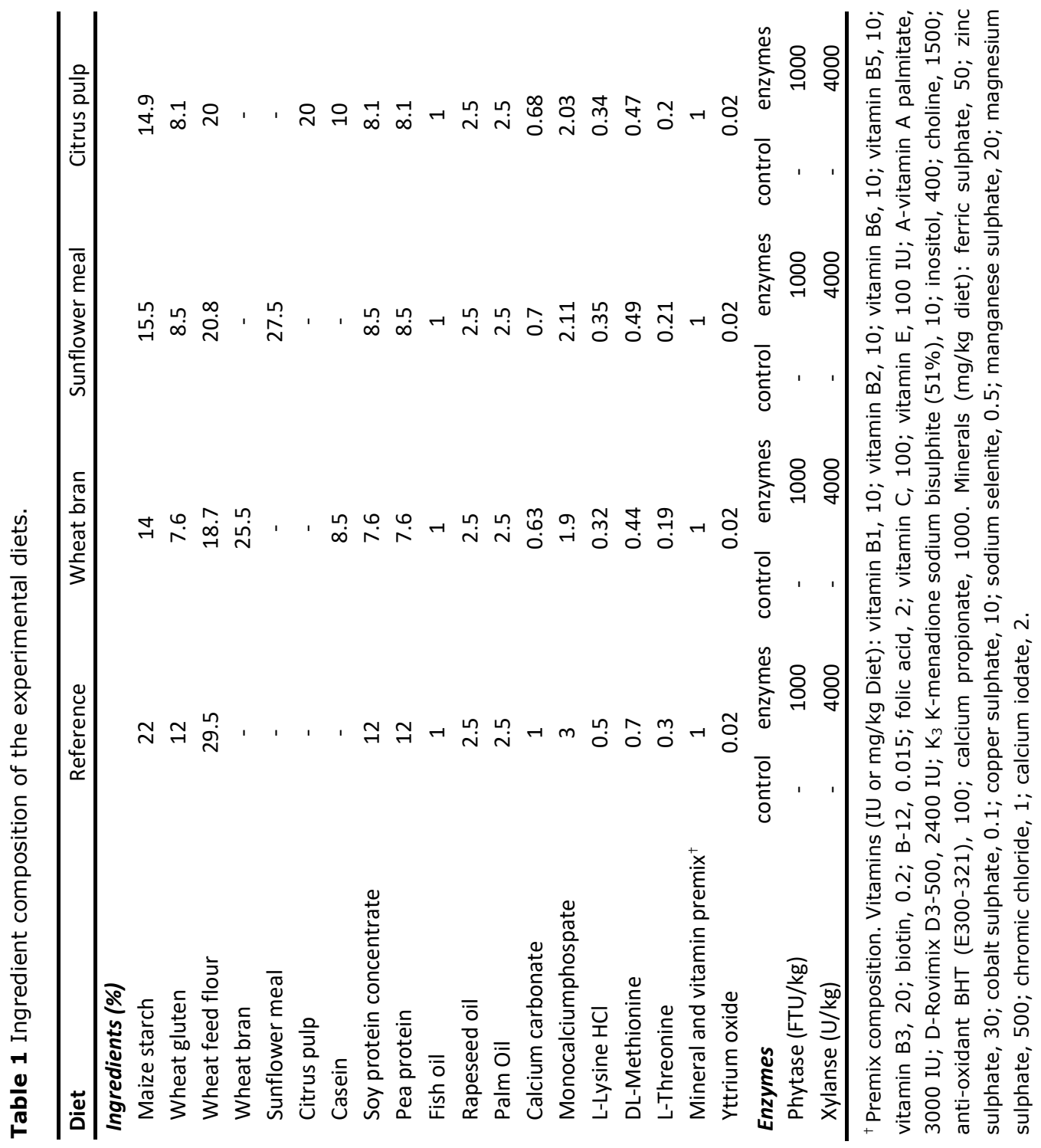




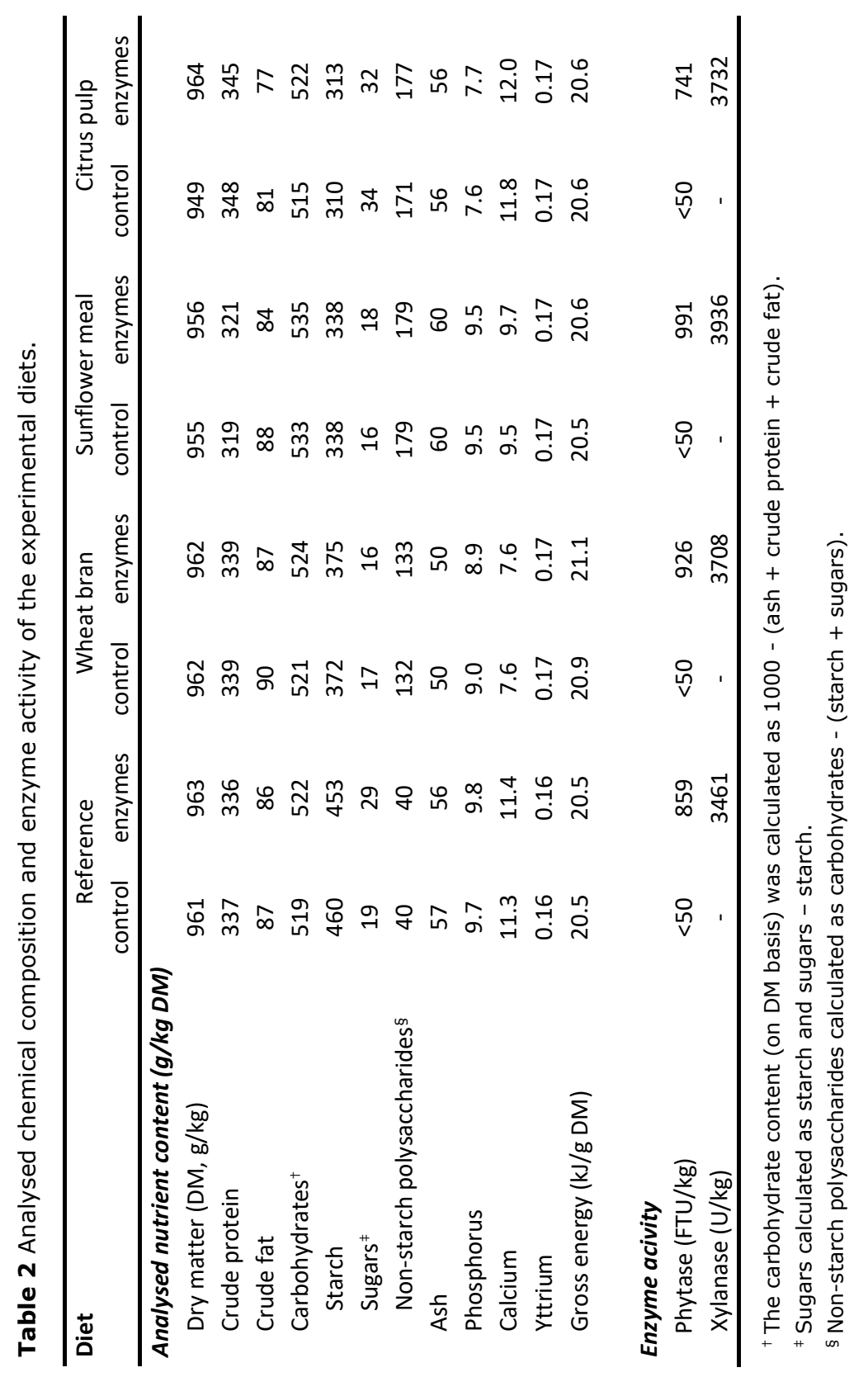


In total 24 rectangular tanks of 60-L placed in three rows of eight were used. Each tank was stocked with 30 fish, having an average start weight of $41.1 \mathrm{~g}$. The tanks were all connected to the same recirculation system resulting in a common water supply, ensuring the same water quality for the inflow of each tank. The water refreshment of the system was $300 \mathrm{~L} /$ day. The recirculation system consisted of a sump, settling tank and trickling filter. Every single tank was connected to a swirl separator (AquaOptima AS, column height $44 \mathrm{~cm}$; diameter $24.5 \mathrm{~cm}$ ) with a detachable glass bottle at the bottom, in order to count feed spills and collect faeces for each tank separately. The flow through each tank was set at $7 \mathrm{~L} / \mathrm{min}$ with a hand held liquid rotameter. All tanks were provided with a cylinder shaped air stone. The air stone and water flow ensured sufficient dissolved oxygen (DO) levels. The photoperiod was set to $12 \mathrm{~h}$ light: $12 \mathrm{~h}$ dark (lights on 7:00, lights off 19:00).

Water quality parameters were measured three times per week in the morning before feeding to ensure that the water quality parameters remained within the pre-set ranges. The temperature (Testo 110 ) range was $27.4-27.6{ }^{\circ} \mathrm{C}$. The $\mathrm{pH}$ (WTW-pH 325) range was set between 7.0 and 7.8, sodium bicarbonate was added when the $\mathrm{pH}$ tended to drop below 7.0. The $\mathrm{pH}$ ranged between 6.7 and 7.8 during the experiment. DO levels of the common outflow ranged between 6.1 and 7.6 mg/L (WTW-Oxi 340i). The conductivity (WTW-Multi 3430) was kept between 6.6$10.6 \mathrm{mS} / \mathrm{cm}$, comparable to the conditions prior to the experiment. The concentration of total ammonia nitrogen (TAN, Merck Aquamerck Colorimetric Ammonium test), nitrite (NO2, Merck Aquamerck Colorimetric Nitrite test) and nitrate (NO3, Merck MQuant Nitrate test strips) were measured. Total ammonia levels of the outflow remained below $0.3 \mathrm{mg} / \mathrm{L}$, nitrite $<0.2 \mathrm{mg} / \mathrm{L}$ and nitrate $<500$ $\mathrm{mg} / \mathrm{L}$ respectively.

\section{Experimental procedure}

A feeding trail was conducted for 6 weeks (43 days). At the start 30 fish were randomly stocked per tank, fish per tank were batch weighed to determine the initial weight. At the end of the experiments all fish per tank were batch weighted to determine the final weight and to calculate growth parameters. The eight experimental diets were assigned randomly per rack of eight tanks. This resulted in 3 replicates per treatment.

The aim within the experiment was to test the effect of the applied enzymes and carbohydrate source on growth and digestibility. Therefore, the fish were restrictedly fed to keep the equal amount of feed on dry matter (DM) basis per tank per day. The feeding level was fixed at $16 \mathrm{~g} / \mathrm{kg}^{0.8} / \mathrm{d}$, about $80 \%$ of the expected satiation level. The daily amount of feed was increased throughout the experiment by predicting fish growth and weight, using the average start weight of the fish and an expected feed conversion ratio of 1.0. At the first feeding moment the fish were fed $20 \%$ of the intended feeding level, which was stepwise increased during 12 days to $100 \%$. The feeding level was stepwise increased to 
allow the fish to adapt to the experimental diets and to prevent feed spills and refusals. The daily feed ration was divided into two equal portions fed at 9:00 and 15:30 $\mathrm{h}$. The fish were hand fed, feeding did not exceed $1 \mathrm{~h}$ for all tanks. Fifteen min after feeding, the settling bottles connected to the swirl separators were emptied and checked for uneaten pellets. The feed was sieved to remove small particles and dust and stored in plastic buckets in the fridge at $4{ }^{\circ} \mathrm{C}$. Each week a feed sample of $100 \mathrm{~g}$ was taken from each diet, this pooled sample was stored in the fridge at $4{ }^{\circ} \mathrm{C}$ until further analysis.

Faeces were collected for digestibility studies using a swirl separator, with a detachable glass bottle $(500-\mathrm{ml})$. The glass bottles were submerged in ice to prevent bacterial degradation of the faeces. Faeces were collected between the afternoon and morning feeding (16:30 $\mathrm{h}-8: 00 \mathrm{~h})$. Faeces were pooled per week and stored in aluminium trays at $-20{ }^{\circ} \mathrm{C}$ until further analysis.

\section{Analyses}

Collected faeces from the last 17 days were dried at $70{ }^{\circ} \mathrm{C}$ and pooled per tank. The faeces were ground using a mixer mill (Retsch Brinkmann, model MM2000) prior to the analysis. The diets were analysed as whole pellets. Collected faeces and feed were analysed for DM by drying at $103{ }^{\circ} \mathrm{C}$ for $4 \mathrm{~h}$ until constant weight. Ash content was determined by incineration in a muffle furnace for $4 \mathrm{~h}$ at $550{ }^{\circ} \mathrm{C}$ (ISO 5984, 1978). The total nitrogen content was measured using the Kjeldahlmethod (ISO 5983), calculating crude protein as $N \times 6.25$. Crude fat was measured by petroleum-ether extraction (Soxhlet method, ISO 6492) and energy by bomb calorimeter (IKA ${ }^{\circledR}$ werke, C7000; IKA analysentechnik, Weitershem, Germany). Starch in feed and faeces was externally determined enzymatically using amyloglucasidase and starch + sugars in feed using the same method without the ethanol extraction measuring glucose units, including those originating from free sugars. Yttrium, $\mathrm{P}$ and $\mathrm{Ca}$ were analysed using inductively coupled plasma-mass spectrometry (ICP-OES) according to the standard NEN 15510 (2007).

Danisco Animal Nutrition Laboratories (Brabrand, Denkmark) analysed the feed samples for phytase activity as described by Yu et al. (2012) and for xylanase activity as described by Romero et al. (2013). 


\section{Calculations}

Growth performance was calculated as described by Saravanan et al. (2012). The absolute growth (in $\mathrm{g}$ ) was calculated as the difference between the average individual initial $\left(W_{i}\right)$ and final $\left(W_{f}\right)$ body weight per fish. The geometric mean body weight ( $W_{G}$ in $g$ ) was calculated as $V\left(W_{f} \times W_{i}\right)$ and the mean metabolic body weight $\left(\mathrm{MBW}_{\mathrm{G}} \text { in } \mathrm{kg}^{0.8} \text { ) as (WG / 1000) }\right)^{0.8}$. The growth rate on metabolic weight (GR mbw in $\left.\mathrm{g} / \mathrm{kg}^{0.8} / \mathrm{d}\right)$ was calculated as $\left(W_{f}-W_{i}\right) /\left(M B W_{G} \times t\right)$, where $t$ is the duration of the experiment in days (d). The daily growth coefficient (DGC) was calculated as $100 \times\left(\mathrm{W}_{\mathrm{f}}^{1 / 3}-\mathrm{W}_{\mathrm{i}}^{1 / 3}\right) / \mathrm{t}$. The daily absolute feed intake $\left(\mathrm{FI}_{\text {abs }}\right.$ in $\mathrm{g} \mathrm{DM} /$ fish/d) was calculated as $\mathrm{FI}_{\text {tot }} /(\mathrm{n} \times \mathrm{t})$, were $\mathrm{n}$ is the number of fish per tank and $\mathrm{FI}$ tot the total feed intake (in $g \mathrm{DM}$ ), corrected for dead fish and feed spills. The feed conversion ratio (FCR) was calculated as $\mathrm{FI}_{\mathrm{mbw}} / \mathrm{GR}$ mbw, where the $\mathrm{FI}_{\mathrm{mbw}}$ is the feed intake per metabolic bodyweight (in $\mathrm{g} \mathrm{DM} / \mathrm{kg}^{0.8} / \mathrm{d}$ ) calculated as $\mathrm{FI}_{\text {abs }} / \mathrm{MBW}_{\mathrm{G}}$.

Yttrium was used as inert marker to calculate the apparent digestibility coefficient (ADC in \%) of dry matter, crude protein, crude fat, total carbohydrate, starch, NSP, gross energy, ash, P and calcium (Ca) for each tank. The ADC was calculated according to the following formula (Cheng and Hardy, 2002): ADC (\%) $=100 \times[1$ - ( $Y_{i} \times$ amount nutrient in faeces) / ( $Y_{f} X$ amount nutrient in feed $\left.)\right]$, where $Y$ is the concentration of yttrium in the diet and faeces and $\mathrm{N}$ is the quantity of nutrient or energy content in the diet an faeces. The total amount of carbohydrates in feed and faeces was calculated on DM basis as: 1000 - (crude protein + crude fat + ash). The NSP fraction was calculated as: total carbohydrates - (starch + sugars).

\section{Statistical analysis}

A two-way ANOVA using a General Linear Model (GML) was used for the effect of enzyme supplementation, dietary treatment and their interaction on all data. When interaction was found $(P<0.05)$, a Tukey HSD (honest significant difference) with multiple comparison with $95 \%$ level of significance was used to compare treatment means. Data of performance and digestibility were expressed as the mean per treatment of three replicates. All statistical analyses were carried out using the IBM Statistical Package for the Social Sciences (SPSS) program (v 23.0, New York, United States). 


\section{Results}

\section{Performance}

Mean fish performance is given in Table 3 . The overall survival of the 43 day experiment was high with $99.2 \%$ and was unaffected by the dietary treatments. As intended, the absolute feed intake $(1.33 \mathrm{~g} \mathrm{DM} / \mathrm{d})$ was equal among treatments. All growth parameters had a diet effect $(P<0.001)$, with the best growth for the REF diets low in NSP content. The lowest growth was for the SFM diets. The enzyme supplementation positively affected the absolute growth $(\mathrm{g})$ and the FCR $(P<0.05)$. There was an interaction effect between diet and enzyme supplementation on all growth parameters $(P<0.05)$, indicating that the effectiveness of the enzyme was depending on the test ingredient used and thus the NSP composition in the diet. The enzyme supplementation resulted in a better performance for the fish fed the WB and SFM diets, improving the FCR from 1.00 to 0.92 for the WB diet and from 1.12 to 1.07 for the SFM. While the REF diet (low in NSP) and the CP diet did not benefit from the enzyme supplementation.

\section{Digestibility}

The test ingredients (WB, SFM and CP) affected the ADC of all nutrient $(P<0.001)$, except for the starch and $\mathrm{Ca}$ (Table 4). Starch was almost completely digested with an ADC of $99.9 \%$ for all diets. The incorporation of the test ingredients high in NSP content reduced the nutrient digestibility compared to the REF diet low in NSP, except for the ash digestibility. The supplementation of phytase and xylanase enhanced the digestibility of the ash fraction and the minerals $\mathrm{P}$ and $\mathrm{Ca}(P<0.05)$. The digestibility of the crude protein, crude fat and total carbohydrate fraction remained unaffected by the enzyme supplementation $(P>0.05)$. However, the energy digestibility, which is by definition the sum of digested energy from crude protein, crude fat and total carbohydrate, was affected by the applied enzymes $(P<0.05)$. The NSP fraction was not inert for digestion and improved with enzyme supplementation $(P<0.05)$. The ADC of NSP fraction improved from 17.1 to $25.1 \%$ for the WB diet, from 18.8 to $22.3 \%$ for the SFM diet and from 31.7 to $32.9 \%$ for the CP diet. The NSP digestibility is in line with the growth performance, where the effect of the enzymes supplementation was the largest for the WB diet followed by the SFM diet, while being minimal for the CP and REF diets. There was an interaction effect between the applied enzymes and the diet for the energy and $\mathrm{P}$ digestibility $(P<0.05)$. Similar with the NSP digestibility the increase in energy and $P$ digestibility was the largest for the WB and SFM diets. With enzyme supplementation the $P$ digestibility increased with 4, 15, 13 and 2 percentage points for the REF, WB, SFM and CP diets, respectively. 


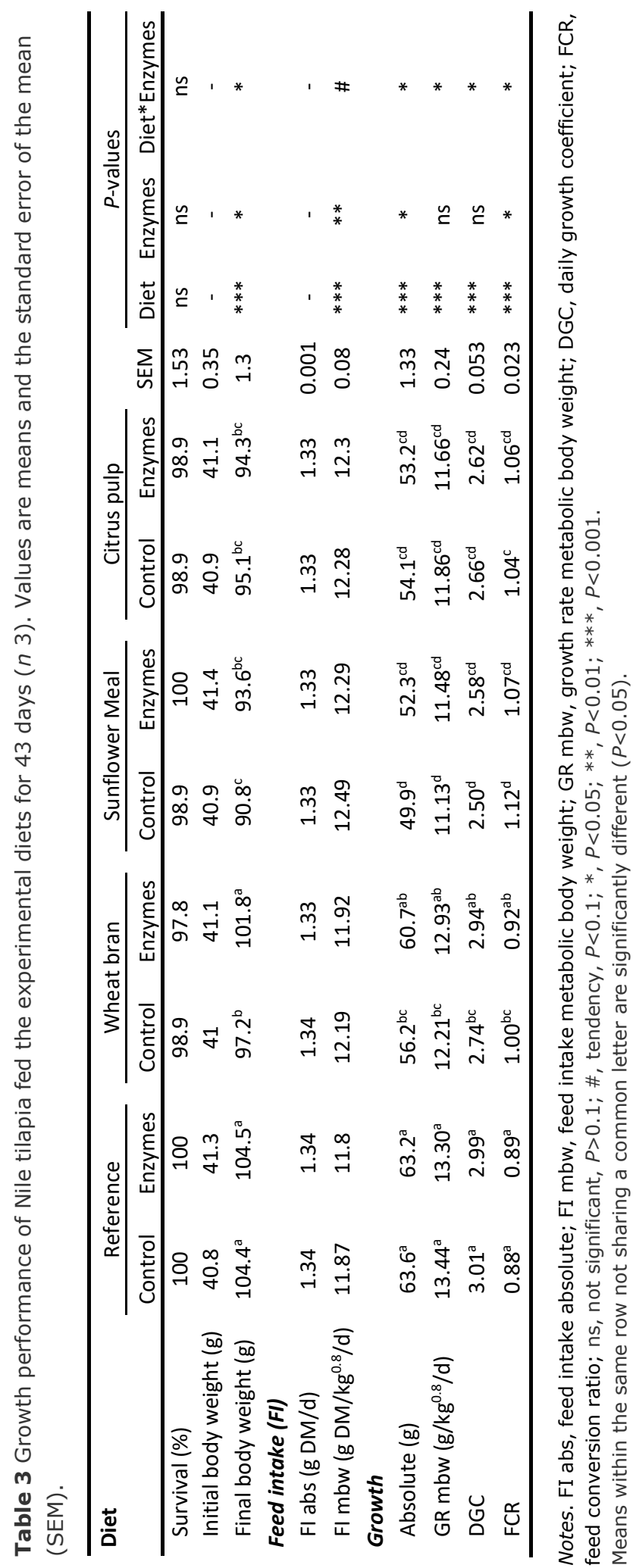




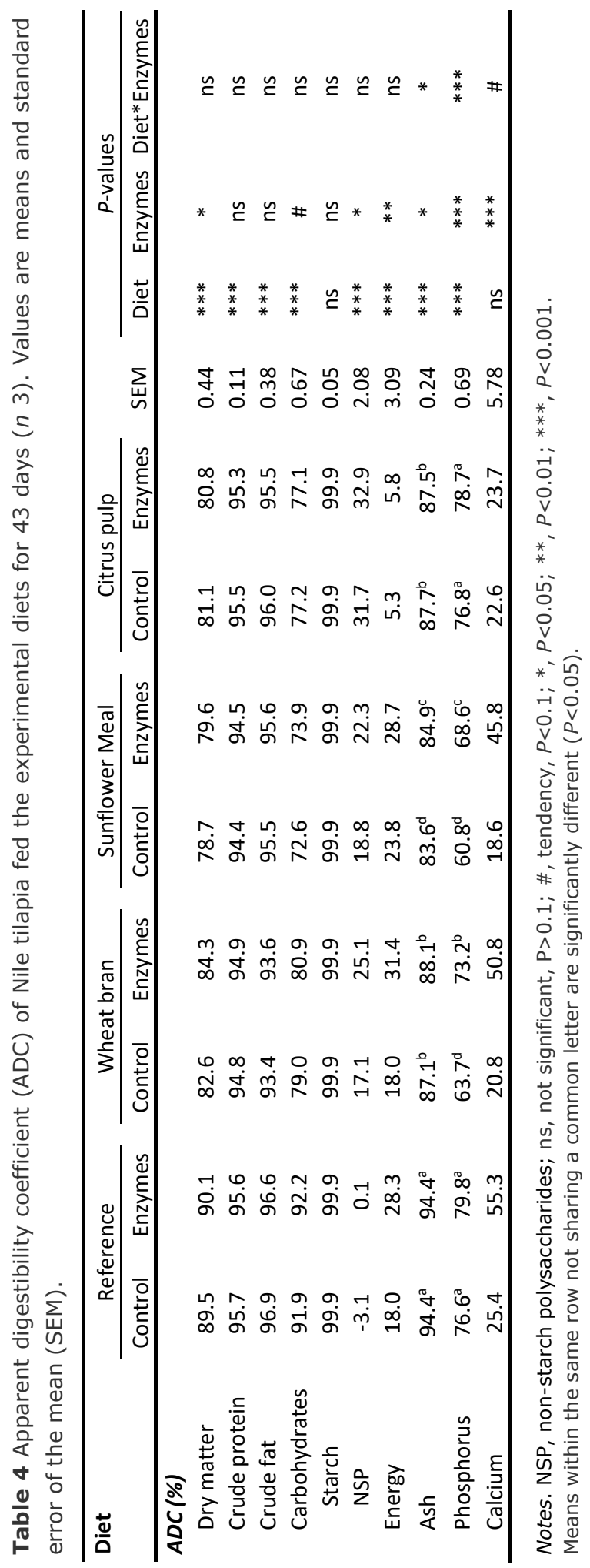




\section{Discussion}

The experiment investigated whether the effect of enzyme supplementation on nutrient digestibility and growth dependents on the type of carbohydrate, by incorporating ingredients contrasting in carbohydrate (NSP) composition. The diets were comparable in nutrient composition except for the type of NSP and the level of NSP, which differed slightly (Table 2). The inclusion of the NSP rich test ingredients reduced the growth performance compared to REF diet low in NSP content, as seen in previous studies (Haidar et al., 2016; Leenhouwers et al., 2007a). The FCR (0.88) of the fish fed the REF diet (control) was relative low in the current study when comparing with results of other studies (Amirkolaie et al., 2006; Haidar et al., 2016; Leenhouwers et al., 2007a; Maas et al., 2018; Saravanan et al., 2012; Schneider et al., 2004; Schrama et al., 2012) performed at the same facilities (ARF, Wageningen University). The FCR for the NSP rich control (no enzymes) were 1.00, 1.12 and 1.04 for the WB, SFM and CP diets, respectively. With the calculated NSP levels in the diet (Table 2 ) and the ADC of NSP (Table 4), the amount of undigested NSP of each of the control diets was derived as 40,109, 145 and $118 \mathrm{~g} / \mathrm{kg}$ DM feed for the REF, WB, SFM and CP diets respectively. It can been seen that the level of undigested NSP affected the growth, i.e., the FCR increased with the level of undigested NSP.

The inclusion of the NSP rich ingredients had an effect on the ADC of all nutrients, DM and energy, except for the starch digestibility which was almost complete for all treatments $(99.9 \%)$. The reduced growth for the fish fed the NSP rich diets is reflected in a numerically lower fat, crude protein, total carbohydrate, DM, energy and $\mathrm{Ca}$ digestibility. This could be expected due to the assumed negative effects of NSP on the gut physiology and its function leading to a decrease in nutrient digestibility and growth performance (Choct, 1997; Sinha et al., 2011). In addition, higher levels of phytate are known to reduce the bio-availability of minerals and to form phytic acid-protein complexes (Francis et al., 2001; Nolan et al., 1987). Haidar et al. (2016) showed comparable results for a diet high in NSP compared to a diet low in NSP (high in starch), whereby the fat, crude protein, total carbohydrate, DM and energy digestibility were numerically lower when feeding until satiation or a medium feeding level $\left(11.3-11.6 \mathrm{~g} / \mathrm{kg}^{0.8} / \mathrm{d}\right)$. The presented study shows that NSP is not inert for digestion in Nile tilapia with an endogenous NSP digestibility ranging from -3.1 up to $32.9 \%$ (Table 4). The available literature about NSP digestibility is scarce, but showed that NSP can be partly digested in Nile tilapia (Amirkolaie et al., 2005; Haidar et al., 2016; Leenhouwers et al., 2007a) and in African catfish (Leenhouwers et al., 2007b), although long considered indigestible as fish lack the enzymes to break down the long polysaccharide chains of NSP (Choct, 1997; Sinha et al., 2011). The NSP digestibility showed to be depending on the NSP type (Amirkolaie et al., 2005; Leenhouwers et al., 2007a; Leenhouwers et al., 2007b) and feeding level (Haidar et al., 2016). The current study showed that the NSP digestibility was dependent on the test ingredient used to elevate the NSP levels in the diet (Table 4). This is in line with Leenhouwers et al. (2007a), where the incorporation of $40 \%$ of four 
different grains (maize, wheat, barley and rye) resulted in differences in NSP digestibility. There were no purified forms of different NSP used (i.e. cellulose) in the diets, therefore the NSP content in the diets contained a mixture of different types of NSP. However, with the different ingredients a contrast in NSP type was created, with WB being relative rich in hemicellulose, SFM in cellulose and CP in pectins respectively (Knudsen, 1997; Pascoal et al., 2012; Rodrigues et al., 2012). The pectin rich $\mathrm{CP}$ diet showed the highest endogenous NSP digestibility with $31.7 \%$ followed by the SFM (18.8\%) and WB (17.1\%) diets, with a comparable digestibility. The solubility of the NSP fraction is an important factor affecting the digestibility of the NSP fraction (Leenhouwers et al., 2007a; Sinha et al., 2011). Leenhouwers et al. (2007a) made a distinction between soluble and insoluble NSP digestibility in Nile tilapia, indicating negative values for the digestibility of the insoluble NSP fraction and an ADC up to $60 \%$ for the soluble fraction. Comparable with Nile tilapia, the digestibility of the soluble NSP fraction was higher than the insoluble fraction in African catfish, independent of diet composition (Leenhouwers et al., 2007b). The NSP coming from the CP contains theoretically a higher percentage ( $72 \%$ ) of soluble NSP compared to WB ( $8 \%$ ) and SFM (18\%) (Knudsen, 1997; Pascoal et al., 2012). The higher percentage of soluble NSP in the CP diets could explain the higher endogenous NSP digestibility.

The enzymes phytase and xylanase affected the absolute growth $(\mathrm{g} / \mathrm{d})$ and the FCR. The enzymes improved the FCR from 1.00 to 0.92 for the WB diet and from 1.12 to 1.07 for the SFM diet. However, the REF diet low in NSP and the pectin rich $\mathrm{CP}$ diet did not benefit from the enzyme supplementation. The enzyme supplementation had an effect on the ADC of energy, DM, NSP, ash and the minerals $\mathrm{Ca}$ and $\mathrm{P}$. In line with the growth performance the enzyme supplementation had the largest effect on the ADC of NSP for the WB diets (17.1 to $25.1 \%$ ) followed by the SFM diets (18.8 to $22.3 \%$ ), while being minimal for the CP diets (31.7 to 32.9\%). Similar the ADC of P increased with 4, 15, 13 and 2 percentage points for the REF, WB, SFM and CP diets respectively. The increase in NSP, $P$ and energy digestibility caused the improvement in growth for the fish fed the WB and SFM diets. While the minimal effect of the enzymes on the digestibility of the REF and CP diet did not result in enhanced growth.

The limited amount of literature on carbohydrases and their effect on nutrient digestibility and performance in fish is not always coherent. In most studies the enzymes improved the nutrient digestibility and performance (Adeoye et al., 2016a; Ghomi et al., 2012; Lin et al., 2007; Maas et al., 2018; Yildirim and Turan, 2010), while the effect was absent or minimal in other studies (Ogunkoya et al., 2006; Yigit and Olmez, 2011). The present study shows both, with a positive effect of the enzymes on the WB and SFM diets, while there was no effect on the CP diet. Therefore, it is important to have insight in diet composition and the substance targeted with the use of enzyme supplementation. There is a large variety of NSP (cellulose, arabinoxylans, mixed-linked $\beta$-glucans, mannans, galactomannans, glucomannans, aribans, galactans, arabinogalactans) differing in characteristics, chemical structures and properties (Choct, 1997; Sinha et al., 2011). Therefore, it 
is likely that not all enzymes have to same effect on diets differing in NSP composition and is it important that the enzyme(s) supplementation is complementary with the diet composition (Officer, 2000). Xylanase is an carbohydrase which hydrolyses the $\beta-1,4$ xylan, the xylan backbone of arabinoxylans into its constituent sugar xylose (Beg et al., 2001). WB is rich in hemicellulose, with arabinoxylans being the major polysaccharide present (Choct, 1997; Englyst, 1989). Englyst (1989) measured the constituent sugars of NSP by gas-liquid chromatography, for WB $45 \%$ of the total NSP consisted of xylose. With approximately $75 \%$ of the NSP in the WB diet originating from WB (Table 1 ), theoretically at least 33\% of the NSP should be in the form of arabinoxylans. With the increase in NSP digestibility from 17.1 - to $25.1 \%$ with enzyme supplementation, it is most likely that the breakdown of the present arabinoxylans was incomplete. Both SFM and CP contain considerable amounts of hemicellulose and arabinoxylans, although being relatively rich in cellulose (SFM) and pectins (CP) (Englyst, 1989; Pascoal et al., 2012). The enzymes were effective to increase the growth and NSP digestibility (18.8- to 23.2\%) for the SFM diet, while growth was unaffected for the CP diets with a minimal increase in NSP digestibility (31.7to $32.9 \%$ ). The level and structure of hemicellulose (in particular the arabinoxylans) as well as the endogenous digestibility of the hemicellulose fraction could explain for the differences found between the SFM and CP diets.

The implementation of the use of products like enzymes on a commercial scale in fish feeds depends on the economic profitability. The extra cost due to enzyme supplementation should be lower than the extra income generated. The extra income generated by enzyme supplementation depends on the improvement in growth (i.e. FCR) and the market price of tilapia. In this study, enzyme supplementation had the largest reduction in FCR at the WB diet from 1.00 to 0.92 (Table 3.), which implies that enzyme supplementation resulted in $8.7 \%$ extra growth per $\mathrm{kg}$ feed. The price of tilapia fluctuates over time and depends on: fish quality; the product form (whole fish, frozen fillets, etc.); and the location of production/sale. E.g. a price of $1.53 \mathrm{US} \$ / \mathrm{kg}$ life fish was reported to be paid in Brazil in January 2018 by a fish processor (de Oliveira Coutinho et al., 2018). Farm gate (2018) reported average tilapia raw material prices of $1.40 \mathrm{US} \$ / \mathrm{kg}$ in the Guangdong region (China) in March 2018. The dose of the liquid enzyme cocktail (phytase and xylanase) used in this study was $1 \mathrm{~kg}$ per 5 MT feed. Using the aforementioned prices of tilapia and FCR of the WB diet (1.00), enhanced growth increases the value of the crop by 609 to 665 US\$ per kg of enzyme cocktail $(5.000$ $\mathrm{kg}$ feed $* 0.087 \mathrm{~kg}$ extra live weight tilapia per $\mathrm{kg}$ feed $* 1.40$ or 1.53 US\$ per kg fish). The cost price of the $1 \mathrm{~kg}$ enzyme cocktail is estimated to be below $20 \mathrm{US} \$ / \mathrm{kg}$ thus yielding more than 30-fold return on investment. It will depend on the cost benefit margin realised by the farmer per $\mathrm{kg}$ tilapia sold, whether adding the enzyme cocktail to the diet is cost effective. This calculation does not take into account the financial benefit of having a shorter production cycle due to the improvement in growth using the same amount of feed, nor the cost of enzymes application. It should however be realised that the profitability of enzyme 
supplementation is dependent on the diet composition. With the high digestible REF diet, the FCR was not altered and thus gave zero economic benefit.

In practice, tilapia is mostly cultured in ponds and sometimes in cages. This study was performed in glass aquaria connected to a recirculation aquaculture system without a nature food web. Kabir et al. (2019) showed that in ponds up to $64 \%$ of the protein gain originates from protein from the natural food web and that especially low quality feeds can stimulate the natural food web. It was suggested that the natural food web in the ponds have a large contribution to the growth of tilapia especially when fed a sub-optimal protein to energy ratio. In a pond setting, adding enzymes to the feed might improve the food utilisation by the fish, but it is not clear if this will alter the enhancement of the productivity of the natural food web. In other words, the positive effect of enzymes in a ponds system may be altered by effects on the natural food web productivity. Therefore, further research about the practical implications of enzymes in both pond and cage cultures is recommended before applying on large commercial scale.

This study showed that the supplementation of phytase and xylanase can partly reduce negative effects induced by the use of NSP and phytate rich ingredients on nutrient digestibility and growth. The extra generated income with the use of enzymes looks promising, however the translation to large scale commercial systems is still required. An interaction was observed between dietary ingredient composition and enzyme supplementation on the growth parameters and on the $P$ and energy digestibility. This indicates that the effectiveness of the applied enzymes is dependent on the NSP rich ingredient used and thus the composition of the NSP fraction.

\section{Acknowledgements}

We thank the staff of the aquaculture research facilities for their technical support in conducting the experiment and Ronald Booms and Tino Leffering for their assistance during laboratory work. This work is part of the Netherlands Organisation for Scientific Research (NWO) research programme "Green Aquafeeds" (project number 870.15.100). DuPont Animal Nutrition partly provided the enzymes, analysed the enzyme recovery and partly funded the project. 


$$
\therefore
$$




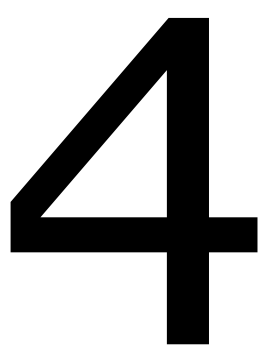

Effect of exogenous enzymes (phytase and xylanase) supplementation on nutrient digestibility and growth performance of Nile tilapia fed different quality diets

Roel M. Maas, Marc C.J. Verdegem, Theodor L. Stevens, Johan W. Schrama

Aquaculture (2020), 529: 735723 
Abstract

The use of plant by-products in aqua-feeds contributes to improving the sustainability of aquaculture, but also leads to increased levels of undesirable nonstarch polysaccharides (NSP) and phytate. NSP-degrading enzymes (i.e. xylanase) and phytase can be used as a tool to deal with NSP and phytate. A feeding trial was conducted to test whether the effects of phytase and xylanase supplementation on growth performance and nutrient digestibility in Nile tilapia are dependent on diet quality. Two diets were formulated, a control quality (CQ diet; $36 \%$ protein and $17 \%$ NSP) and a low quality diet (LQ diet; $32 \%$ protein and $30 \%$ NSP). The difference in diet quality was created by using higher levels of wheat bran, rapeseed meal, sunflower meal, rice bran and wheat dried distiller's grain with solubles in the LQ diet. Both diets had the following enzyme treatments: 1) no enzymes, 2) phytase, and 3) phytase and xylanase. Phytase (Buttiauxella sp.) was supplemented at ca. $660 \mathrm{FTU} / \mathrm{kg}$ and xylanase at ca. $6596 \mathrm{U} / \mathrm{kg}$. In total, 18 tanks (6 treatments, 3 replicates per treatment) were used with 30 fish each (mean initial body weight $39 \mathrm{~g}$ ). Fish were restrictively fed at $80 \%$ of expected satiation for 42 days, by hand twice daily. Growth was determined by batch weighing of the fish at the start and at the end of the trial. Faeces were collected non-invasively using settling units in order to determine the nutrient digestibility, using yttrium as an inert marker. Fish fed the LQ diet had lower growth $(1.35 \mathrm{~g} / \mathrm{d}$ vs. $1.52 \mathrm{~g} / \mathrm{d}$ ) and nutrient digestibility (except for calcium and ash), compared to the CQ diet $(P<0.05)$. Phytase improved the digestibility of dry matter, total carbohydrates, NSP, energy, ash, phosphorus and calcium $(P<0.01)$ Phytase improved growth $(\mathrm{g} / \mathrm{d})$ by $\mathrm{ca} .7 \%$ and phosphorus availability by ca. $29 \%$. The improvement in growth with phytase was comparable between the two diets, improving the FCR from 1.04 to 0.97 and from 1.17 to 1.10 for the CQ and LQ diet, respectively. Xylanase supplementation, on top of phytase, did not enhance growth $(P>0.05)$. Xylanase improved digestibility of dry matter, energy, total carbohydrates and NSP (from $29.7 \%$ to $36.6 \%$ ) of the LQ diet, but not of the CQ diet (interaction; $P<0.05$ ). In conclusion, the effect of phytase on improved nutrient digestibility and performance was independent of diet quality, whereas the effect of xylanase was dependent on diet quality. 


\section{Introduction}

Aquaculture production is increasing in volume and importance. In 2016, aquaculture accounted for $50 \%$ of worldwide fish (including molluscs, crustaceans, seaweeds etc.) production and about $7 \%$ of the protein consumed by humans (FAO, 2018). With the expansion of aquaculture, global production of aqua-feeds is also increasing and aqua-feed formulations now include a range of ingredients that were not previously used for feeding fish and other aquatic animals. For example, increasing cost and limited global production of fish-meal and marine fish oils has led to increased use of terrestrial plant ingredients in aqua-feeds (Gatlin III et al., 2007; Hardy, 2010; NRC, 2011; Tacon and Metian, 2015). Coinciding with this change, the levels of phytate and non-starch polysaccharides (NSP) in aqua-feeds have increased, especially when plant by-products are used as feed ingredients. Such products include wheat bran, rice bran, citrus pulp and wheat DDGS (Dried Distiller's Grains with Solubles) (Choct, 1997; Knudsen, 1997; Sinha et al., 2011). These products are of interest as ingredients in aqua-feeds from a sustainability perspective, and also because they are of little value for human consumption (Troell et al., 2014).

Phytate bound phosphorus $(P)$ is unavailable for fish, as fish lack endogenous phytase to hydrolyse inositol-phosphate linkages. Phytate has a strong binding affinity to cations, such as $\mathrm{Ca}^{2+}, \mathrm{Mg}^{2+}, \mathrm{Zn}^{2+}, \mathrm{Cu}^{2+}, \mathrm{Fe}^{3+}$, and $\mathrm{K}^{+}$, to form insoluble salts. This reduces the bioavailability of minerals. Furthermore, phytate can form insoluble complexes with proteins, thereby reducing protein digestibility (Cao et al., 2007; Kumar et al., 2012; Riche and Garling, 2004). NSP is difficult to digest and can induce a wide range of anti-nutritive effects, related to alterations in digesta viscosity, gut physiology, gut morphology, and gut microbiota composition (Sinha et al., 2011). The lowered nutrient availability induced by dietary phytate and NSP can reduce fish growth (Kumar et al., 2012; Maas et al., 2020a), thus limiting the potential for inclusion of plant ingredients rich in phytate and NSP in aquafeeds. Furthermore, reduced nutrient utilisation increases waste production, increasing the system waste load in recirculating aquaculture systems (RAS) and eutrophication when waste is discharged to surrounding water bodies (Amirkolaie, 2011; Mainstone and Parr, 2002).

Supplementation of diets with NSP-degrading enzymes (i.e., xylanase, $\beta$ glucanase and cellulase) and phytase can (partly) neutralise the negative effects of phytate and NSP on digestion and thereby increase fish growth (Castillo and Gatlin III, 2015, Kumar et al., 2012; Maas et al., 2018). Numerous studies have been performed with phytase supplementation in different fish species, such as rainbow trout (Oncorhynchus mykiss) (Cheng et al., 2004; Sugiura et al., 2001), sea bass (Dicentrarchus labrax) (Oliva-Teles et al., 1998), Nile tilapia (Oreochromis niloticus) (Liebert and Portz, 2005; Portz and Liebert, 2004) and Asian catfish (Pangasius pangasius) (Debnath et al., 2005). Across studies and species, phytase supplementation increases dietary $\mathrm{P}$ availability. In addition, several studies show that phytase improves the availability of other minerals and 
thereby promotes growth (Kumar et al., 2012). Maas et al. (2018) showed that phytase supplementation also improved total carbohydrate digestibility, which was related to an increase in NSP digestibility. The results from studies on NSPdegrading enzymes in fish are less conclusive than those on phytase. Some studies found no or limited effects (Ogunkoya et al., 2006; Yigit and Olmez, 2011), whereas others found improved nutrient digestibility and growth (Ghomi et al., 2012; Lin et al., 2007). Due to the variety in type and dose of enzymes used, individually or in combination, and the large differences in diet composition (e.g. type of NSP), it is difficult to make comparisons across studies with regard to the effect of NSP-degrading enzymes (Adeola and Cowieson, 2011; Castillo and Gatlin III, 2015). In most studies on NSP-degrading enzymes in fish, NSP digestibility has not been measured, although it could help explain the large variations in apparent digestibility coefficients (ADCs) reported for other nutrients. Part of the variability might relate to differences in nutrient and/or ingredient composition of diets, because NSP composition of the diet affects the response to enzyme supplementation in terms of growth and nutrient digestibility (Maas et al., 2019). In broilers, the magnitude of the effect of exogenous enzymes on ileal amino acid digestibility is influenced by the nutritional characteristics of the control diet (Cowieson and Bedford, 2009). It is hypothesised that the effect of enzyme supplementation can be influenced by the quantity of the target substrate, such as phytate or NSP. We expect that when the control diet is of a low quality (i.e. low digestibility) or when anti-nutritional effects are expressed, there is a greater potential for improvement (digestibility and/or improved growth) with enzyme supplementation. Furthermore, the level of the target substrate might influence the optimal dose of the enzyme. Flexibility in dosing of enzyme products is preferred over fixed doses so that the economic viability of the product is optimised (Adeola and Cowieson, 2011; Cowieson and Ravindran, 2008).

Hitherto, there have been no studies carried out to evaluate whether the effect of enzyme supplementation is dependent on the quality of the diet, i.e. the level of dietary NSP and phytate. Therefore, the present study investigated whether the effects of phytase and xylanase supplementation differ when supplemented to diets of different quality. In a feeding trial using a control quality and low quality diet, the effect of phytase, xylanase, and their interactions on nutrient digestibility and growth of Nile tilapia were evaluated.

\section{Materials and methods}

\section{Diets}

The impact of enzyme supplementation on nutrient digestibility and growth in tilapia were measured using control quality (CQ) and low quality (LQ) diets. These diets were supplemented with either phytase $(\mathrm{CQ}+\mathrm{Ph}, \mathrm{LQ}+\mathrm{Ph})$, phytase and xylanase $(\mathrm{CQ}+\mathrm{PhX}, \mathrm{LQ}+\mathrm{PhX})$ or not supplemented $(\mathrm{CQ}+0, \mathrm{LQ}+0)$. The $\mathrm{CQ}$ diet variants were formulated to have moderate levels of wheat DDGS, wheat bran, rapeseed meal, sunflower meal and rice bran (Table 1 ). The LQ variants had higher 
levels of these ingredients and no maize starch or pea and soy protein concentrates (Table 1). This resulted in a higher NSP and phytate content in the LQ+0, LQ+Ph and $\mathrm{LQ}+\mathrm{PhX}$ diets than in the $\mathrm{CQ}+0, \mathrm{CQ}+\mathrm{Ph}$ and $\mathrm{CQ}+\mathrm{PhX}$ diets (Table 2 and Supplementary table (S1)). The diets were formulated to have $5.4 \mathrm{~g}$ of available $\mathrm{P} / \mathrm{kg}$ dry matter (DM) using monocalcium phosphate as the P source. The LQ diets had a lower crude protein content than the CQ diets (ca. $32 \%$ vs ca. $36 \%$ ), but the diets were formulated to have similar digestible protein to digestible energy ratios (S1). Diets supplemented with phytase (Axtra ${ }^{\circledR}$ PHY, Buttiauxella sp. phytase, DuPont Animal Nutrition) had a phytase activity of ca. $660 \mathrm{FTU} / \mathrm{kg} \mathrm{DM}$, and diets with added xylanase (Danisco ${ }^{\circledR}$ Xylanase, DuPont Animal Nutrition) had xylanase activity that was higher than originally intended (6000 U/kg DM) (Table 2). Yttrium oxide was included as the inert marker for digestibility studies.

Extruded diets $3 \mathrm{~mm}$ pellets were produced by Research Diet Services (Wijk bij Duurstede, The Netherlands). The oils (fat) and enzymes were added to the pelleted diets by vacuum coating (Vacuüm core coater, Pegasus ${ }^{\circledR}-10 \mathrm{VC}, 1 / 4 \mathrm{H} / \mathrm{VV}$ nozzle nr. 6502), at the research facilities of the Animal Science Group, Wageningen University, The Netherlands. The enzymes were diluted 1:50 with demineralised water, and 5-ml of diluted enzymes per $\mathrm{kg}$ feed were added to the oils. Diets without enzymes were coated with $5-\mathrm{ml}$ of demineralised water per $\mathrm{kg}$ of feed. Diets were produced ca. 4 weeks prior to the start of the trial and stored in a refrigerator $\left(4^{\circ} \mathrm{C}\right)$ throughout the entire trial.

\section{Fish rearing conditions and housing facilities}

This experiment was evaluated by the Animal Welfare Body of Wageningen University, The Netherlands. All procedures applied to the animals were in line with Dutch legislation (Act on Animal Experiments) and were classified as not being an animal experiment according to Dutch legislation.

The experiment was conducted in the Aquaculture Research Facility of Wageningen University, The Netherlands. Male Nile tilapia (Oreochromis niloticus) of the strain Silver $\mathrm{NMT}^{\mathrm{TM}}$ were obtained from a commercial fish breeder (Til-Aqua international, Someren, The Netherlands) at ca. $0.2 \mathrm{~g}$ individual weight. Sixteen days prior to the start of the trial, fish were held in 120-L tanks (100 fish/tank, $31 \mathrm{~g}$ initial weight) in the same room and under the same conditions (water quality, photoperiod, etc.) as the feeding trial. Fish were fed a commercial feed (ME-1.8 MP Presta, containing 55\% protein and 15\% fat, Skretting, Stavanger, Norway) prior to the start of the trial. Eighteen rectangular $(70 \times 35 \times 40)$ glass tanks of 70-L (60-L effective volume), placed in three rows of six were used in the trial. Each tank was covered with a lid that prevented light from penetrating from the top. The tanks were connected to the same RAS, which resulted in a common water supply with the same water quality for the inflow of each tank. The RAS consisted of a sump, settling tank and trickling filter. Each tank was connected to a swirl separator (AquaOptima AS, column height $44 \mathrm{~cm}$; diameter $24.5 \mathrm{~cm}$ ) with a detachable glass bottle to collect waste feed spills and faeces. The water flow 
Table 1 Ingredient composition of the experimental diets.

\begin{tabular}{|c|c|c|}
\hline Diet & Control Quality (CQ) & Low Quality (LQ) \\
\hline \multicolumn{3}{|l|}{ Ingredients $(\mathrm{g} / \mathrm{kg})$} \\
\hline Maize & 70 & 70 \\
\hline Maize starch & 150 & - \\
\hline Soyabean meal & 100 & 100 \\
\hline Wheat & 59.3 & 58.8 \\
\hline Wheat gluten meal & 120 & 80 \\
\hline Wheat bran & 60 & 125 \\
\hline Rapeseed meal & 50 & 120 \\
\hline Sunflower meal & 50 & 120 \\
\hline Rice bran full fat & 60 & 125 \\
\hline Wheat DGGS ${ }^{+}$ & 50 & 120 \\
\hline Fish oil & 10 & 10 \\
\hline Rapeseed oil & 20 & 15 \\
\hline Palm oil & 20 & 15 \\
\hline Soy protein concentrate & 90 & - \\
\hline Pea protein concentrate & 50 & - \\
\hline Vitamin and mineral premix ${ }^{\ddagger}$ & 10 & 10 \\
\hline Calcium carbonate $\left(\mathrm{CaCO}_{3}\right)$ & 5 & 5 \\
\hline Monocalcium phosphate (MCP) & 14.5 & 13 \\
\hline DL Methionine & 5.5 & 4.5 \\
\hline L-Lysine $\mathrm{HCl}$ & 3.5 & 6.5 \\
\hline L-Threonine & 2.0 & 2.0 \\
\hline Yttrium oxide & 0.2 & 0.2 \\
\hline
\end{tabular}

${ }^{+}$DDGS; dried distillers grains with solubles.

${ }^{\ddagger}$ Premix composition. Vitamins (expressed as IU or $\mathrm{mg} / \mathrm{kg}$ of final diet): vitamin $\mathrm{B} 1,10$; vitamin $\mathrm{B} 2$, 10; vitamin B6, 10; vitamin B5, 10; vitamin B3, 20; biotin, 0.2; B-12, 0.015; folic acid, 2; vitamin C, 100; vitamin E, 100 IU; A-vitamin A palmitate, 3000 IU; D-Rovimix D3-500, $2400 \mathrm{IU}^{2} \mathrm{~K}_{3} \mathrm{~K}$ menadione sodium bisulphite (51\%), 10; inositol, 400; choline, 1500; Antioxidant BHT (E300-321), 100; calcium propionate, 1000 . Minerals (expressed as $\mathrm{mg} / \mathrm{kg}$ of final diet): ferric sulphate, 50 ; zinc sulphate, 30; cobalt sulphate, 0.1 ; copper sulphate, 10; Sodium selenite, 0.5 ; manganese sulphate, 20; magnesium sulphate, 500; chromic chloride, 1 ; calcium iodate, 2.

through each tank was set at $7 \mathrm{~L} / \mathrm{min}$ using a hand held liquid rotameter, and all tanks contained a cylinder shaped air stone to ensure adequate oxygenation. The photoperiod was set to $12 \mathrm{~h}$ light: $12 \mathrm{~h}$ dark (lights on 7:00, lights off 19:00). The light intensity was increased to the intended level over a period of 30 min (start 06:30) and decreased in the evening over a period of $30 \mathrm{~min}$ (start 18:30). The daily water refreshment of the system was 300-L. Water quality parameters were measured three times per week (Monday-Wednesday-Friday), in the morning before feeding to ensure that the water quality parameters remained within the pre-set ranges. Water samples were collected from the common outflow. The average temperature was $27.5{ }^{\circ} \mathrm{C} \pm 0.2$. The $\mathrm{pH}$ range was set between 7.0 and 7.8. Sodium bicarbonate was added if the $\mathrm{pH}$ tended to drop below 7.0. The $\mathrm{pH}$ 
ranged between 6.8 and 7.8 during the trial. Dissolved oxygen levels of the common outflow stayed above $6.2 \mathrm{mg} / \mathrm{L}$. Conductivity was $7800 \mu \mathrm{S} / \mathrm{cm}$ at stocking, which was gradually lowered and kept between 3000-4000 $\mu \mathrm{S} / \mathrm{cm}$ after week one. Total ammonia and nitrite levels of the outflow remained below 0.1 $\mathrm{mg} / \mathrm{L}$ and nitrate below $400 \mathrm{mg} / \mathrm{L}$.

Table 2 Analysed chemical composition and enzyme activity of the experimental diets.

\begin{tabular}{|c|c|c|c|c|c|c|}
\hline \multirow[t]{2}{*}{ Diet } & \multicolumn{3}{|c|}{ Control Quality (CQ) diet } & \multicolumn{3}{|c|}{ Low Quality (LQ) diet } \\
\hline & $\mathrm{CQ}+0$ & $\mathrm{CQ}+\mathrm{Ph}$ & $C Q+P h X$ & $\mathrm{LQ}+0$ & $L Q+P h$ & $\mathrm{LQ}+\mathrm{PhX}$ \\
\hline \multicolumn{7}{|c|}{ Analysed nutrient content (g/kg DM) } \\
\hline Dry matter (DM, g/kg) & 936 & 931 & 933 & 954 & 953 & 948 \\
\hline Crude protein & 353 & 357 & 362 & 317 & 317 & 330 \\
\hline Crude fat & 87 & 87 & 89 & 98 & 87 & 92 \\
\hline Carbohydrates $^{\dagger}$ & 500 & 496 & 490 & 513 & 524 & 509 \\
\hline Starch & 308 & 306 & 302 & 191 & 195 & 190 \\
\hline Sugars ${ }^{\ddagger}$ & 16 & 16 & 16 & 20 & 20 & 20 \\
\hline NSP§ & 176 & 174 & 172 & 302 & 309 & 300 \\
\hline Gross energy (kJ/g DM) & 20.5 & 20.9 & 20.8 & 20.7 & 20.7 & 20.6 \\
\hline Ash & 59 & 59 & 59 & 72 & 72 & 69 \\
\hline Phosphorus & 9.9 & 9.7 & 9.7 & 12.2 & 12.1 & 11.5 \\
\hline Calcium & 7.4 & 7.3 & 7.2 & 7.6 & 7.6 & 7.3 \\
\hline Magnesium & 3.1 & 3.1 & 3.1 & 4.6 & 4.6 & 4.3 \\
\hline$D P / D E^{\Uparrow}$ & 19.4 & 19.1 & 19.5 & 18.8 & 18.6 & 18.7 \\
\hline \multicolumn{7}{|l|}{ Enzyme acivity } \\
\hline Phytase (FTU/kg) & b.d. & 662 & 621 & b.d. & 667 & 667 \\
\hline Xylanase (U/kg) & n.m. & n.m. & 6348 & n.m. & n.m. & 6844 \\
\hline
\end{tabular}

Notes. +0, no enzymes added; +Ph, phytase added; +PhX, phytase and xylanase added; b.d., below detection; n.m., not measured.

${ }^{\dagger}$ The carbohydrate content (on DM basis) was calculated as: 1000 - (ash + crude protein + crude fat).

‡ Sugars calculated as: starch and sugars - starch.

$\S$ Non-starch polysaccharides calculated as: carbohydrates - (starch + sugars).

" DP/DE calculated using the level of digestible (D) protein $(P$, in $\mathrm{mg}$ ) and energy $(E$, in $k J)$ in the diet and the digestibility of both $P$ and $E$ (Table 4).

\section{Experimental procedure}

The trial lasted 42 days. At the start, 30 fish were stocked per tank at random ( 3 rounds of $10 \mathrm{fish}$ ), and subsequently batch weighed (per tank) to determine the total biomass. The mean initial body weight at the start of the trial was $39 \mathrm{~g}$. At the end of the trial all fish were batch weighted (per tank) to determine the final weight and to calculate growth parameters. Fish were starved each time for $24 \mathrm{~h}$ before batch weighing to allow for the gastro-intestinal tract to empty. The six experimental diets were assigned randomly per set of six tanks. This resulted in 3 replicates per treatment. 
The aim within the experiment was to test the effect of the added enzymes and dietary NSP level, on growth and digestibility. Therefore, the fish were fed a restricted equal amount of feed (on DM basis) per tank per day, in order to rule out the effect of feed intake on growth and digestibility. The feeding level was aimed at $16 \mathrm{~g} / \mathrm{kg}^{0.8}$ body weight $(\mathrm{BW}) / \mathrm{d}$, which is about $80 \%$ of the expected satiation level. The daily amount of feed was increased throughout the trial by predicting fish growth and weight, using the average start weight of the fish and an expected feed conversion ratio (FCR) of 1.2. At the first feed, the fish were fed $20 \%$ of the intended feed level, which was increased in increments over 5 days to $100 \%$. This was done to allow for habituation to the diet and to prevent feed spills. The daily feed ration was divided into two equal portions fed at 09:00 and 15:30 $\mathrm{h}$. The fish were hand fed and portion feeding did not exceed $1 \mathrm{~h}$ for all tanks. The fish were fed through a funnel, via a hole in the lid. Fifteen min after feeding, the settling bottles $(250-\mathrm{ml})$ connected to the swirl separators were emptied and checked for uneaten pellets. The equivalent amount of uneaten pellets was added to the next feed, to ensure all fish received the same amount of feed ( $\mathrm{g} D \mathrm{DM} / \mathrm{fish}$ ). The feed was sieved to remove small particles and dust, to ensure all feed was consumed and to prevent deterioration of the water quality. Feed was stored in plastic buckets in a fridge at $4{ }^{\circ} \mathrm{C}$, throughout the trial. Each week a $100 \mathrm{~g}$ feed sample of each diet was taken for composition analysis. Each diet sample was added to a specific diet container, which was stored in the fridge at $4{ }^{\circ} \mathrm{C}$, until analysis at the end of the trial.

Faeces were collected for digestibility measurements using a swirl separator, with a detachable set of glass bottle $(500-\mathrm{ml})$. Faeces collection was carried out from the second week onwards. Faeces were collected between the afternoon and morning feeds (between 16:30 - 08:00 h) and the glass collection bottles were submerged in ice to prevent bacterial degradation of the faeces, and placed in Styrofoam boxes overnight for insulation. Faeces were pooled per week (per tank) and stored in aluminium trays at $-20{ }^{\circ} \mathrm{C}$ until further analysis.

\section{Analyses}

Feed and faeces were analysed as described by Staessen et al. (2020). Batches of faeces collected per week per tank were dried at $70{ }^{\circ} \mathrm{C}$. The dry weight collected during the last two weeks (pooled) was sufficient to perform all the analyses, hence faeces collected earlier were not used. The faeces were ground using a mixer mill (Retsch Brinkmann, model MM2000) prior to the analysis. Collected faeces and feed were analysed gravimetrically for DM by drying at $103^{\circ} \mathrm{C}$ for $4 \mathrm{~h}$ until constant weight. Following the DM determination, ash content was determined gravimetrically by incineration in a muffle furnace for $4 \mathrm{~h}$ at $550{ }^{\circ} \mathrm{C}$ (ISO 5984, 1978). Ashed samples were transferred to volumetric flasks and dissolved in concentrated sulphuric acid solution by autoclaving. Samples were subsequently diluted in water and filtered using a syringe filter (45 $\mu \mathrm{m}$ pores). Finally, yttrium, $\mathrm{P}$, calcium ( $\mathrm{Ca}$ ) and magnesium $(\mathrm{Mg})$ were analysed using inductively coupled plasma-mass spectrometry (ICP-OES) according to the standard NEN 15510 
(2007). The total nitrogen content was measured using the Kjeldahl-method (ISO 5983 , 1997), calculating crude protein as $\mathrm{N} \times 6.25$ (protein conversion factor). Crude fat was measured gravimetrically using acid hydrolysis followed by petroleum-ether extraction (Soxhlet method; ISO 6492, 1999). Gross energy was measured using a bomb calorimeter (IKA ${ }^{\circledR}$ werke, C7000; IKA analysentechnik, Weitershem, Germany). Starch in feed and faeces was determined enzymatically (NutriControl, The Netherlands). Starch was determined after washing away free sugars with $40 \%$ ethanol. The dried residue was digested with Termamyl ${ }^{\circledR}$, after which starch was hydrolysed using amyloglucosidase. The glucose units formed were determined using the Luff-Schoorl reagent. Starch + sugars was measured as described above, leaving out the washing with $40 \%$ ethanol.

Danisco Animal Nutrition Laboratories (Brabrand, Denmark) analysed the feed samples for phytase activity as described by Yu et al. (2012) and for xylanase activity as described by Romero et al. (2013). For phytase activity, a standard phytase assay method was used (Engelen et al., 2001). Feed samples were reacted with phytic acid at $37{ }^{\circ} \mathrm{C}$ and $\mathrm{pH} 5.5$ for $60 \mathrm{~min}$. The reaction was stopped by adding acid vanadate-molybdate reagent, which produces a coloured complex. The optimal density was monitored at $415 \mathrm{~nm}$. One xylanase unit was defined as the amount of enzyme that releases $0.48 \mu \mathrm{mol}$ of reducing sugar as xylose from wheat arabino xylan per minute at $\mathrm{pH} 4.2$ and $50{ }^{\circ} \mathrm{C}$. Azurine cross linked arabinoxylan isolated from wheat (Megazyme International Ireland Ltd., Bray, Ireland) was used as substrate. Sample extracts $(100 \mu / \mathrm{L})$ were incubated at $50{ }^{\circ} \mathrm{C}$ for $60 \mathrm{~min}$, mixed with a vortex and centrifuged at $960 \times g$ for 10 min, after which absorbance of the supernatant was measured with a spectrophotometer at $590 \mathrm{~nm}$ against a blank sample and units were calculated in reference to a calibration.

\section{Performance}

Growth performance was calculated as described by Saravanan et al. (2012). The growth (in $\mathrm{g} / \mathrm{d}$ ) was calculated as the difference between the average individual initial $\left(\mathrm{W}_{\mathrm{i}}\right)$ and final $\left(\mathrm{W}_{\mathrm{f}}\right)$ body weight per tank, divided by the duration $(\mathrm{t})$ of the trial in days (d). The specific growth rate (SGR in \% BW/d) was calculated as (In $\left.\left(W_{f}\right)-\ln \left(W_{i}\right)\right) \times 100 / t$. The daily absolute feed intake (in g DM/d) was calculated as $\mathrm{FI}_{\text {tot }} /(\mathrm{n} \times \mathrm{t})$, where $\mathrm{n}$ is the number of fish per tank and $\mathrm{FI}_{\text {tot }}$ the total feed intake (in g DM), corrected for mortality and feed spills. The FCR was calculated as daily absolute feed intake / growth. The feed intake expressed as percentage of BW $(\% / d)$ was calculated as $\left(\mathrm{FI}_{\text {tot }} / \mathrm{W}_{\mathrm{G}}\right) \times(100 / \mathrm{t})$, where $\mathrm{W}_{\mathrm{G}}$ is the geometric mean body weight (in $g$ ) calculated as $\sqrt{ }\left(W_{i} \times W_{f}\right)$. The survival of fish per tank was calculated as $\left(\mathrm{N}_{\mathrm{f}} / \mathrm{N}_{\mathrm{i}}\right) \times 100$, where $\mathrm{N}_{\mathrm{f}}$ is the final number of fish and $\mathrm{N}_{\mathrm{i}}$ the initial number. The protein efficiency ratio (PER) was calculated as growth / protein intake, where the protein intake (in $\mathrm{g} / \mathrm{d}$ ) was calculated as $\mathrm{FI}_{\text {tot }} \times$ ( $\%$ protein diet / 100). 


\section{Digestibility}

Yttrium was used as an inert marker to calculate the apparent digestibility coefficient (ADC in \%) of dry matter, crude protein, crude fat, total carbohydrate, starch, NSP, gross energy, ash, $\mathrm{P}, \mathrm{Ca}$ and $\mathrm{Mg}$ for each tank. The ADC was calculated as (Cheng and Hardy, 2002): ADC (\%) $=100 \times\left[1-\left(Y_{i} \times\right.\right.$ amount nutrient in faeces) / $\left(Y_{f} X\right.$ amount nutrient in feed)], where $Y$ is the concentration of yttrium in the diet and faeces and $\mathrm{N}$ is the quantity of nutrient or energy content in the diet and faeces. The total amount of carbohydrates in feed and faeces was calculated on a DM basis as: 1000 - (crude protein + crude fat + ash). The NSP fraction was calculated as: total carbohydrates - (starch + sugars). As the total carbohydrates and NSP fraction were calculated (as above), the means incorporated the accumulated errors in all the other analyses, leading to increased error (SEM). Therefore, this data must be interpreted with care.

\section{Statistical analyses}

The main effect of diet (quality) was tested according to a 2 by 3 factorial design using a General Linear Model (GLM), where the first factor is the diet (CQ vs LQ) and the second factor the enzyme treatment; 1) no enzymes, 2) phytase, and 3) phytase and xylanase. This means that when the CQ or LQ diet is mentioned without enzyme treatment, reference is made to the average of diets based on CQ or LQ, respectively. This 2 by 3 factorial design was also used to calculate the standard error of the mean (SEM) presented in Tables 3 and 4. The effect of phytase and the interaction between diet and phytase was tested according to a two-way ANOVA on part of the data set, using both diets with no enzymes and with only phytase supplementation, thus excluding the diets with the combined phytase and xylanase supplementation. Likewise, the effect of xylanase and the interaction between diet and xylanase was tested according to a two-way ANOVA on part of the data set, using both diets supplemented with phytase and the combined supplementation with phytase and xylanase, thus excluding the diets with no enzymes. When an interaction was found $(P<0.05)$ between diet and phytase and/or xylanase, a Tukey HSD (honest significant difference) with multiple comparison with $95 \%$ level of significance was used to compare treatment means. All data were expressed as the mean per treatment of three replicates. All statistical analyses were carried out using the IBM Statistical Package for the Social Sciences (SPSS) program ( $v$ 25.0, New York, United States). 


\section{Results}

\section{Growth}

The average initial weight was $39.3 \mathrm{~g}$ and statistically identical for all treatments $(P>0.1)$. The overall survival rate within the trial was high at $99.4 \%$ and unaffected by dietary treatment (Table 3). As intended, fish on different dietary treatments received equal amounts of feed $(1.50 \mathrm{~g} \mathrm{DM} / \mathrm{d})$. There was a dietary effect on all performance parameters $(P<0.05)$. The lower quality of the LQ diet lowered the growth rate and SGR and increased the FCR. Phytase supplementation improved the growth rate and lowered the FCR (Table $3 ; P<0.001$ ). The improvement in growth with phytase was comparable between the two diets, improving the FCR from 1.04 to 0.97 and from 1.17 to 1.10 for the CQ and LQ diet, respectively. In contrast to the effect of phytase, xylanase did not affect any of the parameters $(P>0.1)$. Numerically, a slightly better FCR, SGR, growth rate $(\mathrm{g} / \mathrm{d})$ and final body weight was observed for the LQ diet with xylanase, while those parameters were highly comparable for the CQ diet. The PER averaged 2.81 for all treatments, and was not influenced by diet quality $(P>0.1)$. Phytase supplementation improved the PER $(P<0.001)$, whereas xylanase supplementation decreased the PER $(P<0.05)$. No interactions between the diet quality and phytase or xylanase were present $(P>0.1)$.

\section{Digestibility}

The low quality of the LQ diet lowered the digestibility of all nutrients $(P<0.05)$ except $\mathrm{Ca}$ and ash (Table 4). The DM digestibility was on average $79.6 \%$ for the CQ diet versus $70.4 \%$ for the LQ diet. As expected, phytase supplementation improvement the digestibility of the ash fraction and the minerals $\mathrm{P}, \mathrm{Ca}$ and $\mathrm{Mg}$ $(P<0.001)$. The $\mathrm{P}$ digestibility improved from $51.4 \%$ to $65.3 \%$ for the CQ diet and from $45.0 \%$ to $58.8 \%$ for the LQ diet. In addition, phytase supplementation resulted in a higher digestibility of DM, carbohydrates and energy $(P<0.01)$. There was an effect of xylanase supplementation as well as an interaction effect between xylanase and the diet for $\mathrm{DM}$, carbohydrate and energy digestibility $(P<0.05)$. For DM, carbohydrate and energy, the digestibility was significantly higher for the LQ diet with xylanase, compared to the LQ diet without xylanase, while the CQ diet did not benefit from the supplementation of xylanase. Without enzyme supplementation, $17.9 \%$ and $25.3 \%$ of the NSP was digested for the CQ and LQ diet, respectively. Phytase improved the NSP ADC from $17.9 \%$ to $22.5 \%$ for the CQ diet and from $25.3 \%$ to $29.7 \%$ for the LQ diet $(P<0.01)$. The improvement in NSP digestibility with xylanase supplementation depended on the diet as shown by the interaction effect $(P<0.01)$. Supplementing xylanase did not lead to a further increase in NSP ADC for the CQ diet, while the NSP ADC was significantly higher with xylanase supplementation (29.7\% vs. $36.6 \%)$ for the LQ diet. 


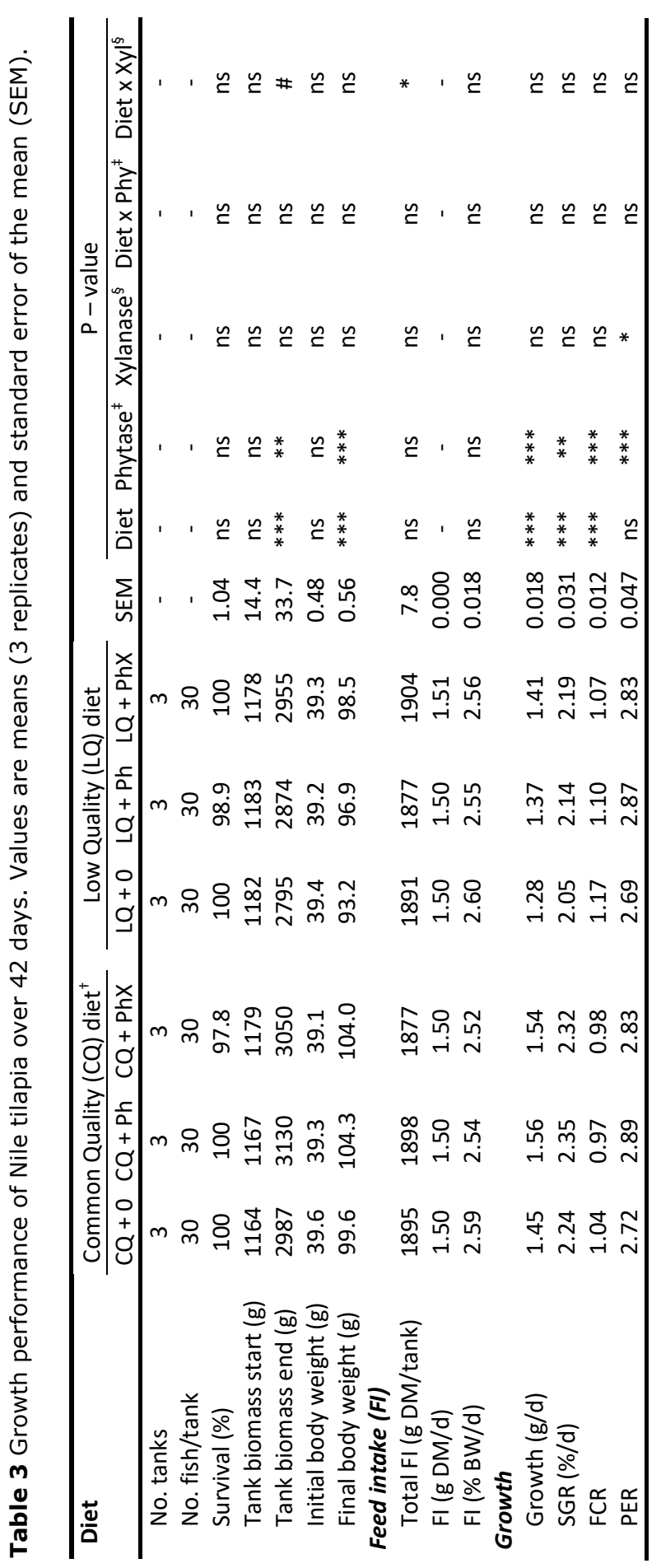

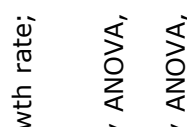

ज़ ते ते

嵌

ज

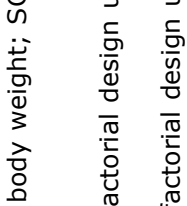

गं

ㅇํㅇ $\mathrm{a}$ ก

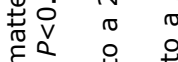

>*

范范

īं 0

苛荡莺

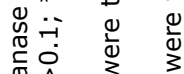

元余方方

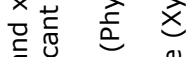

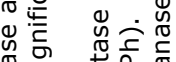

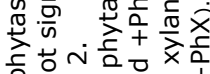
음 응ㅁำ 可 +

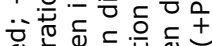

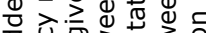

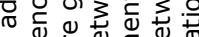

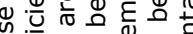

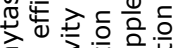

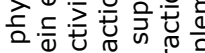
二

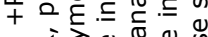

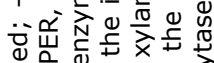

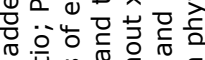

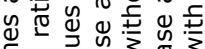

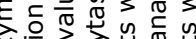

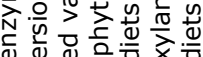

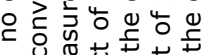
$+\quad$ 过 幽七。

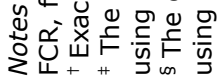




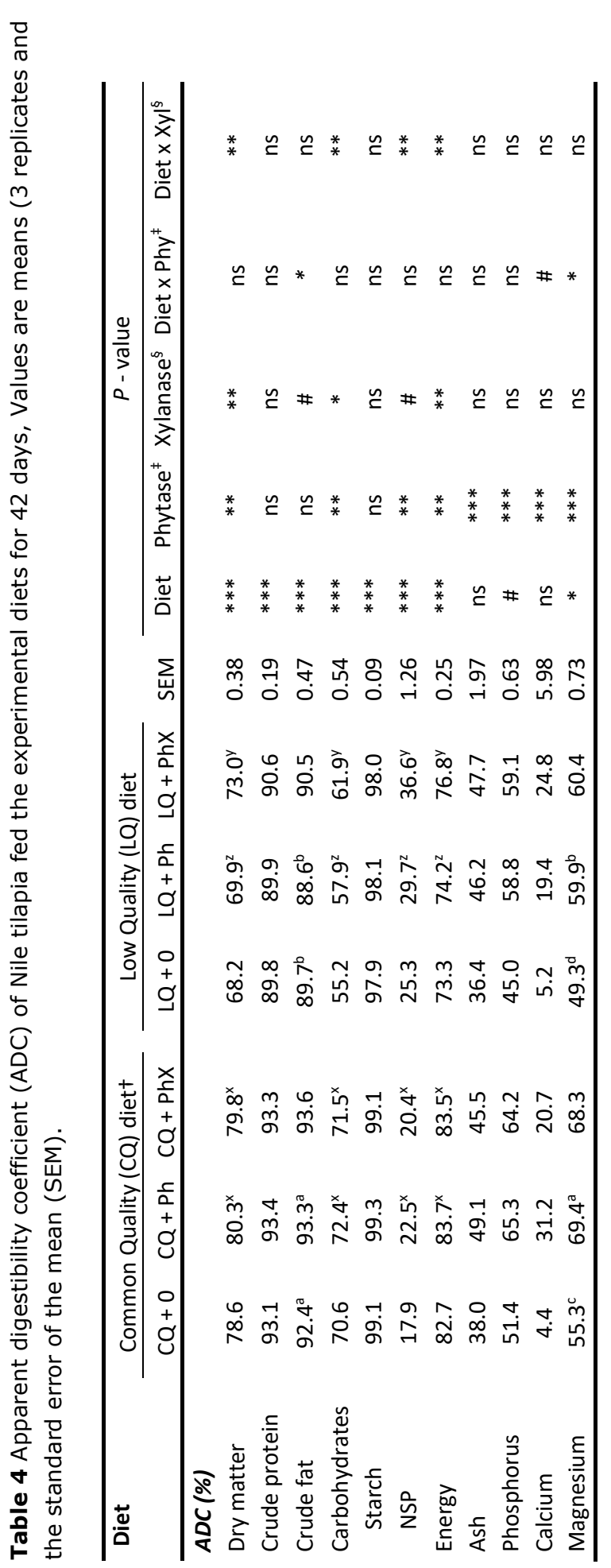

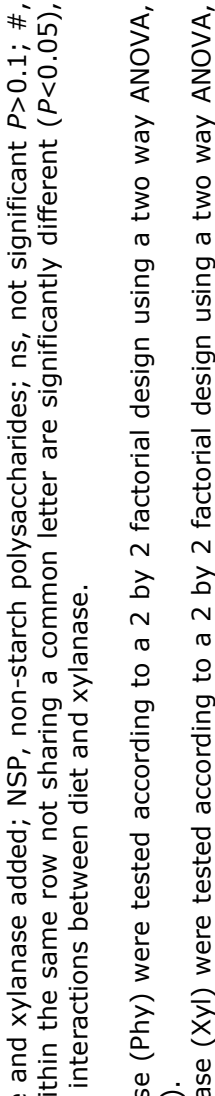

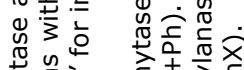

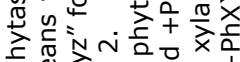

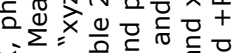

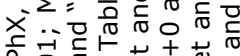
$+8 \begin{aligned} & 0 \\ & +\end{aligned}$

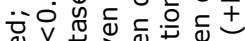

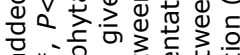
* *

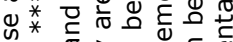

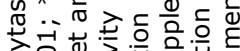

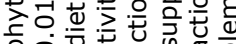
잉 ᄃำ $+*-\sum^{3} \xi . \subseteq \frac{c}{\sigma}=$

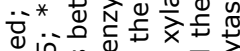

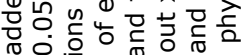
o 0

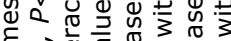

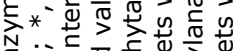
N OVं † 方兑

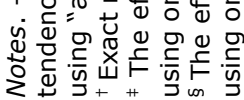




\section{Discussion}

\section{Diet quality}

The experiment investigated whether the effect of phytase and xylanase supplementation on nutrient digestibility and growth depends on the quality of the diet. Two diets were used, a control quality (CQ) diet and a low quality diet (LQ), with a NSP content of 174 and $304 \mathrm{~g} / \mathrm{kg}$ DM respectively. The difference in diet quality is a combination of factors, besides the difference in NSP content, the LQ diet had higher phytate levels (S1), lower protein levels (Table 2) and a different protein source composition (Table 1 ). The lower quality of the LQ diet reduced all growth performance parameters, which is in line with previous studies with Nile tilapia using diets with a contrast in NSP level (Haidar et al., 2016; Maas et al., 2019). As a consequence of the lower quality (NSP level), the digestibility of the carbohydrate fraction was reduced from an ADC of $70.6 \%$ for the CQ diet to $55.2 \%$ for the LQ diet without enzymes. This study shows that, besides these direct effects of lower diet quality (increased NSP level), the protein, fat, ash, P and Mg digestibility was reduced as well. NSP can have multiple anti-nutritional factors, such as the deconjugation of bile acids (which are important for fat digestion), affecting the gut morphology (i.e., shortening of villi length) and affecting the digesta viscosity which can reduce the mixing of digestive enzymes. All these factors can contribute to reduced nutrient digestibility (Choct, 1997; Choct and Kocher, 2000; Sinha et al., 2011). Furthermore, soy and pea protein concentrates were used (14\% in total) in the CQ diet, whereas all the protein in the LQ diet originated from low quality ingredients, which elevated the level of, amongst others, dietary NSP. Likewise, the percentage of ash and minerals that originated from the mineral premix was higher for the CQ diet. The choice of ingredients is a factor that influences the quality of the diet, which in turn is known to influence the overall nutrient $A D C$, irrespective of the nutrient composition (Alonso et al., 2000; Cheng and Hardy, 2003).

NSP was shown not to be inert for endogenous digestion, which is in line with several studies using Nile tilapia (Amirkolaie et al., 2005; Haidar et al., 2016; Leenhouwers et al., 2007a; Maas et al., 2019). In the present study, an endogenous (without enzyme supplementation) NSP digestibility of $17.9 \%$ and $25.3 \%$ was found for the CQ and LQ diet, respectively. The ADC values are comparable to the average calculated NSP digestibility of $24.3 \%$ for tilapia across studies in a recent review by Maas et al. (2020a). The NSP source and composition is a known factor influencing the endogenous NSP digestibility (Leenhouwers et al., 2007a; Maas et al., 2019). However, in the present study, the theoretical NSP composition was kept comparable, by incorporating the NSP rich ingredients (wheat bran, rapeseed meal, sunflower meal, rice bran, and wheat DGGS) in a similar ratio into the diets. With the used NSP levels in the diets (Table 2) and the NSP ADCs (Table 4), it was calculated that approximately $31 \mathrm{~g}$ versus $77 \mathrm{~g}$ of NSP was digested endogenously per $\mathrm{kg}$ DM feed fed, for the CQ and LQ diets, respectively. On the contrary, Haider et al. (2016) showed that the NSP 
digestibility of the same diet drastically decreased with an increase in feeding level, which suggest a threshold in the amount of NSP that can be digested. It is unclear what caused the higher NSP ADC, and a more than two-fold increase in absolute NSP digestion, for the LQ diet compared to the CQ diet.

\section{Phytase}

Supplementation of microbial phytase in the diet improved $\mathrm{P}$ availability and overall growth performance, while reducing $P$ excretion for several fish species (Cao et al., 2007; Cheng et al., 2004; Kumar et al., 2012; Oliva-Teles et al., 1998). Hereby, the induced anti-nutritional effect of phytate was partly compensated, reducing the need for inorganic $P$ (Cao et al., 2007). In line with other studies on Nile tilapia (Liebert and Portz, 2005; Maas et al., 2018; Portz and Liebert, 2004), the growth performance was improved for both diets with phytase supplementation. The available P level (in $\mathrm{g} / \mathrm{kg}$ DM feed) was 5.1 for the CQ diet and 5.4 for the LQ diet, well above the stated $P$ requirement across species for growth of $4.7 \mathrm{~g} / \mathrm{kg}$ DM feed (Prabhu et al., 2013). In line with a comparable improvement in growth, the improvement in $\mathrm{P}$ digestibility was comparable between the $\mathrm{CQ}$ and $\mathrm{LQ}$ diets. The large and comparable impact phytase has on both growth performance and $P$ digestibility for the CQ and LQ diets suggests that $P$ availability was limited for growth, which suggests that the stated available $P$ level of 4.5 ( $\mathrm{g} / \mathrm{kg} \mathrm{DM}$ feed) is too low to support optimal growth. In the present study, an enzyme dose of $660 \pm 24 \mathrm{FTU} / \mathrm{kg}$ diet was measured (aimed for 1000 $\mathrm{FTU} / \mathrm{kg}$ diet). The enzyme dose used was below the optimal range reported for tilapia by Liebert and Portz (2005) of 750-1000 FTU/kg. The increase in P digestibility is highly comparable between both diets, while the level of $P$ in the diet is approximately $23 \%$ higher for the LQ diet. From the P level in the diet (Table 2) and the P ADC (Table 4), it was calculated that 1.36 and $1.67 \mathrm{~g} \mathrm{P}$ per $\mathrm{kg}$ DM feed administered, was made available with phytase, for the CQ and LQ diets, respectively. This suggest that the dose of phytase $(660 \pm 24 \mathrm{FTU} / \mathrm{kg})$ was not limiting for increasing the $P$ availability of both diets (see section "Xylanase" below). The slightly higher P digestibility for the CQ diet compared to the LQ diet, both with and without enzymes, can be attributed to the higher percentage of highly digestible inorganic $P$ (monocalcium phosphate) in the total $P$ content in the CQ diet. The effect phytate and phytase have on, in particular, the bio-availability of minerals and the amino acid digestibility, is well described (Cao et al., 2007; Francis et al., 2001; Nolan et al., 1987). To our knowledge, the effect of phytase on improving NSP digestibility has not been reported before. Maas et al. (2018) showed a strong increase in carbohydrate digestibility with phytase supplementation, and suggested that this was due to improved NSP digestibility. NSP are structural components of plants which primarily can be found in cell walls (Sinha et al., 2011). Where phytate, the main storage form of $P$ in plants, is found within the plant largely depends on the type of plant. In cereals for example, phytate is mainly concentrated in the aleurone layer, whereas in legumes, phytate is distributed throughout the whole seed (Skoglund et al., 2009). It can be hypothesised that when, for example, the phytate in the aleurone layer is broken 
down through phytase supplementation, the NSP, located in the aleurone layer becomes more accessible for endogenous digestion, with increased NSP digestibility as result.

\section{Xylanase}

The effect of xylanase was tested in diets that were all supplemented with phytase. In our study, xylanase did not further enhance growth performance. Studies that have tested the sole effect of xylanase in fish are scarce. A few studies have tested the main effect of xylanase and showed an improvement in growth performance in Nile tilapia (Hassaan et al., 2019), rainbow trout (Dalsgaard et al., 2012), Jian carp (Cyprinus carpio var. Jian) (Jiang et al., 2014) and Japanese seabass (Lateolabrax japonicus) (Ai et al., 2007). All these studies were done without phytase supplementation to the diet. In poultry it is shown that the magnitude of the effect of enzyme supplementation on ileal amino acid digestibility is largely dependent on the nutritional value of the control diet. It is suggested that the effect of carbohydrases (like xylanase) is less when the diet already contains phytase, because phytase can improve the diet's nutritional value compared to a control diet without enzymes (Cowieson and Bedford, 2009). Likewise, in tilapia it is suggested that a control diet low in NSP and phytate, which results in good growth performance, has little to gain from the supplementation of enzymes (Maas et al., 2019). Similarly, the supplementation of phytase in the present study, could have maximised the growth potential of the diet, muting the effect of xylanase. On the contrary, Maas et al. (2018) showed that in Nile tilapia, the effect of xylanase on growth performance was enhanced in the presence of phytase. Although not improving growth rate, xylanase improved the digestibility of DM, total carbohydrates and energy. An interaction effect was present for DM, total carbohydrates, NSP and energy; the digestibility was significantly higher for the LQ diet with xylanase supplementation, while the CQ diet did not benefit (Table 4). Although not statistically significant, both growth rate $(\mathrm{g} / \mathrm{d})$ and FCR were improved by approximately $3 \%$ when xylanase was supplemented to the LQ diet. Although the NSP digestibility with only phytase supplementation to the LQ diet was already higher compared to the CQ diet (22.5\% vs. $29.7 \%$ ), the digestibility increased from $29.7 \%$ to $36.6 \%$, whereas the NSP digestibility of CQ diet did not improve. As mentioned before, because the composition of the NSP fraction of the diets is theoretically comparable, a comparable response was expected. If the enzyme dose was limiting for NSP degradation, a lower response for the LQ diet would be expected, because for each $1 \%$ increase (absolute) in NSP ADC, 75\% more NSP was digested in the LQ diet than in the CQ diet. The NSP digestibility of the LQ diet is higher compared to the CQ diet when supplemented with both phytase and xylanase $(20.4 \%$ vs $36.6 \%)$, which resulted in three times more NSP digested per $\mathrm{kg}$ diet fed, than for the CQ diet (35 vs. $110 \mathrm{~g} / \mathrm{kg}$ diet). Why a larger NSP fraction remained undigested in the $C Q$ diet remains unclear. The effect of xylanase on NSP digestibility might have been partially muted by the endogenous NSP digestibility as well as by the effect of phytase on NSP digestibility, considering that both work on the same NSP fraction. For xylanase it is evident that it cleaves 
the $\beta 1,4$ backbone of xylan, which is part of the hemicellulose fraction (Collins et al., 2005). It is generally accepted that the NSP fractions pectin and hemicellulose are less inert to digestion compared to cellulose and lignin (Amirkolaie et al., 2005; Maas et al., 2019; Sinha et al., 2011). Which part of the NSP fraction is digested endogenously and which part is digested as a result of phytase, remains unclear. With the improvement of DM digestibility, the amount of fecal waste $(100 \%$ - DM ADC) decreased by approximately $10 \%$, reducing the pressure on RAS purification systems or decreasing the nutrient load in the effluent when discharged to surface waters.

\section{Conclusion}

Our study confirmed the negative effect of poor quality diet (high NSP levels, etc.), formulated using low quality ingredients, on growth performance and on digestibility of DM, total carbohydrates, NSP, energy, ash and the minerals $\mathrm{P}, \mathrm{Ca}$ and $\mathrm{Mg}$ in Nile tilapia. Phytase improved the growth performance and nutrient digestibility of DM, energy, total carbohydrates, ash, P and Ca independently of the quality of the diet. Xylanase supplementation on top of phytase did not enhance growth performance of neither the control quality nor the low quality diet. The effect of xylanase on nutrient digestibility depended on the diet quality; xylanase enhanced the digestibility of DM, energy, total carbohydrates and NSP of the low quality diet, while the control quality diet remained unaffected.

\section{Acknowledgements}

We thank the staff of the aquaculture research facilities for their technical support in conducting the experiment and Ronald Booms and Tino Leffering for their assistance during laboratory work. This work is part of the Netherlands Organisation for Scientific Research (NWO) research programme "Green Aquafeeds" (project number 870.15.100). DuPont Animal Nutrition partly provided the enzymes, analysed the enzyme recovery and partly funded the project. 


\section{Supplementary information}

Table S1 Calculated nutrient composition of the experimental diets.

\begin{tabular}{|c|c|c|}
\hline Diet & Control Quality (CQ) & Low Quality (LQ) \\
\hline \multicolumn{3}{|c|}{ Calculated nutrient content ( $\mathrm{g} / \mathrm{kg}$ DM) } \\
\hline Crude protein & 362 & 318 \\
\hline Crude fat & 97 & 107 \\
\hline Carbohydrates & 478 & 495 \\
\hline Energy (GE, kJ/g DM) & 20.9 & 20.2 \\
\hline Non-starch polysaccharides & 168 & 278 \\
\hline Starch & 285 & 179 \\
\hline Sugars & 30 & 48 \\
\hline Crude ash & 63 & 80 \\
\hline Phosphorus & 9.5 & 11.2 \\
\hline Available phosphorus & 5.4 & 5.4 \\
\hline Inositol phosphate & 4.2 & 6.1 \\
\hline Calcium & 6.4 & 6.7 \\
\hline $\mathrm{DP} / \mathrm{DE}^{+}$ & 21.5 & 21.7 \\
\hline \multicolumn{3}{|l|}{ Amino Acids in $\mathrm{g} / \mathrm{kg}$ protein } \\
\hline Lysine & 51.5 & 52.1 \\
\hline Methionine & 31.5 & 31.2 \\
\hline Cystine & 16.8 & 16.9 \\
\hline Threonine & 38.9 & 37.1 \\
\hline Tryptophan & 10.4 & 10.0 \\
\hline Isoleucine & 39.9 & 33.9 \\
\hline Leucine & 70.5 & 60.9 \\
\hline Valine & 44.3 & 40.4 \\
\hline Phenylalanine & 47.8 & 40.9 \\
\hline Arginine & 58.9 & 53.6 \\
\hline Tyrosine & 31.1 & 27.4 \\
\hline
\end{tabular}

${ }^{+} \mathrm{DP} / \mathrm{DE}$, ratio between digestible protein ( $\mathrm{DP}$, in $\mathrm{mg}$ ) and digestible energy ( $\mathrm{DE}$, in $\mathrm{kJ}$ ). 

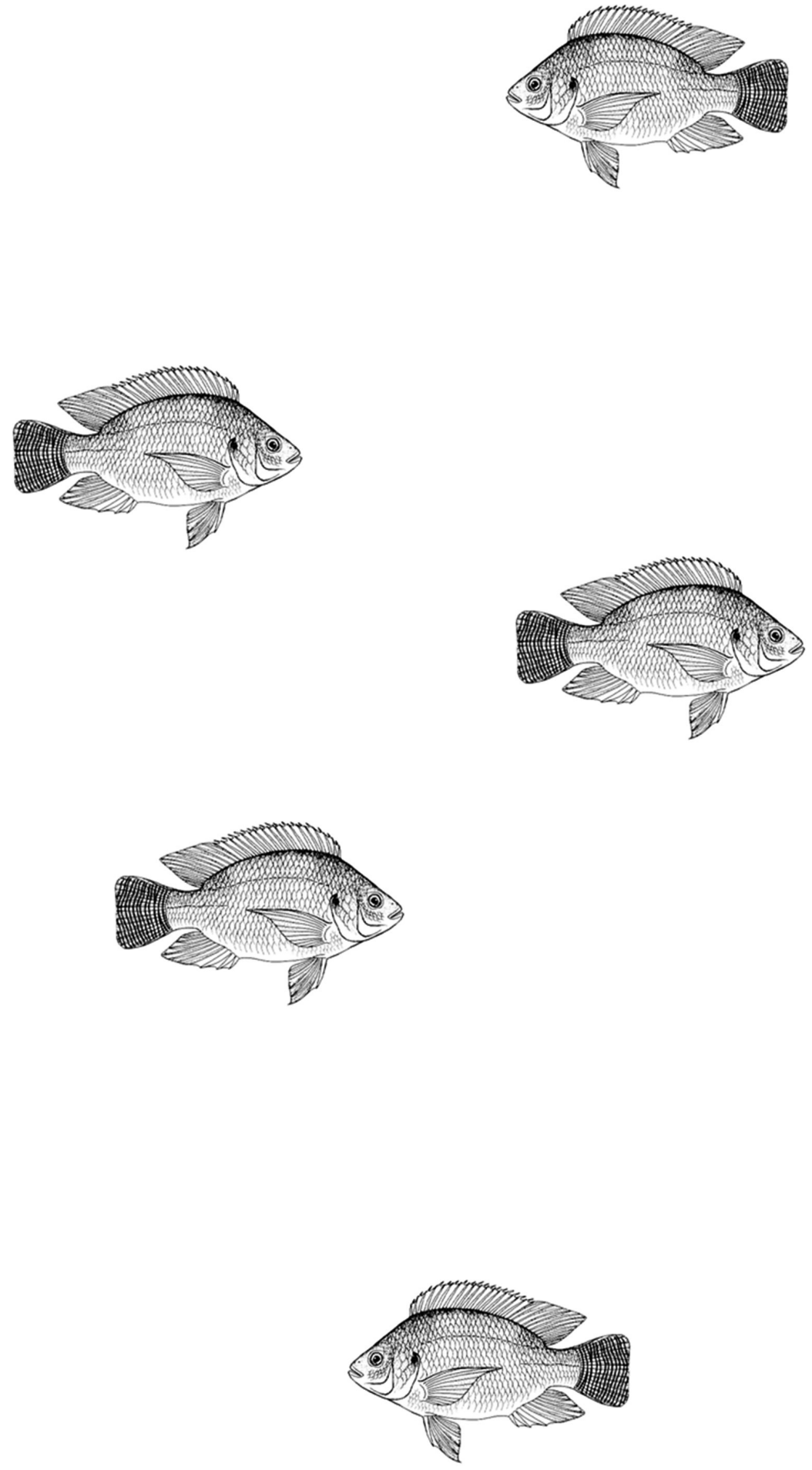


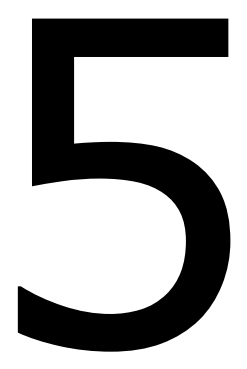

\section{The effect of phytase, xylanase and their combination on growth performance and nutrient utilisation in Nile tilapia}

Roel M. Maas, Marc C.J. Verdegem, Yueming Dersjant-Li, Johan W. Schrama

Aquaculture (2018), 487: 7-14 


\section{Abstract}

Increasing the inclusion rate of plant ingredients will increase the content of nonstarch polysaccharides (NSP) and phytate in the fish feed. Both NSP and phytate are undesired in fish feed due to their anti-nutritional properties. The main objective of the present study was to assess the impact of exogenous enzyme supplementation on growth, body composition, digestibility and the energy, nitrogen and phosphorus balances in Nile tilapia. Four experimental diets were tested in a $2 \times 2$ factorial arrangement of treatments. The first factor was phytase supplementation at a dose of either 0 or $1000 \mathrm{FTU} / \mathrm{kg}$ and the second factor was xylanase supplementation at a dose of 0 or $4000 \mathrm{U} / \mathrm{kg}$. This resulted in a control diet (CON-CON) without enzymes, phytase diet (PHY-CON), xylanase diet (CON$\mathrm{XYL}$ ) and a diet with both xylanase and phytase (PHY-XYL). In total 24 tanks (6 replicates/treatment) were used with 30 (mean initial body weight $42 \mathrm{~g}$ ) fish each. Fish were restrictively ( $80 \%$ of expected satiation) fed the experimental diets for 38 days. Growth was significantly affected by the interaction between phytase and xylanase supplementation $(P<0.05)$, showing a synergism between both enzymes. Growth at the CON-CON and CON-XYL diets were similar, whereas fish fed the PHY-CON had an improved growth. The effect of phytase supplementation on growth was further enhanced when xyalanse was supplemented (PHY-XYL diet). Phytase signicantly improved the digestibility of dry matter, crude protein, carbohydrates, energy, ash, phosphorus and calcium $(P<0.001)$. Xylanase enhanced the digestibility of dry matter, crude protein, carbohydrates and energy significantly $(P<0.05)$. In contrast to growth, there was no significant synergetic effect of the combination of phytase and xylanse on the digestibility $(P>0.05)$. The significant synergetic effect of the combination of phytase and xylanse on growth was not reflected on the digestibility $(P>0.05)$. The nitrogen balance showed that the synergism on growth was predominantly due the significant synergistic effect of phytase and xylanse on the protein retention $(P=0.005)$. Both xylanase and phytase showed to be an effective tool to improve the nutrient availability and growth in Nile tilapia. Fish fed the diet supplemented with both phytase and xylanase had a significantly higher growth than all other treatments. 


\section{Introduction}

The expected future growth of the aquaculture sector increases the pressure of using more sustainable and novel feed ingredients in aqua feeds (Tacon and Metian, 2015). Plant ingredients are nowadays increasingly used to replace fishmeal to reduce cost, to improve the sustainability and to keep up with the demand of high quality protein. This results in a steady decline of dietary fish meal inclusion levels in aquafeeds (Carter and Hauler, 2000; Shepherd and Jackson, 2013). However, with the inclusion of plant ingredients such as soybean and rapeseed meal, the content of non-starch polysaccharides (NSP) as well as phytate will increase in the fish feed. Both NSP and phytate are undesired in fish diets through their anti-nutritional properties (Choct, 1997; Francis et al., 2001; Sinha et al., 2011).

The NSP fraction generally remains undigested, as the enzymes to hydrolyse the glycosidic bonds are scarce or non-existing in the gastrointestinal tract of fish (Kuz'mina, 1996; Choct, 1997; Stone et al., 2003; Sinha et al., 2011). Besides that, the NSP fraction may influence the gut morphology, physiology and mucus layer, affecting the endogenous secretion of water, proteins, electrolytes and lipids. These changes can lead to a reduced nutrient digestibility (Choct, 1997; Sinha et al., 2011). Phosphorus (P) in fishmeal is highly available for growth and metabolism compared to $P$ from plants. In plants $P$ is mostly present in the form of phytate, which is poorly available (Francis et al., 2001; Lall and Hardy, 2003; Lall and Lewis-McCrea, 2007). Increasing levels of dietary phytate reduced the performance of fish (Spinelli et al., 1983; Richardson et al., 1985; Francis et al., 2001). The main effects of phytate are through reduced bio-availability of minerals like $\mathrm{Fe}, \mathrm{Zn}, \mathrm{Mg}, \mathrm{Cu}, \mathrm{Ca}$ and $\mathrm{P}$ and the formation of phytic acid-protein complexes, reducing the solubility of protein (Nolan et al., 1987; Francis et al., 2001) and leading to increased endogenous amino acid losses. Moreover, a low P availability leads to an increasing discharge of $\mathrm{P}$ in the water. Enrichment of $\mathrm{P}$ in natural waters can contribute to eutrophication, negatively affecting the aquatic ecosystem by stimulating primary production, which can lead to anoxic conditions (Tyrrell, 1999; Mainstone and Parr, 2002; Peuhkuri, 2002).

The use of enzymes like phytase, xylanase or $\beta$-glucanase in pigs and poultry is a common way to reduce the negative impact of phytate and NSP. With the breakdown of phytate and NSP through enzyme supplementation, the digestibility of the feed improves (Bedford and Schulze, 1998). Recently, the use of exogenous carbohydrases in aqua feeds is getting more attention. Multiple responses have been addressed to exogenous carbohydrases and phytase supplementation like improved feed intake, improved growth rate and nutrient digestibility (Lin et al., 2007; Pimentel-Rodrigues and Oliva-Teles, 2007; Goda et al., 2012; Castillo and Gatlin, 2015). However, besides the improved nutrient digestibility and growth, enzymes may improve energy efficiency in general (i.e. by lowering energetic cost for digestion or an alteration in body composition). The energy spend on digestion is part of the energy requirements for maintenance (MEm) of an animal. Besides 
environmental factors, nutritional factors can influence the MEm of fish (Schrama et al., 2012). For example, the dietary electrolyte balance (dEB) of the diet showed to affect the MEm in Nile tilapia, using nutritional identical diets (Saravanan et al., 2012). In pigs and poultry, dietary phytase supplementation has been shown to affect the energy utilisation (Kies et al., 2001). However, in fish information is lacking on the impact of enzyme application on the growth composition and energy partitioning and balance, in particular the effect on the MEm (i.e. energetic cost of digestion). In addition, synergy between enzymes have been observed on $\mathrm{P}$ and calcium (Ca) retention (Juanpere et al., 2005) and abdominal fat content (Tahir et al., 2005) in broilers. Similarly, synergy between different types of enzymes on fish can be expected.

Considering the above, the main objective of the presented study is to assess the impact of phytase and xylanase supplementation to the diet on the growth, body composition, digestibility and the energy, nitrogen and $\mathrm{P}$ balances. This was tested in a two by two factorial arrangement in Nile tilapia, to quantify the individual effects and the combination of the two enzymes.

\section{Materials and methods}

The experiment was approved by the Ethical Committee judging Animal Experiments of Wageningen University, The Netherlands, and carried out according to the Dutch law on animal experiments.

\section{Diets}

Four experimental diets were formulated in a $2 \times 2$ factorial arrangement. The first factor was phytase and the second was xylanase. This resulted in a control diet (CON-CON) without enzymes, a phytase diet (PHY-CON, Axtra ${ }^{\circledR}$ PHY, Buttiauxella $s p$. phytase at $1000 \mathrm{FTU} / \mathrm{kg}$, DuPont Animal Nutrition), a xylanase diet (Danisco ${ }^{\circledR}$ Xylanase at $6000 \mathrm{U} / \mathrm{kg}$, DuPont Animal Nutrition) and a diet with both phytase and xylanase (PHY-XYL). The experimental diets were identical in ingredient composition (Table 1). Yttrium oxide $\left(\mathrm{Y}_{2} \mathrm{O}_{3}\right)$ was included as an inert marker for digestibility studies. Diets were produced by SPAROS Lda. (Portugal), using extrusion. After extrusion, the respective enzyme in liquid form was coated onto the pellets prior to oil coating under vacuum. The experimental diets were formulated to meet the requirements of the essential nutrients according to the NRC (2011). Table 2 shows the analysed proximate composition and the measured enzyme activity of the experimental diets. The proximate composition was identical for all diets. The enzyme supplementation was successful with the intended recovery. Diets with (PHY-CON and PHY-XYL) and without (CON-CON and CON$\mathrm{XYL}$ ) phytase supplementation had an average measured phytase activity of 1301 and $116 \mathrm{FTU} / \mathrm{kg}$. Diets with (CON-XYL and PHY-XYL) and without (CON-CON and PHY-CON) xylanse had an average measured xylanase activity of 4235 and $<100$ $\mathrm{U} / \mathrm{kg}$ (Table 2). The diet formulation aimed to have a high phytate and NSP content and to magnify the response of the applied enzymes. Therefore, the protein in the diets was solely of plant origin. Sunflower meal, rapeseed meal and wheat DDGS 
(Dried Distillers Grains with Solubles) and a substantial amount of rice bran and wheat bran were included in the diets.

Table 1 Ingredient composition of the experimental diets.

\begin{tabular}{ll}
\hline Diet & \\
\hline Ingredient (\%) & 20.0 \\
Soybean meal & 10.0 \\
Wheat DDGS $^{+}$ & 10.0 \\
Wheat gluten & 10.0 \\
Rapeseed meal & 10.0 \\
Sunflower meal & 7.43 \\
Wheat & 7.50 \\
Maize & 8.50 \\
Wheat bran & 8.50 \\
Full-fat rice bran & 2.00 \\
Fish oil & 1.50 \\
Soy oil & 0.95 \\
Monocalcium phosphate & 1.50 \\
Lime (CaCO & ) \\
Lysine & 0.50 \\
Methionine & 0.40 \\
L-Threonine & 0.20 \\
Mineral and vitamin premix & \\
Yttrium oxide & 1.00 \\
\hline
\end{tabular}

${ }^{+}$Dried Distillers Grains with Solubles.

₹ Premix for freshwater fish, PREMIX Lda, Portugal. Vitamins (IU or $\mathrm{mg} / \mathrm{kg}$ diet): DL-alpha tocopherol acetate, $200 \mathrm{mg}$; sodium menadione bisulphate, $25 \mathrm{mg}$; retinyl acetate, $20000 \mathrm{IU}$; DLcholecalciferol, $1500 \mathrm{IU}$; thiamin, $20 \mathrm{mg}$; riboflavin, $30 \mathrm{mg}$; pyridoxine, $15 \mathrm{mg}$; cyanocobalamin, 0.05 $\mathrm{mg}$; nicotinic acid, $1750 \mathrm{mg}$; folic acid, $30 \mathrm{mg}$; ascorbic acid, $200 \mathrm{mg}$; inositol, $1000 \mathrm{mg}$; biotin, 2.5 $\mathrm{mg}$; calcium panthotenate, $50 \mathrm{mg}$; choline chloride, $1000 \mathrm{mg}$, betaine, $500 \mathrm{mg}$. Minerals $(\mathrm{g} \mathrm{or} \mathrm{mg} / \mathrm{kg}$ diet): cobalt carbonate, $2 \mathrm{mg}$; copper sulphate, $7.5 \mathrm{mg}$; ferric sulphate, $50 \mathrm{mg}$; potassium iodide, $0.3 \mathrm{mg}$; manganese oxide, $5.6 \mathrm{mg}$; sodium selenite, $0.1 \mathrm{mg}$; zinc sulphate, $5 \mathrm{mg}$; magnesium hydroxide, $10 \mathrm{mg}$; sodium chloride, $400 \mathrm{mg}$; potassium chloride, $900 \mathrm{mg}$; calcium carbonate, 2.15 g; excipient wheat middling's.

\section{Fish, rearing and housing facilities}

The experiment was performed at the Aquaculture Research Facility (ARF) of the Wageningen University, The Netherlands. Nile Tilapia (Oreochromis niloticus) from an all-male GIFT silver strain produced by Til-Aqua International BV (Someren, The Netherlands) were obtained. Fish were stocked in 24 rectangular 120-L tanks. Fish were fed a commercial diet prior to the experiment (Skretting E-1P Stella, containing $47 \%$ crude protein and $14 \%$ crude fat). The 24 tanks were divided over 3 racks of 8 tanks each. Each tank was randomly stocked with 30 fish. All tanks were connected to the same recirculation system, resulting in a common water supply and ensuring the same water quality for the inflow of each tank. The system 
consisted of a sump, settling tank and trickling filter with a water refreshment of 300 L/day. Each individual tank was connected to a swirl separator (AquaOptima AS, column height $44 \mathrm{~cm}$; diameter $24.5 \mathrm{~cm}$ ). The swirl separators, with detachable glass bottles were used to collect faeces and count feed spills for each tank separately. The flow through each tank was set at $7 \mathrm{~L} / \mathrm{min}$ using a hand held liquid rotameter. All tanks were equipped with a cylinder shaped air stone. The air stone and water flow ensured sufficient dissolved oxygen.

Water quality parameters were checked regularly to ensure that the water quality remained within pre-set ranges. During the experiment, the temperature ranged from 26.8-28.3 ${ }^{\circ} \mathrm{C}$, $\mathrm{pH}$ 6.8-7.9, dissolved oxygen between 4.7-7.5 mg/L, total ammonia nitrogen $<0.8 \mathrm{mg} / \mathrm{L}$, nitrite $<0.15 \mathrm{mg} / \mathrm{L}$ and nitrate $100-250 \mathrm{mg} / \mathrm{L}$, all within the pre-set ranges. Conductivity was $8000 \mu \mathrm{S} / \mathrm{cm}$ at stocking as a precaution, which was gradually lowered to $3000 \mu \mathrm{S} / \mathrm{cm}$ after week one. The photoperiod was set to $12 \mathrm{~h}$ light: $12 \mathrm{~h}$ dark (lights on 7:00, lights off 19:00).

Table 2 Analysed chemical composition and enzyme activity of the experimental diets.

\begin{tabular}{|c|c|c|c|c|}
\hline \multirow[t]{2}{*}{ Diet } & \multicolumn{2}{|c|}{ CON } & \multicolumn{2}{|c|}{ PHY } \\
\hline & CON & $X Y L$ & CON & $X Y L$ \\
\hline \multicolumn{5}{|c|}{ Analysed nutrient content ( $\mathrm{g} / \mathrm{kg}$ DM) } \\
\hline Dry matter (DM, g/kg) & 915 & 902 & 905 & 916 \\
\hline Crude protein & 370 & 373 & 368 & 371 \\
\hline Crude fat & 88 & 89 & 90 & 93 \\
\hline Carbohydrates $^{\dagger}$ & 460 & 456 & 460 & 455 \\
\hline Energy $(\mathrm{kJ} / \mathrm{g})$ & 20.4 & 20.4 & 20.2 & 20.4 \\
\hline Ash & 82 & 82 & 82 & 82 \\
\hline Phosphorus & 9.5 & 9.5 & 9.4 & 9.4 \\
\hline Calcium & 14.8 & 14.7 & 14.5 & 14.5 \\
\hline Yttrium & 0.186 & 0.180 & 0.161 & 0.159 \\
\hline \multicolumn{5}{|l|}{ Enzyme activity } \\
\hline Phytase (FTU/kg) & 130 & 101 & 1125 & 1477 \\
\hline Xylanase (U/kg) & - & 4228 & $<100$ & 4241 \\
\hline
\end{tabular}

Notes. PHY, phytase; XYL, xylanase; CON-CON, control diet; CON-XYL, diet supplemented with xylanase; PHY-CON, diet supplemented with phytase; XYL-PHY, diet supplemented with xylanase and phytase.

${ }^{+}$Carbohydrates calculated as $1000-$ (crude protein + crude fat + ash).

\section{Experimental procedure}

The experiment was conducted for 38 days. The four experimental diets were assigned randomly per rack of eight tanks. This resulted in two replicates per rack and six replicates per treatment. From a common batch fish were caught and randomly assigned to one of the 24 tanks. In total 30 fish were stocked per tank with an average initial weight of $42 \mathrm{~g}$. Fish per tank were group weighted while 
mildly sedated using $(0.25 \mathrm{ml} / \mathrm{L})$ 2-phenoxyethanol. At the end of the experiment all fish per tank were batch weighed and counted again while mildly sedated to determine final weight and calculate growth parameters. At the start of the experiment 20 fish from the same batch as the fish stocked were euthanized using an overdose of 2-phenoxyethanol (2 $\mathrm{ml} / \mathrm{L})$ for initial body composition determination. At the end of the experimental period, 10 fish per tank were randomly selected and euthanized for final body composition. Fish samples were stored at $-20{ }^{\circ} \mathrm{C}$ until further analysis.

The aim of the experiment was to test the effect of phytase, xylanase and their combination on growth, body composition, digestibility and the energy, nitrogen and $P$ balances. Therefore, the fish were restrictedly fed to keep the amount of feed on dry matter (DM) basis per tank per day equal. Fish were fed $16 \mathrm{~g} / \mathrm{kg}^{0.8} \mathrm{BW}$ day $^{-1}$, except the first six days when the daily ration was stepwise increased from $20 \%$ to $100 \%$ of the intended feed ration. The daily amount of feed was increased throughout the experiment by predicting fish growth and weight, using the average start weight of the fish (all treatments) and an expected feed conversion ratio (FCR) of 1.1. The daily feed ration was divided into two equal portions fed at 8:30 and 15:00 h. Fish were hand fed, feeding was completed within 1 hour per tank. Feed spills recovered from the settling units were recorded per tank after each feeding moment. The diets were kept under refrigerated $\left(4{ }^{\circ} \mathrm{C}\right)$ conditions throughout the experiment. Weekly a feed sample of $100 \mathrm{~g}$ was taken from each diet. The feed samples were pooled per diet and stored $\left(4{ }^{\circ} \mathrm{C}\right)$ until further analysis.

Faeces were collected for digestibility studies from week 4 onwards, using the swirl separators for 5 days per week (not the weekends). The glass bottles were submerged in ice to prevent bacterial degradation of the faeces. Faeces were collected between the afternoon and morning feeding (16:30 $\mathrm{h}-8: 00 \mathrm{~h})$. Faeces were pooled per week and stored in aluminium trays at $-20{ }^{\circ} \mathrm{C}$ until further analysis.

\section{Analytical procedures and calculations}

\section{Performance}

The absolute growth ( $\mathrm{g}$ ) was calculated as the difference between the average individual initial $\left(W_{i}\right)$ and final $\left(W_{f}\right)$ body weight per fish. The specific growth rate (SGR) was calculated as SGR $=\left(\operatorname{Ln} W_{f}-\operatorname{Ln} W_{i} \times 100\right) / t$, where $t$ is the duration of the experiment in days (d). The feed intake was recorded daily by weighing the feed given and refusals. In addition, the feed pellets recovered in the settling unit after feeding were counted. The FCR was calculated using the feed intake and weight gain per tank. The survival of fish per tank was calculated as $\left(\mathrm{N}_{\mathrm{f}} / \mathrm{N}_{\mathrm{i}}\right) \mathrm{X}$ 100 , where $N_{f}$ is the final number of fish and $N_{i}$ the initial number. 


\section{Digestibility}

The apparent digestibility coefficient (ADC) of DM, crude protein (CP), crude fat, total carbohydrate, gross energy, ash, $\mathrm{P}$ and $\mathrm{Ca}$ was calculated, with the use of yttrium as inert marker. The ADC was calculated according to the following formula (Cheng and Hardy, 2002): ADC (\%) $=100 \times\left[1-\left(Y_{i} \times\right.\right.$ amount nutrient in faeces) / $\left(Y_{f} \times\right.$ amount nutrient in feed $\left.)\right]$, where $Y_{i}$ is the concentration of yttrium in the feed and $Y_{f}$ the concentration of yttrium in the faeces. The total amount of carbohydrates in feed and faeces was calculated as: DM - (crude protein + crude fat + ash).

\section{Analyses}

Frozen fish samples $\left(-20^{\circ} \mathrm{C}\right)$ were ground twice using a meat mincer (Gastromaschinen, GmbH model TW-R 70; Feuma) with a $4.5 \mathrm{~mm}$ die and homogenised. Samples for the determination of DM and CP were taken from fresh samples, samples for crude fat and energy were freeze dried prior to the analyses. Collected faeces were oven dried at $70{ }^{\circ} \mathrm{C}$. Feed, faeces and fish samples were analysed according to the same methods. DM content was determined by drying samples for at least $4 \mathrm{~h}$ at $103^{\circ} \mathrm{C}$ until constant weight (ISO 6496, 1983). Ash content by incineration using a muffle furnace for $4 \mathrm{~h}$ at $550^{\circ} \mathrm{C}$ (ISO 5984, 1978). $\mathrm{CP}(\mathrm{N} \times 6.25)$ was analysed by the Kjeldahl method (ISO 5983, 1979). Crude fat was measured by petroleum-ether extraction (Soxhlet method, ISO 5986). Energy content was measured bomb calorimetric by direct combustion (IKA ${ }^{\circledR}$ werke, C7000; IKA analysentechnik, Weitershem, Germany). Yttrium, P and Ca in feed and faeces were analysed using inductively coupled plasma-mass spectrometry (ICP-OES) according to the standard NEN 15510 (2007).

The phytase and xylanase activity of feed samples were analysed by Danisco Animal Nutrition Laboratories (Brabrand, Denmark). Phytase was analysed using the methods described by Yu et al. (2012). One FTU phytase was defined as the amount of enzyme required to release $1 \mu \mathrm{mol}$ of inorganic $\mathrm{P}$ per minute from sodium phytate at $\mathrm{pH} 5.5$ at $37^{\circ} \mathrm{C}$. Xylanase was analysed using the methods described by Romero et al. (2013). One xylanase unit (U) was defined as the amount of enzyme that releases $0.48 \mu \mathrm{mol}$ of reducing sugar as xylose from wheat arabinoxylan per minute at $\mathrm{pH} 4.2$ and $50^{\circ} \mathrm{C}$.

\section{Energy, nitrogen and $P$ balance}

The energy, $\mathrm{P}$ and nitrogen $(\mathrm{N})$ balance parameters were calculated per tank and expressed on per fish basis as $\mathrm{kJ} / \mathrm{d}, \mathrm{mg} / \mathrm{d}$ and $\mathrm{mg} / \mathrm{d}$ respectively. The parameters were calculated as described by Saravanan et al. (2012). Generally balance parameters are expressed per metabolic body weight, however in the present study, fish were fed equal amount of DM of diets identical in nutrient composition and thus similar amount of energy, $\mathrm{P}$ and nitrogen. The final weights of the fish were affected by the enzyme supplementation (see result), using metabolic body 
weight would already create differences in balance parameters due to the differences in final body weight.

For the $\mathrm{N}$ balance, $\mathrm{N}$ intake was calculated as the product of feed intake and the dietary $\mathrm{N}$ content; digestible $\mathrm{N}$ intake as $\mathrm{N}$ intake times the digestibility coefficient of $\mathrm{N}$; retained $\mathrm{N}$ as the difference between final and initial $\mathrm{N}$ body mass; branchial urinary $\mathrm{N}$ losses as the digestible $\mathrm{N}$ intake minus retained $\mathrm{N}$. The $\mathrm{N}$ efficiency was calculated as retained $N$ (RN) divided by digestible $N$ (DN). The $P$ balance was calculated according to the same principle as the $\mathrm{N}$ balance. For the energy balance, energy intake was calculated as the product of feed intake and dietary energy content; digestible energy (DE) intake as energy intake times the energy digestibility coefficient; brachial urinary energy (BUE) losses as branchial urinary $\mathrm{N}$ losses times the energy content of $\mathrm{NH}_{3}-\mathrm{N}(24.9 \mathrm{~kJ} \mathrm{~N} / \mathrm{g})$, assuming that all $\mathrm{N}$ was excreted as $\mathrm{NH}_{3}-\mathrm{N}$; metabolisable energy (ME) intake as DE minus BUE; (RE) as the difference between final and initial body energy content; and heat production (HE) as ME minus RE. The MEm was calculated from the ME and the energy retained as protein (REp) and fat (REf). The following formula was used to calculate the $\mathrm{MEm}=\mathrm{ME}-((\mathrm{REp} / \mathrm{0.5})+(\mathrm{REf} / \mathrm{0.9}))$. In this calculation an energetic utilisation efficiency of ME for protein gain of $50 \%$ and an energetic utilisation efficiency of ME for fat gain of $90 \%$ was assumed (Lupatsch et al., 2003).

\section{Statistical analysis}

Statistical analyses were performed using the Statistical Analysis Systems (SAS) statistical software package version 9.3 (SAS Institute, Cary, NC, USA). All data were analysed for the effect of phytase and xylanase supplementation and their interaction by two-way ANOVA using PROC GLM. When the interaction between the enzymes phytase and xylanase was significant $(P<0.05)$, individual treatment means were compared using Tukey HSD (honest significant difference). All data were expressed as mean per treatment of the six replicates.

\section{Results}

\section{Performance}

Mean fish performance over the 38-day experimental period is given in Table 3. The average initial weight of the fish was $42 \mathrm{~g}$ and statistically identical $(P>0.1)$ among treatments. The survival of the fish during the experiment was high with a mean survival of $98.8 \%$. Conform to the experiment set-up the absolute feed intake was uniform among treatments $(1.56 \mathrm{~g} \mathrm{DM} / \mathrm{d})$. The daily growth $(\mathrm{g} / \mathrm{d})$ and the specific growth rate (SGR \%/d) were significantly affected by phytase and xylanase supplementation $(P<0.05)$. There was synergism between phytase and xylanase on growth $(\mathrm{g} / \mathrm{d})$ and the SGR, indicated by a significant interaction effect $(P<0.05)$. The effect of xylanase on daily growth was small, but in combination with phytase supplementation, xylanase enhanced growth. Combined phytase and xylanase had the highest growth $(1.58 \mathrm{~g} / \mathrm{d})$. The average FCR for diets without 
xylanase (CON-CON and PHY-CON) was 1.10 versus 1.06 for the diets with xylanase (CON-XYL and PHY-XYL). For diets without phytase (CON-CON and XYLCON) the average FCR was 1.14 versus 1.02 for the diets with phytase (PHY-CON and $\mathrm{PHY}-\mathrm{XYL}$ ). The FCR was the lowest for the diet (PHY-XYL) supplemented with both phytase and xylanase (0.99), there was no significant interaction effect between phytase and xylanase on the FCR $(P=0.138)$.

Table 3 Effect of dietary enzyme supplementation on the performance of Nile tilapia over 38 days. Values are means and the standard error of the mean (SEM). Means within the same row not sharing a common letter are significantly different $(P<0.05)$.

\begin{tabular}{|c|c|c|c|c|c|c|c|c|}
\hline \multirow[t]{2}{*}{ Diet } & \multicolumn{2}{|c|}{ CON } & \multicolumn{2}{|r|}{ PHY } & \multirow[b]{2}{*}{ SEM } & \multicolumn{3}{|c|}{$P$-values } \\
\hline & CON & $\mathrm{XYL}$ & $\mathrm{CON}$ & XYL & & $\mathrm{PHY}$ & $X Y L$ & $\mathrm{PHY} * \mathrm{XYL}$ \\
\hline Survival (\%) & 98.3 & 96.7 & 100 & 100 & - & ns & ns & ns \\
\hline Initial body weight (g) & 41.7 & 42.3 & 41.8 & 41.4 & 0.3 & ns & ns & ns \\
\hline Final body weight (g) & 93.2 & 94.6 & 98.1 & 101.4 & 0.8 & $* * *$ & $* *$ & ns \\
\hline Feed intake abs. ( $g$ DM/d) & 1.55 & 1.56 & 1.55 & 1.56 & 0.003 & - & - & - \\
\hline Growth $(\mathrm{g} / \mathrm{d})$ & $1.36^{\mathrm{a}}$ & $1.38^{\mathrm{a}}$ & $1.48^{\mathrm{b}}$ & $1.58^{c}$ & 0.018 & $* * *$ & $* *$ & $*$ \\
\hline SGR (\%/d) & $2.12^{\mathrm{a}}$ & $2.12^{\mathrm{a}}$ & $2.25^{\mathrm{b}}$ & $2.36^{c}$ & 0.020 & $* * *$ & $* *$ & $* *$ \\
\hline FCR & 1.15 & 1.13 & 1.04 & 0.99 & 0.014 & $* * *$ & $*$ & ns \\
\hline
\end{tabular}

Notes. PHY, phytase; XYL, xylanase; CON-CON, control diet; $C O N-X Y L$, diet supplemented with xylanase; PHY-CON, diet supplemented with phytase; XYL-PHY, diet supplemented with xylanase and phytase; Feed intake abs., feed intake absolute; SGR, specific growth rate; FCR, feed conversion ratio.

\section{Digestibility}

Apparent digestibility coefficient (ADC) of DM, CP, fat, carbohydrates, minerals and energy was significantly enhanced by the supplementation of phytase (Table 4, $P<0.001)$. The supplementation of xylanase only significantly enhanced the DM and macro nutrient digestibility $(P<0.05)$, with a tendency for significance for ash $(P=0.064)$ and $\mathrm{P}(P=0.053)$ digestibility. The $\mathrm{PHY}$ supplementation had a large impact on the mineral ADCs, with an increase in ADC of ash, P and Ca by 120, 72 and $275 \%$, respectively. Although phytase and xylanase act directly on phytate and carbohydrates, also the ADC of crude fat and $\mathrm{CP}$ increased with the supplementation of these enzymes. Phytase supplementation (average CON-CON and CON-XYL versus average PHY-CON and PHY-XYL) increased the ADC from 90.1 to $91.2 \%$ for $C P, 92.9$ to $93.9 \%$ for crude fat and 49.7 to $56.7 \%$ for carbohydrates. Xylanase supplementation (CON-CON and PHY-CON versus CON-XYL and PHYCON) increased the ADC from 90.4 to $91.0 \%$ for CP, 93.2 to $93.6 \%$ for crude fat and 51.9 to $54.4 \%$ for carbohydrates. There was no significant interaction between phytase and xylanase supplementation on the digestibility of nutrients $(P>0.05)$, but a trend towards significance $(P=0.068)$ for interaction on $P$ digestibility was present. Xylanase supplementation alone did not affect $P$ digestibility but in combination with phytase, xylanase further increased $P$ digestibility. 
Table 4 Effect of dietary enzyme supplementation on the apparent digestibility (ADC) coefficient of Nile tilapia. Values are means and standard error of the mean (SEM).

\begin{tabular}{|c|c|c|c|c|c|c|c|c|}
\hline \multirow[t]{2}{*}{ Diet } & \multicolumn{2}{|c|}{ CON } & \multicolumn{2}{|c|}{ PHY } & \multirow[b]{2}{*}{ SEM } & \multicolumn{3}{|c|}{$P$-values } \\
\hline & CON & $X Y L$ & CON & $X Y L$ & & $\mathrm{PHY}$ & $X Y L$ & $\mathrm{PHY*XYL}$ \\
\hline \multicolumn{9}{|l|}{$A D C(\%)$} \\
\hline Dry matter & 65.0 & 67.4 & 71.2 & 72.8 & 0.580 & $* * *$ & $* *$ & ns \\
\hline Crude protein & 89.8 & 90.4 & 90.9 & 91.5 & 0.147 & $* * *$ & $* * *$ & ns \\
\hline Crude fat & 92.6 & 93.1 & 93.7 & 94.1 & 0.153 & $* * *$ & $*$ & ns \\
\hline Carbohydrates & 47.9 & 51.4 & 55.8 & 57.4 & 0.902 & $* * *$ & $*$ & ns \\
\hline Energy & 72.4 & 74.6 & 76.0 & 77.5 & 0.428 & $* * *$ & $* * *$ & ns \\
\hline Ash & 18.6 & 23.8 & 44.5 & 48.6 & 2.276 & $* * *$ & $\#$ & ns \\
\hline Phosphorus & 39.3 & 39.3 & 66.1 & 68.9 & 0.726 & $* * *$ & $\#$ & $\#$ \\
\hline Calcium & 7.2 & 18.3 & 44.5 & 51.0 & 6.490 & $* * *$ & ns & ns \\
\hline
\end{tabular}

Notes. PHY, phytase; XYL, xylanase; CON-CON, control diet; CON-XYL, diet supplemented with xylanase; PHY-CON, diet supplemented with phytase; XYL-PHY, diet supplemented with xylanase and phytase.

\section{Body composition}

The protein content was unaffected by enzyme supplementation ( $P>0.1$; Table 5$)$. The phytase supplementation lowered the body fat content significantly with 11 $\mathrm{g} / \mathrm{kg}$ fresh weight $(P<0.001)$. This corresponded with the significant lower DM $(P=0.017)$ and energy $(P<0.001)$ content for the fish fed the phytase diets. The $D M, C P$ and fat content of the body were unaffected by the xylanase supplementation $(P>0.05)$. The phytase supplementation had a large impact on the whole body mineral content of the fish. Both the ash, $\mathrm{P}$ and $\mathrm{Ca}$ content of the body was significantly higher with phytase $(P<0.001)$. The xylanase supplementation slightly reduced the Ca contend of the fish significantly $(P=0.046)$ and had a tendency for significance $(P=0.061)$ to reduce the $\mathrm{P}$ content. There was no significant interaction found on the body composition between phytase and xylanase supplementation $(P>0.05)$. However, a tendency for a significant interaction effect on the Ca content was present $(P=0.093)$.

\section{Balances}

The nitrogen, energy and $P$ balance expressed on fish basis as $\mathrm{mg} / \mathrm{d}$ and $\mathrm{kJ} / \mathrm{d}$ are displayed in Table 6. Parallel to the CP digestibility, the digestible $\mathrm{N}$ intake was significantly increased both by phytase $(P=0.019)$ and xylanase $(P<0.001)$ supplementation. There was a significant interaction effect $(P=0.005)$ on the $\mathrm{N}$ retention between the two enzymes, indicating its synergistic properties. Compared to the CON-CON diet the increase in $\mathrm{N}$ retention was $2.3,7.4$ and $17.9 \%$ higher for the CON-XYL, PHY-CON and PHY-XYL diets respectively. In particular, the increase of $\mathrm{N}$ efficiency resulted in an increase in $\mathrm{N}$ retention, besides reduced branchial and urinary $\mathrm{N}$ losses. Phytase and xylanase supplementation had a significant synergistic effect $(P=0.011)$ on $\mathrm{N}$ efficiency, inducing the synergetic effect between the enzymes on the $\mathrm{N}$ retention. The $\mathrm{N}$ efficiency was comparable 
Table 5 Effect of dietary enzyme supplementation on body composition (on fresh weight basis) of Nile tilapia. Values are means and standard error of the mean (SEM).

\begin{tabular}{|c|c|c|c|c|c|c|c|c|}
\hline \multirow[t]{2}{*}{ Diet } & \multicolumn{2}{|c|}{ CON } & \multicolumn{2}{|c|}{ PHY } & \multirow[b]{2}{*}{ SEM } & \multicolumn{3}{|c|}{$P$-values } \\
\hline & CON & $X Y L$ & CON & $X Y L$ & & $\mathrm{PHY}$ & $X Y L$ & $P H Y * X Y L$ \\
\hline \multicolumn{9}{|c|}{ Unit in $\mathrm{g} / \mathrm{kg}$ fresh } \\
\hline Dry matter & 285 & 287 & 278 & 284 & 1.9 & $*$ & $\#$ & ns \\
\hline Crude protein & 159 & 159 & 157 & 160 & 1.0 & ns & ns & ns \\
\hline Crude fat & 95 & 97 & 83 & 87 & 2.1 & $* * *$ & ns & ns \\
\hline Energy (kJ/g) & 7.3 & 7.4 & 6.7 & 7.0 & 0.08 & $* * *$ & $*$ & ns \\
\hline Ash & 30 & 30 & 36 & 35 & 0.60 & $* * *$ & ns & ns \\
\hline Phosphorus & 5.0 & 4.9 & 6.1 & 5.9 & 0.09 & $* * *$ & $\#$ & ns \\
\hline Calcium & 7.7 & 7.6 & 10.4 & 9.6 & 0.21 & $* * *$ & $*$ & \# \\
\hline
\end{tabular}

Notes. PHY, phytase; XYL, xylanase; CON-CON, control diet; CON-XYL, diet supplemented with xylanase; PHY-CON, diet supplemented with phytase; XYL-PHY, diet supplemented with xylanase and phytase.

for the CON-CON and CON-XYL diet (42.7 and 42.9\%), the PHY-CON diet had a higher efficiency $(45.7 \%)$, whereas xylanase and phytase (PHY-CON diet) combined had the best $\mathrm{N}$ efficiency $(49.1 \%)$. This pattern of increasing $\mathrm{N}$ efficiency for the PHY-CON and PHY-XYL diet was reflected in significant lower branchial $\mathrm{N}$ losses $(P<0.05)$.

Both DE and ME increased significantly with phytase and xylanase supplementation $(P<0.001)$, without a significant interaction $(P>0.1)$. REp is by definition identical to the $\mathrm{N}$ retention multiplied by $23.7 \mathrm{~kJ} / \mathrm{g}$. The REf increased significantly with $\mathrm{XYL}$ supplementation $(P=0.011)$, whereas phytase supplementation significantly decreased REf $(P=0.001)$. The phytase supplementation resulted in a leaner fish growth, which is reflected in the increase of the REp and REf ratio from 1.05 to 1.36. In contrast, xylanase supplementation increased both the REp and REf, but with minor impact on the protein deposition (Table 5). The total energy retention had a tendency to show a significant interaction effect $(P=0.074)$. Only xylanase supplementation significantly increased $(P=0.001)$ the RE energy. The effect on RE was the strongest for the fish fed the XYL-PHY diet. MEm was also expressed in $\mathrm{kJ} / \mathrm{kg}^{0.8} / \mathrm{d}$ since MEm is proportionally related to the metabolic body weight of fish. Phytase had a tendency to significantly affect MEm in $\mathrm{kJ} / \mathrm{d}(P=0.065)$, with numerically the highest MEm for the PHY-CON diet. Expressed in $\mathrm{kJ} / \mathrm{kg}^{0.8} / \mathrm{d}$ there are no significant main effects of phytase and xylanase supplementation, however combined there is an tendency for an significant interaction effect $(P=0.092)$. The CON-CON, CON-XYL and XYL-PHY diets had comparable energy requirements $\left(\mathrm{kJ} / \mathrm{kg}^{0.8} / \mathrm{d}\right)$ for maintenance, while numerically the energy requirements for maintenance where higher for the PHY-CON diet.

The effect of enzyme supplementation on $P$ digestibility (Table 4 ) was reflected in the digestible $P$ intake. There was a significant interaction effect $(P=0.032)$ between phytase and xylanase on the digestible $P$ intake with the highest digestible $P$ intake $(10.1 \mathrm{mg} / \mathrm{fish} / \mathrm{d})$ for the $\mathrm{PHY}-\mathrm{XYL}$ diet. Xylanase supplementation only 
increased the digestible $P$ in combination with phytase, indicated by a significant interaction effect $(P=0.032)$. A significant interaction effect of phytase and xylanase was not present on the $P$ retention $(P=0.952)$, only phytase supplementation significantly increased the $\mathrm{P}$ retention $(66 \% ; P<0.001)$. The $\mathrm{P}$ efficiency remained unaffected by the enzyme supplementation. The $P$ efficiency was high with an average of $92 \%$ among treatments.

Table 6 Effect of dietary enzyme supplementation on nitrogen, energy and phosphorous balances of Nile tilapia. Values are means and standard error of the mean (SEM). Means within the same row not sharing a common letter are significantly different $(P<0.05)$.

\begin{tabular}{|c|c|c|c|c|c|c|c|c|}
\hline \multirow[t]{2}{*}{ Diet } & \multicolumn{2}{|c|}{$\mathrm{CON}$} & \multicolumn{2}{|c|}{ PHY } & \multirow[b]{2}{*}{ SEM } & \multicolumn{3}{|c|}{$P$-values } \\
\hline & CON & $\mathrm{XYL}$ & $\mathrm{CON}$ & $\mathrm{XYL}$ & & $\mathrm{PHY}$ & $\mathrm{XYL}$ & $\mathrm{PHY} \times \mathrm{XYL}$ \\
\hline \multicolumn{9}{|l|}{ Nitrogen $(\mathrm{N})$ balance $(\mathrm{mg} / \mathrm{d})$} \\
\hline Gross N intake & 91.8 & 92.8 & 91.1 & 92.5 & 0.19 & - & - & - \\
\hline Digestible $\mathrm{N}$ intake & 82.5 & 83.9 & 82.8 & 84.6 & 0.21 & $*$ & $* * *$ & ns \\
\hline Branchial and urinary $\mathrm{N}$ loss & $47.3^{\mathrm{a}}$ & $47.9^{\mathrm{a}}$ & $45.0^{\mathrm{b}}$ & $43.0^{\mathrm{b}}$ & 0.51 & $* * *$ & ns & $*$ \\
\hline $\mathrm{N}$ retention & $35.2^{\mathrm{a}}$ & $36.0^{\mathrm{a}}$ & $37.8^{\mathrm{b}}$ & $41.5^{c}$ & 0.46 & $* * *$ & $* * *$ & $* *$ \\
\hline $\mathrm{N}$ efficiency (RN/DN, \%) & $42.7^{\mathrm{a}}$ & $42.9^{\mathrm{a}}$ & $45.7^{\mathrm{b}}$ & $49.1^{c}$ & 0.57 & $* * *$ & $* *$ & $*$ \\
\hline \multicolumn{9}{|l|}{ Energy $(E)$ balance $(k J / d)$} \\
\hline Gross E intake & 31.7 & 31.8 & 31.3 & 31.8 & 0.065 & - & - & - \\
\hline Digestible E intake & 22.9 & 23.7 & 23.8 & 24.7 & 0.14 & $* * *$ & $* * *$ & ns \\
\hline Branchial and urinary E loss & $1.17^{\mathrm{a}}$ & $1.19^{\mathrm{a}}$ & $1.12^{\mathrm{b}}$ & $1.07^{\mathrm{b}}$ & 0.013 & $* * *$ & ns & $*$ \\
\hline Metabolisable E & 21.8 & 22.5 & 22.7 & 23.6 & 0.14 & $* * *$ & $* * *$ & ns \\
\hline Heat E & 11.7 & 12.0 & 13.2 & 12.8 & 0.25 & $* * *$ & ns & ns \\
\hline Retained E & 10.1 & 10.5 & 9.5 & 10.8 & 0.22 & ns & $* * *$ & $\#$ \\
\hline Retained $\mathrm{E}$ as protein & $5.2^{\mathrm{a}}$ & $5.3^{\mathrm{a}}$ & $5.6^{\mathrm{b}}$ & $6.2^{c}$ & 0.069 & $* * *$ & $* * *$ & $* *$ \\
\hline Retained $\mathrm{E}$ as fat & 4.8 & 5.2 & 3.9 & 4.6 & 0.18 & $* *$ & $*$ & ns \\
\hline E maintenance & 5.9 & 6.1 & 7.1 & 6.1 & 0.30 & $\#$ & ns & $\#$ \\
\hline E maintenance $\left(\mathrm{kJ} / \mathrm{kg}^{0.8} / \mathrm{d}\right)$ & 54.9 & 55.6 & 64.1 & 54.7 & 2.90 & ns & ns & $\#$ \\
\hline \multicolumn{9}{|c|}{ Phosphorus $(P)$ balance $(\mathrm{mg} / \mathrm{d})$} \\
\hline Gross P intake & 14.7 & 14.7 & 14.6 & 14.6 & 0.030 & - & - & - \\
\hline Digestible P intake & $5.8^{\mathrm{a}}$ & $5.8^{\mathrm{a}}$ & $9.6^{\mathrm{b}}$ & $10.1^{\mathrm{c}}$ & 0.11 & $* * *$ & $*$ & $*$ \\
\hline Branchial and urinary $\mathrm{P}$ loss & 0.3 & 0.5 & 0.7 & 1.3 & 0.29 & $\#$ & ns & ns \\
\hline$P$ retention & 5.4 & 5.3 & 9.0 & 8.8 & 0.25 & $* * *$ & ns & ns \\
\hline $\mathrm{P}$ efficiency (RP/DP, \%) & 94.0 & 92.0 & 93.0 & 88.0 & 3.70 & ns & ns & ns \\
\hline
\end{tabular}

Notes. PHY, phytase; XYL, xylanase; CON-CON, control diet; CON-XYL, diet supplemented with xylanase; PHY-CON, diet supplemented with phytase; XYL-PHY, diet supplemented with xylanase and phytase; RN, retained nitrogen; DN, digestible nitrogen; RP, retained phosphorus; DP, digestible phosphorus. 


\section{Discussion}

The diets where formulated to meet the available $\mathrm{P}$ requirements for growth, estimated to be $4.7 \mathrm{~g} / \mathrm{kg}$ DM feed by meta-analysis across fish species (Prabhu et al., 2013). With the measured $P$ levels of the diet (Table 2) and the ADC of $P$ (Table 4), the available $P$ level was calculated as $3.7,3.7,6.2$ and $6.5 \mathrm{~g} / \mathrm{kg}$ DM of feed for the CON-CON, CON-XYL, PHY-CON, and XYL-PHY diets, respectively. Therefore, the diets without phytase supplementation ( $\mathrm{CON}-\mathrm{CON}$ and $\mathrm{CON}-\mathrm{XYL}$ ) had available $P$ levels below the requirement of $4.7 \mathrm{~g} / \mathrm{kg} D M$. The total amount of $P$ in the diet of $9.5 \mathrm{~g} / \mathrm{kg}$ DM feed (Table 2) is well above the NRC (2011) of $4.0 \mathrm{~g} / \mathrm{kg}$ total P for tilapia. Due to the large impact of phytase supplementation on growth (Table 3), $\mathrm{P}$ body content (Table 5 ) and $\mathrm{P}$ retention (Table 6 ), it can be questioned whether the $3.7 \mathrm{~g}$ available $\mathrm{P} / \mathrm{kg}$ DM of feed was sufficient to meet the $P$ requirements. In addition, the whole $P$ content of the fish fed the diets without phytase was approximately 30-38\% lower compared to whole body $\mathrm{P}$ content of tilapia reported by Schneider et al. (2004), where the available $P$ in the feed was minimal $6 \mathrm{~g} / \mathrm{kg}$ DM. However, branchial and urinary losses (no distinction made between branchial and urinary losses) occurred, while with insufficient dietary P levels, one would expect waterborne $P$ uptake, resulting in negative values for branchial and urinary losses. Even Schneider et al. (2004) measured small P uptake from the water, while the body $\mathrm{P}$ content was higher than in the present study. Moreover, when fish are fed a diet deficient in $\mathrm{P}$, resulting in a decrease in $\mathrm{P}$ body content, $\mathrm{P}$ stored in bones can be used for ATP synthesis in order to realise growth by remineralisation. This can lead to bone deformities when the bone structure is not able to support the growth (Sugiura and Ferraris, 2004; Lall and Lewis-McCrea, 2007). The ability to use P stored in the bones (buffering effect) to realise growth can result in a delayed growth response when feeding a $\mathrm{P}$ deficient diet. It was seen in rainbow trout that the growth can stay unaffected until $80 \%$ of the initial body reserves of $\mathrm{P}$ is depleted (Hardy et al., 1993; Prabhu et al., 2013). Therefore one could suggest that the possible effect of a $\mathrm{P}$ deficiency is minimal in the present experiment, as the experiment only lasted for 38 days and the body $\mathrm{P}$ content of the control diet was only $21 \%$ lower compared to the diets high in available $P$ (phytase supplemented). Based on the observed strong impact of phytase supplementation on performance and increased whole body $\mathrm{P}$ content, it can be argued whether the present stated (available) P requirement in literature for Nile tilapia is correct.

Results of the present study showed that growth performance of the fish fed the control diet was relative low when comparing the FCR (1.15) with other studies performed at the research facilities (Wageningen University), using fish of comparable weight and feeding level (Schneider et al., 2004; Amirkolaie et al., 2006; Leenhouwers et al., 2007a; Saravanan et al., 2012; Schrama et al., 2012). This lower FCR is most likely related to the high level of NSP and inositol bound phosphate in the diet, as those components are considered poorly digestible for monogastric animals and fish (Choct, 1997; Stone, 2003; Liebert and Portz, 2005; 
Tahoun and Hammouda, 2009; Sinha et al., 2011). The dietary high levels of NSP and phytate were incorporated to potentiate the possible effect and synergy between the enzymes xylanase and phytase.

Phytase supplementation in fish is studied to a larger extend compared to carbohydrases like xylanase. Studies with phytase show improved $\mathrm{P}$ digestibility which is generally reflected in improved growth (Kumar et al., 2012). The effect of phytase supplementation on $\mathrm{P}$ digestibility depends on the dose of phytase, development stage of the fish, phytate level and type in the diet, which makes comparisons with other studies difficult (Satoh et al., 2002; Kumar et al., 2012). For Nile tilapia a dose of 750-1000 FTU/kg is recommended (Portz and Liebert, 2004; Liebert and Portz, 2005; Kumar et al., 2012), concurring with 1000 FTU/kg used in our study, which raised $\mathrm{P}$ digestibility by $68 \%$ (from 39.3 to $67.5 \%$ ).

Studies assessing the effect of carbohydrases on growth and nutrient digestibility in fish are minimal compared to pigs and poultry (Castillo and Gatlin, 2015). Studies testing the effect of xylanase generally apply the enzyme in an enzyme cocktail/mix. Hereby testing xylanase in combination with other enzymes like $\beta$ glucanase, cellulase, pectinase and protease (Stone, 2003; Ogunkoya et al., 2006; Lin et al., 2007; Yildirim and Turan, 2010; Ghomi et al., 2012). Results of various studies, which assessed the impact of carbohydrase supplementation on fish performance and nutrient digestibility, are not always in line with each other. Most studies found improved nutrient digestibility and performance (Lin et al., 2007; Yildirim and Turan, 2010; Ghomi et al., 2012), while other studies found no or minimal effect on performance and nutrient digestibility (Ogunkoya et al., 2006; Yigit and Olmez, 2011). The design of the present study allowed us to determine the impact on performance, digestibility, body composition and the balances of energy, nitrogen and $P$ of the individual enzymes xylanase and phytase as well as the combination. Dalsgaard et al. (2012) tested the main effect of xylanase supplementation on rainbow trout (Oncorhynchus mykiss), using four diets: control diet (fishmeal), soybean meal, sunflower meal and rapeseed meal. In line with the present study, in Dalsgaard et al. (2012) the xylanase supplementation enhanced lipid and CP digestibility. However, the effect of xylanase was depending on the diet composition (soybean, sunflower or rapeseed based), most likely the presence of xylans. Whereby, the increase in nutrient digestibility by xylanase did not result in improved growth parameters $(P>0.05)$. In Jiang et al. (2014), xylanase, when applied in the right dose resulted in an significant $(P<0.05)$ higher feed intake, accompanied with an increased SGR in juvenile Jian carp (Cyprinus carpio var. Jian).

Besides the main effects, both enzymes combined resulted in a synergic effect on growth performance, increasing the growth $(\mathrm{g} / \mathrm{d})$ from 1.36 to 1.58 compared to the control diet. The effect of applying xylanase without phytase on growth was minor, although significant $(P=0.004)$, but in combination with phytase supplementation, xylanase enhanced growth. Tahir et al. (2005), showed synergistic effects between cellulase and hemicellulase on abdominal fat and 
viscosity using broilers, indicated by an interaction effect. Another study using broilers (Juanpere et al., 2005), observed interaction between phytase and glycosidase on $\mathrm{P}$ and $\mathrm{Ca}$ retention. Hitherto, to our knowledge the synergic effects between enzymes on performance have not been seen in fish. The synergic effect on performance is not explained by the digestibility of the macronutrients, with only a tendency for synergy on the P digestibility $(P=0.068)$. The $\mathrm{N}$ balance showed that the synergic effect on growth was predominately due to the significant synergistic effects on $\mathrm{N}$ efficiency $(P=0.011)$ and $\mathrm{N}$ retention $(P=0.005)$. This was also reflected in the energy balance, showing interaction between phytase and xylanase on the REp $(P=0.005)$. What caused this increased protein deposition needs further elucidation.

The increase in growth with both phytase and xylanase was related to an improved macro nutrient digestibility and for phytase to an improved $\mathrm{Ca}$ and $\mathrm{P}$ digestibility. The xylanase supplementation increased the protein, fat and total carbohydrate ADC by $0.6,0.4$, and $2.5 \%$, respectively. Phytase supplementation increased the protein, fat and total carbohydrate ADC by $0.9,1.0$ and $7.0 \%$, respectively. For the carbohydrate digestibility, it can be assumed that the increase in digestibility is due to the increase in NSP digestibility. The remaining carbohydrate components, starch and sugars in extruded diets are almost $100 \%$ digested in Nile tilapia (Amirkolaie et al., 2006; Haidar et al., 2016). Xylanase hydrolyses arabinoxylans, which are part of the NSP fraction (Dornez et al., 2009). Phytase liberates phosphate from the inositol in the phytate molecule by hydrolysis (Kumar et al., 2012). Without phosphate the inositol becomes available for digestion, improving the NSP digestibility. However, the level of phytate and thus inositol as part of the NSP fraction in the diet is minimal. The effect of xylanase and phytase supplementation on the fat and protein digestibility can be explained by, i.e. increasing the access to protein and fat for digestive protease and lipase (Adeola and Cowieson, 2011). In addition, the possible induced negative effects of NSP on gut morphology, physiology and mucus layer, affecting the endogenous secretion of water, proteins, electrolytes and lipids will be lowered with improved NSP digestibility (Choct, 1997; Sinha et al., 2011).

The energy balance showed that the available energy for the fish was used differently for the two studied enzymes (Table 6). Both enzymes increased the DE intake as well as the ME intake. The xylanase supplementation resulted in an increase in REf and REp, whereas phytase supplementation resulted in an alteration in the ratio between REp and REf. Here the REf decreased whereas the REp increased, resulting in leaner fish (Table 5 ). Since the gain of protein goes together with a higher water gain compared to fat gain (FAO, 2001), the impact of phytase supplementation on growth on weight basis was larger compared to the xylanase supplementation. What caused this altered protein to fat deposition ratio in fish fed diets supplemented with phytase requires further research. In fish, it was observed that P deficiency led to an accumulation of body fat (Hardy, 2010). If the available $\mathrm{P}$ level was deficient for the CON-CON and CON-XYL treatments it 
might explain the altered protein to fat deposition by fish fed diets supplemented with phytase.

Assessing the effect of enzyme supplementation on the MEm was one of the goals of the presented study. It was suggested that with enzyme supplementation the MEm could be lowered, by lowering the energetic cost for digestion or by an altering the body composition. In this study, the MEm was on average $57.3 \pm 3.9$ $\mathrm{kJ} / \mathrm{kg}^{0.8} / \mathrm{d}$. The MEm found were close to the estimate of $60 \mathrm{~kJ} / \mathrm{kg}^{0.8} / \mathrm{d}$ by Lupatsch et al. (2010), and slightly lower than the average of $64.5 \mathrm{~kJ} / \mathrm{kg}^{0.8} / \mathrm{d}$ found by Schrama et al. (2012) for Nile tilapia. The hypothesis of this study was that enzymes could improve the growth through reduced MEm (energetic cost of digestion). However, the enzyme supplementation did not significantly affect MEm $(P>0.1$; Table 6$)$. Numerically the energy requirements were higher for the PHYCON diet, contrary to our expectation. The higher requirements for maintenance for the PHY-CON diet could be related to the observed increase in ash and $\mathrm{P}$ digestibility, increasing mineral uptake and possible affecting osmoregulation. Likewise, in Saravanan et al. (2013), a high dietary electrolyte balance induced by altering the ratio between $\mathrm{Na}, \mathrm{K}$ and $\mathrm{Cl}$ resulted in significant higher $\mathrm{MEm}$, suggesting that the increased $\mathrm{MEm}$ is due to an extra demand for energy to maintain the overall acid-base homeostasis. Although not directly involved in osmoregulation, $\mathrm{P}$ influences the functioning of the kidney, an important osmoregulatory organ. However, the XYL-PHY treatment with slightly higher ash and $\mathrm{P}$ digestibility did not show increased MEm compared to the other treatments. Information about $P$ absorption in fish is lacking. Further studies are needed to elucidate the effect of $\mathrm{P}$ and phytase supplementation on osmoregulation in fish.

In summary, the supplementation of both xylanase and phytase showed to be an effective tool to improve the nutrient availability of the diet, enhancing the growth performance in Nile tilapia. The MEm was not reduced with the use of enzyme supplementation. The combination of xylanase and phytase resulted in positive synergetic effects on growth, explained by the interaction effect on protein retention and efficiency.

\section{Acknowledgements}

The experiment was funded by Danisco Animal Nutrition (SP 1404), a division of DuPont Industrial Biosciences. Thanks are given to Menno ter Veld and the staff of the aquaculture research facilities for their technical support in running the experiment and operating the experimental systems. Furthermore, Tino Leffering and Ronald Booms are acknowledged for their support during the chemical analysis 

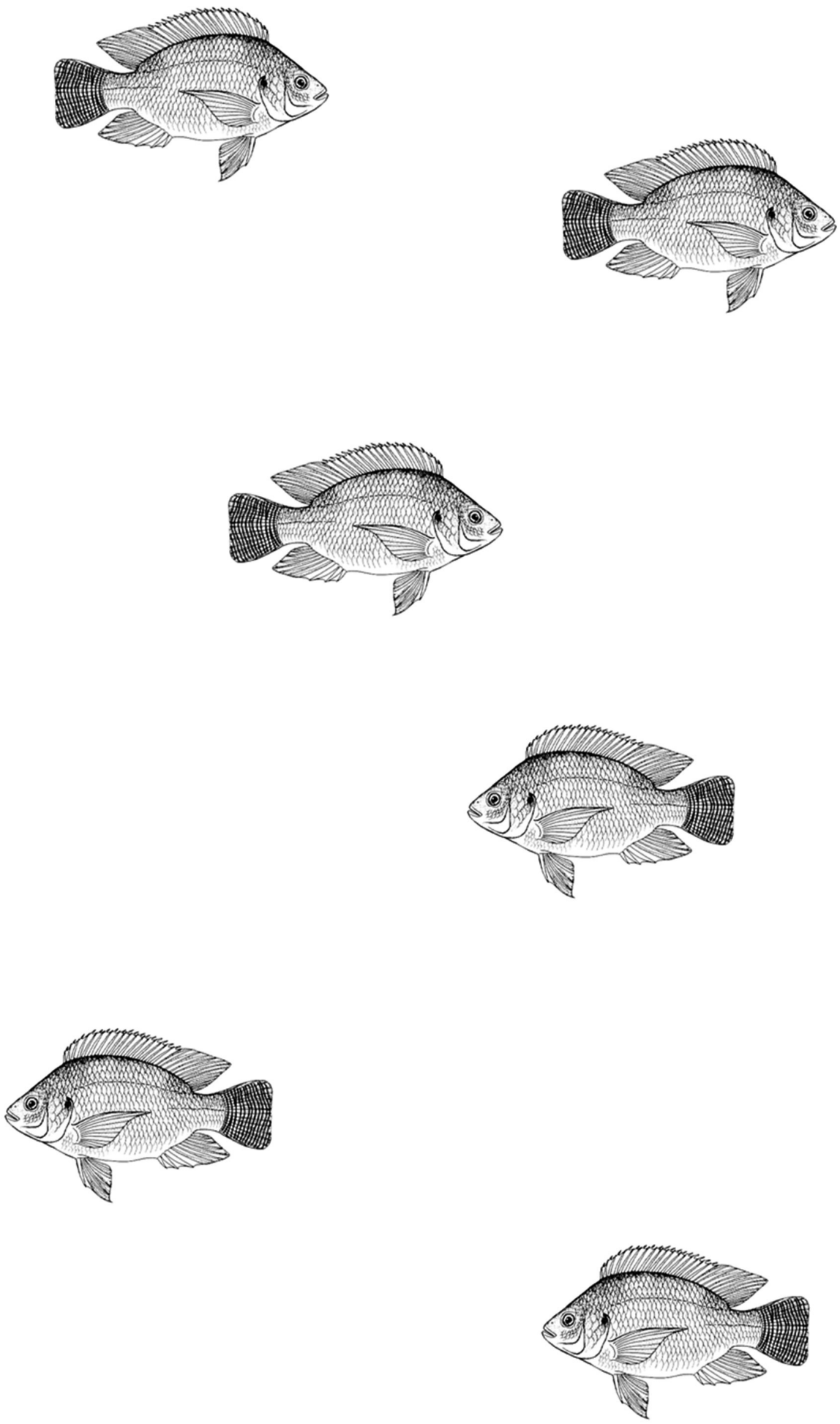


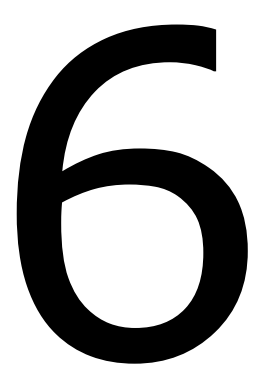

\title{
Effects and interactions between, phytase, xylanase and $\beta$-glucanase on growth performance and nutrient digestibility in Nile tilapia
}

\author{
Roel M. Maas, Marc C.J. Verdegem, Chia-Ning Lee, \\ Johan W. Schrama
}

Animal Feed Science and Technology (2020), 271: 114767 


\section{Abstract}

The main effects and synergy between three enzymes (phytase, xylanase and $\beta$ glucanase) on growth performance and nutrient digestibility was explored in Nile tilapia (Oreochromis niloticus). This was done according to a $2 \times 2 \times 2$ factorial arrangement, resulting in 8 dietary treatments. In total 24 tanks ( 3 replicates per treatment) were used with 30 fish each (mean initial body weight $38 \mathrm{~g}(\mathrm{SD} \pm 0.8)$ ). In a 42 day experiment growth and nutrient digestibility were measured. Fish were restrictively fed by hand twice daily. Phytase affected the nutrient digestibility, improving the ash, phosphorus and calcium digestibility. Neither individual enzymes supplementation, nor their combinations, did affect absolute growth $(\mathrm{g} / \mathrm{d})$. Averaged over diet, the feed conversion ratio (FCR) was low (0.92) despite $140 \mathrm{~g} / \mathrm{kg}$ non-starch polysaccharides (NSP) in the diets. A comparison with previous studies, which used comparable diets and enzyme treatments was made to elaborate on the minor effects of enzyme supplementation in the present study. The response of fish fed the diet with phytase and xylanase was comparable between studies. However, the control treatments without enzymes in the present study had a lower FCR and higher dry matter (DM) and NSP digestibility compared to previous studies. The current control diet was more comparable to the diets supplemented with enzymes in previous studies. It is likely that in the present study, and other studies that do not show improvement in growth with the use of enzymes, the control diets were not sufficiently limiting in nutrients for growth. 


\section{Introduction}

The expansion of the aquaculture sector has been accompanied by rapid growth of aqua feed production. The input of wild fish for the production of farmed fish is decreasing, leading to an increasing use of plant ingredients, such as soybean, wheat and corn in aqua feeds (Naylor et al., 2009; Pahlow et al., 2015). The substitution of fishmeal by plant protein has been the focus of many studies in the 2000s (e.g. Burel et al., 2000; Carter and Hauler, 2000; Chou et al., 2004; Hansen et al., 2007; Opstvedt et al., 2003; Øverland et al., 2009). Negative effects observed of using plant ingredients are mainly due to the presence of a range of anti-nutritional factors (ANF) like saponins, tannins, phytates, lectins, protease inhibitors and non-starch polysaccharides (NSP) (Francis et al., 2001; Sinha et al., 2011). Plant ingredients, in particular by-products (i.e., hull meals) can contain high levels $(>50 \%$ ) of poorly digestible NSP (Choct, 1997; Knudsen, 1997). NSP are poorly digested due to the absence of enzymes needed to hydrolyse the long polymeric chains. Therefore, energy stored in NSP remains unavailable for the fish its metabolic processes. Furthermore, NSP can negatively affect the gut and its functioning, which can lead to reduced nutrient digestibility (Francis et al., 2001; Sinha et al., 2011). In rats and poultry NSP is presumed to reduce the fat digestibility by entrapping bile acids which act as emulsifiers for fats (Ebihara and Schneeman, 1989; Smits and Annison, 1996). Therefore, NSP is undesired in feed formulation.

It is a common practice to use exogenous enzymes as feed additives in pig and poultry to improve the availability of nutrients in the feed (Adeola and Cowieson, 2011; Bedford and Cowieson, 2012). The use and effects of phytase supplementation to increase the bioavailability of phosphorus $(P)$ from phytate in aquaculture is well established, compared to the use of carbohydrases (Adeola and Cowieson, 2011; Kumar et al., 2012). Adeola and Cowieson (2011) defined carbohydrases as all enzymes that catalyse a reduction in the molecular weight of polymeric carbohydrate. Except for amylase that targets starch, the remaining carbohydrases are NSP-degrading enzymes, which break down NSP in oligosaccharides and monosaccharides (Woyengo and Nyachoti, 2011).

There are multiple kinds of NSP-degrading enzymes with different properties. However, xylanase and $\beta$-glucanase are the major commercial carboydrases, accounting for more than $80 \%$ of the global market (Adeola and Cowieson, 2011; Castillo and Gatlin, 2015). Xylanase degrades xylans into xylose, which is predominantly present in cereal grains such as oats, wheats and barley (Polizeli et al., 2005). Xylans are the second most abundant natural polysaccharide (Collins et al., 2005). $\beta$-glucanase hydrolyses $\beta$-glucan, which is present in the endosperm cell walls of cereals (Choct, 2006; Forrest and Wainwright, 1977). Both xylans and $\beta$-glucan can be classified as non-cellulosic polymers, also referred to as hemicelluloses (Sinha et al., 2011). 
Maas et al. (2018) found a synergy (interaction effect) between phytase and xylanase on the growth performance and the nitrogen $(\mathrm{N})$ balance in Nile tilapia. Growth and retained $\mathrm{N}$ at the control diet (no enzymes) and xylanase diet were similar, whereas the effect of phytase on growth and $\mathrm{N}$ retention was further enhanced when xylanase was supplemented in combination with phytase. Likewise, a synergy between $\beta$-glucanase and phytase and xylanase could be expected. A review by Castillo and Gatlin (2015) about exogenous carbohydrases in fish shows that generally an multi-enzyme complex or enzyme cocktail is used instead of single enzymes. Many of the mentioned studies in the review of Castillo and Gatlin (2015) used both xylanse and $\beta$-glucanase (i.e.; Lin et al., 2007; Ogunkoya et al., 2006; Stone et al., 2003; Yildirim and Turan, 2010). Therefore, the main effects of the individual enzymes and possible interactions between enzymes are less clear.

In this manuscript, the main effects and synergy between three enzymes; phytase, xylanase and $\beta$-glucanase on growth performance and nutrient digestibility in Nile tilapia (Oreochromis niloticus) were explored. The results of the presented study were not in line with previous experiments using the same diet and using a similar experimental setup. The same diet from the same batch was used in Maas et al. (2019) and a study (unpublished data) testing two fat levels, whereby the treatment with the low fat level was identical to the diet used in this study. Therefore, an additional comparison with the two previous performed studies was made to evaluate the discrepancy and repeatability of using the same diet and dietary (enzyme) treatment in different studies.

\section{Material and methods}

This experiment was evaluated by the Animal Welfare Body of Wageningen University and evaluated as not being an animal experiment according to Dutch legislation (Act on Animal Experiments).

\section{Diets}

Extruded diets ( $3 \mathrm{~mm}$ pellets) were produced by Research Diet Services (Wijk bij Duurstede, The Netherlands). Fat and enzymes were added together to the diets after extrusion by vacuum coating (Vacuüm core coater, Pegasus ${ }^{\circledR}-10 \mathrm{VC}, 1 / 4 \mathrm{H} / \mathrm{VV}$ nozzle nr. 6502), at the research facilities of the Animal Science Group, Wageningen University, The Netherlands. A mixture of fish oil, rapeseed oil and palm oil in a ratio of $2: 5: 5$ was applied to add an additional $60 \mathrm{~g} / \mathrm{kg}$ fat, resulting in approximately $80 \mathrm{~g} / \mathrm{kg}$ dietary fat. The coating allowed us to add the enzymes, using liquid forms with the fat to the diets. The enzymes were diluted 1:50 with demineralised water to increase the volume and to ensure a more homogenous dispersal of the enzymes during coating. For the treatments containing enzymes, $5-\mathrm{ml}$ per $\mathrm{kg}$ feed of solution was added to the oils to achieve the above mentioned enzyme activities. Control diets (no enzymes) were coated adding 5-ml 
demineralised water per $\mathrm{kg}$ feed. Diets were stored at refrigerated conditions (4 $\left.{ }^{\circ} \mathrm{C}\right)$ throughout the experiment.

Main effects and interactions between phytase (Axtra ${ }^{\circledR}$ PHY, Buttiauxella sp. phytase, DuPont Animal Nutrition), xylanase (Danisco ${ }^{\circledR}$ Xylanase, DuPont Animal Nutrition) and $\beta$-glucanase (Danisco Animal Nutrition) were tested according to a $2 \times 2 \times 2$ factorial arrangement, resulting in eight dietary (enzyme treatments) treatments. Enzymes were added aiming for an enzyme activity of $1000 \mathrm{FTU} / \mathrm{kg}$ DM diet for phytase, $4000 \mathrm{U} / \mathrm{kg}$ DM for xylanase and $500 \mathrm{BGU} / \mathrm{kg}$ DM for $\beta$ glucanase. The enzymes were supplemented to a diet (Table 1) with a relative high level of NSP (140 g/kg DM) due to the inclusion of $255 \mathrm{~g} / \mathrm{kg}$ wheat bran. The protein in the diet originated solely from plant ingredients. The analysed chemical composition of the diets was comparable (Table 2). Diets with and without phytase supplementation had an average measured phytase activity of 1149 and <50 $\mathrm{FTU} / \mathrm{kg} \mathrm{DM}$, respectively. Diets with xylanase supplementation had an average measured xylanase activity of $3330 \mathrm{U} / \mathrm{kg}$ DM, which was slightly lower than intended (4000 U/kg DM). Diets with $\beta$-glucanase supplementation had an average measured $\beta$-glucanase activity of $474 \mathrm{BGU} / \mathrm{kg}$ DM. The xylanase and $\beta$-glucanase activity was not measured for the diets without supplementation of those enzymes. The diet used in the current study is the same diet (same batch) as the wheat bran diet in Maas et al. (2019). The diet without the oils and enzymes was stored in the freezer at $-20{ }^{\circ} \mathrm{C}$ for 8 months before using in Maas et al. (2019) and approximately 12 months before using in the present study. After thawing this diet, new enzymes were mixed with the oil and were coated onto the kibbles by vacuum coating.

\section{Fish, rearing conditions and housing facilities}

The experiment was conducted in the Aquaculture Research Facility (ARF) of the Wageningen University, The Netherlands. Nile tilapia (Oreochromis niloticus) from a natural male silver strain (Silver $\mathrm{NMT}^{\mathrm{TM}}$ ) were obtained from a commercial fish breeder (Til-Aqua international, Someren, The Netherlands). Fish were housed in 160-L tanks and fed a commercial diet (ME-1.8 MP Presta, containing $550 \mathrm{~g} / \mathrm{kg}$ protein and $150 \mathrm{~g} / \mathrm{kg}$ fat, Skretting, Stavanger, Norway) two weeks prior to the start of the experiment. In total 24 rectangular tanks of 60-L placed in three rows of eight were used. Each tank was stocked with 30 fish, having an average start weight of $38.1 \mathrm{~g}$. The tanks were all connected to the same recirculation system resulting in a common water supply, ensuring the same water quality for the inflow of each tank. The water refreshment of the system was 300 L/day. The recirculation system consisted of a sump, settling tank and trickling filter. Every single tank was connected to a swirl separator (AquaOptima AS, column height 44 $\mathrm{cm}$; diameter $24.5 \mathrm{~cm}$ ) with a detachable glass bottle at the bottom, in order to count feed spills and collect faeces for each tank separately. The flow through each tank was set at $7 \mathrm{~L} / \mathrm{min}$ using a hand held liquid rotameter. All tanks were provided with a cylinder shaped air stone. The air stone and water flow ensured sufficient 
dissolved oxygen (DO) levels. The photoperiod was set to $12 \mathrm{~h}$ light: $12 \mathrm{~h}$ dark (lights on 7:00, lights off 19:00).

Table 1 Ingredient composition of the experimental diet.

\begin{tabular}{lr}
\hline Diet & \\
\hline Ingredients ( $\mathbf{g} / \mathbf{k g}$ ) & 140 \\
Maize starch & 76 \\
Wheat gluten & 187 \\
Wheat feed flour & 255 \\
Wheat bran & 85 \\
Casein & 76 \\
Soy protein concentrate & 76 \\
Pea protein & 10 \\
Fish oil & 25 \\
Rapeseed oil & 25 \\
Palm oil & 6.3 \\
Calcium carbonate & 19 \\
Monocalciumphospate & 3.2 \\
L-Lysine HCl & 4.4 \\
DL-Methionine & 1.9 \\
L-hreonine & 10 \\
Mineral and vitamin premix & + \\
Yttrium oxide & 0.2 \\
\hline
\end{tabular}

\footnotetext{
+ Premix composition. Vitamins (IU or $\mathrm{mg} / \mathrm{kg}$ Diet): vitamin B1, 10; vitamin B2, 10; vitamin B6, 10; vitamin B5, 10; vitamin B3, 20; biotin, 0.2; B-12, 0.015; folic acid, 2; vitamin C, 100; vitamin E, $100 \mathrm{IU}$; A-vitamin A palmitate, $3000 \mathrm{IU}$; D-Rovimix D3-500, $2400 \mathrm{IU} ; \mathrm{K}_{3} \mathrm{~K}$-menadione sodium bisulphite (51\%), 10; inositol, 400; choline, 1500; Antioxidant BHT (E300-321), 100; calcium propionate, 1000 . Minerals ( $\mathrm{mg} / \mathrm{kg}$ diet): ferric sulphate, 50 ; zinc sulphate, 30 ; cobalt sulphate, 0.1 ; copper sulphate, 10; Sodium selenite, 0.5 ; manganese sulphate, 20; magnesium sulphate, 500; chromic chloride, 1 ; calcium iodate, 2.
}

Water quality parameters were measured three times per week (MondayWednesday-Friday) in the morning before feeding to ensure that the water quality parameters remained within the pre-set ranges. The temperature (Testo 110) range was $27.4-27.6{ }^{\circ} \mathrm{C}$. The $\mathrm{pH}(\mathrm{WTW}-\mathrm{pH} 325)$ range was set between 7.0 and 7.8, sodium bicarbonate was added when the $\mathrm{pH}$ tended to drop below 7.0. The $\mathrm{pH}$ ranged between 5.9 (one short drop) and 7.6 during the experiment. DO levels of the common outflow ranged between 5.9 and $7.2 \mathrm{mg} / \mathrm{L}$ (WTW-Oxi 340i). Conductivity (WTW-Multi 3430) was $7000 \mu \mathrm{S} / \mathrm{cm}$ at stocking, which was gradually lowered to $3000 \mu \mathrm{S} / \mathrm{cm}$ after week one. The concentration of total ammonia nitrogen (TAN, Merck Aquamerck Colorimetric Ammonium test), nitrite $\left(\mathrm{NO}_{2}\right.$, Merck Aquamerck Colorimetric Nitrite test) and nitrate $\left(\mathrm{NO}_{3}\right.$, Merck MQuant Nitrate test strips) were measured. Total ammonia levels of the outflow remained below $0.3 \mathrm{mg} / \mathrm{L}$, nitrite $<0.2 \mathrm{mg} / \mathrm{L}$ and nitrate $<500 \mathrm{mg} / \mathrm{L}$ respectively. 


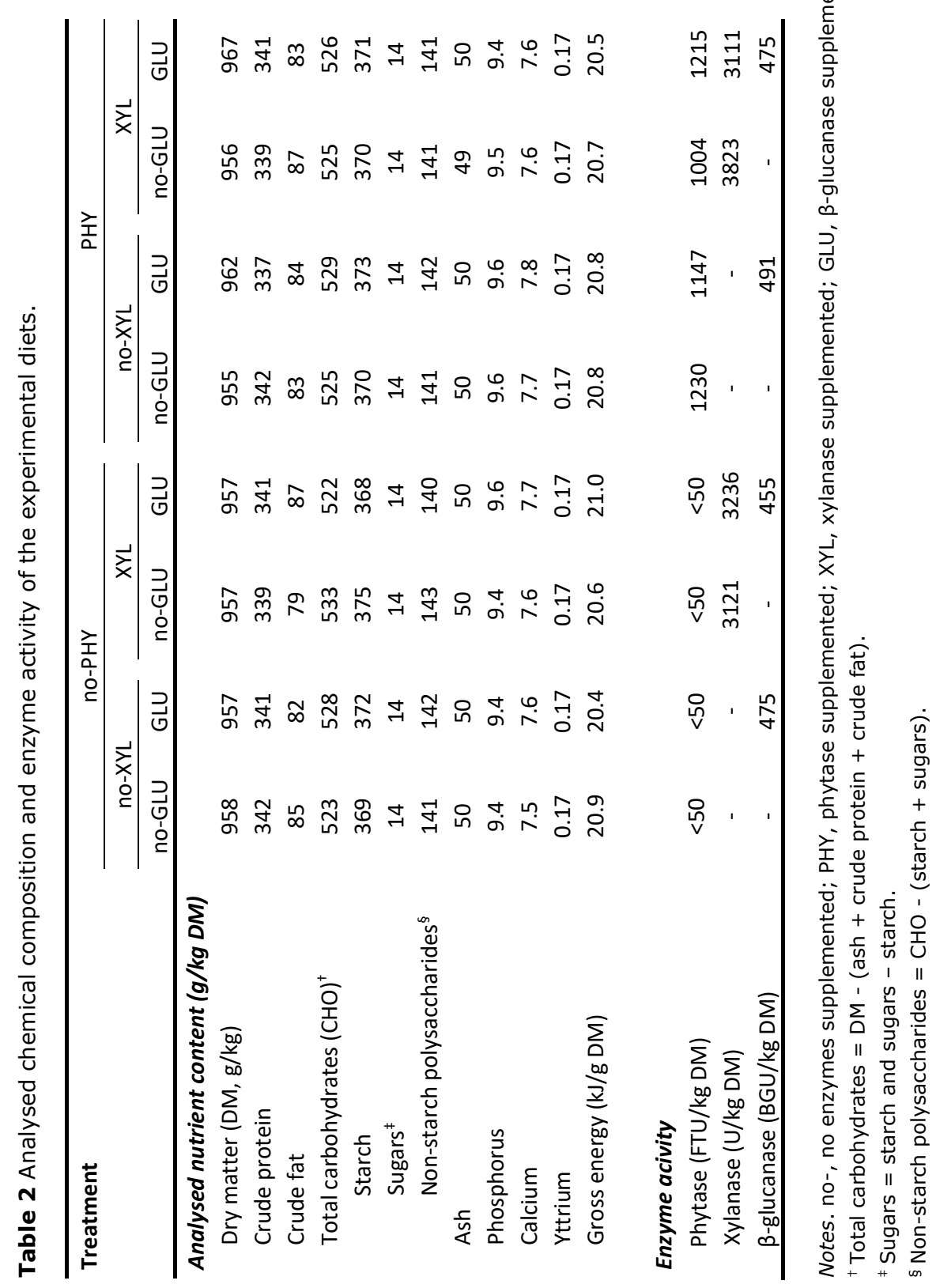




\section{Experimental procedure}

Feeding trial was conducted for 6 weeks (42 days). At the start 30 fish were randomly stocked per tank, fish per tank were bulk weighed to determine the initial weight of experimental populations. At the end of the experiment all fish per tank were bulk weighted to determine the final weight and to calculate growth parameters. The eight experimental diets were assigned randomly per rack of eight tanks. This resulted in 3 replicates per treatment.

The aim within the experiment was to test the effect of the applied enzymes on growth and digestibility of Nile tilapia. Therefore, the fish were restrictedly fed to keep the equal amount of feed on dry matter (DM) basis per tank per day. The feeding level was fixed and aimed at $16 \mathrm{~g} / \mathrm{kg}^{0.8} / \mathrm{d}$, being about $80 \%$ of the expected satiation level. The daily amount of feed was increased throughout the experiment by predicting fish growth and weight, using the average start weight of the fish and an expected feed conversion ratio of 1.0. At the first feeding moment, the fish were fed $20 \%$ of the intended feeding level, which was stepwise increased during 7 days to $100 \%$. The daily feed ration was divided into two equal portions fed at 9:00 and 15:30 $\mathrm{h}$. The fish were hand fed and feeding did not exceed $1 \mathrm{~h}$ for all tanks. Fifteen min after finalizing feeding, the settling bottles connected to the swirl separators were emptied and checked for uneaten pellets. The feed was sieved to remove small particles and dust and stored in plastic buckets in the fridge at $4{ }^{\circ} \mathrm{C}$. Each week a feed sample of $100 \mathrm{~g}$ was taken from each diet, this pooled sample was stored in the fridge at $4{ }^{\circ} \mathrm{C}$ until further analysis.

Faeces were collected for digestibility studies using a swirl separator, with a detachable glass bottle $(500-\mathrm{ml})$. The glass bottles were submerged in ice to prevent bacterial degradation of the faeces. Faeces were collected between the afternoon and morning feeding $(16: 30 \mathrm{~h}-8: 00 \mathrm{~h})$. Faeces were pooled per week and stored in aluminium trays at $-20{ }^{\circ} \mathrm{C}$ until further analysis.

\section{Analyses}

Collected faeces from the last 15 days were dried at $70{ }^{\circ} \mathrm{C}$ and pooled per tank. The faeces were ground using a mixer mill (Retsch Brinkmann, model MM2000) prior to the analysis. The pellets (diets) were not ground before analyses, except for starch. Collected faeces and feed were analysed for DM by drying at $103{ }^{\circ} \mathrm{C}$ for $4 \mathrm{~h}$ until constant weight. Ash content was determined by incineration in a muffle furnace for $4 \mathrm{~h}$ at $550{ }^{\circ} \mathrm{C}$ (ISO 5984, 1978). The total nitrogen content was measured using the Kjeldahl-method (ISO 5983), calculating crude protein as $\mathrm{N} \mathrm{x}$ 6.25. Crude fat was measured by petroleum-ether extraction (Soxhlet method, ISO 6492) and energy by bomb calorimeter (IKA ${ }^{\circledR}$ werke, C7000; IKA analysentechnik, Weitershem, Germany). Starch in feed and faeces was determined enzymatically using amyloglucasidase and starch + sugars in feed using the same method without ethanol extraction measuring glucose units, including those originating from free sugars. Yttrium, P and Ca were analysed using 
inductively coupled plasma-mass spectrometry (ICP-OES) according to the standard NEN 15510 (2007).

Danisco Animal Nutrition Laboratories (Brabrand, Denkmark) analysed the feed samples for phytase activity as described by Yu et al. (2012) and for xylanase activity as described by Romero et al. (2013). $\beta$-glucanase was analysed according to an in house colorimetric method by Danisco Animal Nutritional Laboratories.

\section{Performance}

Growth performance was calculated as described by Saravanan et al. (2012). The absolute growth (in $\mathrm{g}$ ) was calculated as the difference between the average individual initial $\left(\mathrm{W}_{\mathrm{i}}\right)$ and final $\left(\mathrm{W}_{\mathrm{f}}\right)$ body weight per fish. The geometric mean body weight $\left(W_{G}\right.$ in $g$ ) was calculated as $\sqrt{ }\left(W_{f} \times W_{i}\right)$ and the mean metabolic body weight $\left(\mathrm{MBW}_{\mathrm{g}}\right.$ in $\left.\mathrm{kg}^{0.8}\right)$ as $\left(\mathrm{W}_{\mathrm{G}} / 1000\right)^{0.8}$. The growth rate on metabolic weight (GR mbw in $\left.\mathrm{g} / \mathrm{kg}^{0.8} / \mathrm{d}\right)$ was calculated as $\left(W_{f}-W_{i}\right) /\left(M B W_{G} \times t\right)$, where $t$ is the duration of the experiment in days (d). The specific growth rate (SGR in \%/d) was calculated as $\ln \left(\mathrm{W}_{\mathrm{f}}\right)-\ln \left(\mathrm{W}_{\mathrm{i}}\right) \times 100 / \mathrm{t}$. The daily absolute feed intake $\left(\mathrm{FI}_{\mathrm{abs}}\right.$ in $\mathrm{g}$ $\mathrm{DM} / \mathrm{fish} / \mathrm{d})$ was calculated as $\mathrm{FI}_{\text {tot }} /(\mathrm{n} \times \mathrm{t})$, where $\mathrm{n}$ is the number of fish per tank and $\mathrm{FI}_{\text {tot }}$ is the total feed intake (in $\mathrm{g} \mathrm{DM}$ ), corrected for dead fish and feed spills. The feed conversion ratio (FCR) was calculated as $\mathrm{FI}_{\mathrm{mbw}} / \mathrm{GR}$ mbw, where the $\mathrm{FI}_{\mathrm{mbw}}$ is the feed intake per $\mathrm{kg}$ metabolic bodyweight (in $\mathrm{g} \mathrm{DM} / \mathrm{kg}^{0.8} / \mathrm{d}$ ) calculated as $\mathrm{FI}_{\mathrm{abs}}$ / $\mathrm{MBW}_{\mathrm{g}}$.

\section{Digestibility}

Yttrium was used as inert marker to calculate the apparent digestibility coefficient (ADC) of dry matter, crude protein, crude fat, total carbohydrate, starch, NSP, gross energy, ash, $\mathrm{P}$ and calcium ( $\mathrm{Ca}$ ) for each tank. The ADC was calculated according to the following formula (Cheng and Hardy, 2002): ADC $(\%)=100 \times[1$ - ( $Y_{i} X$ amount nutrient in faeces)/ ( $Y_{f} X$ amount nutrient in feed)], where $Y$ is the concentration of yttrium in the diet and faeces and $\mathrm{N}$ is the quantity of nutrient or energy content in the diet and faeces. The total amount of carbohydrates in feed and faeces was calculated on DM basis as: 1000 - (crude protein + crude fat + ash). The NSP fraction was calculated as: total carbohydrates - (starch + sugars).

\section{Statistical analyses}

A three-way ANOVA using a General Linear Model (GML) was used to test for the effect of phytase, xylanase and $\beta$-glucanase and their interactions on the growth performance and digestibility. Tukey HSD (honest significant difference) was used to compare individual treatment means of the different experiments. All data were

expressed as the mean per treatment of three replicates. All statistical analyses were carried out using the IBM Statistical Package for the Social Sciences (SPSS) program (v 23.0, New York, United States). 


\section{Results}

\section{Performance}

The average start weight of the fish was $38.1 \mathrm{~g}$ and identical $(P>0.05)$ among treatments (Table 3 ). The total survival was $98.2 \%$ and unaffected by treatments $(P>0.1)$. The absolute feed intake $(1.71 \mathrm{~g} \mathrm{DM} / \mathrm{d})$ was equal among treatments. None of the enzymes (phytase, xylanase and $\beta$-glucanase) nor their combinations affected the growth parameters $(P>0.05)$, except for a tendency $(P<0.1)$ for a significant interaction between phytase and xylanase on the GR mbw $\left(\mathrm{g} / \mathrm{kg}^{0.8} / \mathrm{d}\right)$ and SGR $(\% / d)$. Final body weights after the 42 day experimental period were highly comparable (114.1-117.4 g) among treatments. The FCR of the treatments ranged between 0.90 and 0.94 .

\section{Digestibility}

The ADC of DM, protein, fat, carbohydrates, starch, NSP and energy was unaffected by the enzyme treatments (Table $4, P>0.05$ ). From the above parameters there was a tendency for an interaction of phytase and xylanase on crude protein and starch and for phytase on crude protein and energy $(P<0.1)$. Phytase supplementation enhanced the digestibility of ash and the minerals $P$ and $\mathrm{Ca}(P<0.05)$. The starch digestibility was almost complete with an ADC of $99.9 \%$ to $100 \%$. Phytase enhanced the P ADC (average all diets with phytase) compared to the control diets (average all diets without phytase) from $72.6 \%$ to $77.9 \%$. The DM ADC of all dietary treatments ranged between $86.1 \%$ and $86.7 \%$.

\section{Discussion}

In the presented experiment the effect of applying enzyme(s) (phytase, xylanase and $\beta$-glucanase) singular or in combination on growth was absent $(P>0.1)$, which is in line with some literature on fish (Adeoye et al., 2016b; Dalsgaard et al., 2012; Farhangi and Carter, 2007; Yigit and Olmez, 2011). In contrast, other studies showed an improvement on growth performance with the use of NSP-degrading enzymes and phytase (Adeoye et al., 2016a; Ai et al., 2007; Ghomi et al., 2012; Jiang et al., 2014; Lin et al., 2007; Maas et al., 2018; Yildirim and Turan, 2010; Zhou et al., 2013). The diet used in the presented study is the same diet as the wheat bran (WB) diet described in Maas et al. (2019), from now on referred to as experiment 2 . The diet was also used in a study (unpublished data) without enzymes, from now on referred to as experiment 1 . The diet used in this study and the experiments 1 and 2 is from the same batch. The diet without the additional supplementation of the oils (fish oil, rapeseed oil and palm oil) and enzymes was stored in the freezer at $-20{ }^{\circ} \mathrm{C}$ in between the experiments. An overview of the three studies with the overlapping diet and enzyme treatments is given table 5 . 


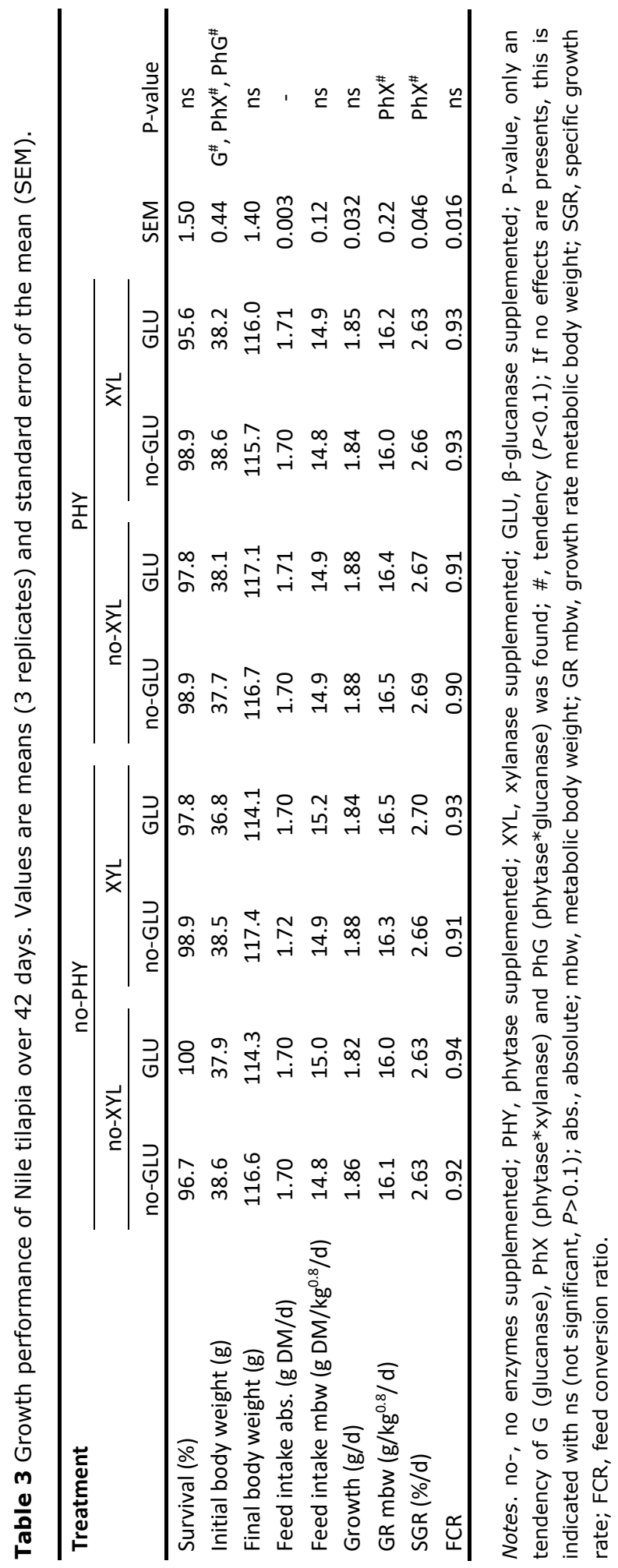




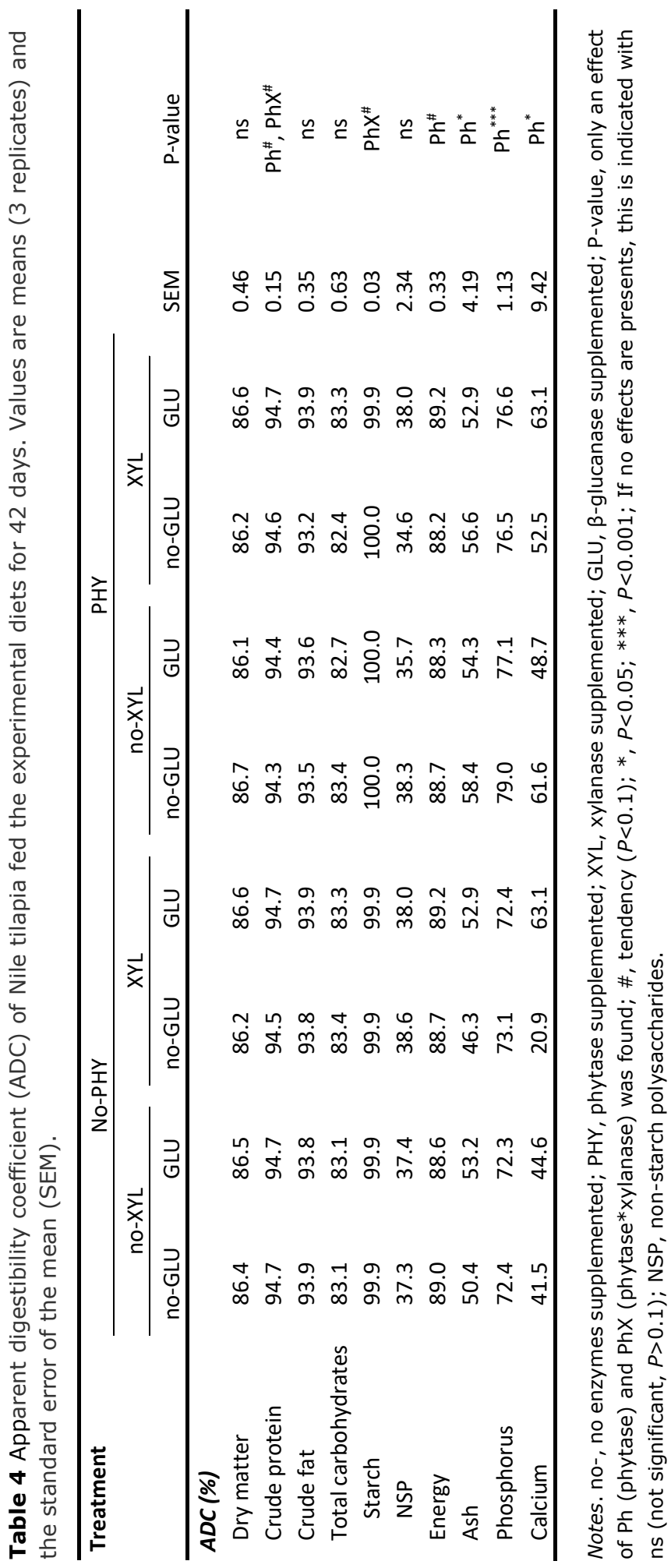


Table 5 Overview performed experiments using the same diet.

\begin{tabular}{lllccccc}
\hline & $\begin{array}{l}\text { Start } \\
\text { experiment }\end{array}$ & Treatment & $\begin{array}{c}\text { Duration } \\
\text { (d) }\end{array}$ & $\begin{array}{l}\text { Initial mean } \\
\text { weight (g) }\end{array}$ & $\begin{array}{l}\text { Final mean } \\
\text { weight (g) }\end{array}$ & $\begin{array}{l}\text { Feed intake } \\
\text { (g DM/kg.8/d) }\end{array}$ & FCR \\
\hline Experiment 1 & $8-3-2016$ & no enzymes & 43 & 48.1 & 104.1 & 11.2 & 1.05 \\
\hline $\begin{array}{l}\text { Experiment 2 } \\
\text { (Maas et al., 2019) }\end{array}$ & $8-11-2016$ & $\begin{array}{l}\text { phytase \& } \\
\text { xylanase }\end{array}$ & 43 & 41.1 & 101.8 & 11.9 & 0.92 \\
& & $\begin{array}{l}\text { no enzymes } \\
\text { present }\end{array}$ & 43 & 41.0 & 97.2 & 12.2 & 1.00 \\
\hline experiment $^{\ddagger}$ & $21-3-2017$ & $\begin{array}{l}\text { phytase \& } \\
\text { xylanase }\end{array}$ & 42 & 38.6 & 115.7 & 14.8 & 0.93 \\
& & no enzymes & 42 & 38.6 & 116.6 & 14.8 & 0.92 \\
\hline
\end{tabular}

${ }^{+}$natural male strain (Red NMT'M $)$.

${ }^{\ddagger}$ natural male strain (Silver NMT ${ }^{\mathrm{TM}}$ ).

From experiment 2 the WB diet was chosen for the use in this following up study as it showed the largest contrast in growth and digestibility between the with (phytase and xylanase) and without enzyme treatment (Maas et al., 2019). The growth in the present experiment was similar irrespective of the enzyme treatment (Table 3). The FCR was in the range of 0.90-0.94, which is comparable to the FCR of the WB diet with enzyme treatment (0.92) in experiment 2. However, the FCR of the control diet (without enzymes) in the present experiment was considerably lower with 0.92 versus 1.00 in experiment 2 . The lower FCR may partially be explained by the higher feeding level $\left(14.8 \mathrm{~g} \mathrm{DM} / \mathrm{kg}^{0.8} / \mathrm{d}\right)$. Haidar et al. (2016) showed that when not feeding close to satiation $\left(18.8-19.4 \mathrm{~g} \mathrm{DM} / \mathrm{kg}^{0.8} / \mathrm{d}\right.$ ) the FCR will improve with an increase in feeding level. The lower FCR of the control diet in the present study is in line with a higher digestibility of the diet. In experiment 1 , the DM ADC of the control diet was $80.4 \%$, and $82.6 \%$ in experiment 2 , whereas the DM ADC of the control diet was $86.4 \%$ in the present experiment. The higher digestibility is mainly due to a higher ash, NSP and thus carbohydrate digestibility. In experiment 2, the DM ADC increased from 82.6- to $84.3 \%$ with the use of phytase and xylanase, which is still below the digestibility of the diet without enzymes in the present study (Figure 1).

For all three experiments, the same procedures were followed. In experiment 1 , the initial body weight of the fish was slightly higher $(48.1 \mathrm{~g})$ compared to the fish in the present study and experiment 2, which were of comparable weight $(38.1 \mathrm{~g}$ and $41.1 \mathrm{~g}$ ). The fish used in experiment 1 were from a different strain (natural male strain, Red $\mathrm{NMT}^{\mathrm{TM}}$ ) than the present study and experiment 2 (natural male silver strain $\mathrm{NMT}^{\mathrm{TM}}$ ), all fish were obtained from the same fish breeder. The feeding level differed slightly between the experiments. The feeding level for the diet without enzymes was $11.2,12.2$ and $14.8 \mathrm{~g} \mathrm{DM} / \mathrm{kg}^{0.8} / \mathrm{d}$ for experiment 1 , experiment 2 and the present study respectively. Studies on the effect of feeding level on nutrient digestibility have shown a reduction in the nutrient digestibility with increasing feeding level (Henken et al., 1985; Schrama et al., 2012; Windell et al., 1978), where other studies found no effects (Azevedo et al., 1998; Storebakken and Austreng, 1987). However, to our knowledge studies indicating 
an improvement in digestibility with increasing feeding level are absent. In the present comparison of the same diet used in different studies, the digestibility increased both with an increase in feeding level and over time. What caused this observed increase in digestibility over time, despite the higher feeding level remains unclear.

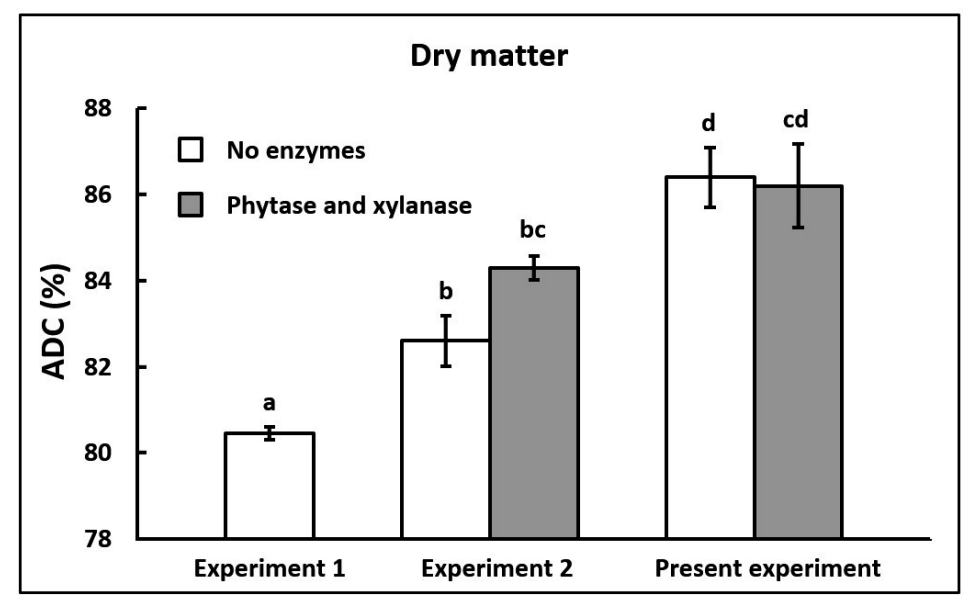

Figure 1 Dry matter apparent digestibility coefficient (ADC, \%) of the same diet for three different feeding trials. Values are means (3 replicates) with standard deviation represented by error bars. Means not sharing a common letter are significantly different $(P<0.05)$.

Haidar et al. (2016) showed that with an increase in feeding level the ADC of NSP rapidly decreased, suggesting a threshold in the amount of NSP that can be digested. However, in the present experiment the endogenous NSP ADC (no enzymes) was higher (37.3\%) than the same WB diet in experiment $1(11.1 \%)$ and experiment $2(17.1 \%)$ despite the higher feeding level. The NSP ADC in experiment 2 improved from $17.1 \%$ to $25.1 \%$ with the use of enzymes, which is below the endogenous NSP ADC in the present study (Figure 2).

Other than in experiment 2, the enzymes phytase and xylanase did not improve the NSP digestibility, nor did $\beta$-glucanase singular or in combination with the other enzymes. The NSP fraction that potentially could be hydrolysed by the supplemented enzymes may have been digested anyway, considering the high endogenous digestibility, thereby masking the potential enzyme effect on NSP digestibility. Previous studies using Nile tilapia of comparable weight and applying a similar feeding level showed that NSP can be partially digested (Amirkolaie et al., 2005; Haidar et al., 2016; Leenhouwers et al., 2007a). However, in those studies the maximum NSP digestibility was on average lower, only in Haidar et al. (2016) a higher NSP ADC (56\%) was found when using a comparable feeding level, suggesting that the present endogenous NSP is among the highest NSP digestibility's reported. 


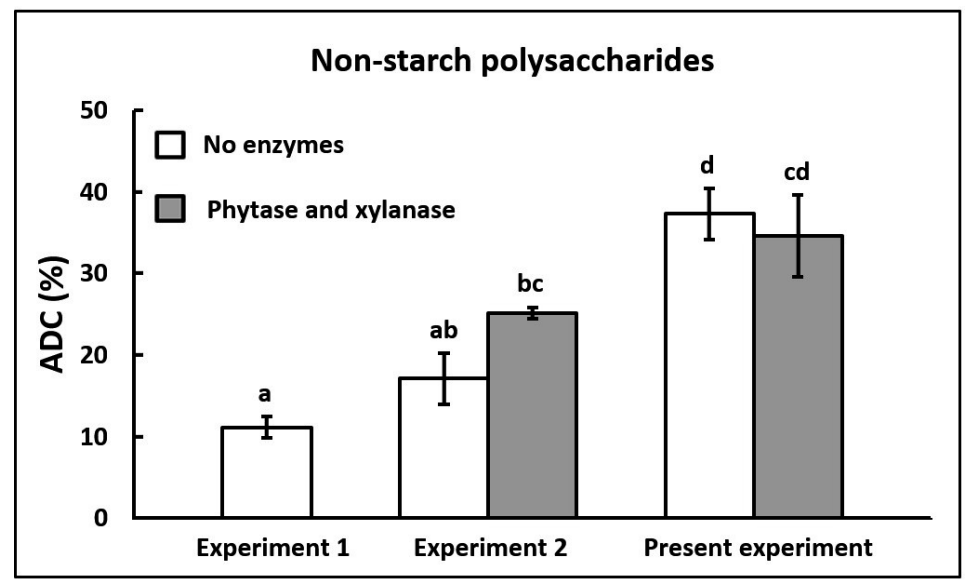

Figure 2 Non-starch polysaccharide (NSP) apparent digestibility coefficient (ADC, \%) of the same diet for three different feeding trials. Values are means (3 replicates) with standard deviation represented by error bars. Means not sharing a common letter are significantly different $(P<0.05)$.

In line with the digestibility, the performance of the fish fed the control treatment suggests that there is little improvement with the use of enzymes. The FCR was 0.92 for the control group in this study, which is similar to other studies performed at the Wageningen University (Saravanan et al., 2012; Saravanan et al., 2013; Schrama et al., 2012). However, the FCR in the present study is much lower compared to studies using a diet high in NSP (Haidar et al., 2016; Leenhouwers et al., 2007a; Maas et al., 2018). Comparable to our study, in Adeoye et al. (2016b) enzyme supplementation (phytase, xylanase and protease) did not improve the growth of tilapia compared to the control treatment (no enzymes). Similar, the FCR of the control treatment was low (0.94), minimizing the potential improvement with the use of enzymes. However, in Adeoye et al. (2016b) the low FCR was accompanied with a low dietary fibre fraction $(<4 \%)$.

From the enzymes phytase, xylanase and $\beta$-glucanase and their combinations only phytase influenced digestibility. Phytase improved the ADC of the ash fraction and the minerals $\mathrm{P}$ and $\mathrm{Ca}$. The P ADC improved with $6.4 \%$ on average compared to the control diets (no phytase). The increase in available $\mathrm{P}$ did not result in an improvement of growth, which is in line with Hu et al. (2016), where Portz and Liebert (2004) found improved growth. The available $P$ level of the control diets was $6.8 \mathrm{~g} / \mathrm{kg} \mathrm{DM}$ feed, calculated using the P level in the diet $(9.4 \mathrm{~g} / \mathrm{kg} \mathrm{DM})$ and the ADC of $P(72.4 \%)$. The available $P$ level is well above the stated requirement of $4.0 \mathrm{~g} / \mathrm{kg}$ total $\mathrm{P}$ for tilapia by the NRC (2011) and the estimated available P requirement for growth of $4.7 \mathrm{~g} / \mathrm{kg}$ DM feed by meta-analysis across fish species of Prabhu et al. (2013). Therefore, it is unlikely that an available P level of 6.8 $\mathrm{g} / \mathrm{kg}$ DM feed is limiting growth and that higher levels of available $P$ levels will improve growth. Besides the increase in P ADC the ash and Ca ADC improved with phytase. The main effect of phytate is through reduced bio-availability of minerals 
like $\mathrm{Ca}, \mathrm{Cu}, \mathrm{Mg}, \mathrm{Fe}, \mathrm{Sr}, \mathrm{Zn}$ etc. by binding to cations, forming insoluble complexes (Francis et al., 2001; Kumar et al., 2012; Nolan et al., 1987). When phytase hydrolyses phytate, the bioavailability and absorption of minerals like Ca will be enhanced (Sugiura et al., 2001), thereby increasing the ADC of the ash fraction. Improving the availability of minerals like $P$ is of importance in order to improve the sustainability of the aquaculture sector. The plant (protein) ingredients used as replacers for fish meal generally have a lower $\mathrm{P}$ content. Furthermore, the $\mathrm{P}$ content in plant ingredients is mostly present on the form of phytate, which is poorly utilised by fish (Kumar et al., 2012). In order to fulfil the $P$ requirements, inorganic phosphorus sources like dicalcium phosphate, sodium phosphate and potassium phosphate are often supplemented to the diet (Prabhu et al., 2013). However, using $\mathrm{P}$ mined from exhaustible phosphate rock deposits is undesired given the limitations of the natural reserves of phosphate (Obersteiner et al., 2013). Improving the availability of $P$ from plants in the diet with for instance phytase reduces the need for inorganic $P$ sources. In addition, improving the $P$ availability and retention is important to minimise the discharge of $P$ and the stimulating effect $P$ can have on eutrophication when discharged in natural waters (Mainstone and Parr, 2002; Peuhkuri, 2002). In the current experiment the supplementation of phytase to the diet reduced the direct $\mathrm{P}$ waste through the faeces with $17.5 \%$.

In between the experiments, the kibbles of the uncoated diets (without the palm, rapeseed- and fish oil) were continuously stored in the freezer at $-20{ }^{\circ} \mathrm{C}$. The conditions during storage of ingredients and diets is known to influence its quality. The vitamin content may be reduced or a change in the fat quality can occur due to oxidation. High storage temperatures will reduce the amino acids digestibility due to Maillard reaction. Those processes will reduce the nutritive value of the diet or ingredient (Tangendjaja, 2015). Negative effects on the nutritive value of the diets due to storage are not expected as the diets were stored at $-20{ }^{\circ} \mathrm{C}$. In Hardy et al. (1983) lipid oxidation of diets stored for four months at $-20{ }^{\circ} \mathrm{C}$ was absent. If the storage over time affected the nutritive quality of the diet at all, negative effects are expected. However, in the current studies the digestibility of the diet increased over time despite the higher feeding level. To our knowledge there are no studies indicating an improvement in digestibility of diets when stored for longer periods $(12$ months $)$ in the freezer $\left(-20^{\circ} \mathrm{C}\right)$.

To summarize, no effects on growth performance of the enzymes phytase, xylanase, $\beta$-glucanase and their combinations were observed. Phytase enhanced the ADC of the P, Ca and ash fraction, besides that, the enzymes did not affect the digestibility. A comparison with previous studies using the same diet and enzyme treatments was made to elaborate on the discrepancy of the minimal effect of the enzyme supplementation in the present study. The response of fish fed the diet with enzymes (phytase and xylanase) was comparable in terms of FCR. The DM and NSP ADC was slightly higher in the present experiment, but not significantly different (figures 1 and 2). On the contrary, the control treatment without enzymes in the present study had a lower FCR and higher DM and NSP ADC, which were 
comparable to the diets supplemented with enzymes. Combining these studies suggest that over time, diet related factors affected the control diet and thus the repeatability of the study. It is likely that in the present study and other studies that do not show improvement in growth with the use of enzymes, the control diets were not sufficiently limiting in nutrients for growth as suggested by Adeola and Cowieson (2011).

\section{Acknowledgements}

We thank the staff of the aquaculture research facilities for their technical support in conducting the experiment and Ronald Booms and Tino Leffering for their assistance during laboratory work. This work is part of the Netherlands Organisation for Scientific Research (NWO) research programme "Green Aquafeeds" (project number 870.15.100). DuPont Animal Nutrition partly provided the enzymes, analysed the enzyme recovery and partly funded the project. 

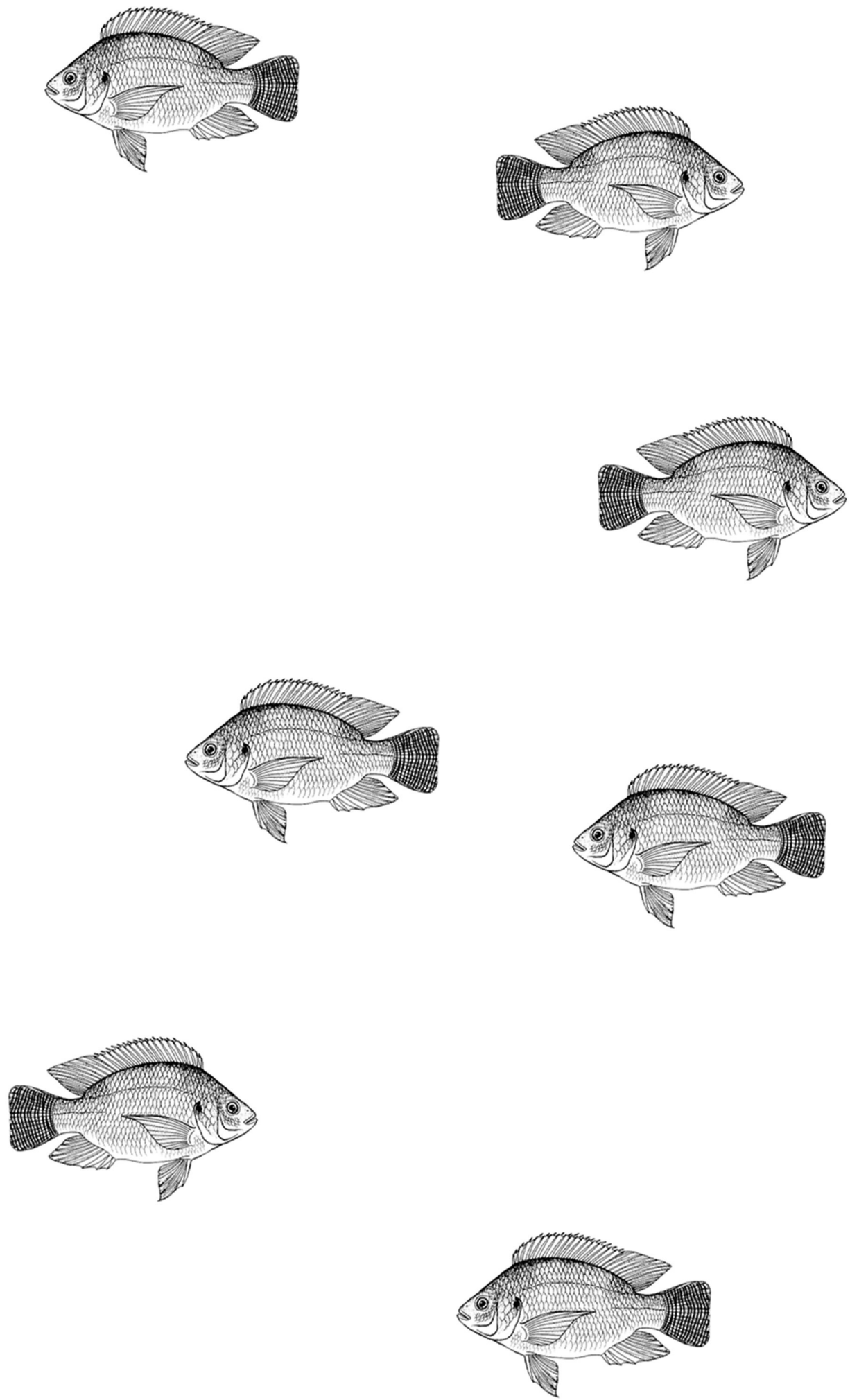


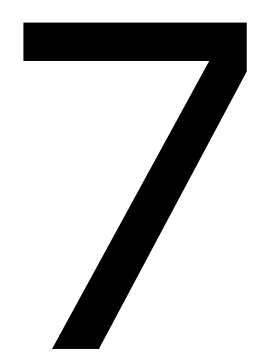

\title{
Effect of enzymes, probiotics and their combination on growth performance and nutrient utilisation in Nile tilapia
}

\author{
Roel M. Maas, Marc C.J. Verdegem, Sidup Debnath, \\ Leon Marchal, Johan W. Schrama
}

Aquaculture (2020), $533: 736226$ 


\section{Abstract}

The increased use of plant ingredients in aquafeeds over the last decades, as replacement for fish meal, has led to rising levels of undesired non-starch polysaccharides (NSP) and phytate. Both NSP and phytate degrading enzymes and probiotics have been widely studied. They can be used as a tool to deal with increasing levels of NSP and phytate in aquafeeds. However, studies combining both probiotics and enzymes are scarce in fish. The main objective of the present study was to assess the impact on Nile tilapia (Oreochromis niloticus) of enzymes and probiotics, as well as their synergistic effect. Parameters measured were: growth; nutrient digestibility; body composition; and the energy, nitrogen $(\mathrm{N})$, phosphorus $(P)$ and calcium balance. Diets were supplemented with, and without, an enzyme mix (phytase at $1000 \mathrm{FTU} / \mathrm{kg}$ and xylanase at $6000 \mathrm{U} / \mathrm{kg}$ ) and with, and without, probiotics (three strains of $B$. amyloliquefaciens at $60 \mathrm{mg} / \mathrm{kg}$ feed), according to a $2 \times 2$ factorial arrangement. This resulted in a control treatment (CON-CON) without enzymes and probiotics, an enzyme treatment (ENZ-CON), a probiotic treatment (CON-PRO) and a treatment with both enzymes and probiotics (ENZ-PRO). In total, 16 tanks (4 replicates/treatment) were used with 35 fish each (mean initial weight $39 \mathrm{~g}$ ). Fish were restrictively fed equal amounts of dry matter for 42 days. Both enzymes $(P<0.001)$ and probiotics $(P<0.05)$ improved growth $(\mathrm{g} / \mathrm{d})$ and FCR when applied individually. The combination of enzymes and probiotics showed an interaction effect $(P<0.05)$ on growth and FCR. Enzymes improved growth to a greater extent than probiotics, whereas the combination of enzymes and probiotics did not further enhance growth. The CON-CON treatment had the highest FCR (1.33), the CON-PRO treatment a slightly lower FCR (1.27); the lowest FCR (1.11) was found for both treatments with enzymes (ENZ-CON and ENZ-PRO). Enzyme supplementation improved the digestibility of all nutrients $(P<0.01)$, whereas probiotics enhanced fat digestibility $(P<0.01)$. Additionally, enzyme supplementation increased retained $P(\mathrm{mg} / \mathrm{d})$, retained $\mathrm{N}(\mathrm{mg} / \mathrm{d})$ and $\mathrm{N}$ efficiency $(P<0.001)$. Probiotic supplementation reduced the energy requirements for maintenance $\left(\mathrm{kJ} / \mathrm{kg}^{0.8} / \mathrm{d} ; P<0.05\right)$, resulting in numerically higher retained energy $(\mathrm{kJ} / \mathrm{d})$. Dietary supplementation of either enzymes or probiotics had positive effects on the measured parameters, but the combination of enzymes and probiotics did not have a synergistic effect. 


\section{Introduction}

As a finite resource, fishmeal is becoming increasingly unsustainable, from both an environmental and economic point of view. Thus, over recent decades, it has been increasingly replaced in aquafeeds by plant ingredients and the breadth of alternative ingredients is steadily growing (Bendiksen et al., 2011; Oliva-Teles et al., 2015; Tacon and Metian, 2015). High inclusion levels of plant ingredients coincide with antinutritional factors, of which non-starch polysaccharides (NSP) and phytate are probably the most prominent. NSP and phytate can negatively affect fish performance and health in various ways. Moreover, phytate and NSP are largely indigestible by fish due to the absence of the digestive enzymes required to break them down. Therefore, NSP and phytate have a low nutritional value, which limits the inclusion of plant ingredients (rich in NSP or phytate) in fish diets (Francis et al., 2001; Sinha et al., 2011; Kumar et al., 2012).

Tilapia is cultured in more than 100 countries and, in terms of production, it is the second most farmed fish in the world. Outside of Africa, most of the farmed tilapia belongs to the genus Oreochromis, of which Nile tilapia (Oreochromis niloticus) represents more than $90 \%$ (Wang and Lu, 2016). Tilapia are omnivorous and are capable of filter-feeding on phytoplankton, zooplankton and detritus (Garcia-Pérez et al., 2000; Azim et al., 2003). Tilapia is widely used as model species, including for studies on exogenous enzyme supplementation in fish diets. Carbohydrases, like xylanase, $\beta$-glucanase and cellulase, target NSP by hydrolysing the long polysaccharide chains into reduced sugars, which can provide energy through fermentation (Bergman, 1990; Bedford, 2000; Choct and Kocher, 2000). As a warm-water species with a long gut, the tilapia is well adapted for feeding on plant ingredients and has a high potential for fermentation in the gut. These factors make it a suitable fish for studies on the effect of exogenous enzyme supplementation (Metzler-Zebeli et al., 2010; Maas et al., 2020a). Indeed, many studies have been performed on tilapia, looking at how dietary carbohydrase enzymes can improve NSP digestibility (e.g., Lin et al., 2007; Li et al., 2009; Yigit and Olmez, 2011; Adeoye et al., 2016a; Hlophe-Ginindza et al., 2016; Maas et al., 2018; Hassaan et al., 2019; Maas et al., 2019).

In the last two decades, probiotics have appeared as an eco-friendly feed additive and alternative to antibiotics, in enhancing fish immunity and disease resistance. In addition, probiotics have been shown to improve nutrient utilisation and feed efficiency, resulting in improved growth. Probiotics alter the composition of the gut microbial community, increase the capacity to produce digestive enzymes (amylases, proteases, and lipases, etc.) and raise the supply of nutrients, like short-chain fatty acids (SCFA) and amino acids (Hai, 2015; Banerjee and Ray, 2017; Hoseinifar et al., 2017; Dawood et al., 2019; Kuebutornye et al., 2020). Many studies on probiotics (Bacillus) have been performed using tilapia (Aly et al., 2008a; Reda et al., 2016; Liu et al., 2017; Abarike et al., 2018), including a review by Hai (2015). 
Both NSP and phytate degrading enzymes and probiotics have been widely studied and can be used as a tool to deal with increasing levels of NSP, as well as to improve the sustainability of the aquaculture sector. However, studies combining probiotics and enzymes are scarce. The combination of enzymes and probiotics may result in a complementary mode of action. It is hypothesised that enzymes enhance the breakdown of NSP and stimulate the fermentation of NSP in the gut, producing SCFA. Probiotics (e.g. Bacillus spp.) may fare better in an SCFA enhanced gut, using the metabolites of NSP fermentation as an energy source. Vice versa, probiotics can alter the gut environment and are known to stimulate the activity of digestive enzymes, potentially favoring the breakdown and fermentation of NSP. In broilers, the synergy between probiotics and enzymes was tested in various studies (Seidavi et al., 2017; Wealleans et al., 2017; Konieczka et al., 2018). Konieczka et al. (2018) showed positive effects of interaction between enzymes (xylanase and $\beta$-glucanase) and probiotics (Bacillus subtilis) in terms of feed intake, (raised) concentrations of SCFA in the ileum, and (increased) bacterial enzyme activity in the caecal digesta. Wealleans et al. (2017) observed beneficial effects on nutrient digestibility and growth when enzymes (xylanase, amylase, and protease) and probiotics (3 strains of Bacillus amyloliquefaciens) were combined, suggesting a synergistic effect between enzymes and probiotics in broilers. To our knowledge, only Adeoye et al. (2016b) and Dai et al. (2019) tested the combined effect of enzymes and probiotics in fish. Adeoye et al. (2016b) focussed on the effects on growth, intestinal histology and microbiome in tilapia; whereas Dai et al. (2019) tested the effects on growth, digestive enzymes and the gut microbiome in snakehead. However, neither study tested for the interaction between enzymes and probiotics and a diet low in NSP was used. Therefore, in the present study we used a diet high in NSP for investigating the synergistic effect between dietary enzymes (phytase and xylanase) and probiotics (three strains of $B$. amyloliquefaciens) on growth, nutrient digestibility, body composition and the energy $(E)$, nitrogen $(N)$ and phosphorus $(P)$ balances in Nile tilapia.

\section{Materials and methods}

This study was approved by the Central Committee on Animal Experiments (CCD), under the advice of the Animal Experiment Committee (DEC) of The Netherlands (permit no. 2018.W-0010.002). It was also approved by the Ethical Committee for Animal Experiments of Wageningen University, The Netherlands, and carried out according to Dutch law (Act on Animal Experiments).

\section{Diets}

The effect of enzymes and probiotics was tested according to a $2 \times 2$ factorial arrangement. The first factor was supplementation with and without enzymes, using an enzyme mix of phytase (Axtra ${ }^{\circledR}$ PHY, Buttiauxella sp. phytase at 1000 FTU/kg, DuPont Animal Nutrition) and xylanase (Danisco ${ }^{\circledR}$ Xylanase at $6000 \mathrm{U} / \mathrm{kg}$, DuPont Animal Nutrition). The reason for using this enzyme mix is that the combination of phytase and xylanase is shown to be effective in Nile tilapia (Maas 
et al. 2018). The second factor was the incorporation with and without probiotics (Enviva ${ }^{\circledR}$ PRO 202 GT 60 mg kg feed providing 150,000 CFU/g feed, DuPont Animal Nutrition). Enviva ${ }^{\circledR}$ PRO 202 GT is a probiotic product that contains three strains of $B$. amyloliquefaciens spores, which are heat resistant. This resulted in a control treatment without enzymes and probiotics (CON-CON), a probiotic treatment (CON-PRO), an enzyme treatment (ENZ-CON) and a treatment with both enzymes and probiotics (ENZ-PRO). A basal diet was used for the incorporation of both enzymes and probiotics (Table 1). The basal diet was free of fish meal and formulated according to commonly applied levels of low quality ingredients, such as: wheat bran, rapeseed meal, sunflower meal, rice bran and wheat dried distillers grains with solubles. The choice of these low quality ingredients gave an approximate NSP level of $316 \mathrm{~g}$ per $\mathrm{kg}$ DM diet. This high NSP level was also used to make it a challenging diet, which may magnify the possible effect of the applied treatments. Extruded diets ( $3 \mathrm{~mm}$ pellets) were produced by SPAROS Lda. (Portugal). During extrusion, the temperature was kept below $110^{\circ} \mathrm{C}$. A batch of basal diet was extruded with and without probiotics. The enzymes (in liquid form), or placebo solution were mixed with the oils (fish, rapeseed and palm oil) and then vacuum coated at the research facilities of the Animal Science Group, Wageningen University, The Netherlands. The enzymes were diluted 1:50, with demineralised water, to increase the volume and to ensure a more homogenous dispersal of the enzymes during coating. The analysed colony-forming unit (CFU), enzyme recovery and nutrient composition are given in Table 2. Diets with (PRO-CON and PRO-ENZ) and without (CON-CON and CON-ENZ) probiotics had an average Bacillus colony-forming unit count of $7.9 \times 10^{4}$ and $3.0 \times 10^{3}$.

\section{Fish, rearing conditions and housing facilities}

The experiment was performed at the Aquaculture Research Facility (ARF) of Wageningen University, The Netherlands. Male Nile tilapia (Oreochromis niloticus; from the strain Silver $\mathrm{NMT}^{\mathrm{TM}}$ ) were obtained from a commercial fish breeder (TilAqua international, Someren, The Netherlands). Fish were housed in 120-L tanks and fed a commercial diet prior to the start of the experiment. During the experiment a total of 16 rectangular tanks of 60-L (effective volume) were used. The tanks were all connected to the same recirculation system, resulting in a common water supply and ensuring the same water quality for the inflow of each tank. The daily system water refreshment was 300-L. The recirculation system consisted of a sump, settling tank and trickling filter. Every single tank was connected to a swirl separator (AquaOptima AS, column height $44 \mathrm{~cm}$; diameter $24.5 \mathrm{~cm}$ ), with a detachable glass bottle at the bottom, to count feed spills and collect faeces for each tank separately. The water flow through each tank was set at $7 \mathrm{~L} / \mathrm{min}$, using a hand held liquid rotameter. All tanks were provided with a cylinder shaped air stone. The air stone and water flow ensured sufficient dissolved oxygen (DO). The photoperiod was set to $12 \mathrm{~h}$ light: $12 \mathrm{~h}$ dark (lights on 7:00, lights off 19:00). 
Table 1 ingredient composition of the basal diet (\%).

\begin{tabular}{lr}
\hline Diet & \\
\hline Ingredients (\%) & 7.0 \\
Maize & 10.0 \\
Soya bean meal & 6.88 \\
Wheat & 3.0 \\
Wheat gluten meal & 15.0 \\
Wheat bran & 10.0 \\
Rapeseed meal & 10.0 \\
Sunflower meal & 15.0 \\
Full-fat rice bran & 10.0 \\
Wheat DGGS & 1.0 \\
Fish oil & 1.5 \\
Rapeseed oil & 1.5 \\
Palm oil & 5.0 \\
Hydrolysed feathermeal & 1.0 \\
Mineral and vitamin premix & \\
Calcium carbonate $\left(\mathrm{CaCO}_{3}\right)$ & 0.7 \\
Dicalcium phosphate & 1.1 \\
DL-Methionine & 0.45 \\
L-Lysine & 0.65 \\
L-Threonine & 0.20 \\
Yttrium oxide & 0.02 \\
\hline
\end{tabular}

${ }^{+}$Dried Distillers Grain with Solubles.

‡ Premix composition (PVO 40/01, Sparos Lda, Olhão, Portugal). Vitamins (expressed as IU or mg/kg of final diet): vitamin A retinol acetate 20000; vitamin B1, 30; vitamin B2, 30; vitamin B3, 200; vitamin B5, 100; vitamin B6, 20; vitamin B12, 0.1; vitamin C, 1000; vitamin D3, 2000; vitamin E, 100; biotin, 0.3; inositol, 500; betaine, 500; choline chorine. Minerals (expressed as $\mathrm{mg} / \mathrm{kg}$ of final diet): copper sulphate, 9 ; ferric sulphate, 6 ; potassium iodide, 0.5 ; manganese oxide, 9.6; sodium selenite, 0.1 ; magnesium hydroxide, 7.5; calcium, 600; chlorine, 250.

Water quality parameters were measured three times per week (MondayWednesday-Friday) in the morning, before feeding, to ensure that the water quality parameters remained within the pre-set ranges. The average temperature was $27.5{ }^{\circ} \mathrm{C}( \pm 0.2)$. The $\mathrm{pH}$ range was set between 7.0 and 7.8 . Sodium bicarbonate was added to the system if the $\mathrm{pH}$ dropped below 7.0. The $\mathrm{pH}$ ranged between 6.8 and 7.9 during the experiment. DO levels of the common outflow stayed above $5.4 \mathrm{mg} / \mathrm{L}$. Conductivity was $8000 \mu \mathrm{S} / \mathrm{cm}$ at stocking, which was gradually lowered and kept around $3000 \mu \mathrm{S} / \mathrm{cm}$ after week one. The total ammonia level was $<0.25 \mathrm{mg} / \mathrm{L}$; nitrite $<0.15 \mathrm{mg} / \mathrm{L}$; and nitrate $<500 \mathrm{mg} / \mathrm{L}$. 
Table 2 analysed chemical composition, enzyme activity and bacillus count of the experimental treatments.

\begin{tabular}{|c|c|c|c|c|}
\hline \multirow[t]{2}{*}{ Treatment } & \multicolumn{2}{|c|}{ CON } & \multicolumn{2}{|c|}{ ENZ } \\
\hline & CON & PRO & CON & PRO \\
\hline \multicolumn{5}{|c|}{ Analysed nutrient content (g/kg DM) } \\
\hline Dry matter $(\mathrm{g} / \mathrm{kg})$ & 913 & 918 & 906 & 917 \\
\hline Crude protein & 316 & 313 & 312 & 312 \\
\hline Crude fat & 84 & 95 & 88 & 97 \\
\hline Total carbohydrates $^{+}$ & 529 & 519 & 528 & 519 \\
\hline Starch + sugars & 208 & 212 & 204 & 207 \\
\hline Non-starch polysaccharides ${ }^{\ddagger}$ & 321 & 308 & 323 & 311 \\
\hline Energy (kJ/g) & 20.7 & 20.7 & 20.8 & 20.7 \\
\hline Ash & 71 & 73 & 72 & 73 \\
\hline Phosphorus & 10.9 & 11.0 & 11.0 & 11.0 \\
\hline Calcium & 10.6 & 11.2 & 10.6 & 11.0 \\
\hline Magnesium & 3.74 & 3.72 & 3.74 & 3.69 \\
\hline Iron & 0.16 & 0.17 & 0.16 & 0.16 \\
\hline Manganese & 0.072 & 0.071 & 0.072 & 0.070 \\
\hline Zinc & 0.067 & 0.074 & 0.066 & 0.067 \\
\hline Copper & 0.021 & 0.22 & 0.021 & 0.022 \\
\hline Yttrium & 0.166 & 0.176 & 0.168 & 0.161 \\
\hline \multicolumn{5}{|l|}{ Enzyme activity } \\
\hline Phytase (FTU/kg) & $<180$ & $<180$ & 1127 & 1156 \\
\hline Xylanase (U/kg) & - & 386 & 6372 & 7620 \\
\hline \multicolumn{5}{|l|}{ Probiotics $^{\S}$} \\
\hline Bacillus count (CFU/g) & $2.8 \times 10^{3}$ & $8.5 \times 10^{4}$ & $3.2 \times 10^{3}$ & $7.3 \times 10^{4}$ \\
\hline
\end{tabular}

Notes. ENZ, enzyme supplementation; PRO, probiotic supplementation.

† The carbohydrate content (on DM basis) was calculated as: 1000 - (ash + crude protein + crude fat).

${ }^{\ddagger}$ Non-starch polysaccharides calculated as: carbohydrates - (starch + sugars).

$\S$ Enviva ${ }^{\circledR}$ PRO 202 GT (Three strains of B. amyloliquefaciens) incorporated at $60 \mathrm{mg} / \mathrm{kg}$ diet.

\section{Experimental procedure}

The experiment lasted 42 days. The four experimental treatments were assigned randomly to tanks. From a common batch, fish were caught and assigned to one of the 16 tanks at random. Fish were group weighted per tank, while mildly sedated using $(0.25 \mathrm{ml} / \mathrm{L})$ 2-phenoxyethanol. Each tank was stocked with 35 fish, having an average initial weight of $39 \mathrm{~g}$. At the end of the experiment all fish were batch weighed per tank and counted, while mildly sedated, to determine the final weight and calculate growth parameters. At the start of the experiment, 20 fish were euthanized using an overdose of 2-phenoxyethanol $(2 \mathrm{ml} / \mathrm{L})$, for initial body composition determination. At the end of the experimental period, 10 fish per tank were randomly selected and euthanized for final body composition. Fish samples were stored at $-20{ }^{\circ} \mathrm{C}$ until further analysis. 
The aim of the experiment was to test the effect of the added enzymes and probiotics on growth and nutrient utilisation. Therefore, the fish were fed a restricted equal amount of feed (dry matter (DM)) per tank per day. The feeding level was fixed and aimed at $16 \mathrm{~g} / \mathrm{kg}^{0.8}$ body weight $(\mathrm{BW}) / \mathrm{d}$, which is about $80 \%$ of the expected satiation level. The daily amount of feed was increased throughout the experiment by predicting fish growth and weight, using the average start weight of the fish and an expected feed conversion ratio (FCR) of 1.2. At the first feed, the fish were fed to $20 \%$ of the intended feeding level, which was increased over 6 days to $100 \%$. The daily feed ration was divided into two equal portions, fed at 9:00 and 15:30 h. The fish were hand fed and the duration of feeding did not exceed $1 \mathrm{~h}$ for all tanks.

Feed spills recovered from the settling units were recorded per tank after each feed. The diets were refrigerated $\left(4^{\circ} \mathrm{C}\right)$ throughout the experiment. Once a week, a sample of $100 \mathrm{~g}$ was taken from each diet. The feed samples were pooled per treatment and stored $\left(4{ }^{\circ} \mathrm{C}\right)$ until further analysis. Faeces were collected for digestibility studies, using swirl separators for 5 days per week (not the weekends), or until the tray was full for that week. The glass bottles were submerged in ice to prevent bacterial degradation of the faeces. Faeces were collected between the afternoon and morning feed (16:30 $\mathrm{h}-8: 00 \mathrm{~h})$. Faeces were pooled per week and stored in aluminium trays at $-20{ }^{\circ} \mathrm{C}$, until further analysis.

\section{Analyses}

Frozen fish samples $\left(-20{ }^{\circ} \mathrm{C}\right)$ were ground twice, using a meat mincer (Gastromaschinen, GmbH model TW-R 70; Feuma) with a $4.5 \mathrm{~mm}$ mesh, and homogenised. Fresh samples were taken for the determination of DM and crude protein (CP); samples for crude fat and energy were freeze dried prior to analysis. Faeces collected over the previous eight days were oven dried at $70{ }^{\circ} \mathrm{C}$. Feed, faeces and fish samples were analysed according to the same methods. DM content was determined by drying samples for at least $4 \mathrm{~h}$ at $103^{\circ} \mathrm{C}$, until constant weight (ISO 6496, 1983). Ash content was determined by incineration, using a muffle furnace for $4 \mathrm{~h}$ at $550{ }^{\circ} \mathrm{C}$ (ISO 5984, 1978). CP ( $\left.\mathrm{N} \times 6.25\right)$ was analysed using the Kjeldahl method (ISO 5983, 1979). Crude fat was measured by petroleum-ether extraction (Soxhlet method, ISO 5986). Energy content was measured using a bomb calorimetric, by direct combustion (IKA ${ }^{\circledR}$ werke, C7000; IKA analysentechnik, Weitershem, Germany). Yttrium, phosphorus (P), calcium (Ca) and magnesium $(\mathrm{Mg}$ ) were analysed in the feed and the faeces, using inductively coupled plasma-mass spectrometry (ICP-OES) according to the standard NEN 15510 (2007); iron (Fe), manganese (Mn), zinc ( $\mathrm{Zn})$ and copper (Cu) were analysed in the feed only.

The phytase and xylanase activity in feed samples was analysed by DuPont Innovation Laboratories (Brabrand, Denmark). Phytase was analysed using the methods described by Yu et al. (2012). One phytase unit (FTU) was defined as the amount of enzyme required to release $1 \mu \mathrm{mol}$ of inorganic $P$ per minute from 
sodium phytate at $\mathrm{pH} 5.5$ and $37^{\circ} \mathrm{C}$. Xylanase was analysed using the methods described by Romero et al. (2013). One xylanase unit (U) was defined as the amount of enzyme that releases $0.48 \mu \mathrm{mol}$ of reducing sugar as xylose, from wheat arabinoxylan, per minute at $\mathrm{pH} 4.2$ and $50{ }^{\circ} \mathrm{C}$. The probiotics count (CFU) of spore forming Bacillus was enumerated using tryptone soy agar (DuPont in house method, Wilmington, USA).

\section{Calculations}

The growth (in $\mathrm{g} / \mathrm{d}$ ) was calculated as the difference between the average individual initial weight $\left(\mathrm{W}_{\mathrm{i}}\right)$ and the final $\left(\mathrm{W}_{\mathrm{f}}\right)$ body weight, per tank, divided by the duration ( $t$ ) of the experiment in days (d). The specific growth rate (SGR in \% body weight /d) was calculated as $\left(\ln \left(W_{f}\right)-\ln \left(W_{i}\right)\right) \times 100 / t$. The daily absolute feed intake (in $\mathrm{g} \mathrm{DM} / \mathrm{d}$ ) was calculated as $\mathrm{FI}_{\text {tot }} /(\mathrm{n} \times \mathrm{t}$ ), where $\mathrm{n}$ is the number of fish per tank and $\mathrm{FI}_{\text {tot }}$ is the total feed intake (in $\mathrm{g} D M$ ), corrected for dead fish and feed spills. The geometric mean body weight $\left(W_{G}\right.$ in $g$ ) was calculated as $\sqrt{ }\left(W_{f} \times\right.$ $\mathrm{W}_{\mathrm{i}}$ ) and the mean metabolic body weight (MBW in $\mathrm{kg}^{0.8}$ ) as $\left(\mathrm{W}_{\mathrm{G}} / 1000\right)^{0.8}$. The FCR was calculated as daily absolute feed intake / growth. The survival of fish per tank was calculated as $(\mathrm{Nf} / \mathrm{Ni}) \times 100$, where $\mathrm{Nf}$ is the final number of fish and $\mathrm{Ni}$ the initial number.

Yttrium was used as an inert marker to calculate the apparent digestibility coefficient (ADC in \%) of DM, crude protein, crude fat, total carbohydrate, starch, NSP, gross energy, ash, $\mathrm{P}, \mathrm{Ca}$ and $\mathrm{Mg}$ for each tank. The ADC was calculated as (Cheng and Hardy, 2002): ADC (\%) = $100 \times\left[1-\left(Y_{i} \times\right.\right.$ amount nutrient in faeces) / $\left(Y_{f} X\right.$ amount nutrient in feed)], where $Y$ is the concentration of yttrium in the diet and faeces (in $\mathrm{g} / \mathrm{kg} \mathrm{DM}$ ) and $\mathrm{N}$ is the quantity of nutrients (in $\mathrm{g} / \mathrm{kg} \mathrm{DM}$ ), or energy content (in $\mathrm{kJ} / \mathrm{g} D M$ ), in the diet and faeces. The total amount of carbohydrates $(\mathrm{g} / \mathrm{kg})$ in feed and faeces was calculated on a DM basis as: $1000-$ (crude protein + crude fat + ash). The NSP fraction was calculated as: total carbohydrates - (starch + sugars).

The energy $(E \mathrm{~kJ} / \mathrm{d})$, nitrogen $(\mathrm{N} \mathrm{mg} / \mathrm{d}), \mathrm{P}$ and $\mathrm{Ca}(\mathrm{mg} / \mathrm{d})$ balance parameters were calculated per tank and expressed on a per fish basis. The parameters were calculated as described by Saravanan et al. (2012). Generally, balance parameters are expressed in relation to metabolic body weight. However in the present study, fish were fed an equal amount of feed (on DM) of identical nutrient composition, and thus with similar amounts of N, E, P and Ca. The final weights of the fish were affected by enzyme and probiotic supplementation (see result), therefore using metabolic body weight would already create differences in balance parameters, due to the differences in final body weight.

For the $\mathrm{N}$ balance, $\mathrm{N}$ intake was calculated as: the product of feed intake and dietary $\mathrm{N}$ content; digestible $\mathrm{N}$ intake as $\mathrm{N}$ intake times the digestibility coefficient of $\mathrm{N}$; retained $\mathrm{N}$ as the difference between final and initial $\mathrm{N}$ body mass; branchial urinary $\mathrm{N}$ losses as the digestible $\mathrm{N}$ intake minus retained $\mathrm{N}$. The $\mathrm{N}$ efficiency was calculated as retained $\mathrm{N}$ (RN) divided by digestible $\mathrm{N}(\mathrm{DN})$. P and Ca balances were 
calculated according to the same principle as the $\mathrm{N}$ balance. For the $\mathrm{E}$ balance, $\mathrm{E}$ intake was calculated as: the product of feed intake and dietary $E$ content; digestible $E(D E)$ intake as $E$ intake times the $E$ digestibility coefficient; brachial and urinary $\mathrm{E}$ losses as branchial and urinary $\mathrm{N}$ losses times the $\mathrm{E}$ content of $\mathrm{NH}_{3}-$ $\mathrm{N}(24.9 \mathrm{~kJ} \mathrm{~N} / \mathrm{g})$, assuming that all $\mathrm{N}$ was excreted as $\mathrm{NH}_{3}-\mathrm{N}$; metabolisable $\mathrm{E}$ intake as DE minus branchial and urinary $E$; Retained $E(R E)$ as the difference between final and initial body energy content; and heat production as metabolisable $E$ minus RE. The $E$ requirements for maintenance (MEm) were calculated from the metabolisable $E$ (ME) and the $E$ retained as protein (REp) and fat (REf). The following formula was used to calculate the metabolisable E: MEm = ME - ( REp / $0.5)+(\operatorname{REf} / 0.9))$. In this calculation, an energetic utilisation efficiency of metabolisable $E$ for protein gain of $50 \%$ was assumed; similarly an energetic utilisation efficiency of metabolisable $E$ for fat gain of $90 \%$ was assumed (Lupatsch et al., 2003).

\section{Statistics}

For all statistical analysis, the tank was considered as the experimental unit. A two-way ANOVA was used to test for significance of the effects of enzyme and probiotic supplementation (and their combined effect), for all data. When an interaction effect was detected $(P<0.05)$, a Tukey HSD (honest significant difference) test was conducted (with multiple comparisons and $95 \%$ level of significance), to compare treatment means. All data were expressed as mean per treatment of four replicates. All statistical analyses were carried out using the IBM Statistical Package for the Social Sciences (SPSS) ( 2 25.0; New York, NY, USA).

\section{Results}

\section{Performance}

Survival was high (>99.3\%) and unaffected by the treatment (Table 3 ). The initial weight $(39 \mathrm{~g})$ was similar between treatments $(P>0.1)$. Conforming to the experimental design, absolute feed intake was identical among treatments (1.69 $\mathrm{g}$ DM/d). Enzyme supplementation (phytase and xylanase) increased growth rate $(\mathrm{g} / \mathrm{d})$, specific growth rate (SGR \%/d) and decreased FCR $(P<0.001$; Table 3$)$. Addition of probiotics increased the growth rate and decreased FCR $(P<0.05)$, and tended to improve SGR $(P<0.1)$. For all performance traits (growth, SGR and FCR), there was an interaction effect between enzyme and probiotics $(P<0.05)$. Enzymes improved the growth rate to a greater extent than probiotics, whereas the combination of enzymes and probiotics did not further enhance growth. The CONCON treatment had the highest FCR (1.33), followed by the CON-PRO treatment, which had a significantly lower FCR (1.25). The lowest FCR (1.11) was found for those treatments containing either enzymes or a combination of enzymes and probiotics. 
Table 3 Effect of enzyme and probiotic supplementation on the performance of Nile tilapia over 42 days.

\begin{tabular}{|c|c|c|c|c|c|c|c|c|}
\hline \multirow[t]{2}{*}{ Treatment } & \multicolumn{2}{|c|}{$\mathrm{CON}$} & \multicolumn{2}{|c|}{ ENZ } & \multirow[b]{2}{*}{ SEM } & \multicolumn{3}{|c|}{$P$-values } \\
\hline & CON & PRO & CON & PRO & & ENZ & PRO & ENZ*PRO \\
\hline Survival (\%) & 99.3 & 97.9 & 100.0 & 100.0 & 1.19 & ns & ns & ns \\
\hline Initial body weight (g) & 38.9 & 39.1 & 38.9 & 39.2 & 0.26 & ns & ns & ns \\
\hline Final body weight (g) & $92.1^{\mathrm{a}}$ & $95.5^{\mathrm{b}}$ & $102.8^{c}$ & $103.2^{c}$ & 0.78 & $* * *$ & $*$ & $\#$ \\
\hline Feed intake (g DM/d) & 1.69 & 1.69 & 1.69 & 1.69 & 0.00 & - & - & - \\
\hline \multicolumn{9}{|l|}{ Growth } \\
\hline Growth $(\mathrm{g} / \mathrm{d})$ & $1.27^{\mathrm{a}}$ & $1.34^{\mathrm{b}}$ & $1.52^{\mathrm{c}}$ & $1.52^{c}$ & 0.015 & $* * *$ & $*$ & $*$ \\
\hline $\operatorname{SGR}(\% / d)$ & $2.05^{\mathrm{a}}$ & $2.12^{\mathrm{b}}$ & $2.31^{\mathrm{c}}$ & $2.31^{c}$ & 0.015 & $* * *$ & $\#$ & $*$ \\
\hline FCR & $1.33^{\mathrm{a}}$ & $1.25^{\mathrm{b}}$ & $1.11^{\mathrm{c}}$ & $1.11^{\mathrm{c}}$ & 0.014 & $* * *$ & $*$ & $*$ \\
\hline
\end{tabular}

Notes. ENZ, enzyme (effect) supplementation; PRO, probiotic (effect) supplementation; ENZ*PRO, interaction effect; SGR, specific growth rate; FCR, feed conversion ratio. Values are means and standard error of the mean (SEM). Means within the same row not sharing a common letter are significantly different $(P<0.05)$; ns, not significant, $P>0.1$; \#, tendency $P<0.1 ; *, P<0.05$; ***, $P<0.001$.

\section{Digestibility}

The apparent digestibility coefficients (ADC) of all nutrients, were enhanced for diets supplemented with enzymes (Table 4). Enzyme supplementation had a large impact on ash digestibility and mineral availability; ADC of Ash, P, Ca and Mg were increased by 48, 51, 135 and 35\%, respectively. Probiotic supplementation reduced crude protein digestibility $(P<0.05)$ and tended to reduce NSP ADC $(P=0.084)$, from $33.5 \%$ to $30.5 \%$. Diets supplemented with enzymes (ENZ-CON and ENZ-PRO) had a higher NSP ADC, compared to diets without enzymes (CONCON and CON-PRO) (36.1\% versus $27.9 \% ; P<0.001)$. Probiotic supplementation improved fat ADC $(P<0.05)$, but this effect was influenced by enzyme supplementation (interaction; $P<0.05$ ). The improvement in fat ADC by probiotics was stronger in the absence of enzymes. The ADC of fat improved from $87.1 \%$, for the CON-CON treatment, to $91.6 \%$, for the ENZ-PRO treatment. Ca ADC was also affected by an interaction between enzymes and probiotics $(P<0.05)$. Probiotic supplementation increased $\mathrm{Ca} A D C$ for treatments without enzyme supplementation, but decreased $\mathrm{Ca} A D C$ in treatments with enzyme supplementation (Table 4).

\section{Body composition}

Body protein content $(\mathrm{g} / \mathrm{kg})$ was unaffected by the dietary treatments $(P>0.1$; Table 5). Enzyme supplementation reduced the body fat content by $11 \mathrm{~g} / \mathrm{kg}$ fresh weight $(P<0.001)$. This corresponds to a lower energy content $(P<0.01)$, and a tendency towards a lower DM content, for fish fed diets containing enzymes. Enzyme supplementation had a large impact on the body mineral content. Ash, $\mathrm{P}$, $\mathrm{Ca}$ and $\mathrm{Mg}$ levels were higher in fish fed diets with enzymes $(P<0.001)$. Compared to the initial body composition, ash and mineral contents were higher in fish fed 
diets with enzymes, whereas ash and mineral content was lower in fish fed diets without enzymes. Probiotic supplementation resulted in a higher body ash content $(P<0.05)$, although numerically the difference was small. Probiotic supplementation tended to increase body $P$ content $(P=0.055)$. There was no interaction effect between enzymes and probiotics on body composition parameters $(P>0.1$; Table 5$)$.

Table 4 Effect of enzyme and probiotic supplementation on nutrient digestibility (ADC, \%) of Nile tilapia over 42 days.

\begin{tabular}{|c|c|c|c|c|c|c|c|c|}
\hline \multirow[t]{2}{*}{ Treatment } & \multicolumn{2}{|c|}{ CON } & \multicolumn{2}{|c|}{ ENZ } & \multirow[b]{2}{*}{ SEM } & \multicolumn{3}{|c|}{$P$-values } \\
\hline & $\mathrm{CON}$ & PRO & CON & PRO & & ENZ & PRO & ENZ*PRO \\
\hline \multicolumn{9}{|l|}{$A D C(\%)$} \\
\hline Dry matter & 67.5 & 68.2 & 72.3 & 71.3 & 0.88 & $* * *$ & ns & ns \\
\hline Crude protein & 89.1 & 89.0 & 89.7 & 89.4 & 0.11 & $* * *$ & $*$ & $\#$ \\
\hline Crude fat & $87.1^{\mathrm{a}}$ & $89.8^{\mathrm{b}}$ & $91.0^{\mathrm{bc}}$ & $91.6^{\mathrm{c}}$ & 0.57 & $* * *$ & $* *$ & * \\
\hline Total carbohydrates & 55.9 & 56.1 & 61.7 & 59.5 & 1.39 & $* *$ & ns & ns \\
\hline Starch & 98.2 & 98.1 & 98.5 & 98.3 & 0.14 & $* *$ & ns & ns \\
\hline NSP & 28.6 & 27.3 & 38.4 & 33.6 & 2.22 & $* * *$ & $\#$ & ns \\
\hline Energy & 72.4 & 72.9 & 75.9 & 74.9 & 0.74 & $* * *$ & ns & ns \\
\hline Ash & 34.0 & 36.5 & 52.1 & 51.1 & 1.49 & $* * *$ & ns & ns \\
\hline Phosphorus & 41.2 & 44.4 & 64.8 & 64.2 & 1.51 & $* * *$ & ns & ns \\
\hline Calcium & $14.4^{\mathrm{a}}$ & $20.9^{a}$ & $42.5^{b}$ & $40.4^{b}$ & 2.66 & $* * *$ & ns & $*$ \\
\hline Magnesium & 46.0 & 48.4 & 63.8 & 63.4 & 1.52 & $* * *$ & ns & ns \\
\hline
\end{tabular}

Notes. ENZ, enzyme (effect) supplementation; PRO, probiotic (effect) supplementation; ENZ*PRO, interaction effect; ns, not significant, $P>0.1$; \#, tendency, $P<0.1 ; *, P<0.05 ; * *, P<0.01$; ***, $P<0.001$; NSP, non-starch polysaccharides; Values are means and standard error of the mean (SEM). Means within the same row not sharing a common letter are significantly different $(P<0.05)$.

Table 5 Effect of enzymes and probiotic supplementation on body composition (on fresh weight basis, $\mathrm{g} / \mathrm{kg}$ ) of Nile tilapia over 42 days.

\begin{tabular}{|c|c|c|c|c|c|c|c|c|c|}
\hline \multirow[t]{2}{*}{ Treatment } & \multirow[b]{2}{*}{ Initial } & \multicolumn{2}{|c|}{ CON } & \multicolumn{2}{|c|}{ ENZ } & \multirow[b]{2}{*}{ SEM } & \multicolumn{3}{|c|}{$P$-values } \\
\hline & & $\mathrm{CON}$ & PRO & CON & PRO & & ENZ & PRO & ENZ*PRO \\
\hline \multicolumn{10}{|l|}{ Unit in $\mathrm{g} / \mathrm{kg}$ fresh } \\
\hline Dry matter & 273 & 284 & 285 & 280 & 279 & 2.5 & $\#$ & ns & ns \\
\hline Crude protein & 152 & 152 & 152 & 153 & 151 & 1.6 & ns & ns & ns \\
\hline Crude fat & 85 & 99 & 98 & 89 & 86 & 2.1 & $* * *$ & ns & ns \\
\hline Energy $(\mathrm{kJ} / \mathrm{g})$ & 6.9 & 7.5 & 7.6 & 7.1 & 7.0 & 0.12 & $* *$ & ns & ns \\
\hline Ash & 32.7 & 28.2 & 28.8 & 34.5 & 35.6 & 0.35 & $* * *$ & $*$ & ns \\
\hline Phosphorus & 5.4 & 4.6 & 4.7 & 5.8 & 6.0 & 0.07 & $* * *$ & $\#$ & ns \\
\hline Calcium & 8.6 & 7.1 & 7.4 & 9.6 & 9.7 & 0.15 & $* * *$ & ns & ns \\
\hline Magnesium & 0.30 & 0.27 & 0.27 & 0.31 & 0.32 & 0.005 & $* * *$ & ns & ns \\
\hline
\end{tabular}

Notes. Initial, initial body composition; ENZ, enzyme (effect) supplementation; PRO, probiotic (effect) supplementation; ENZ*PRO, interaction effect; ns, not significant, $P>0.1$; \#, tendency $P<0.1 ; *, P<0.05 ; * *, P<0.01 ; * * *, P<0.001$. Values are means and standard error of the mean (SEM). 


\section{Balances}

The nitrogen $(N)$, energy $(E)$ phosphorus $(P)$ and Calcium (Ca) balances, expressed on an individual fish basis as $\mathrm{mg} / \mathrm{d}$ or $\mathrm{kJ} / \mathrm{d}$, are displayed in Table 6 . Parallel to the protein digestibility, probiotic supplementation affected the DN intake negatively $(P<0.001)$. Besides that, probiotic supplementation did not affect the $\mathrm{N}$ balance $(P>0.1)$. Enzyme supplementation reduced branchial and urinary $\mathrm{N}$ losses $(P>0.001)$ and increased RN $(P>0.001)$. This pattern of reduced branchial and urinary $\mathrm{N}$ loses and increased RN, was reflected by significantly higher $(17 \%) \mathrm{N}$ efficiency in diets containing enzymes $(P<0.001)$. N efficiency was comparable for the ENZ-CON and ENZ-PRO treatments (49.5\% and 48.9\%); the CON-PRO treatments had a lower $\mathrm{N}$ efficiency (43.6\%); and the treatments without enzymes or probiotics (CON-CON) had the lowest $N$ efficiency $(40.7 \%)$. There was a tendency for an interaction effect of enzymes and probiotics on the RN $(P=0.099)$. It was observed that probiotic supplementation increased RN only in treatments without enzyme supplementation ( 30.9 vs. $32.9 \mathrm{mg} / \mathrm{d}$ ), not in treatments with enzymes (37.5 vs. $36.7 \mathrm{mg} / \mathrm{d}$ ).

Enzyme supplementation reduced branchial and urinary $\mathrm{E}$ loss $(P<0.01)$ and increased metabolisable $\mathrm{E}$ intake $(P<0.001)$. In line with a higher metabolisable $\mathrm{E}$ intake, there was a tendency for higher heat $E(P=0.076)$ and $R E(P=0.061)$ with enzyme supplementation. Similar to RN, $E$ retained as protein (RN multiplied by $23.7 \mathrm{~kJ} / \mathrm{g}$ ) was higher for fish fed diets with enzymes, compared to fish fed diets without enzymes. In contrast, $\mathrm{E}$ retained as fat was unaffected by any of the dietary treatments $(P>0.1)$. In diets supplemented with enzymes, increased $\mathrm{E}$ retained as protein, and unchanged $E$ retained as fat, resulted in leaner fish. Expressed in $\mathrm{kJ} / \mathrm{kg}^{0.8} / \mathrm{d}$, MEm was reduced with probiotic supplementation $(P<0.05)$. Averaged over probiotic treatments, MEm for treatments with and without probiotics was 75.5 and $82.6 \mathrm{~kJ} / \mathrm{kg}^{0.8} / \mathrm{d}$, respectively (Table 6 ). The energy efficiency (RE/DE) was unaffected by the dietary treatments $(P>0.1)$, ranging between 39.4 and $42.3 \%$.

Parallel to $P$ digestibility, the dietary $P$ intake (DP) increased $(P<0.05)$ by $51 \%$ with enzyme supplementation. Despite the higher DP intake, branchial and urinary $P$ loss was unaffected by the dietary treatments $(P>0.1)$. Both enzyme and probiotic supplementation enhanced retained $\mathrm{P}(\mathrm{RP})(P<0.01)$; the largest improvement was demonstrated with enzyme supplementation. Enzyme supplementation enhanced the RP (averaged over the control treatments) from $5.5 \mathrm{mg} / \mathrm{d}$ (CONCON and CON-PRO treatments) to $9.4 \mathrm{mg} / \mathrm{d}$ (ENZ-CON and ENZ-PRO treatments); whereas probiotic supplementation enhanced the RP (averaged over the enzyme treatments) from $7.2 \mathrm{mg} / \mathrm{d}$ (CON-CON and ENZ-CON treatments) to $7.7 \mathrm{mg} / \mathrm{d}$ (CON-PRO and ENZ-PRO treatments). The $P$ efficiency (RP/DP) was affected by enzyme supplementation $(P<0.001)$. The lowest $P$ efficiency was found with the CON-CON treatment $(67.5 \%)$, followed by the CON-PRO $(70.3 \%)$, ENZ-CON $(76.8 \%)$ and ENZ-PRO (80.9\%) treatments. P balance traits were not affected by an interaction effect between enzymes and probiotics $(P>0.05)$. 
Table 6 Effect of enzyme and probiotic supplementation on nitrogen, energy, phosphorous and calcium balances of Nile tilapia over 42 days.

\begin{tabular}{|c|c|c|c|c|c|c|c|c|}
\hline \multirow[t]{2}{*}{ Treatment } & \multicolumn{2}{|c|}{ CON } & \multicolumn{2}{|c|}{ ENZ } & \multirow[b]{2}{*}{ SEM } & \multicolumn{3}{|c|}{$P$-values } \\
\hline & CON & PRO & CON & PRO & & ENZ & PRO & ENZ*PRO \\
\hline \multicolumn{9}{|l|}{ Nitrogen balance $(\mathrm{mg} / \mathrm{d})$} \\
\hline Gross N intake & 85.3 & 84.7 & 84.5 & 84.1 & 0.000 & - & - & - \\
\hline Digestible $\mathrm{N}$ intake & 76.0 & 75.4 & 75.9 & 75.1 & 0.068 & $* *$ & $* * *$ & ns \\
\hline Branchial and urinary $\mathrm{N}$ loss & 45.0 & 42.5 & 38.3 & 38.3 & 0.735 & $* * *$ & ns & ns \\
\hline $\mathrm{N}$ retention & 30.9 & 32.9 & 37.5 & 36.7 & 0.749 & $* * *$ & ns & \# \\
\hline $\mathrm{N}$ efficiency (RN/DN) & 40.7 & 43.6 & 49.5 & 48.9 & 0.983 & $* * *$ & ns & ns \\
\hline \multicolumn{9}{|l|}{ Energy Balance $(\mathrm{kJ} / \mathrm{d})$} \\
\hline Gross E intake & 35.0 & 35.0 & 35.2 & 34.9 & 0.013 & - & - & - \\
\hline Digestible E intake & 25.3 & 25.5 & 26.7 & 26.1 & 0.184 & $* * *$ & ns & $\#$ \\
\hline Branchial and urinary E loss & 1.1 & 1.1 & 1.0 & 1.0 & 0.023 & $* *$ & ns & ns \\
\hline Metabolisable E & 24.2 & 24.4 & 25.7 & 25.2 & 0.194 & $* * *$ & ns & \# \\
\hline Heat $\mathrm{E}$ & 14.2 & 13.6 & 14.8 & 14.3 & 0.321 & $\#$ & ns & ns \\
\hline Retained E & 10.0 & 10.8 & 10.9 & 10.9 & 0.261 & $\#$ & ns & ns \\
\hline Retained $\mathrm{E}$ as protein & 4.6 & 4.8 & 5.5 & 5.4 & 0.111 & $* * *$ & ns & ns \\
\hline Retained $\mathrm{E}$ as fat & 5.4 & 5.9 & 5.4 & 5.4 & 0.261 & ns & ns & ns \\
\hline E maintenance & 9.1 & 8.1 & 8.7 & 8.3 & 0.338 & ns & $\#$ & ns \\
\hline E maintenance $\left(\mathrm{kJ} / \mathrm{kg}^{0.8} / \mathrm{d}\right)$ & 86.3 & 76.0 & 78.9 & 75.0 & 2.895 & ns & $*$ & ns \\
\hline E efficiency (RE/DE, \%) & 39.4 & 42.3 & 41.0 & 41.6 & 1.024 & ns & ns & ns \\
\hline \multicolumn{9}{|l|}{ Phosphorus balance $(\mathrm{mg} / \mathrm{d})$} \\
\hline Gross $\mathrm{P}$ intake & 18.4 & 18.6 & 18.6 & 18.5 & 0.013 & - & - & - \\
\hline Digestible P intake & 7.6 & 8.3 & 12.0 & 11.9 & 0.199 & $* * *$ & ns & $\#$ \\
\hline Branchial and urinary $\mathrm{P}$ loss & 2.5 & 2.5 & 2.8 & 2.3 & 0.239 & ns & ns & ns \\
\hline $\mathrm{P}$ retention & 5.1 & 5.8 & 9.3 & 9.6 & 0.164 & $* * *$ & $* *$ & ns \\
\hline $\mathrm{P}$ efficiency (RP/DP, \%) & 67.5 & 70.3 & 76.8 & 80.9 & 1.929 & $* * *$ & ns & ns \\
\hline \multicolumn{9}{|l|}{ Calcium balance $(\mathrm{mg} / \mathrm{d})$} \\
\hline Gross Ca intake & 18.4 & 18.6 & 18.6 & 18.5 & 0.013 & - & - & - \\
\hline Digestible $\mathrm{Ca}$ intake & $2.7^{\mathrm{a}}$ & $3.9^{\mathrm{a}}$ & $7.9^{b}$ & $7.5^{\mathrm{b}}$ & 0.354 & $* * *$ & ns & $*$ \\
\hline Branchial and urinary Ca loss & -5.1 & -5.0 & -7.6 & -8.3 & 0.437 & $* * *$ & ns & ns \\
\hline Ca retention & 7.7 & 8.9 & 15.5 & 15.8 & 0.338 & $* * *$ & $\#$ & ns \\
\hline Ca efficiency (RCa/DCa, \%) & 310 & 234 & 197 & 212 & 25.124 & $*$ & ns & \# \\
\hline
\end{tabular}

Notes. ENZ, enzyme (effect) supplementation; PRO, probiotic (effect) supplementation; ENZ*PRO, interaction effect; ns, not significant, $P<0.1$; \#, tendency, $P<0.1 ; *, P<0.05 ; * *, P<0.01 ; * * *$, $P<0.001 ; \mathrm{RN}$, retained nitrogen; $\mathrm{DN}$, digestible nitrogen; RE, retained energy; $\mathrm{DE}$, digestible energy; $\mathrm{RP}$, retained phosphorous; $\mathrm{DP}$, digestible phosphorous; $\mathrm{RCa}$, retained calcium; $\mathrm{DCa}$, digestible calcium. Values are means and standard error of the mean (SEM). Means within the same row not sharing a common letter are significantly different $(P<0.05)$.

Enzyme supplementation affected all Ca balance traits $(P<0.05$; Table 6$)$. Retained $\mathrm{Ca}$ (RCa) for all treatments was higher than the digestible Ca intake (DCa), indicating water-borne Ca uptake, as shown by the negative branchial and urinary Ca losses. Enzyme supplementation enhanced the RCa from $8.3 \mathrm{mg} / \mathrm{d}$ (averaged over the control treatments: CON-CON and CON-PRO) to $15.7 \mathrm{mg} / \mathrm{d}$ (averaged 
over the enzyme treatments: ENZ-CON and ENZ-PRO); whereas probiotic supplementation had a tendency $(P=0.057)$ to increase the RCa. An increase in $\mathrm{Ca}$ efficiency with decreasing levels of DCa can be observed. This suggests that for the $\mathrm{RCa}$, the relative contribution of $\mathrm{Ca}$ uptake from the water increased with decreasing levels of DCa.

\section{Discussion}

Dietary supplementation of enzymes (phytase and xylanase), as well as probiotics (three strains of $B$. amyloliquefaciens), enhanced growth performance of Nile tilapia (Table 3). Previous studies, using the same enzymes (xylanase and phytase; but including other dietary enzymes) (Lin et al., 2007; Adeoye et al., 2016a; Maas et al., 2018; Maas et al., 2019) and various probiotics (Aly et al., 2008a; Aly et al., 2008b; Wang et al., 2008; Ayyat et al., 2014; Reda and Selim, 2015; Saputra et al., 2016; Abarike et al., 2018) also observed improvements in growth performance of Nile tilapia. In the present study, the FCR of the CON-CON treatment (without enzymes and probiotics) was high (1.33); fish of a comparable size normally have a FCR of around 0.90 (Amirkolaie et al., 2006; Saravanan et al., 2012; Adeoye et al., 2016b; Maas et al., 2018). This high FCR with the CONCON treatment can be attributed to the higher dietary NSP level (i.e. lower diet quality), which resulted in a lower dry matter digestibility (67.5\%; Table 4) and thus lower growth rate (Sinha et al., 2011; Maas et al., 2020b).

One of the objectives of this study was to explore the synergy between enzyme and probiotic supplementation. The positive effect of both enzymes and probiotics did not result in a synergistic effect on growth. In the present study, the improvement in growth was greater with enzyme supplementation (FCR with ENZCON treatment: 1.11 ) than with probiotics (FCR with CON-PRO treatment: 1.25), compared to the control treatment (FCR with CON-CON treatment: 1.33). The combination of enzymes and probiotics (ENZ-PRO) did not further enhance growth, compared to the enzyme supplemented treatment (ENZ-CON), but maintained the same values for the growth parameters. It was expected that the combination could show an additive or synergistic effect, however this was not observed in this study. Compared to the control treatment (CON-CON), the enzymes alone (ENZCON) enhanced the growth rate $(\mathrm{g} / \mathrm{d})$ by approximately $20 \%$, when feeding the same diet at the same feeding level $(\mathrm{g} / \mathrm{d})$. This effect the enzymes (ENZ-CON) had on the growth rate could have resulted in realising the maximum growth potential of the diet. A study by Adeoye et al. (2016b) also tested the combined effect of enzymes and probiotics; they found the improvement on growth to be minimal. This could partly be due to the use of a commercial diet, which alone (without enzymes and probiotics) already had a good FCR (0.94). A high nutritional value of the control diet, as in the study of Adeoye et al. (2016b), or a large improvement in nutritional value through enzyme supplementation (present study), may limit any further improvement in diet quality through additional additives, such as other enzymes and probiotics (Cowieson and Bedford, 2009; Maas et al., 2019). In 
addition, many studies on broilers showed that the effect of probiotics is more effective under challenging conditions (Cao et al., 2013; Zhang et al., 2016). It could be expected that under increasingly challenging conditions (i.e. a commercial setting), the combination could lead to better growth performance. Although the probiotics did not further enhance growth performance in the presence of enzymes, the postulated effects probiotics have, like improvements in immune response, disease resistance and feed intake (Newaj-Fyzul et al., 2014; Hai, 2015; Kuebutornye et al., 2020), remain plausible, as this was not investigated in the present study. In the study of Adeoye et al. (2016b), the effect of probiotics on the intestinal morphology was maintained in the presence of enzymes. Therefore it cannot be concluded, from the present data, whether or not the use of probiotics, in the presence of enzymes, can have positive impacts, such as improvements in gut health.

Like the growth parameters, there were no synergistic effects between enzyme and probiotic supplementation on digestibility. The tendency of probiotics to reduce NSP digestibility (Table 4) could have contributed to lowered nutrient ADCs. The combination of enzymes and probiotics (ENZ-PRO) resulted in the highest fat digestibility $(91.6 \%)$, although not significantly higher compared to the treatment with only enzymes (ENZ-CON; $91.0 \%$ ). In this study, fish fed the control treatment (CON-CON) displayed a NSP digestibility of $28.6 \%$. That tilapia can endogenously digest NSP, is in line with recent meta-analysis across studies, where an average NSP digestibility for tilapia of $24.3 \%$ was reported (Maas et al., 2020a). Supplementation with phytase and xylanase significantly increased the NSP digestibility by approximately 29\%. Likewise, Maas et al. (2018) and Maas et al. (2019) showed that the combination of phytase and xylanase can increase NSP digestibility. Contrary to the effect of the enzymes, the probiotics had a tendency to reduce NSP digestibility, from $33.5 \%$ (average CON-CON and ENZ-CON) to $30.5 \%$ (average PRO-CON and PRO-ENZ). To the best of our knowledge, effects of probiotics on NSP digestibility have not previously been reported in fish. Probiotics (B. amyloliquefaciens) are known to affect the gut in various ways (physiology, gut microbiota, production of metabolites, $\mathrm{pH}$, etc.), altering the gut environment (Hai, 2015; Dai et al., 2019; Dawood et al., 2019). Changes in the gut environment may have led to less favourable conditions for the supplemented enzymes (phytase and xylanase) and endogenous digestive enzymes, thereby reducing NSP digestibility. It has been shown that the NSP level can affect the digestibility of nutrients other than NSP (Haidar et al., 2016; Maas et al., 2018; Maas et al., 2020b). NSP are known to have various antinutritional effects (affecting gut physiology, morphology etc.), which can directly or indirectly contribute to reduced nutrient digestibility (Choct and Kocher, 2000; Sinha et al., 2011). In contrast to the higher NSP digestibility of the enzyme treatment (ENZCON), our findings indicate that the negative effect of NSP on nutrient digestibility is likely increased with combined enzyme-probiotic supplementation (ENZ-PRO treatment), as shown by a decrease in the ADC. In addition, enzymes had a strong effect on nutrient digestibility and retention of $\mathrm{N}$, energy and $\mathrm{P}$, which were all 
numerically higher for the enzyme treatment (ENZ-CON), compared to the probiotic treatment (CON-PRO). If enzymes and probiotics compete for the same substrate, this might explain the lack of additivity.

Although probiotics did not further enhance the growth rate in the presence of enzymes, when supplemented on their own (CON-PRO), probiotics increased the growth rate by approximately $5.5 \%$, compared to the control treatment (CONCON). The basal diets already contained large amounts of spore forming Bacillus, however the inclusion of $B$. amyloliquefaciens still improved growth performance. The improvement in growth rate for the probiotic treatment (CON-PRO) is best explained by looking at the $\mathrm{N}$ and energy balances (Table 6). The probiotics improved fat digestibility by 2.7 percentage points for the CON-PRO treatment, compared to the CON-CON treatment. However, probiotics did not enhance nutrient digestibility. Probiotics are known to produce digestive enzymes and to stimulate enzyme activity (amylases, proteases, lipases, etc.), which can result in enhanced nutrient digestibility (Chen et al., 2016; Dawood et al., 2019; Makled et al., 2019; Kuebutornye et al., 2020). In the present study, it is notable that probiotic supplementation resulted in a strong increase in fat digestibility, whilst digestibility of other nutrients did not increase. Protein digestibility was actually negatively affected by the presence of probiotics. This suggests that a factor other than the production of digestive enzymes/stimulation of enzyme activity, is responsible for the increase in fat digestibility, for instance, changes in fat emulsification or chyme characteristics. The average energy requirement for maintenance (MEm) was $79.1 \pm 4.4 \mathrm{~kJ} / \mathrm{kg}^{0.8} / \mathrm{d}$, which is considerably higher than estimated by some other studies (Lupatsch et al., 2010; Schrama et al., 2012; Maas et al., 2018), where the MEm ranged between 54.7 and $64.1 \mathrm{~kJ} / \mathrm{kg}^{0.8} / \mathrm{d}$. Saravanan et al. (2013) showed that the diet can have a strong effect on the MEm, when the dietary electrolyte balance disturbs the acid-base homeostasis, resulting in an increase in MEm from 57 to $88 \mathrm{~kJ} / \mathrm{kg}^{0.8} / \mathrm{d}$. In the study by Haidar et al. (2016), the average estimated MEm was high $\left(103 \mathrm{~kJ} / \mathrm{kg}^{0.8} / \mathrm{d}\right)$; here an increase in dietary NSP level increased the MEm by $14 \%$, suggesting that the energy cost of digestion increases with higher levels of NSP (and its subsequent effect on the intestinal microbial balance). In the present study, probiotics reduced the MEm by approximately $9.4 \%$ (from 82.6 to $75.5 \mathrm{~kJ} / \mathrm{kg}^{0.8} / \mathrm{d}$ ); even though enzymes reduced the MEm by approximately $5 \%$ (from 81.3 to $76.9 \mathrm{~kJ} / \mathrm{kg}^{0.8} / \mathrm{d}$ ), this was not statistically significant. Haidar et al. (2016) suggested that NSP digestibility could result in an increased cost of digestion, thereby increasing the MEm. In the present study, probiotic supplementation had a tendency to reduce NSP digestibility (Table 4), whilst enzyme supplementation increased NSP digestibility. However, both enzymes and probiotics lowered the MEm, compared to the control treatment (CON-CON), with the lowest MEm occurring with the combination of enzymes and probiotics ( 75.0 vs $86.3 \mathrm{~kJ} / \mathrm{kg}^{0.8} / \mathrm{d}$ ). This suggests that something else, other than NSP digestibility, affects the MEm, such as the gut microbiota, gut health or the gut barrier function. Most aspects of the gut barrier function are actively controlled, but suboptimal conditions, like a poor quality diet, can disturb the gut barrier 
function (Jutfelt, 2011). Both enzymes (through the production of volatile fatty acids etc.) and probiotics can affect the gut microbiota, as well as the gut barrier function (Balcázar et al., 2007; Nayak, 2010; Ige, 2013; Zhou et al., 2013), and subsequently maintenance. The lower MEm, with diets supplemented with probiotics, meant more energy was available for growth. The probiotic treatment (CON-PRO) retained about $8 \%$ more energy, compared to fish on the control treatment (CON-CON), however this was not statistically significant. The retained $\mathrm{E}(\mathrm{kJ} / \mathrm{d})$ was highly comparable between the CON-PRO (10.8), ENZ-CON (10.9) and ENZ-PRO (10.9) treatments. Although the digestible $\mathrm{N}$ intake was numerically lower for the probiotic treatment (CON-PRO), compared to the control treatment (CON-CON), the retained $\mathrm{N}$, and thus the $\mathrm{N}$ efficiency, was higher (numerically) for the probiotic treatment (43.6\%) compared to the control treatment $(40.7 \%)$. Likewise, both El-Haroun et al. (2006) and Makled et al. (2019) showed an improved protein efficiency ratio (PER) in Nile tilapia, when feeding diets supplemented with probiotics.

Besides the significant effect of enzymes on nutrient digestibility, both the $\mathrm{N}$ retention and $\mathrm{N}$ efficiency were increased, by approximately $20 \%$, for the treatments containing enzymes (ENZ-CON and ENZ-PRO), compared to the control treatment (CON-CON) (Table 6). This significant effect of enzymes on the $\mathrm{N}$ balance is in accordance with the substantial improvement $(+20 \%)$ in growth rate (Table 3). As $\mathrm{N}$ retention comes simultaneously with water gain, the effect of increasing $\mathrm{N}$ retention is large. This explains the $13 \%$ higher growth rate with enzyme supplemented diets, compared to diets supplemented with probiotics (CON-PRO), despite the comparable energy retention. An increase in $\mathrm{N}$ retention and efficiency, with the use of enzymes (phytase and xylanase), was previously observed in Maas et al. (2018), however the cause of this increased $\mathrm{N}$ efficiency is unclear.

It is uncertain from the results, whether the available dietary $P$ levels were sufficient to sustain maximal body $P$ content. The amount of $P$ in the diets was 11 $\mathrm{g} / \mathrm{kg}$ DM feed, which is considerably higher than the recommended level of 4.0 $\mathrm{g} / \mathrm{kg}$ (according to the NRC, 2011). The diets were formulated to have an available $P$ level of $4.7 \mathrm{~g} / \mathrm{kg}$ DM feed. This was to meet the $P$ requirements for growth and for attaining maximal whole-body $P$ concentration, in accordance with metaanalysis across fish species (excluding Rainbow trout) by Prabhu et al. (2013). In this study, the calculated available $P(g / k g D M)$ (using Table 2 and 5 ) for the different treatments was: 4.5 (CON-CON), 4.9 (CON-PRO), 7.1 (ENZ-CON) and 7.1 (ENZ-PRO). The $P$ availability with the enzyme supplemented treatments was higher, subsequently both the $P$ retention (absolute, $\mathrm{mg} / \mathrm{d}$; Table 6 ), as well as the body $P$ content $(\mathrm{g} / \mathrm{kg}$; Table 5$)$, increased. This indicates that the available $P$ levels in the diets without enzymes were too low to sustain maximal whole body $P$ concentrations. The relationship between available dietary $P$ levels ( $g / k g ~ D M)$ and whole-body $P$ content ( $\mathrm{g} / \mathrm{kg}$ live weight) was extrapolated according to a linear broken line model, by Prabhu et al. (2013). Prabhu et al. (2013) suggest that, above the level required to sustain maximal whole $\mathrm{P}$ concentrations, $\mathrm{P}$ will be 
excreted (branchial and urinary $\mathrm{P}$ loss) thereby reducing the $\mathrm{P}$ efficiency (retained $\mathrm{P} /$ digestible $\mathrm{P}$ ). However, in the present study, fish retained relatively more $\mathrm{P}$ with increasing available $P$ levels, resulting in a higher $P$ efficiency. The average $P$ efficiency for the enzyme treatments was $78.9 \%$, in comparison to $68.9 \%$ for the treatments without enzymes (Table 6). It cannot be speculated whether the available $P$ levels, in the enzyme treatments, were above the level required to sustain maximal body $P$ content. Maas et al. (2018) found that the P efficiency stayed rather constant with increasing available $\mathrm{P}$ levels (using phytase), showing a high average value of $92 \%$.

To summarize, both enzymes and probiotics enhanced growth performance, with the largest improvement due to enzymes. The enzymes improved nutrient digestibility of all nutrients, whereas probiotics enhanced fat digestibility. Besides affecting nutrient digestibility, enzymes stimulated a significant increase in retained $\mathrm{N}$ and $\mathrm{P}$, and $\mathrm{N}$ efficiency. Probiotics reduced the energy requirements for maintenance, thereby increasing retained energy (numerically). Individually, both enzymes and probiotics had positive effects on the measured parameters, however a synergistic or complementary mode of action between enzymes and probiotics was not observed in this study.

\section{Acknowledgements}

We thank the staff of the aquaculture research facilities for their technical support in conducting the experiment and Ronald Booms and Tino Leffering for their assistance during laboratory work. This work is part of the Netherlands Organisation for Scientific Research (NWO) research programme "Green aquafeeds" (project number 870.15.100). DuPont Animal Nutrition provided the enzymes and probiotics, analysed the enzyme recovery and colony-forming unit of the probiotics, and partly funded the NWO project. 

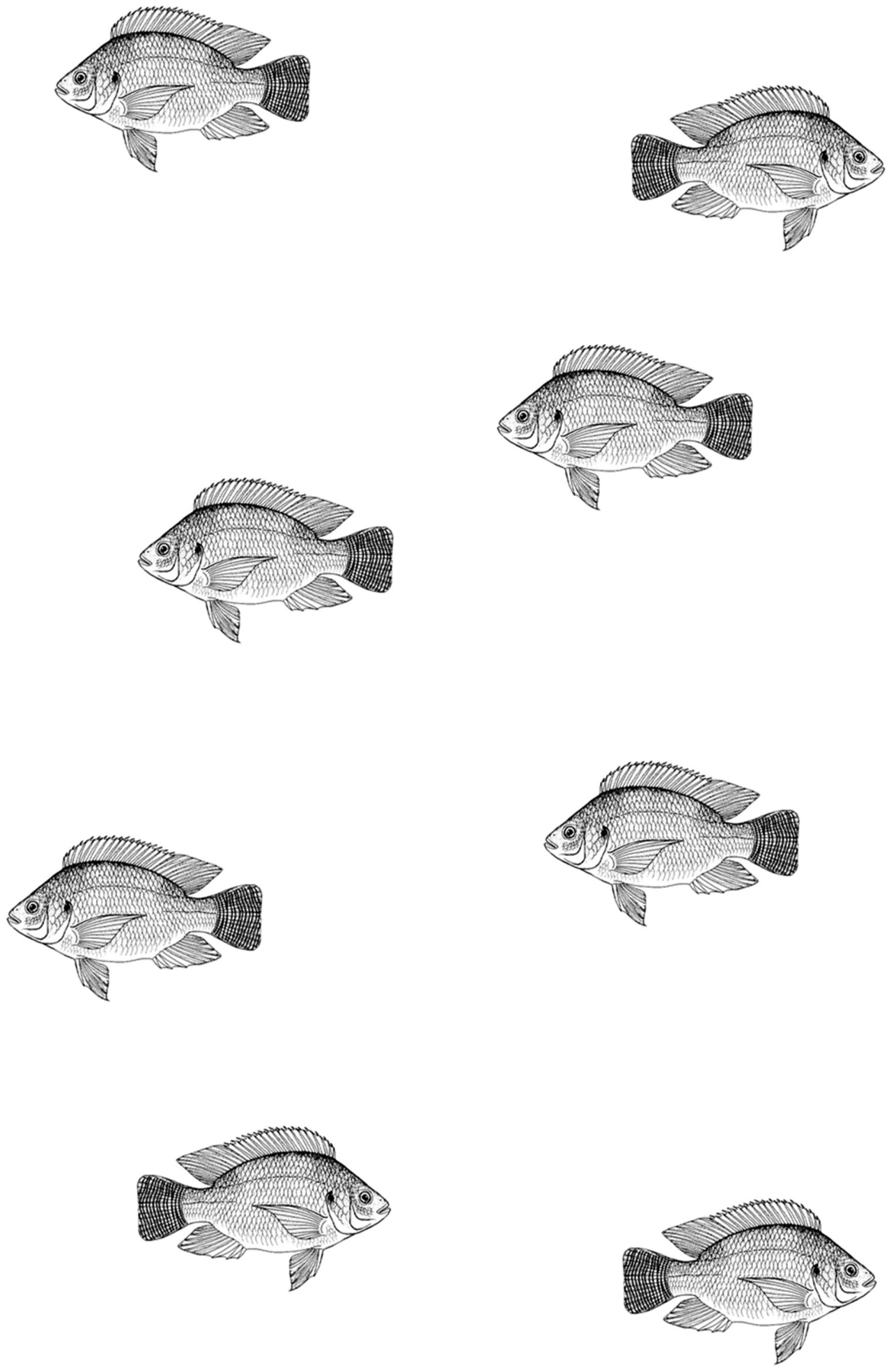


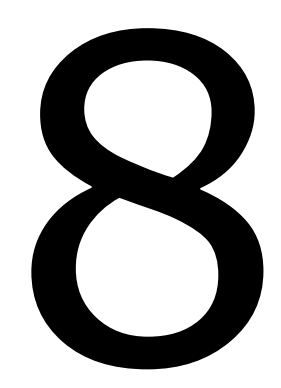

\section{Exogenous enzymes and probiotics alter digestion kinetics and microbial interactions in Nile tilapia gut}

Roel M. Maas*, Yale Deng*, Yeuming Dersjant-Li, Jules Petit,

Marc C.J. Verdegem, Johan W. Schrama, Fotini Kokou

* These authors contributed equally to this work

Submitted for publication 


\section{Abstract}

Sustainable aquafeed production requires fishmeal replacement, leading to an increasing use and inclusion of plant-derived ingredients. As a consequence, higher levels of antinutritional substances, such as non-starch polysaccharides and phytate, are present in aquafeeds, with negative effects on fish performance, nutrient digestibility and overall gut health. To alleviate these negative effects, providing exogenous digestive enzymes and/or probiotics can be an effective solution. In this study, we tested the effect of dietary supplementation of exogenous enzymes (phytase and xylanase), probiotics (three strains of Bacillus amyloliquefaciens) and their interactions, on nutrient digestion kinetics and volatile fatty acid (VFA) content along the gastrointestinal tract, as well as the gut microbiome diversity in Nile tilapia. Four diets were formulated, including a control diet without enzyme or probiotic supplementation (CON-CON), a diet with enzymes (ENZ-CON), a diet with probiotics (CON-PRO) and a diet with both enzymes and probiotics (ENZ-PRO). Chyme total VFA content (acetic, propionic, (iso) butyric, (iso) valeric acids) was not influenced by enzymes, but increased with probiotic supplementation in the proximal gut. The lactate concentration decreased only with enzyme supplementation along the gastrointestinal tract. Enzyme supplementation affected the location of crude protein, calcium ( $\mathrm{Ca}$ ) and phosphorus $(P)$ digestibility; crude protein digestibility was higher in the proximal and middle intestine, while $\mathrm{P}$ and $\mathrm{Ca}$ digestibility were enhanced from respectively proximal and middle intestine onward. The microbial diversity in the distal gut was not affected by either enzyme or probiotic supplementation. A dominant microbial community was detected, composed of five species, related to carbohydrate fermentation. Supplementation of enzymes and probiotics enhanced species interactions as shown by network analysis, while it also increased the abundance of lactic acid bacteria (Lactobacillales order) and Bacillus species. Such results suggest that dietary supplementation with exogenous enzymes and probiotics increases nutrient availability, while on the same time benefits gut health and contributes to a more stable microbiome environment. 


\section{Introduction}

Aquaculture is one of the fastest-growing food production sectors providing more than half of the fish supply around the world (FAO, 2020). To meet the increasing demand for feeds while ensuring a sustainable growth for aquaculture, there is a trend over the past decades to switch from fisheries-dependent fish meal as the main protein ingredient to widely-available plant-based ingredients (Tacon and Metian, 2015). However, commonly used plant-based ingredients such as soybean, rapeseed and sunflower meals, contain a wide variety of antinutritional factors that impair fish performance (Francis et al., 2001). Non-starch polysaccharides (NSP), protease inhibitors and phytic acid (i.e., phytate) are antinutritional factors reported to negatively affect nutrient digestibility and mineral absorption ( $\mathrm{Fe}, \mathrm{Mg}, \mathrm{Zn}, \mathrm{Cu}, \mathrm{Ca}$ and $\mathrm{P}$ ), reducing the nutrient utilisation efficiency and fish growth (Francis et al., 2001; Sinha et al., 2011). Consequently, to be able to efficiently use plant-based ingredients in the fish feeds, it is important to alleviate their antinutritional effects. For instance, phytate bound $\mathrm{P}$ generally remains unavailable for fish, because of the lack of endogenous enzymes to break down phytate. Inorganic $P$ needs to be supplemented in aquafeeds that contain high levels of plan-based ingredients in order to fulfil the $P$ requirements, this is undesired as mined $P$ is exhaustible. Improving the bioavailability of phytate bound $P$ with phytase, reduces the dependency of aquaculture on inorganic $P$ sources (Prabhu et al., 2013; Obersteiner et al., 2013).

As monogastric animals, fish lack NSP-degrading enzymes such as $\beta$-glucanases and $\beta$-xylanases, that allow digestion of the long polysaccharide chains present in NSP-rich plant-based ingredients, and therefore lack the ability to efficiently utilize nutrients from plant-based diets (Maas et al., 2020a). Commercially, to increase the nutrient availability and digestibility of the feeds, exogenous enzymes, such as phytase and xylanases, are commonly applied in the animal feed formulations, that catalyze the hydrolysis of phytic acid and polymeric carbohydrates, respectively (Adeola and Cowieson, 2011). Addition of phytase and $\beta$-xylanase was reported to improve nutrient digestibility and growth in several fish species (Castillo and Gatlin, 2015; Lemos and Tacon, 2017; Maas et al., 2018; Maas et al., 2019; Zheng et al., 2020). The digestion of NSP in the gastrointestinal tract (GI-tract) is associated with fermentation by commensal microbes, producing beneficial volatile fatty acids (VFA), which are rapidly absorbed by the intestine (Collinder et al., 2009; Sinha et al., 2011). The type of carbohydrates in the diet can have significant effects on the composition of the intestinal microbiota and consequently on the type and amount of VFAs produced (Abdel-Latif et al., 2020). The principle VFAs produced in the GI-tract of monogastric terrestrial animals are acetate, propionate and butyrate, and at low concentrations also formate, valerate, caproate, isobutyrate and isovalerate (Williams et al., 2001). In the fish GI-tract, the principle VFA produced is acetate, while propionate and butyrate are produced at lower levels (Maas et al., 2020a). Levels of lactic acid, which is commonly found in the GI-tract of monogastric animals like pigs (Argenzio et al., 1974), have not been reported in fish, although an in vitro study by Leenhouwers et al. (2008) 
showed a potential of lactic acid production by inoculum collected from the GItract of Nile tilapia (Oreochromis niloticus).

Dietary supplementation with probiotics is reported to have positive effects on fish health and disease resistance. Recent studies also showed the dietary probiotics can enhance the nutrient digestibility of aquatic animals by increasing digestive enzymes activity (Hoseinifar et al., 2017). Among the probiotic candidates, Bacillus species have been widely used in aquaculture, due to their sporulation capacity and positive effects on feed utilisation, immune response (Kuebutornye et al., 2019) and digestive enzyme activity such as protease, amylase, trypsin, and lipase (Hamza et al., 2016; Hauville et al., 2016). Besides, supplementation of some Bacillus species through water bath or live feed was shown to modulate the gut microbial composition in fish (Giatsis et al., 2016; Jiang et al., 2019). Studies in aquatic and livestock animals reported that probiotics may affect the gut microbiome structure by steering microbial interactions, potentially promoting intestinal homeostasis (Yang et al., 2017; Ma et al., 2018). However, it remains unclear whether Bacillus species can improve the utilisation efficiency of plantbased diets and how they may influence microbial structure in the fish GI-tract.

Nile tilapia is the third most important aquaculture species by volume (FAO, 2020), with omnivorous dietary habits and the potential for GI-tract fermentation. Supplementing plant-based diets with phytase and $\beta$-xylanases improved growth and digestibility of dry matter (DM), crude protein (CP), carbohydrate and ash, showing a good potential for application in Nile tilapia (Maas et al., 2018; Maas et al., 2019). Several studies have been performed so far using Bacillus strains as probiotics in Nile tilapia diets, however, focusing mainly on the effects on immune response and growth performance (Ridha and Azad, 2012; Selim and Reda, 2015; Silva et al., 2015). To our knowledge, no studies so far have focused on either the impact of exogenous enzymes or probiotics and their combination on the kinetics of macro- and micronutrient digestion and VFA production along the GI-tract of Nile tilapia. The combined supplementation of exogenous enzymes and probiotic could result in a complimentary mode of action. The ability to produce digestive enzymes (including NSP-degrading enzymes) by the probiotic may complement the endogenous enzyme activity. Moreover, the exogenous enzymes may increase the availability of suitable substrate for the probiotic as well as may promote the growth of beneficial bacteria in the GI-tract. The aim of this study was to improve the nutritional utilisation efficiency of plant-based diets for Nile tilapia by increasing NSP digestibility and digestive enzyme activity or the gut microbiome structure. For this purpose, we assessed the effect of dietary supplementation of an enzymatic cocktail (phytase and $\beta$-xylanase) and a cocktail of three probiotic Bacillus strains on (1) the kinetics of the digestion of DM, CP and minerals of the diet; (2) the VFA and lactate concentrations along the GI-tract; and (3) the gut microbiome structure of Nile tilapia. This was experimentally tested in a two-bytwo factorial design to quantify the effects of enzyme and probiotic addition (factors), and their interactions. 


\section{Materials and methods}

This study was approved by the Central Committee on Animal Experiments (CCD), under the advice of the Animal Experiment Committee (DEC) of The Netherlands (permit no. 2018.W-0010.002). It was also approved by the Ethical Committee for Animal Experiments of Wageningen University, The Netherlands, and carried out according to Dutch law (Act on Animal Experiments).

\section{Experimental design and animal husbandry}

The effect of the dietary supplementation of enzymes and probiotics was tested according to a $2 \times 2$ factorial arrangement, with 4 replicates per treatment. The first factor compared supplementation versus no supplementation with enzymes, using an enzyme cocktail consisting of phytase (Axtra ${ }^{\circledR}$ PHY, Buttiauxella sp. phytase at $1000 \mathrm{FTU} / \mathrm{kg}$, DuPont Animal Nutrition) and xylanase (Danisco ${ }^{\circledR}$ Xylanase at $6000 \mathrm{U} / \mathrm{kg}$, DuPont Animal Nutrition). The second factor compared supplementation versus no supplementation with probiotic mix (Enviva ${ }^{\circledR}$ PRO 202 GT, three strains of $B$. amyloliquefaciens at $60 \mathrm{mg} / \mathrm{kg}$ feed). This resulted in: a control diet without enzyme and probiotics (CON-CON), a diet with only the probiotic mix (CON-PRO), a diet with only the enzyme cocktail (ENZ-CON), and a diet with the enzyme cocktail and the probiotic mix (ENZ-PRO). Extruded diets (3 $\mathrm{mm}$ pellets) were produced by SPAROS Lda. (Portugal). The basal diet was free of fish meal and formulated with current commonly applied low quality ingredients, rich in dietary NSP (see chapter 7). Two batches of feed were extruded, with and without the probiotic mix, using heat resistant spores (powder form). Diets with (PRO-CON and PRO-ENZ) and without (CON-CON and CON-ENZ) probiotics had an average measured colony-forming unit (CFU) counts of $7.9 \times 10^{4}$ and $3.0 \times 10^{3}$, respectively. After extrusion, the oils and enzymes in liquid form were coated together onto the pellets under vacuum at the research facilities of the Animal Science Group, Wageningen University, The Netherlands.

This experiment consisted of two periods: the first period was a balance period (day 0-42) to determine the faecal nutrient digestibility, nutrient balances and fish growth performance (discussed in chapter 7); during the second period (day 4347) digesta and mucus were collected along the gastrointestinal tract to determine the VFA content and kinetics of digestion, as well as the gut microbiome composition, respectively (discussed in present chapter). The experiment was performed at the Aquaculture Research Facility (ARF) of the Wageningen University, The Netherlands. Male Nile tilapia (Oreochromis niloticus; from the strain Silver $\mathrm{NMT}^{\mathrm{TM}}$ ) were obtained from a commercial fish breeder (Til-Aqua international, Someren, The Netherlands). The fish were fed daily $16 \mathrm{~g}$ feed $/ \mathrm{kg}^{0.8} / \mathrm{d}$ which corresponds to approximately $80 \%$ of expected satiation feeding, to assure all the feed was eaten in all tanks. The daily feed ration was divided into two equal portions fed at 9:00 and 15:30 h. During the experiment, 16 rectangular tanks with an effective volume of 60-L were used. Each tank was stocked with 35 fish with the average body weight of $39 \mathrm{~g}$. All tanks were connected to the same 
common water supply as part of one recirculating aquaculture system (RAS). The RAS water treatment section included a sump, a solid removal unit and a biofilter. Each tank was connected to a swirl separator from AquaOptima AS (internal diameter of $24.5 \mathrm{~cm}$ and $44 \mathrm{~cm}$ column height) to collect faeces. Each swirl separator had a detachable glass bottle to collect faeces and uneaten pellets connected at the bottom outlet. Water flow per tank was maintained at $7 \mathrm{~L} / \mathrm{min}$ during the experiment. Each tank contained an air stone. The photoperiod was 12 $\mathrm{h}$ light: $12 \mathrm{~h}$ dark, switching the light on at $7.00 \mathrm{am}$. Water quality parameters were monitored three times a week, before the first feeding. The water temperature was maintained at $27.5{ }^{\circ} \mathrm{C}( \pm 0.2)$ and $\mathrm{pH}$ ranged between 7.0 and 7.9. DO level in water inlet to the solid removal unit (common outflow of tanks) in the RAS never dropped below $5.4 \mathrm{mg} / \mathrm{L}$. At stocking, the conductivity was 5000 $\mu \mathrm{S} / \mathrm{cm}$, and was gradually declined to $4000-3000 \mu \mathrm{S} / \mathrm{cm}$ at the end of the first week. Total ammonia nitrogen, nitrite- $\mathrm{N}$ and nitrate- $\mathrm{N}$ during the experiment, remained below than $0.25,0.15$ and $500 \mathrm{mg} / \mathrm{L}$, respectively.

\section{Sampling procedure}

Samples for body composition and gut microbiota were taken at the end of the balance period (period 1), these fish were $24 \mathrm{~h}$ deprived of feed prior to sampling. All other samples were taken at the end of period 2 on fed fish.

For gut microbiota, 3 fish per tank were randomly selected, euthanized with an overdose of 2-phenoxyethanol ( $2 \mathrm{ml} / \mathrm{L})$ and weighted. Fish were disinfected with $70 \%$ ethanol before dissection. The distal gut was separated from the rest of the gut and divided into two by length, and the first $5 \mathrm{~cm}$ in the direction of the anus was sampled. The collected samples were transferred to cryotubes and submerged in liquid nitrogen before storing at $-80{ }^{\circ} \mathrm{C}$ until further analysis for gut microbiome.

Fish not taken for body composition and microbiota at the end of the balance period, were continued to be fed for 3 or 4 days using approximately a $10 \%$ higher feeding ration as the balance period using a belt feeder (feeding $24 \mathrm{~h}$ ). This was done in order to ensure that fish were full so that digesta could be collected along the gastrointestinal tract (GI-tract). On day 46 and 47, eight tanks (2 replicates per treatments per sampling day) were sampled and all fish were euthanized by an over-dosed of 2-phenoxyethanol ( $3 \mathrm{ml} / \mathrm{L}$ ) for digesta collection. In each tank, feeding was stopped approximately $1 \mathrm{~h}$ before sampling the fish, to prevent collection of whole pellets in the stomach. Samples of digesta were collected in four sections of the GI-tract: stomach and proximal, middle, and distal part of the intestine. The proximal part was taken from the stomach (after the pyloric part of the stomach) until the spiral part of the intestine (gut becomes thinner), the division of the middle and distal part was done based on having equal lengths per section. Digesta per section was pooled per tank and collected in pre-weighted crucibles. Crucibles and fish not directly sampled were kept on ice at all time to stop bacterial activity and prevent degradation of the faeces. Digesta was collected for DM, CP, ash, P, Ca, Cu, Mg, Mn, Fe, Zn and VFA measurements. VFAs were 
measured in the digesta as an indication for fermentation in the GI-tract. Freshly collected digesta $(0.5-\mathrm{ml})$ was added to $0.5-\mathrm{ml}$ of buffer $(1-\mathrm{ml}$ distilled water and $50-\mu$ phosporic acid) with iso-caproic acid as internal standard in cryotubes and stored at $-20{ }^{\circ} \mathrm{C}$ until analysis. For the analyses of lactic acid, 1-ml of digesta (in duplicate) was collected and stored (no buffer) in cryotubes at $-20{ }^{\circ} \mathrm{C}$. The crucibles with the remaining digesta were weighted to determine the DM content of the digesta in the different segments of the GI-tract and dried at $70{ }^{\circ} \mathrm{C}$ until further analysis.

\section{VFA and lactic acid content along the gastrointestinal tract}

Samples stored for VFA analyses $\left(-20^{\circ} \mathrm{C}\right)$ were thawed at room temperature, mixed (vortex mixer) and centrifuged for $10 \mathrm{~min}$ at $10.000 \mathrm{rpm}$. Supernatant was taken and put in 2-ml clear glass vails with insert and sealed using aluminum caps with silicone septa. The concentrations of acetic, propionic, iso-butyric, butyric, iso-valeric and valeric acid were measured as previously described (Qaisrani et al., 2014); VFA were separated by gas chromatography using a HP-FFAP (30 $\mathrm{m} \times 0.32$ $\mathrm{mm}, 0.25 \mu \mathrm{m}$ ) column from Agilent (Santa Clara, California, USA) and hydrogen as the mobile phase with detection by flame ionization detector. Quantification of VFA was based on a chemical standard solution (Merck, Hohenbrunn, Germany) after internal standard correction. VFA concentrations were expressed in $\mathrm{mmol}$ per $L$ of fresh digesta.

For the lactate content measurement, digesta samples stored at $-20{ }^{\circ} \mathrm{C}$ were thaw at room temperature. Prior to analysis, digesta were centrifuged for $30 \mathrm{~min}$. at $14,000 \times \mathrm{g}$ at $4^{\circ} \mathrm{C}$. Subsequently, supernatant was transferred to Amicon $10 \mathrm{~K}$ spin columns (Z677108-96EA, Sigma Aldrich). Spin columns were centrifuged at $14,000 \times \mathrm{g}$ for $20 \mathrm{~min}$. and filtrate was collected. Filtrate was used for lactate analysis using the Lactate Colorimetric Assay Kit II (K627, Biovision) according to the manufacturer's instructions. Briefly, filtrate was tested at three different dilutions; 5, 10 and 50 times diluted in "Lactate Assay Buffer" and 50- $\mu$ l sample or diluted sample was transferred to a 96-wells plate. Subsequently, 50- $\mu$ reaction mix composed of Lactate Substrate Mix (2- $\mu \mathrm{l})$, Lactate Enzyme Mix $(2-\mu \mathrm{l})$, and Lactate Assay Buffer $(46-\mu \mathrm{l})$, was added to each well and incubated for $30 \mathrm{~min}$ at room temperature. Optical density was measured at $450 \mathrm{~nm}$ and concentrations of extracellular lactate were calculated based on a lactate calibration curve supplied in the kit.

\section{Digesta nutrient composition analysis and digestibility calculation}

The digesta were ground using a lab jar mill (stainless) prior to the analysis, feed was analysed as whole pellets as described by Staessen et al. (2020). Collected digesta and feed were analysed gravimetrically for DM by drying at $103{ }^{\circ} \mathrm{C}$ for $4 \mathrm{~h}$ until constant weight. Following the DM determination, ash content was determined gravimetrically by incineration in a muffle furnace for $4 \mathrm{~h}$ at $550{ }^{\circ} \mathrm{C}$ (ISO 5984, 1978). Ashed samples were transferred to volumetric flasks and dissolved in concentrated sulphuric acid solution by autoclaving. Samples were 
subsequently diluted in water and filtered using a syringe filter (45 $\mu \mathrm{m}$ pores). Finally, yttrium $(Y)$, Phosphorous (P), calcium (Ca), magnesium ( $\mathrm{Mg}$ ), manganese $(\mathrm{Mn})$, iron (Fe) and zinc ( $\mathrm{Zn})$ were analysed using inductively coupled plasmamass spectrometry (ICP-OES) according to the standard NEN 15510 (2007). The total nitrogen content was measured in feed using the Kjeldahl-method (ISO 5983, 1997) and in digesta according to Dumas method (Ebeling, 1968), calculating CP as $\mathrm{N} \times 6.25$ (protein conversion factor).

The apparent digestibility coefficient (ADC) of mineral and $\mathrm{CP}$ was calculated as: $\operatorname{ADC}(\%)=100 \times\left[1-\left(Y_{i} \times\right.\right.$ amount nutrient in digesta $) /\left(Y_{f} \times\right.$ amount nutrient in feed)], where $Y_{i}(g / k g D M)$ is the concentration of yttrium in the feed and $Y_{f}$ ( $\mathrm{g} / \mathrm{kg} \mathrm{DM})$ is the concentration of yttrium in the digesta from the four sections along the GI-tract.

\section{DNA extraction and sequencing}

The distal gut samples were sent to BaseClear (Leiden, the Netherlands) for DNA extraction (ZYMO research, USA) and 16S rRNA sequencing, using in-house protocols. Paired-end sequence reads were generated using the Illumina MiSeq system. Initial quality assessment was based on data passing the Illumina Chastity filtering. The second quality assessment was based on the remaining reads using the FASTQC quality control tool version 0.11.5. Paired-end sequence reads were collapsed into so-called pseudoreads using sequence overlap with USEARCH version 9.2 (Edgar, 2010). Classification of these pseudoreads is performed based on the results of alignment with SNAP (version 1.0.23) against the RDP database for bacterial organisms.

\section{Microbial community analysis}

In total, 48 tilapia gut samples were sequenced of which 4 samples ( 1 from CONCON treatment and 3 from ENZ-CON) were removed from analysis due to the low sequencing depth. The sequencing data of the remaining 44 samples were rarified at the threshold of 27000 reads sequencing depth. Alpha-diversity was assessed using Shannon diversity index and observed richness (the number of Operational taxonomic units, OTUs) for each sample. Beta-diversity was assessed using the Bray-Curtis distance metric, and clustering analysis was performed by Principle Coordinate Analysis (PCoA), using the Primer software (Version 6).

Network construction was performed using CoNet (Faust and Raes, 2016) between the different treatment groups, to assess the microbial co-occurrence relationships between microbes, using the recommended parameters. For the network analysis, the following parameters were evaluated: A. Clustering coefficient, which is the ratio between existing and possible connections between a node's neighbours - it is measuring the degree to which several nodes in a network cluster together; $\mathrm{B}$. Network density, which is defined as the ratio of the number of total edges to the number of possible edges between all the nodes of the network; C. Network heterogeneity, which is an index that quantifies the diversity of connections 
between nodes in networks, even with different topologies - it ranges from 0 to 1 , with 1 referring to maximum heterogeneity, i.e. when each one of the nodes is connected to all the other nodes. This measure may indicate the stability and robustness of a network with respect to perturbations from various external factors. D. Positive to negative edges ratio, indicating co-presence versus mutual exclusion patterns.

\section{Statistical analysis}

The effect of enzyme and probiotic supplementation and their interaction on VFA and lactate concentration, minerals absorption and protein digestibility were tested by two-way ANOVA using general linear model in SPSS software (IBM, version 25). The significance of individual treatment was compared using Tukey HSD when the effect was significant $(P<0.05)$.

The alpha-diversity scores were compared among the four treatments by nonparametric t-test. The effects of enzymes and probiotics and their interactions on microbial composition were analysed by two-way PERMANOVA using Primer (Version 6). Moreover, correlations between the OTU matrix and fish body weight (BW), digestibility and VFA content in the distal gut were tested by distance based linear modeling (DistLM in Primer Version 6), and the results were visualized in the PCoA diagram. The similarity percentage among the four treatments and the contributions from each species were calculated by similarity percentage (SIMPER) analysis using PAST software (Version 4). For the microbial interactions, significance of the tested parameters was assessed by rarefying the table, using subsampling at 19000 reads sequence depth (70\% of the initial reads), and performing the analysis for each group using the Mann-Whitney test.

\section{Results}

\section{VFA and lactic acid content along the gastrointestinal tract}

The effects of the enzymatic cocktail and the probiotic mix supplementation on the VFA chyme content along the GI-tract are shown in Figure 1. The average VFA composition was similar at all four dietary treatments, indicating that both enzymes and probiotics supplementation did not affect the VFA composition. Acetic acid was the dominant VFA (86-88\%) along the GI-tract, followed by propionic acid (10-12\%) (Figure 1a), while low amounts of (iso) butyric acid and (iso) valeric acid (in total $1-3 \%$ ) were also detected (Table S1). The highest total VFA content was observed in the proximal gut. The stomach, middle and distal intestine had a similar total VFA content. Probiotics supplementation increased the total VFA content in the proximal gut $(P=0.005$; Figure $1 \mathrm{~b})$, but not in the other GI-tract segments $(P>0.1)$. Total chyme VFA content was unaffected by the enzymatic cocktail supplementation in all GI-tract segments $(P>0.1$; Figure $1 \mathrm{c})$. For none of the VFAs in any of the GI-tract segments, there was an interaction effect between enzymes and probiotics ( $P>0.1$; Table $S 1)$. 
Lactic acid was detected in fresh chyme in the Nile tilapia GI-tract, ranging from $0.08 \mathrm{mM}$ in the stomach to $0.43 \mathrm{mM}$ in the proximal gut (Figure 2; Table S2). Enzyme supplementation reduced the lactic acid concentration in the proximal, middle and distal gut $(P<0.05)$. In all GI-tract sections, lactic acid concentrations were unaffected by probiotics supplementation nor by the interaction effect between enzymes and probiotics supplementation $(P>0.1)$.

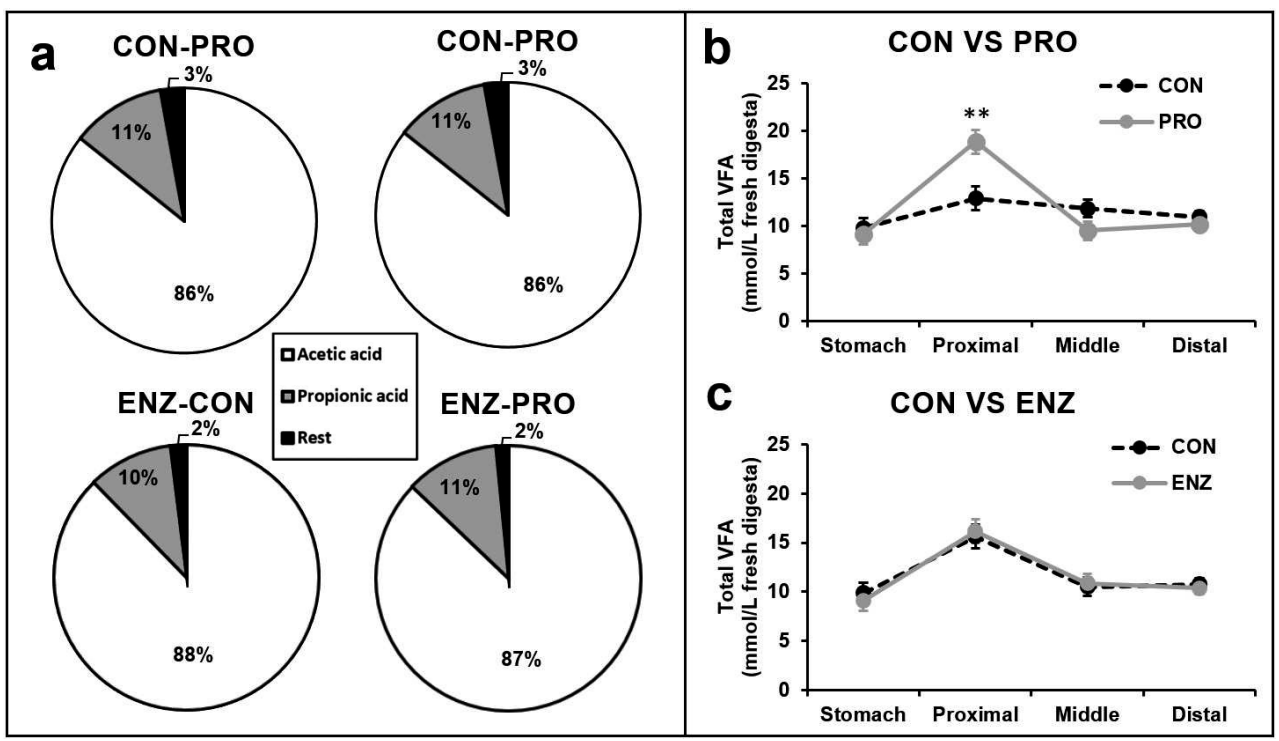

Figure 1 Volatile fatty acid (VFA) composition in fresh chyme along the gastrointestinal tract of Nile tilapia. CON-CON, no enzymes or probiotics added; CON-PRO, probiotics added; ENZ-CON, enzymes added; ENZ-PRO, enzymes and probiotics added. (a) The proportion of the two most abundant VFAs per dietary treatment averaged over the four segments of the gastrointestinal tract, the rest fraction consists of (iso) butyric and (iso) valeric acid. (b) The dietary effect of the probiotics mixture on the total VFA content in fresh chyme $(\mathrm{mmol} / \mathrm{L}$ ) along the gastrointestinal tract (CON vs. PRO, being respectively the diets without versus the diets with probiotic mix supplementation). (c) The dietary effect of the enzyme cocktail on the total VFA content in fresh chyme ( $\mathrm{mmol} / \mathrm{L})$ along the gastrointestinal tract (CON vs. ENZ, being respectively the diets without versus the diets with enzyme cocktail supplementation). Error bars indicate standard error of means; $* *$, $P<0.01$. 


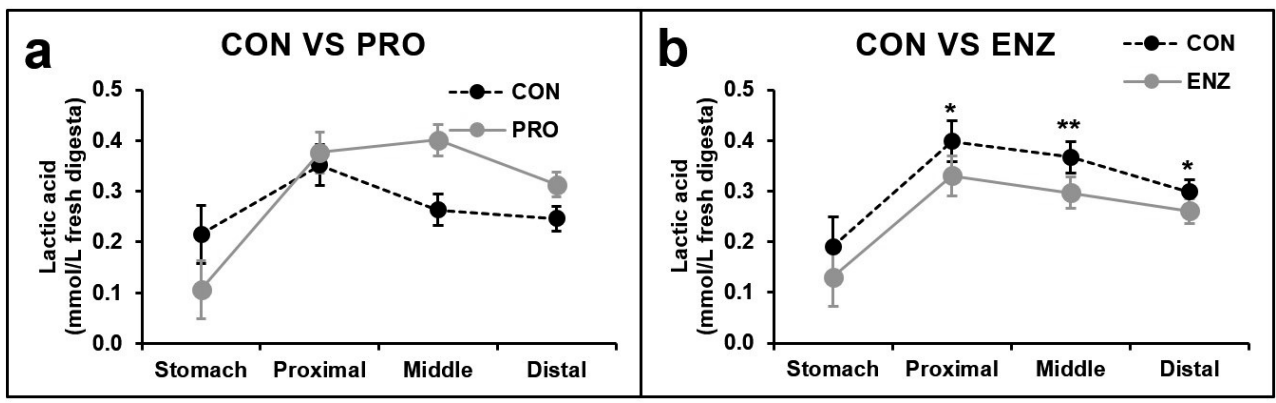

Figure 2 The lactic acid concentration in fresh chyme $(\mathrm{mmol} / \mathrm{L})$ along the gastrointestinal tract of Nile tilapia. (a) The effect of dietary probiotics mixture supplementation on the lactic acid concentration (CON vs. PRO, being respectively the diets without versus the diets with probiotic mix supplementation). (b) The effect of dietary enzyme cocktail supplementation on the lactic acid concentration (CON vs. ENZ, being respectively the diets without versus the diets with enzyme cocktail supplementation). Error bars indicate standard error of the mean; $*, P<0.05 ; * *, P<0.01$.

\section{Minerals absorption and crude protein digestibility along the gastrointestinal tract}

The main effects of probiotics and enzymes supplementation on kinetics of apparent digestibility coefficient $(A D C)$ of $C P$, phosphorous, calcium and magnesium of Nile tilapia are shown in Figure 3. The mean ADC data for each of the four dietary treatments are shown in Table S3, and the results from the distal gut are summarized in Table 1 . Average over all dietary treatments, large differences were present between ADC values in the different parts of the GI-tract. For most nutrients, digestion started in the stomach indicated by the small positive ADC values, but the largest part of digestion took place in the mid intestine. For $\mathrm{CP}$ as well as most minerals, the ADC in the proximal intestine was lower than the $A D C$ in the stomach. For $C P, C a$ and $M g$ even negative $A D C$ values were observed in the proximal intestine, most likely related to minerals and endogenous enzyme secretion in the proximal intestine (Figure 3). Enzymes supplementation increased the ADC of $\mathrm{CP}$ in the proximal and middle intestine $(P<0.05)$. This enzyme supplementation effect was absent in the distal intestine but was present again for fecal ADC of CP $(P<0.001$; Figure $3 b)$. ADC of $P$ was similar in the stomach, but enhanced by enzyme supplementation from the proximal intestine onward $(P<0.01$; Figure 3d). The difference between diets with and without enzyme supplementation remained relatively constant from the proximal intestine up till faeces. Parallel to the absorption of $\mathrm{P}$, enzymes supplementation improved $\mathrm{Ca}$ absorption but this effect appeared more distal in the GI-tract (Figure 3f). The absorption of $\mathrm{Mg}$ was numerically higher in diets supplemented with enzymes but this effect was only significant for faecal Mg ADC (Figure 3g). 


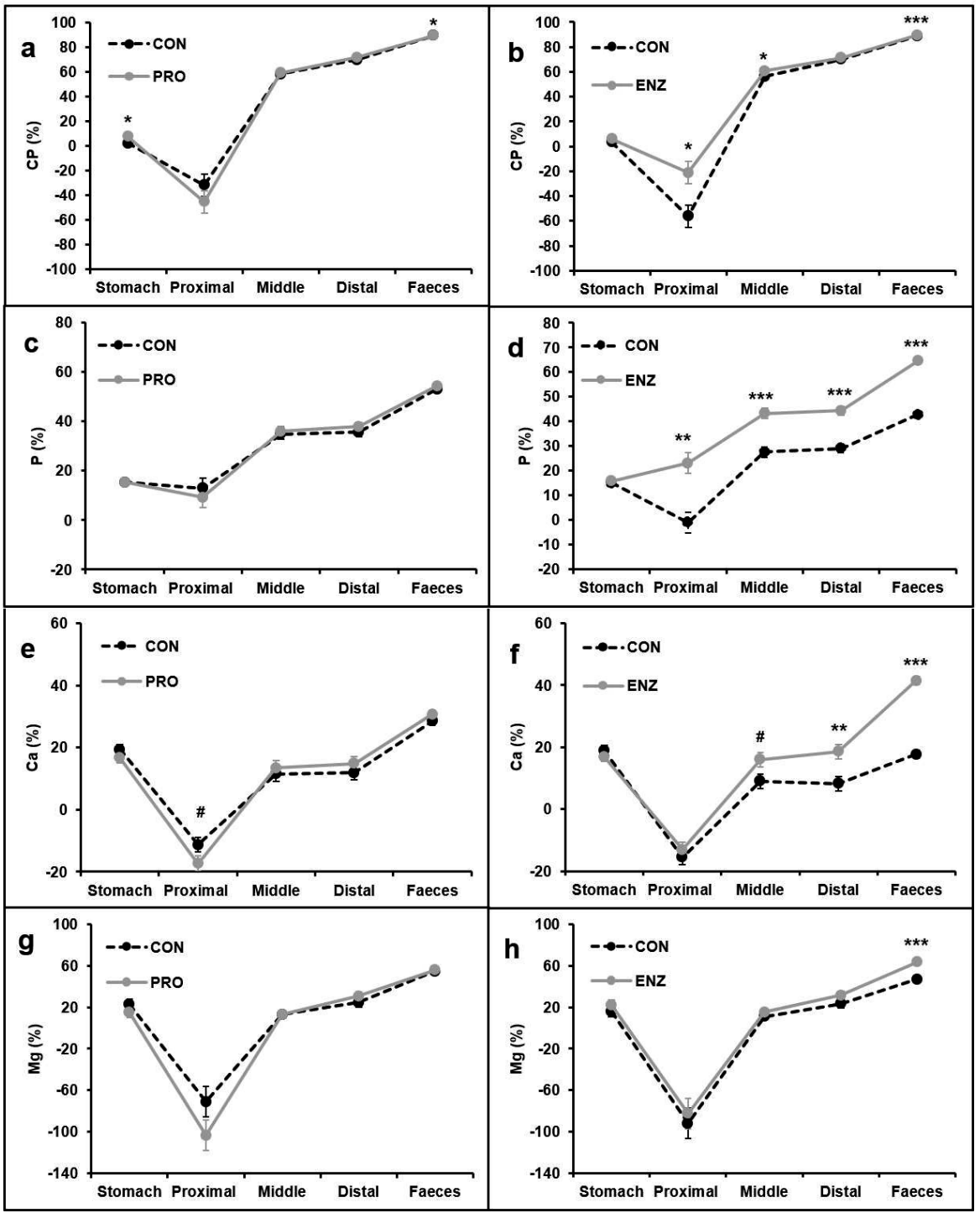

Figure 3 The main effect of probiotic mix and enzymes cocktail supplementation on the kinetics of apparent digestibility coefficient (ADC) in Nile tilapia along the gastrointestinal tract. The ADC of: crude protein (CP) (panel $a$ and $b$ ); phosphorous ( $P$ ) (panel $c$ and $d$ ); calcium (Ca) (panel e and f); and magnesium (Mg) (panel $g$ and $h$ ). In panel a, c, e and $g$ (left side) the main effect of dietary probiotics mixture supplementation on ADC values is shown (CON vs. PRO, being respectively the diets without versus the diets with probiotic mix supplementation) and in panel $b, d, f$ and $h$ (right side) the main effect of dietary enzyme cocktail supplementation on ADC values (CON vs. ENZ, being respectively the diets without versus the diets with enzyme cocktail supplementation). The ADC in the stomach, proximal, middle and distal were determined on collected digesta in current study. The fecal ADCs were determined on faeces collected by settling columns during the growth trail (Chapter 7). Error bars indicate standard error of the mean. 
In Table 1, the ADC of DM, CP and all measured minerals up till the distal intestine is given. At all dietary treatments, $\mathrm{ADCs}$ of $\mathrm{P}, \mathrm{Ca}, \mathrm{Mg}$ and $\mathrm{Cu}$ were larger than zero, while ADCs of $\mathrm{Fe}, \mathrm{Zn}$ and $\mathrm{Mn}$ were negative at all treatments. ADC values including the distal intestine were enhanced by dietary enzyme supplementation for the macro-minerals, $\mathrm{Ca}$ and $\mathrm{P}(P<0.01)$. In contrast, these ADCs for the microminerals, Fe and $\mathrm{Zn}$, were lower (i.e., more negative; $P<0.01$; Table 1 ). Probiotics supplementation only affected the distal ADC of $\mathrm{Mn}(P<0.001$; Table 1$)$, being more negative at the diets with probiotic supplementation. None of the distal ADC values were influenced the interaction effect between probiotics and enzyme supplementation $(P>0.1)$.

Table 1 The apparent digestibility coefficient (ADC, \%) in the distal gut of Nile tilapia, values for stomach, proximal and middle are given in supplemental table S3.

\begin{tabular}{|c|c|c|c|c|c|c|c|c|}
\hline \multirow[t]{2}{*}{ Treatment } & \multicolumn{2}{|c|}{ CON } & \multicolumn{2}{|c|}{ ENZ } & \multirow[b]{2}{*}{ SEM } & \multicolumn{3}{|c|}{$P$ values } \\
\hline & CON & PRO & CON & PRO & & ENZ & PRO & ENZ*PRO \\
\hline \multicolumn{9}{|l|}{$A D C(\%)$} \\
\hline Dry matter & 45.7 & 47.0 & 46.5 & 49.2 & 1.6 & ns & ns & ns \\
\hline Crude protein & 69.7 & 70.4 & 69.7 & 72.7 & 1.5 & ns & ns & ns \\
\hline Ash & -6.8 & -6.4 & 3.6 & 6.5 & 2.1 & * & ns & ns \\
\hline Phosphorous & 29.2 & 28.9 & 42.0 & 46.8 & 1.2 & $* * *$ & ns & ns \\
\hline Calcium & 7.1 & 9.3 & 16.8 & 20.4 & 1.6 & $* *$ & ns & ns \\
\hline Copper & 27.7 & 30.2 & 15.8 & 17.6 & 4.0 & ns & ns & ns \\
\hline Magnesium & 21.9 & 25.2 & 27.0 & 35.9 & 2.8 & ns & ns & ns \\
\hline Iron & -34.1 & -26.2 & -36.8 & -59.4 & 1.3 & $*$ & ns & \# \\
\hline Manganese & -6.0 & -22.1 & -9.2 & -24.8 & 4.0 & ns & $* * *$ & ns \\
\hline Zinc & -10.3 & -7.0 & -13.5 & -19.2 & 1.6 & * & ns & ns \\
\hline
\end{tabular}

Notes. CON, no supplementation; ENZ, enzyme (effect) supplementation; PRO, probiotic (effect) supplementation; ENZ*PRO, interaction effect; SEM, standard error of the mean; ns, not significant; $\#, P<0.1 ; *, P<0.05, * * *, P<0.001$.

\section{Microbial community structure and composition}

There was no significant difference between the treatments in the alpha-diversity

- Shannon index and richness - and beta-diversity, as indicated by the PCOA and PERMANOVA analysis (Figure 4). However, when the bacterial community (i.e. OTU table) in distal gut was further correlated with the final fish body weight (BW), VFA content and nutrient $A D C$ in the distal gut, a weak but significant positive correlation was found with the propionic acid content (Figure $4 c ; r^{2}=0.136$; $P=0.045)$. 
a

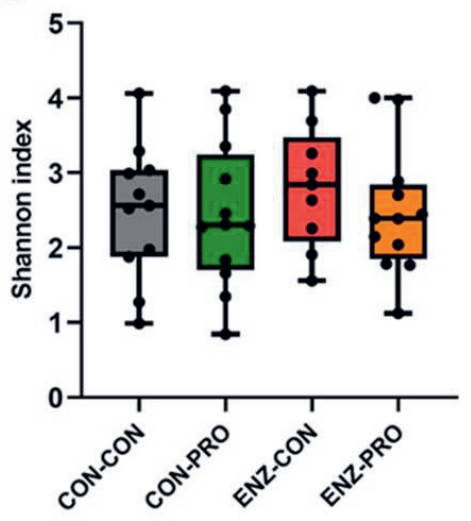

b

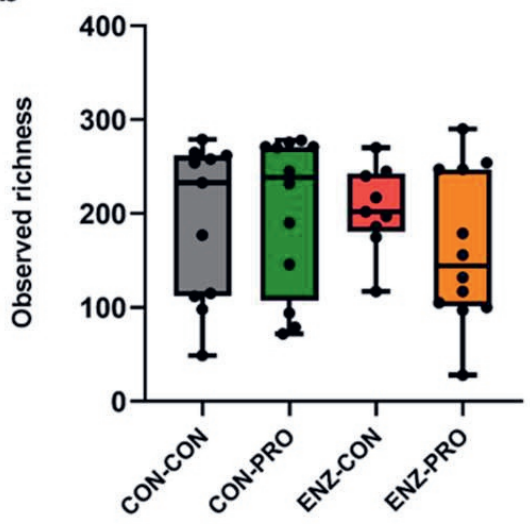

C

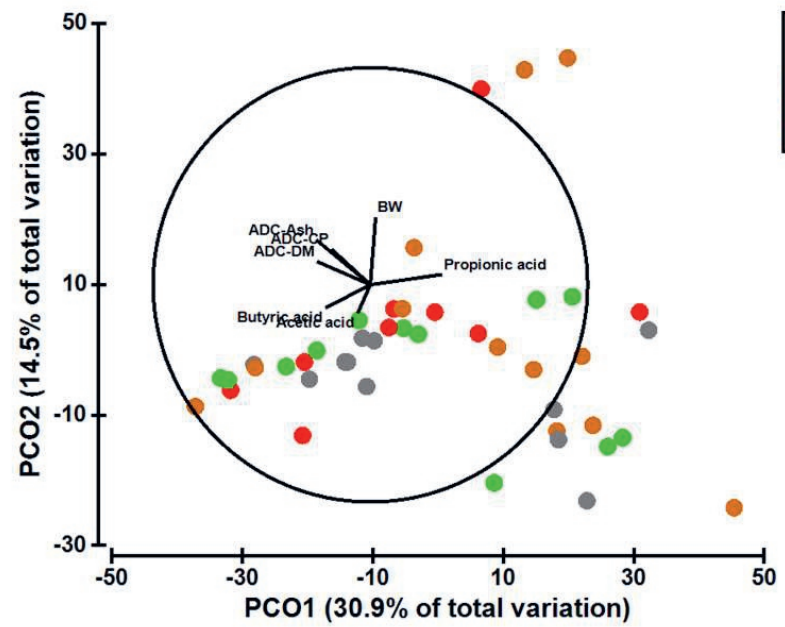

Figure 4 Microbial diversity in the distal gut of Nile tilapia. CON-CON, no enzymes or probiotics added; CON-PRO, probiotics added; ENZ-CON, enzymes added; ENZ-PRO, enzymes and probiotics added. (a) Shannon index, (b) observed richness, (c) PCoA based on Bray-Curtis distance and the correlation between microbial community structure and growth-related parameters. BW, body weight, ADC, apparent digestibility coefficient. Acetic acid, butyric acid and propionic acid are the concentrations from the distal gut. 
At the phylum level composition (Figure 5a), Fusobacteria was the most abundant group (36.2\%), followed by Bacteroidetes (15.5\%) and Firmicutes (13.5\%). Overall, 5 OTUs were found to contribute to $50 \%$ of the total dissimilarity between the treatments in microbial composition, as indicated by SIMPER analysis (Figure 5b). Cetobacterium somerae was the most dominant species (36.1\% of the total abundance) contributing to $23.0 \%$ of the dissimilarity among the dietary groups. The others four species, including Brevinema andersonii, Bacteroides stercoris, Romboutsia sedimentorum and Paludibacter propionicigenes, were also dominant species ( $27 \%$ of the total abundance), accounting for $28.9 \%$ of the dissimilarity among the dietary treatments. Enzyme supplementation increased the relative abundance of $B$. stercoris, $R$. sedimentorum and $P$. propionicigenes while for $B$. andersonii, a positive effect of probiotics was observed. Enzyme and probiotic supplementation seem to have an overall positive effect on the lactic acid bacteria abundance (Lactobacillales order; Supplementary Figure S1) as well as in the abundance of OTUs belonging to the Bacillus genus (Supplementary Figure S2).

\section{Microbial interaction network analysis}

A co-occurrence network analysis was performed to understand how microbial interactions may be affected by enzyme and probiotic dietary supplementation. Overall, after permutations and multiple correction tests, the co-occurrence network was mainly occupied by OTUs belonging to Proteobacteria, Bacteroidetes, Actinobacteria and Firmicutes phyla (Figure 6), which are amongst the most abundant ones.

Regarding the network characteristics (Table 2), supplementation of probiotics or enzymes increased the total number of both nodes and edges in the network. A significant increase in the clustering coefficient and density of the network was observed by probiotics supplementation. Enzyme supplementation in the diets also increased the density of the network. The ratio of positive to negative edges was significantly reduced with enzyme and probiotic supplementation. These results indicate that mainly probiotics, and to some extent also enzyme supplementation, enhanced species-species (co-occurrence patterns) interactions, as well as the type of those interactions. 


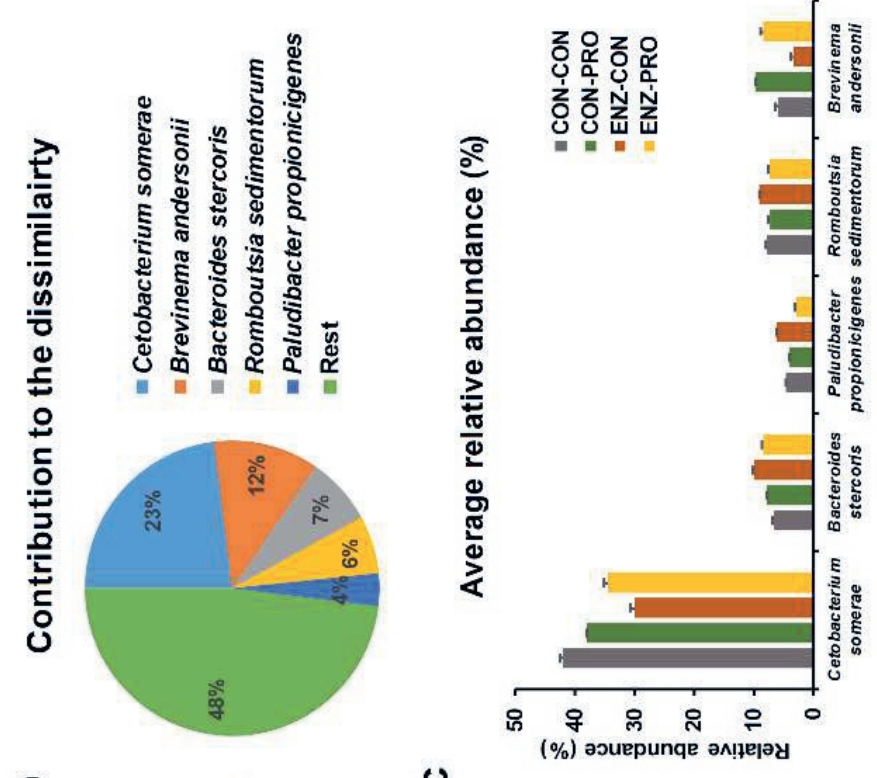

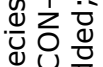

के त्र

เก \& บ

응 엄

응 की

닌 은

을 웅

क्ष

त्र

등 슨

$+5$

둥 -

동

กั

元

㻤

ह $\frac{0}{0}$

(1) 뭉

돈

ᄂ

ช ठ ते

㽞宁

든

긍ㅇํㅇ

(0)

$\stackrel{2}{\geq}$

范薄

닌 $\frac{0}{0}$

$\stackrel{0}{0}$

पु

징ㄷํ윰

을

年

음 웅

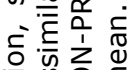

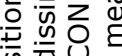

次

등 은 눙

웡

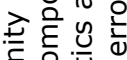

ร원음으

ह

응 는

就出

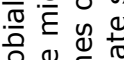

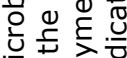

之

in

ข 킁응 용

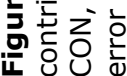

ס 


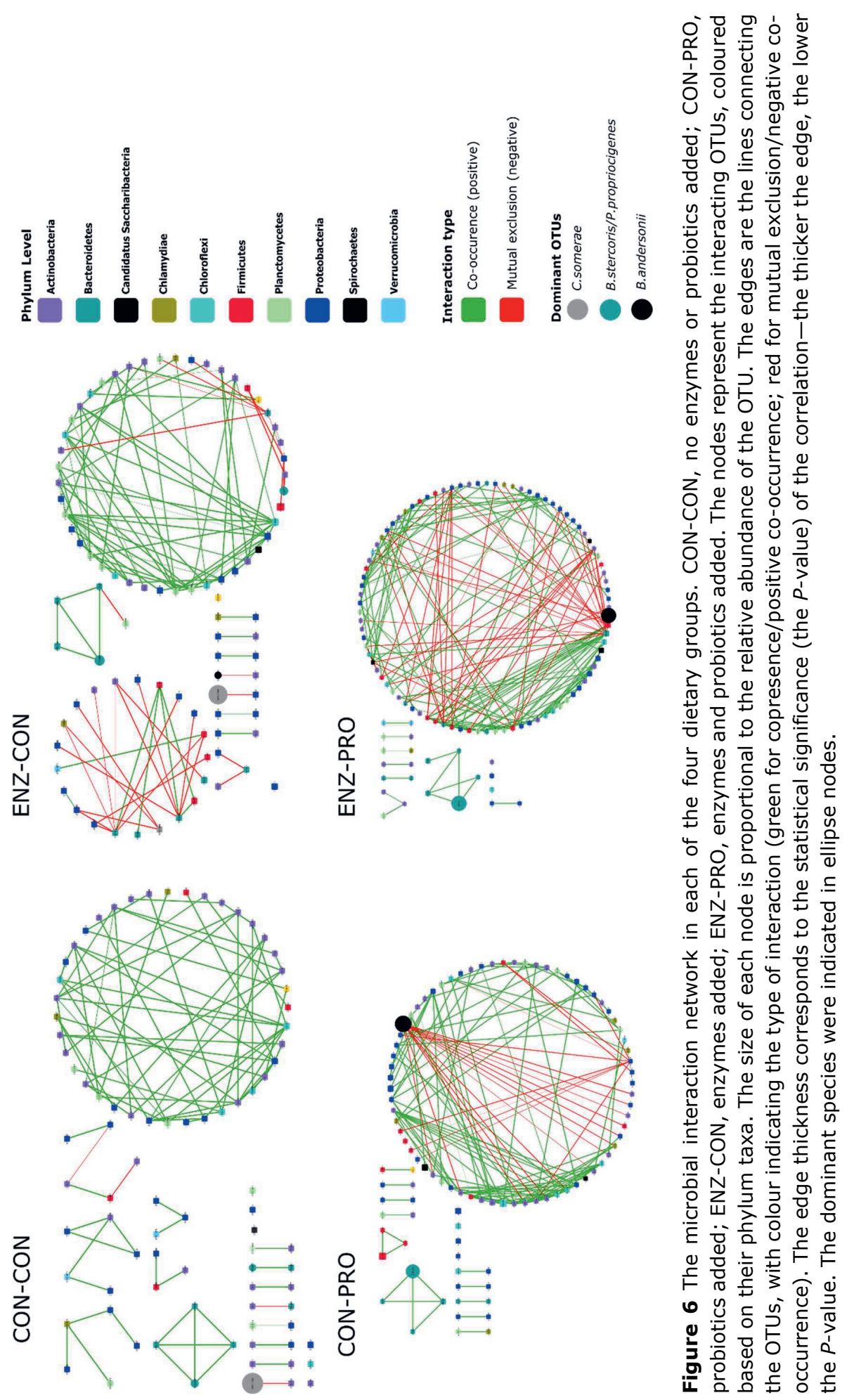


Table 2 the characteristics of the networks from each dietary treatment.

\begin{tabular}{lcccccc}
\hline Treatment & \multicolumn{2}{c}{ CON } & & \multicolumn{3}{c}{ ENZ } \\
\cline { 2 - 3 } \cline { 5 - 6 } & CON & PRO & & CON & PRO \\
\hline Number of nodes & 86 & 98 & & 90 & 112 \\
Number of edges & 94 & 146 & & 118 & 186 \\
Number of positive edges & 90 & 122 & & 86 & 137 \\
Number of negative edges & 4 & 24 & & 32 & 49 \\
Positive/Negative ratio & 23 & $5^{*}$ & & $3^{*}$ & $3^{*}$ \\
Clustering coefficient & 0.19 & $0.22^{*}$ & & 0.18 & $0.24^{*}$ \\
Density & 0.025 & $0.031^{*}$ & & $0.029^{*}$ & $0.03^{*}$ \\
Heterogeneity & 0.77 & 0.98 & & 0.86 & 0.96 \\
\hline
\end{tabular}

Notes. CON, no supplementation; ENZ, enzyme supplementation; PRO, probiotic supplementation *Stars indicate significant differences from the CON-CON treatment at $P<0.05$, after performing the permutations analysis ( $70 \%$ of the initial data).

\section{Discussion}

\section{Volatile fatty acids and lactate content along the gastrointestinal tract}

The digestion of NSP in tilapia is expected to be through anaerobic microbial glycolysis (fermentation), producing VFAs (Metzler-Zebeli et al., 2010; Piazzon et al., 2017; Maas et al., 2020a). In the present study, we expected that the supplementation of cocktail containing xylanase (NSP-degrading enzyme) and phytase would improve the NSP digestibility, as previously shown in the Nile tilapia (Maas et al., 2020b). Such supplementation would stimulate the breakdown of the polysaccharides into readily available oligomers and monomers for fermentation, increasing the production of beneficial VFA and improving the nutrient utilisation. The enzyme supplementation in the present study did increase the NSP digestibility by approximately $29 \%$ (shown in chapter 7 ). However, this increase did not go hand in hand with an increase in VFA levels, which remained unaffected by the enzyme supplementation (Figure 1c). On the contrary, the supplementation of probiotics increased the level of acetic acid as well as the total VFA in the proximal part of the GI-tract (Figure 1b). Similarly, a strain affiliated to $B$. amyloliquefaciens was reported to dramatically increase the concentration of acetate in the intestine of Nile tilapia, which could be explained by the enrichment of the short-chain fatty acid-producing bacteria (Xu et al., 2020). Unfortunately, the microbial community in the proximal gut, where major changes were observed in our study, was not sampled and analysed. This should be considered for future research.

Overall, the total concentration of VFA along the GI-tract remained rather constant, with averages between $9.5-10.7 \mathrm{mmol} / \mathrm{L}$ fresh digesta. Generally, it is shown that the concentration of VFA increases towards the distal intestine, including two studies on Nile tilapia (Amirkolaie et al., 2006; Leenhouwers et al., 2007a). This coincides with increased microbial activity and anaerobic conditions 
in the distal gut (Maas et al., 2020a; Mountfort et al., 2002). The average concentration of total VFA found in the distal gut within the present study was 10.6 $\mathrm{mmol} / \mathrm{L}$ fresh digesta, which is close to the average reported across fish species (with predominately herbivorous feeding habits), but considerably lower compared to the studies on Nile tilapia where on average 16.9 and $17.3 \mathrm{mmol}$ VFA/L fresh digesta was found (Amirkolaie et al., 2006; Leenhouwers et al., 2007a). It could be speculated that the relative high levels of VFA found in the proximal gut of the intestine could have reduced the available substrate for fermentation in the distal part, thereby reducing the potential of VFA production. This is supported by the observation that the cumulative total VFA content of the different sections of the GI-tract (stomach + proximal + middle + distal) in this study, is highly comparable to the total VFA content in the GI-tract of Nile tilapia (Amirkolaie et al., 2006; Leenhouwers et al., 2007a). However, what caused this relative high levels of VFA observed in the proximal gut in this study remains unclear.

While several studies have investigated lactate in serum or under in vitro conditions in fish (Petit et al., 2019b; Wentzel et al., 2020; Zhang et al., 2018), the present study is the first to measure the presence of lactate in the intestinal content of fish in vivo. Compared to the concentrations of the other VFAs, the observed level of lactate was very low. Nevertheless there was a clear trend visible in the concentrations over the different compartments of the GI-tract, i.e. low lactate concentrations in the stomach, relatively high in the proximal compartment and gradually decreasing towards the distal intestinal compartment. Interestingly, significant differences were observed among the dietary treatments. Supplementation with both xylanase and phytase resulted in lower lactate concentrations in each of the intestinal segments, except for the stomach. Possibly, the enzymatic supplementation has sped up the initial fermentation of oligosaccharides into acetic acid and lactic acid, resulting in earlier secondary fermentation of lactic acid into other VFAs, such as propionic acid and butyric acid (Bedford and Cowieson, 2012). Coinciding with the decrease in lactic acid, an increase in the presence of lactic acid bacteria was measured. While these bacteria predominantly produce lactic acid, it has been shown that lactic acid bacilli can also catabolize lactic acid into other compounds such as acetate (Liu, 2003). Further supported by an increase in species - species interaction that was observed following enzymatic supplementation.

In line with what is observed in other studies on fish, acetate (86-88\%) is the dominant VFA found in the present study followed by propionate (10-12\%) and the rest fraction (1-3\%), of which butyrate forms the bulk (Maas et al., 2020a). In other monogastric animals, the order of prevalence of the different types of VFA is the same, although with a more proportional distribution; 60-75 acetate, $15-25 \%$ propionate and $10-15 \%$ butyrate (Bugaut, 1987). In monogastric animals, VFA produced can be rapidly absorbed in the colonic lumen, with $95-99 \%$ of the VFA production being absorbed before reaching the rectum (Scheppach, 1994; Von Engelhardt et al., 1989). From studies in poultry and pigs it is known that the route of uptake differs between types of VFA; acetate and propionate enter the blood 
passively, whereas butyrate is primarily used as direct source of energy by the colonocytes (Montagne et al., 2003; Von Engelhardt et al., 1989; Williams et al., 2001). Likewise, we expect that in fish most of the VFA produced is rapidly absorbed, whereby it remains unclear whether the rate of uptake is different between acetate, propionate and butyrate. Therefore it is difficult to quantify the amount and composition of VFA produced in vivo based on measuring concentration in chyme, and such data should be interpreted with care.

\section{Nutrient digestibility and digestion kinetics along the gastrointestinal tract}

Examining the digestibility coefficients along the GI-tract broadens insight in the digestion kinetics in fish, considering such studies in fish are rare. The digestion kinetics can enable us to pinpoint the locations in the GI-tract where probiotics and enzymes are active, or where minerals become available. One of the main goals of the present study was to increase $P$ availability from dietary phytate by including exogenous enzymes in the diet, in order to reduce the dependency of finite $P$ sources. In line with many studies using phytase, the enzyme supplementation improved the overall $\mathrm{P}$ availability (Kumar et al., 2012; Liebert and Portz, 2005; Maas et al., 2020b; Oliva-Teles et al., 1998) throughout the GItract, starting from the proximal gut. Hereby the difference in $P$ availability between enzyme supplementation (average ENZ-CON and ENZ-PRO) and no supplementation (average CON-CON and CON-PRO) in the proximal and complete gut was comparable with $20 \%$ and $23 \%$, respectively. This indicates that the enzymes were active in the stomach and potentially in the proximal gut as enzymes supplementation did not further enhance the $\mathrm{P}$ availability. The activity of commercial phytase products is generally in a $\mathrm{pH}$ range of 2.5-5.5 (Dersjant-Li et al., 2015; Kumar et al., 2012). The low pH in the stomach helps to quickly break down phytate before it chelates with $\mathrm{Ca}^{+2}$ and other minerals. In tilapia, the $\mathrm{pH}$ in the stomach ranges from 1 to 4.5 (Leenhouwers et al., 2007a; Saravanan et al., 2013), depending on the time post prandial feeding and the dietary composition (Saravanan et al., 2013). From the proximal to the distal intestine, the $\mathrm{pH}$ is rather constant ranging between 6.4 to 7 (Leenhouwers et al., 2007a). Such an increase in the $\mathrm{pH}$ after the stomach deactivates phytase activity in the GI-tract, therefore we expect phytase mainly to be active in the stomach. Although $\mathrm{P}$ is not absorbed in the stomach, in the current study we observed $P$ availability between $13-18 \%$ (unaffected by treatment), while $P$ availability is almost $0 \%$ in the proximal intestine when no enzyme was supplemented. It is known that low $\mathrm{pH}$ increases the solubility of several minerals (NRC, 2011); such increased solubility can lead to a faster evacuation (liquid fraction) from the stomach to the proximal intestine, thus overestimating the availability of these minerals (including $P$, especially the monocalcium phosphate).

In this study, probiotics supplementation showed no effect on the digestion kinetics along the GI-tract, except a negative effect on Mn (Table 1). Certain strains that belong to $B$. amyloliquefaciens can synthesize many enzymes including amylase, cellulase, and xylanase, and therefore could potentially increase nutrient 
digestibility (Deb et al., 2013; Farhat-Khemakhem et al., 2018; Lee et al., 2008). However, no beneficial effect of probiotics supplementation on nutrient digestion kinetics was observed in this study, except for improved fecal fat digestibility (Chapter 7).

When looking at the trend of availability of the other minerals ( $\mathrm{Ca}, \mathrm{Cu}, \mathrm{Mg}, \mathrm{Fe}, \mathrm{Mn}$, $\mathrm{Zn})$ and protein digestibility along the GI-tract, a clear drop in availability/digestibility is observed in the proximal gut causing negative ADC values. The drop in ADC is most likely the consequence of the endogenous secretion of minerals to maintain homeostasis and the secretion of for instance enzymes like chymotrypsin's (from the pancreas) and components containing $\mathrm{N}$ like bile acids, which in fish is generally conjugated with taurine (Case, 1978; Hagey et al., 2010). After the proximal intestine, the drop in availability/digestibility was quickly compensated.

In the current study there was a trend for a lowered Ca availability only in the proximal intestine with dietary probiotic supplementation (Figure 2e). This might relate to increased VFA concentration with probiotic supplementation (Figure 1b). Bicarbonate secretion $\left(\mathrm{HCO}^{3-}\right)$ in the proximal intestine is required to quickly neutralise the low $\mathrm{pH}$ of the stomach, which enters the proximal intestine (Cooper et al., 2010; NRC, 2011). The higher VFA levels in the proximate intestine associated with probiotic supplementation might lead to an increased secretion of bicarbonate to increase the $\mathrm{pH}$ to normal levels. The secretion of bicarbonate is under control of multiple cellular signalling pathways in which $\mathrm{Ca}^{2+}$ plays an major role (Jung and Lee, 2014). Therefore, higher secretion of bicarbonate is expected to be linked with a higher influx of $\mathrm{Ca}$ into the proximal intestine, which might be the reason for the observed trend for a reduced $\mathrm{Ca}$ availability in the proximal intestine in fish fed probiotics supplemented diets. Ca plays an important role in the digestion of lipids. Ca is known to precipitate accumulated free fatty acids, thereby $\mathrm{Ca}$ can enhance the accessibility of lipase to the emulsified lipids, leading to an increased lipase (Fave et al., 2004; Hu et al., 2010). In this study, probiotics resulted in a higher fat digestibility (data shown in chapter 7 ), which may be linked to this theorem.

The enzyme supplementation enhanced the ash, $\mathrm{Fe}$, and $\mathrm{Zn}$ availability in the distal, as well as protein digestibility (data shown in chapter 7). Phytate is known to reduce the availability of minerals by chelating with cations such as $\mathrm{Ca}^{2+} \mathrm{Mg}^{2+}$ $\mathrm{Zn}^{2+}$ and $\mathrm{Fe}^{3+}$, forming complexes. Likewise phytate can form complexes with amino-acids complexes, which can lead to reduced protein digestibility (PimentelRodrigues et al., 2007; Kumar et al., 2012). Therefore, reducing the amount of phytate with phytase is expected to partly counteract its negative effects, which may result in improved mineral availability and protein digestibility as shown in the present study. 


\section{Microbial community composition and co-occurrence networks}

The beneficial effects of $B$. amyloliquefaciens as a probiotic on Nile tilapia have been evaluated in several studies aiming on fish growth performance and immune response (Ridha and Azad, 2012; Selim and Reda, 2015 Al-Deriny et al., 2020; Kuebutornye et al., 2020). However, the effect of the probiotic $B$. amyloliquefaciens on the gut microbiota composition still remains unclear. Moreover, several studies have evaluated the addition of exogenous enzymes on the gut microbiota of Nile tilapia, turbot and grass carp, showing alterations in the microbial communities, and in some cases even an increase in microbial diversity and richness (Zhou et al., 2013; Adeoye et al., 2016a; Diógenes et al., 2018). The combination of enzymes and probiotics in the diets can have complimentary effects by increasing availability of substrates for the probiotic and promoting the abundance of beneficial bacteria, while increasing fibre degradation. In this study, we evaluated the effects of enzyme and probiotic supplementation in the distal gut microbial communities. No significant impact was observed on the richness and diversity between the dietary treatments, potentially due to the large variations among the individuals (Figure 2). In a study with Nile tilapia, exogenous enzymes (containing phytase, protease and xylanase) and probiotics (containing Bacillus subtilis, Bacillus licheniformis and Bacillus pumilus) supplementation mildly altered the diversity of the microbial community in the fecal matter (Adeoye et al., 2016b). Interestingly, the authors showed a small increase, although not significant, in Bacillus species abundance in the gut with probiotic supplementation. In our study, we observed a higher abundance of Bacillus species in the groups fed with enzyme and probiotic supplementation (Supplementary Figure S2), showing a potential positive impact on GI-tract health. Indeed, our VFA and lactic acid analysis indicated a higher microbial activity when probiotics were added. Although this was observed only in the proximal intestine and cannot be directly connected to microbial composition changes, such results indicate potential benefits of probiotic and enzyme supplementation for gut health.

In this study, we evaluated the effects of enzyme and probiotic supplementation in the distal gut microbial communities. No significant impact was observed on the richness and diversity between the dietary treatments, potentially due to the large variations among the individuals (Figure 2). Another study with Nile tilapia indicated that exogenous enzymes (containing phytase, protease and xylanase) and probiotics (containing Bacillus subtilis, Bacillus licheniformis and Bacillus pumilus) supplementation mildly altered the diversity of the microbial community in the fecal matter; however in this study, the microbial communities in the middle intestine were evaluated (Adeoye et al., 2016b). From our VFA analysis, a higher microbial activity was hypothesised to occur in the proximal intestine, where a higher VFA production was observed. However, we did not sample this compartment for microbial analysis, therefore, we cannot make a direct analogy with the microbial composition. 
The microbial food webs in the GI-tract are built largely from the nutrients their host consumes, which act as one of the most important factors in shaping composition and metabolism of the intestinal microbiome (Nayak, 2010). Our analysis showed that the dominant microbial species detected in the distal gut of Nile tilapia were related with protein and carbohydrates metabolism, which could be explained by the high levels of plant materials, including NSP, in the diet. $C$. somerae is an anaerobic microbe which is capable of producing vitamin B12 in fish intestine (Sugita et al., 1991) and is related to fermentative metabolism of peptides and amino acids (Finegold et al., 2003). This species has been also reported to produce antimicrobial peptides, that allow it to eliminate other microbes and to occupy most niches in the fish GI-tract (Sugita et al., 1996). Our study confirms $C$. somerae to be one of the dominant microbial species in Nile tilapia GI-tract, potentially due to highly available substrate in the diet and high competitive potential of this taxon. Brevinema andersonii was found to be the second dominant species in the Nile tilapia GI-tract in our study. This species has not been reported before as a dominant species in the Nile tilapia GI-tract; however, it was previously reported as a dominant species in the GI-tract of Atlantic salmon (Salmo salar), when fed with diets supplied with alginate oligosaccharide. This species was reported to carry genes that are necessary for butyrate production (Gupta et al., 2019) and are related to the immune responses of Atlantic salmon ( $\mathrm{Li}$ et al., 2020). Interestingly, a trend for an increase in abundance of $B$.andersonii was found with the addition of probiotics in our study (although not significant; Figure 5c); such a trend for increase was also observed in the butyric acid content in the proximal gut in fish fed with probiotics (Table S1), implying that probiotics may stimulate the abundance of this species as well as the butyric acid production in the gut. Romboutsia sedimentorum and Bacteroides stercoris are both obligatory anaerobic microbes (Wang et al., 2015), with a broad range of metabolic capabilities with respect to carbohydrate utilisation and anaerobic respiration (Gerritsen et al., 2019). In our study, both microbial species increased in abundance with enzyme supplementation (Figure 5c), potentially explained by an increased in carbohydrate substrate. Paludibacter propionicigenes is a strictly anaerobic, propionate-producing microbe (Ueki et al., 2006). From our study, propionic acid was positively correlate with microbial composition and increased in the distal gut with enzyme supplementation; that could be explained by the increase in the abundance of this species.

Probiotics supplementation (CON-PRO and ENZ-PRO) increased the species interactions in the distal gut microbiota when compared with the control treatment (CON-CON). A previous study showed that probiotics enhanced the species-species interaction network in the hindgut of sea cucumber, by increasing the network complexity (clustering co-efficiency and density), which was hypothesised to benefit the intestinal microbiota homeostasis (Yang et al., 2017). Besides, we also found that probiotics supplementation enhanced the growth of tilapia with a smaller fraction of dietary energy going to maintenance (chapter 7). A stable intestinal microbiota might be beneficial to the fish with high NSP diet inferences, thus reducing the energy requirement for maintenance. Interestingly, we found 
that the ratio of positive to negative interactions (co-occurrence patterns) decreased with the addition of the enzymes and probiotics. That could be explained by the breaking down of more complex polysaccharides in the diets, which can serve as a food source for specific microbes (Moraïs and Mizrahi, 2019). More complex carbohydrates as substrates create different trophic levels in the GI-tract (different microbes are able to utilize different substrates) thus can potentially support higher diversity (Nishida and Ochman, 2018). By partly digesting some of those sources, such trophic levels might have been disrupted, creating an environment with high availability and less complexity of substrates in which more microbes have to compete for the same substrates. In the case of the probiotic presence, a higher production of VFAs by the microbial communities may lead to lower $\mathrm{pH}$, thus negatively affecting certain taxa that are less tolerant to such conditions. This agrees with the higher abundance of lactic acid bacteria (Lactobacillales) with probiotic and enzyme supplementation (Supplementary Figure S1), leading to higher lactic acid production and a lower $\mathrm{pH}$. Looking at the network ENZ-PRO, the negative interactions mainly originated from species of the order Clostridiales, which are known to consist of many fermentative species, producing VFAs (Clements et al., 2014). In the network CON-PRO, the negative interactions mainly originated from $B$. andersonii, which is a butyrate-producing bacteria (Gupta et al., 2019), and was found to increase when probiotics were added, thus potentially explaining the negative impact on other microbial species. Therefore, dietary supplementation of probiotics and enzymes can modify the structure of the microbial communities in the Nile tilapia GI-tract by altering carbohydrate substrates and VFA production, while enhancing microbial interactions and thus increasing microbiome stability and GI-tract health.

\section{Conclusion}

To summarize, probiotics supplementation enhanced the total VFA concentration in the proximal GI-tract. Apart from this increase, the total VFA concentration along the GI-tract remained rather stable. The contribution of acetic acid to the total concentration of VFA was high with $86-88 \%$, whereby the composition of VFA was not influenced by the dietary treatment, nor did it alter along the GI-tract. The results on the digestion kinetics suggest that the enzymes were mainly active in the first part of the GI-tract, with a strong effect on the P availability, which is likely to be linked with a low $\mathrm{pH}$. In line with a higher $\mathrm{P}$ availability (and thus expected lower levels of phytate), the availability of calcium, iron, zinc and the apparent digestibility coefficient of the ash fraction was higher with enzyme supplementation. Enzymes and probiotics supplementation did not affect gut microbial composition in the distal gut. The microbial community was largely dominated by five species, related to carbohydrate fermentation and VFA production, mainly propionic acid content. Interestingly, an increase in Bacillus and lactic acid microbial species was observed with enzyme and probiotic supplementation, highlighting potential beneficial effects for GI-tract health. This was also supported by the species to species co-occurrence patterns and network 
complexity, suggesting that probiotics and enzyme supplementation contribute to a more stable GI-tract microbiome environment.

\section{Acknowledgements}

We thank the staff of the aquaculture research facilities for their technical support in conducting the experiment and their help with the dissection of the large amount of fish. Ronald Booms, Tino Leffering and Erik van den Brink are gratefully acknowledged for their assistance during laboratory work. This work is part of the Netherlands Organisation for Scientific Research (NWO) research programme "Green aquafeeds" (project number 870.15.100). DuPont Animal Nutrition provided the enzymes and probiotics, analysed the enzyme recovery and colonyforming unit of the probiotics, and partly funded the NWO project. 


\section{Supplementary information}

Table S1 The volatile fatty acids (VFA) content along the gastrointestinal tract of Nile tilapia fed with four experimental diets.

\begin{tabular}{|c|c|c|c|c|c|c|c|c|}
\hline \multirow[t]{2}{*}{ Treatment } & \multicolumn{2}{|c|}{ CON } & \multicolumn{2}{|c|}{ ENZ } & \multirow[b]{2}{*}{ SEM } & \multicolumn{3}{|c|}{$P$-values } \\
\hline & $\mathrm{CON}$ & PRO & CON & PRO & & ENZ & PRO & ENZ*PRO \\
\hline \multicolumn{9}{|c|}{ Volatile fatty acids (mM/L fresh digesta) } \\
\hline \multicolumn{9}{|l|}{ Stomach } \\
\hline Acetic acid & 7.2 & 8.8 & 8.5 & 6.1 & 1.3 & ns & ns & ns \\
\hline Propionic acid & 1.6 & 1.3 & 1.2 & 1.4 & 0.3 & ns & ns & ns \\
\hline Iso butyric acid & nd & $<0.1$ & 0.1 & nd & na & na & na & na \\
\hline Butyric acid & 0.2 & 0.4 & 0.3 & 0.2 & na & na & na & na \\
\hline Iso valeric acid & 0.1 & $<0.1$ & 0.1 & $<0.1$ & na & na & na & na \\
\hline Valeric acid & 0.2 & $<0.1$ & 0.2 & 0.1 & na & na & na & na \\
\hline Total & 9.2 & 10.6 & 10.5 & 7.8 & 1.5 & ns & ns & ns \\
\hline \multicolumn{9}{|l|}{ Proximal } \\
\hline Acetic acid & 9.7 & 16.2 & 12.2 & 15.2 & 1.6 & ns & * & ns \\
\hline Propionic acid & 1.8 & 3.0 & 2.1 & 2.4 & 0.4 & ns & $\#$ & ns \\
\hline Iso butyric acid & nd & nd & nd & nd & na & na & na & na \\
\hline Butyric acid & nd & 0.5 & nd & 0.2 & na & na & na & na \\
\hline Iso valeric acid & $<0.1$ & 0.1 & $<0.1$ & $<0.1$ & na & na & na & na \\
\hline Valeric acid & $<0.1$ & 0.1 & $<0.1$ & $<0.1$ & na & na & na & na \\
\hline Total & 11.5 & 19.8 & 14.4 & 18.0 & 1.7 & ns & $* *$ & ns \\
\hline \multicolumn{9}{|l|}{ Middle } \\
\hline Acetic acid & 10.5 & 8.5 & 11.3 & 9.0 & 1.2 & ns & ns & ns \\
\hline Propionic acid & 1.0 & 0.7 & 0.7 & 0.6 & 0.3 & ns & ns & ns \\
\hline Iso butyric acid & nd & $<0.1$ & nd & nd & na & na & na & na \\
\hline Butyric acid & 0.1 & 0.2 & nd & 0.1 & na & na & na & na \\
\hline Iso valeric acid & 0.1 & $<0.1$ & $<0.1$ & nd & na & na & na & na \\
\hline Valeric acid & 0.1 & 0.1 & $<0.1$ & nd & na & na & na & na \\
\hline Total & 11.7 & 9.4 & 12.0 & 9.7 & 1.3 & ns & ns & ns \\
\hline \multicolumn{9}{|l|}{ Distal } \\
\hline Acetic acid & 10.1 & 9.7 & 10.1 & 9.0 & 0.9 & ns & ns & ns \\
\hline Propionic acid & 0.8 & 0.8 & 1.0 & 0.7 & 0.2 & ns & ns & ns \\
\hline Iso butyric acid & nd & nd & nd & nd & na & na & na & na \\
\hline Butyric acid & 0.1 & 0.1 & nd & nd & na & na & na & na \\
\hline Iso valeric acid & nd & nd & nd & nd & na & na & na & na \\
\hline Valeric acid & nd & nd & nd & nd & na & na & na & na \\
\hline Total & 10.9 & 10.6 & 11.0 & 9.7 & 0.9 & ns & ns & na \\
\hline
\end{tabular}

Notes. CON, no supplementation; ENZ, enzyme (effect) supplementation; PRO, probiotic (effect) supplementation; ENZ*PRO, interaction effect; SEM, standard error of the mean; nd, not detectable; na, not applicable; ns, not significant; $\#, P<0.1 ; *, P<0.05 ; * *, P<0.01$. 
Table S2 The lactic acid content in fresh chyme along the gastrointestinal tract of Nile tilapia.

\begin{tabular}{|c|c|c|c|c|c|c|c|c|}
\hline \multirow[t]{2}{*}{ Treatment } & \multicolumn{2}{|c|}{ CON } & \multicolumn{2}{|c|}{ ENZ } & \multirow[b]{2}{*}{ SEM } & \multicolumn{3}{|c|}{$P$-values } \\
\hline & CON & PRO & CON & PRO & & ENZ & PRO & ENZ*PRO \\
\hline \multicolumn{9}{|c|}{ Lactate (mmol/L fresh digesta) } \\
\hline Stomach & 0.25 & 0.08 & 0.28 & 0.18 & 0.08 & ns & ns & ns \\
\hline Proximal & 0.43 & 0.38 & 0.25 & 0.28 & 0.06 & $*$ & ns & ns \\
\hline Middle & 0.33 & 0.39 & 0.24 & 0.20 & 0.04 & $* *$ & ns & ns \\
\hline Distal & 0.29 & 0.32 & 0.20 & 0.20 & 0.03 & $*$ & ns & ns \\
\hline
\end{tabular}

Notes. CON, no supplementation; ENZ, enzyme (effect) supplementation; PRO, probiotic (effect) supplementation; ENZ*PRO, interaction effect; SEM, standard error of the mean; ns, not significant; $*, P<0.05 ; * *, P<0.01$. 
Table S3 The apparent digestibility coefficient (ADC, \%) along the gastrointestinal tract of Nile tilapia.

\begin{tabular}{|c|c|c|c|c|c|c|c|c|}
\hline \multirow[t]{2}{*}{ Treatment } & \multicolumn{2}{|c|}{$\mathrm{CON}$} & \multicolumn{2}{|c|}{ ENZ } & \multirow[b]{2}{*}{ SEM } & \multicolumn{3}{|c|}{$P$-values } \\
\hline & CON & PRO & CON & PRO & & ENZ & PRO & ENZ*PRO \\
\hline \multicolumn{9}{|l|}{$A D C(\%)$} \\
\hline \multicolumn{9}{|l|}{ Stomach } \\
\hline Dry matter & -0.4 & -0.5 & 2.9 & 0.2 & 0.9 & ns & ns & ns \\
\hline Crude protein & 0.1 & 6.9 & 4.0 & 7.8 & 1.8 & ns & $*$ & ns \\
\hline Ash & 21.8 & 20.1 & 26.7 & 20.9 & 1.3 & ns & ns & ns \\
\hline Phosphorous & 13.0 & 16.9 & 17.8 & 13.9 & 0.9 & ns & ns & \# \\
\hline Calcium & 18.2 & 19.8 & 20.5 & 13.4 & 1.1 & ns & ns & $*$ \\
\hline Copper & 3.3 & 9.2 & 9.3 & 0.8 & 1.7 & ns & ns & $\#$ \\
\hline Magnesium & 20.3 & 11.2 & 25.3 & 18.7 & 3.7 & ns & ns & ns \\
\hline Iron & -20.3 & -31.8 & -29.9 & -26.0 & 0.9 & ns & ns & ns \\
\hline Manganese & 9.0 & 9.6 & 8.7 & -3.4 & 4.8 & ns & ns & ns \\
\hline Zinc & 2.9 & 13.7 & -1.4 & -6.4 & 2.1 & $* *$ & ns & $*$ \\
\hline \multicolumn{9}{|l|}{ Proximal } \\
\hline Dry matter & -53.0 & -85.8 & -37.7 & -46.0 & 6.1 & $*$ & ns & ns \\
\hline Crude protein & -43.8 & -68.3 & -20.1 & -22.2 & 2.3 & $*$ & ns & ns \\
\hline Ash & -95.7 & -140.2 & -74.5 & -77.3 & 8.0 & $*$ & ns & ns \\
\hline Phosphorous & 2.2 & -4.1 & 23.7 & 22.5 & 3.0 & $* *$ & ns & ns \\
\hline Calcium & -12.2 & -18.9 & -10.4 & -15.8 & 1.7 & ns & $\#$ & ns \\
\hline Copper & -137.9 & -216.4 & -160.2 & -171.6 & 15.9 & ns & ns & ns \\
\hline Magnesium & -65.2 & -119.2 & -77.0 & -88.0 & 10.3 & ns & ns & ns \\
\hline Iron & -84.1 & -178.2 & -98.0 & -101.9 & 6.4 & ns & ns & ns \\
\hline Manganese & -19.8 & -36.6 & -31.5 & -44.7 & 17.4 & $*$ & $* *$ & ns \\
\hline Zinc & -48.1 & -51.9 & -59.1 & -67.3 & 2.1 & $*$ & ns & ns \\
\hline \multicolumn{9}{|l|}{ Middle } \\
\hline Dry matter & 33.8 & 28.7 & 34.6 & 36.2 & 1.1 & $\#$ & ns & ns \\
\hline Crude protein & 57.0 & 55.6 & 59.3 & 62.3 & 0.9 & $*$ & ns & ns \\
\hline Ash & -20.0 & -27.0 & -14.8 & -10.9 & 1.7 & $* *$ & ns & ns \\
\hline Phosphorous & 27.8 & 27.5 & 41.8 & 44.6 & 1.5 & $* * *$ & ns & ns \\
\hline Calcium & 7.3 & 10.6 & 15.7 & 16.2 & 1.7 & $\#$ & ns & ns \\
\hline Copper & 2.2 & 2.9 & -5.8 & -1.4 & 2.5 & ns & ns & ns \\
\hline Magnesium & 12.5 & 9.4 & 13.7 & 17.0 & 1.5 & ns & ns & ns \\
\hline Iron & -22.9 & -41.0 & -36.0 & -56.9 & 1.0 & ns & $*$ & ns \\
\hline Manganese & -5.5 & -23.2 & -11.0 & -22.6 & 4.3 & ns & $* *$ & ns \\
\hline Zinc & -14.2 & -14.5 & -20.1 & -22.5 & 1.8 & $* *$ & ns & ns \\
\hline
\end{tabular}

Notes. CON, no supplementation; ENZ, enzyme (effect) supplementation; PRO, probiotic (effect) supplementation; ENZ*PRO, interaction effect; SEM, standard error of the mean; ns, not significant; $\#, P<0.1 ; *, P<0.05 ; * *, P<0.01 ; * * *, P<0.001$. 


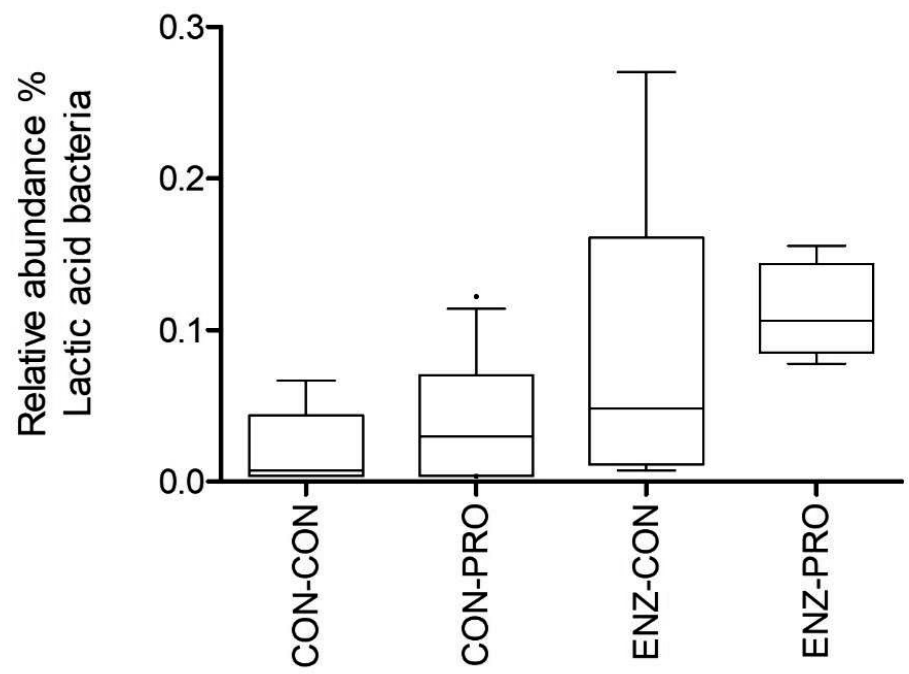

Figure S1 Relative abundance of lactic acid bacteria in the Nile tilapia distal gut. CONCON, no enzymes or probiotics added; CON-PRO, probiotics added; ENZ-CON, enzymes added; ENZ-PRO, enzymes and probiotics added.

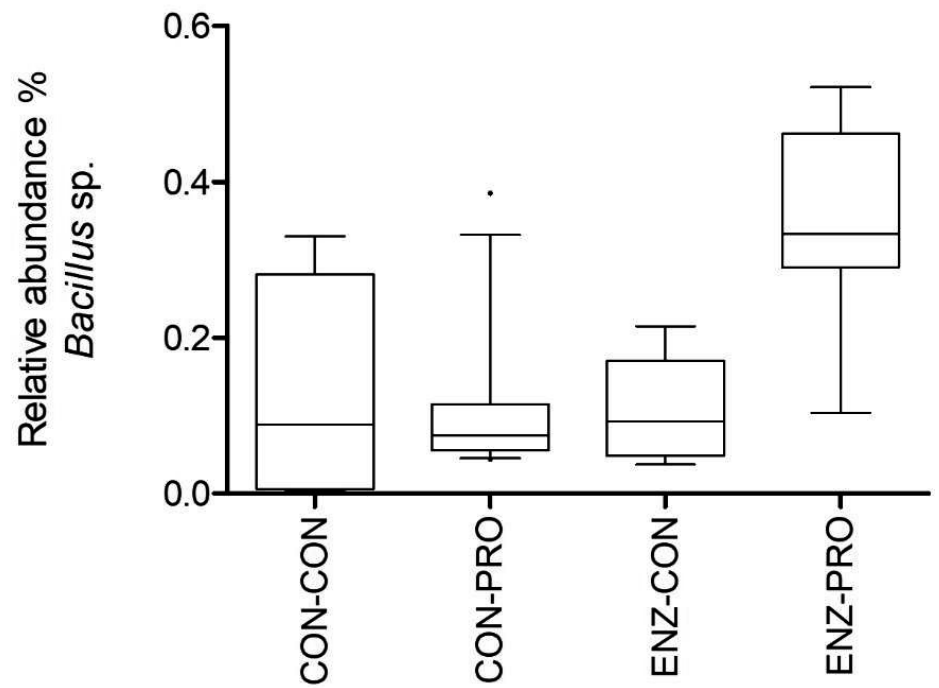

Figure $\mathbf{S 2}$ Relative abundance of Bacillus species in the Nile tilapia distal gut. CON-CON, no enzymes or probiotics added; CON-PRO, probiotics added; ENZ-CON, enzymes added; ENZ-PRO, enzymes and probiotics added. 

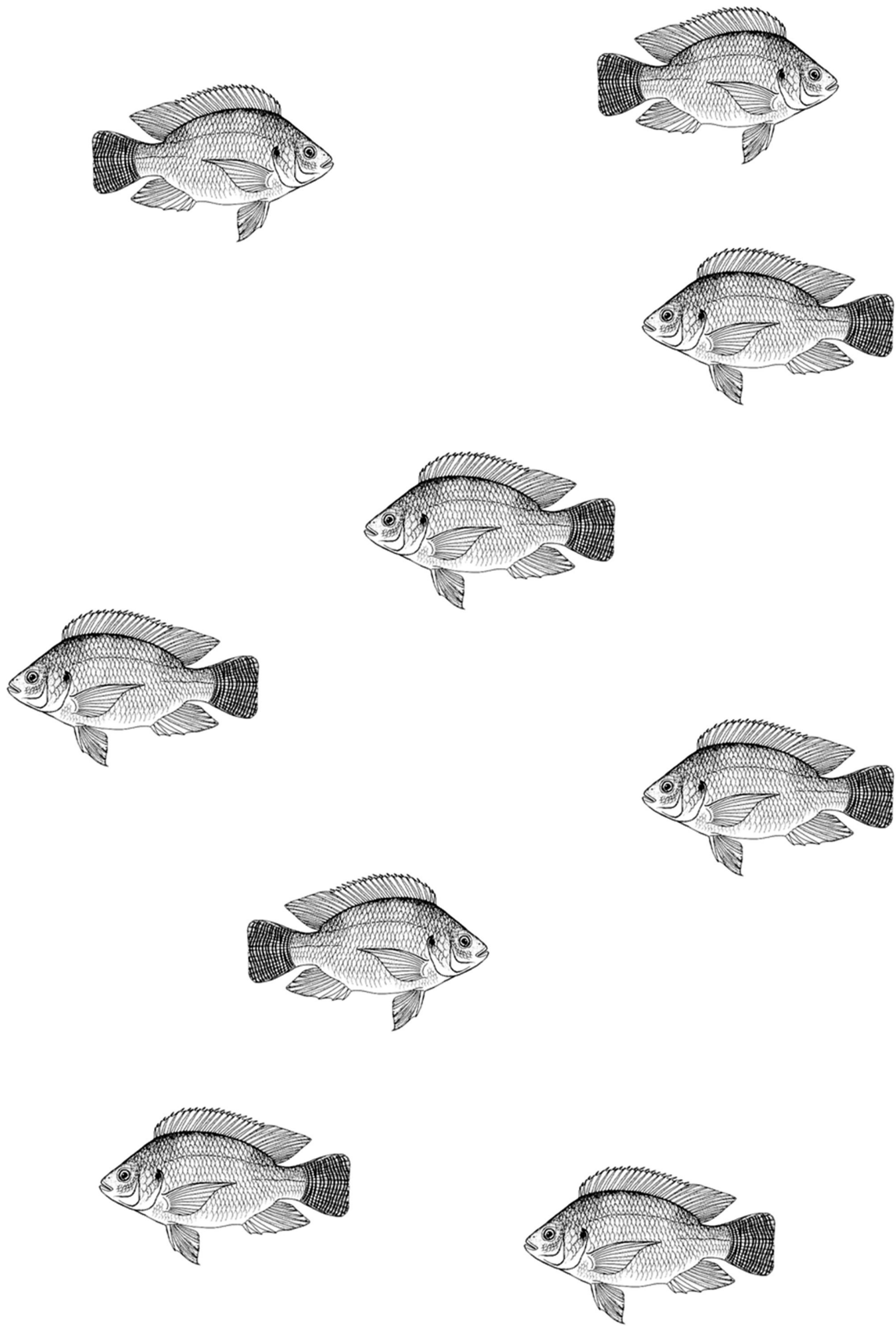


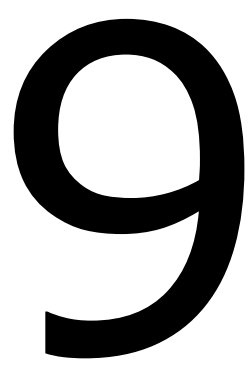

General discussion 


\section{Introduction}

This thesis investigated whether the nutritional value of low quality aquafeeds could be improved by dietary supplementation with exogenous enzymes and probiotics. Tilapia was used as a model species. The objective was predominantly to alleviate the negative effects of dietary non-starch polysaccharides (NSP) and phytates on nutrient digestibility and growth. The ultimate aim is to enable the use of low-quality plant-based ingredients in aquafeeds, thereby contributing to the circularity of the aquaculture sector. This chapter reflects on the results and main conclusions from previous chapters. It is assessed whether enzymes and probiotics can be supplemented, to low quality feeds, to successfully improve nutrient digestibility and utilisation, as well as growth performance. Firstly, the effectiveness of phytase, NSP-degrading enzymes and probiotics, in improving diet quality, is reflected upon. Secondly, the interactions between enzymes (mutually) and probiotics are outlined. Finally, insight is provided for future research, including implications of research outcomes for current and future fish and aquafeed production.

\section{Phytase}

\section{Impact of phytase on phosphorus availability}

Phytase was supplemented to a control diet across all the experiments, either on its own or in combination with xylanase. There was always an improvement in phosphorus $(P)$ availability with phytase supplementation, regardless of whether it was supplemented alone or in combination with other enzymes (xylanase and $\beta$ glucanase) (Table 1). In order to check for the effect of enzymes across experiments, correlations between parameters, like phytase dose and change in $\mathrm{P}$ availability, were explored with Pearson's correlation coefficient; if significant, equations were calculated. In order to make a comparison across studies, the effect of enzymes was always calculated relative to the control diet (delta, $\Delta$ ). The probiotic treatment (Chapter 7) and the $\beta$-glucanase treatments (Chapter 6) were excluded from the dataset, because they were only included at one dose and not across all diets.

Large differences in the increase in $\mathrm{P}$ availability (i.e., ADC of P) were observed between studies and diets with enzyme supplementation. The phytase dose, when below optimum, can have an dose-response effect on the $\mathrm{P}$ availability (Portz and Liebert, 2004; Liebert and Portz, 2005). In this thesis, the aim was to have a phytase activity of $1000 \mathrm{FTU} / \mathrm{kg}$ diet, which is at the higher end of the optimal range (750-1000 FTU/kg), as reported for tilapia by Liebert and Portz (2005). Although the targeted phytase activity was $1000 \mathrm{FTU} / \mathrm{kg}$, large differences in phytase activity were recorded between studies (621-1175 FTU/kg). The relationship between enzyme dose ( $\Delta$ phytase) and $\mathrm{P}$ availability, between test and control diets ( $\triangle \mathrm{P}$ availability in $\%)$, showed a positive correlation $(P<0.05)$. However, the actual $\Delta$ phytase explained only $29.3 \%$ of the variation in $\Delta P$ 
availability (Figure 1a). For example the lowest $\Delta$ phytase was $621-667 \mathrm{FTU} / \mathrm{kg}$ (Chapter 4), with an associated increase in P availability of $13-14 \%$. This was even higher than the increase in $P$ availability observed where higher doses of phytase were received (Chapter $\mathbf{3}$ and $\mathbf{6}$ ) (Table 1).

Table 1 Selection of main parameters related to the enzyme response and to the experimental design (used to check for correlations across chapters).

\begin{tabular}{lcccccccc}
\hline & $\begin{array}{l}\text { P con-diet } \\
\text { (g/kg DM) }\end{array}$ & $\begin{array}{l}\Delta \text { phytase } \\
\text { (FTU/kg) }\end{array}$ & $\begin{array}{l}\Delta \text { xylanase } \\
(\mathbf{U} / \mathbf{k g})\end{array}$ & $\begin{array}{l}\text { P ADC con- } \\
\text { diet (\%) }\end{array}$ & $\begin{array}{l}\Delta \mathbf{P} \\
\text { ADC (\%) }\end{array}$ & $\begin{array}{l}\text { NSP ADC } \\
\text { con-diet (\%) }\end{array}$ & $\begin{array}{l}\Delta \text { NSP } \\
\text { ADC (\%) }\end{array}$ & $\begin{array}{l}\Delta \text { growth } \\
(\mathbf{g} / \mathbf{d})\end{array}$ \\
\hline Chapter 3 & 9.7 & 860 & 3461 & 76.6 & 3.2 & -3.1 & 3.0 & -0.01 \\
& 9.0 & 926 & 3708 & 63.7 & 9.5 & 17.1 & 8.0 & 0.10 \\
& 9.5 & 991 & 3936 & 60.8 & 7.8 & 18.1 & 3.5 & 0.06 \\
& 7.6 & 741 & 3732 & 76.8 & 1.9 & 31.7 & 1.2 & -0.02 \\
\hline Chapter 4 & 9.8 & 662 & 0 & 51.4 & 13.9 & 17.9 & 4.6 & 0.11 \\
& 9.8 & 621 & 6348 & 51.4 & 12.8 & 17.9 & 2.5 & 0.09 \\
& 11.8 & 667 & 0 & 45.0 & 13.8 & 25.3 & 4.4 & 0.09 \\
& 11.8 & 667 & 6844 & 45.0 & 14.1 & 25.3 & 11.3 & 0.13 \\
\hline Chapter 5 & 9.5 & -29 & 4228 & 39.3 & 0.0 & - & - & 0.02 \\
& 9.5 & 995 & 0 & 39.3 & 26.8 & - & - & 0.12 \\
& 9.5 & 1147 & 4241 & 39.3 & 29.5 & - & - & 0.22 \\
\hline Chapter 6 & 9.5 & 960 & 3655 & 72.6 & 4.1 & 37.3 & -2.7 & -0.02 \\
& 9.5 & 0 & 2987 & 72.6 & 0.7 & 37.3 & 1.3 & 0.02 \\
& 9.5 & 1175 & 0 & 72.6 & 6.6 & 37.3 & 1.0 & 0.02 \\
\hline Chapter 7 & 11.0 & 667 & 6844 & 41.2 & 23.6 & 28.6 & 9.8 & 0.25 \\
\hline
\end{tabular}

Notes. $\Delta$, reflects the difference in the respective parameters between test and control diets; condiet, control diet; P, phosphorus; ADC, apparent digestibility coefficient; NSP, non-starch polysaccharides.
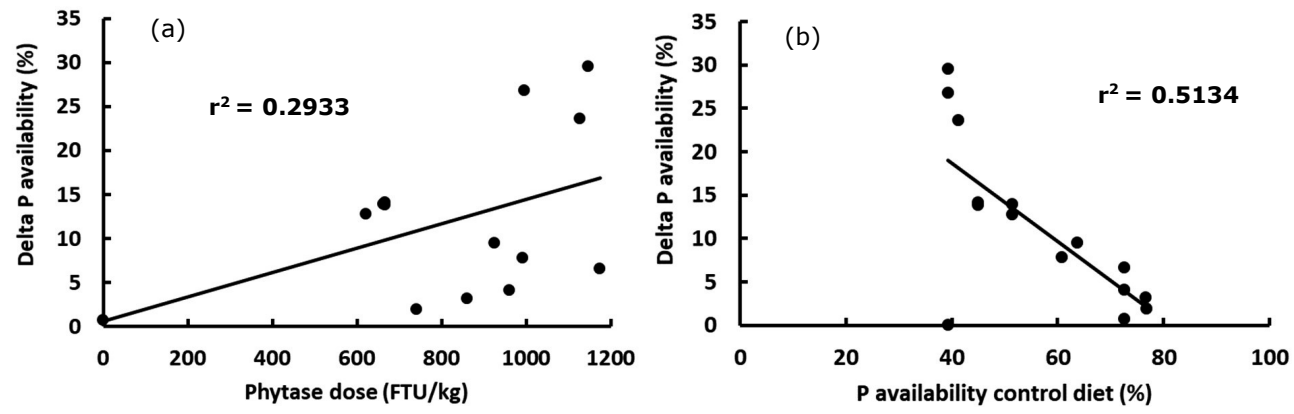

Figure 1 (a) Relationship between the phytase dose (FTU/kg) and the $\Delta$ in phosphorous $(\mathrm{P})$ availability (percentage points, \%); (b) relationship between the phosphorous ( $P$ ) availability of the control diet and the increase in $\mathrm{P}$ digestibility (percentage points, \%) with phytase supplementation.

Both the level of phytase supplemented and the characteristics of the control diet play a major role in how enzyme supplementation impacts $\Delta \mathrm{P}$ availability. Across 
studies it was observed that the improvement in $\mathrm{P}$ availability, with enzyme supplementation to the control diet, was lower when the $\mathrm{P}$ availability of the control diet was already high (Figure 1b). For example, in chapter 3, two control diets (without phytase; the reference diet and the citrus pulp diet) had a $\mathrm{P}$ availability of about $77 \%$. Phytase supplementation of these diets gave only a marginal increase in $\mathrm{P}$ availability. These two control diets had the lowest calculated phytate content, compared to the other diets in chapter 3. In general, higher available $\mathrm{P}$ levels in the control diets (without enzymes supplemented) most likely correlates with lower levels of phytate, as phytate is considered indigestible. Therefore, there is potentially less to gain with phytase supplementation when the level of phytate is low, as this results in a lower response. Across all chapters, the $P$ availability of the control diet explained $51 \%$ of the variation of the $\Delta$ phytase response on $P$ availability $(P<0.01$; Figure $1 \mathrm{~b})$.

Figure 1 shows that xylanase supplementation without phytase does not improve the $P$ availability (points on and close to the $x$-axis). In Figure $1 \mathrm{a}$, the points without phytase (xylanase only) significantly contribute to the observed correlation (no effect without phytase). On the contrary, in Figure 1b the lack of response in diets without xylanase reduces $r^{2}$. Combining the enzyme dose, and $P$ availability of the control diet into an extended model, gives the best fit $\left(r^{2}=0.967\right)$ for predicting the $\Delta \mathrm{P}$ availability, resulting in the following formula:

$\Delta \mathrm{P}$ availability $=1(\mathrm{SE} 4.3)-0.006(\mathrm{SE} 0.075) \times X 2-0.059(\mathrm{SE} 0.009) \times X 1 \times X 2$

Where, $X 1$ is the ADC (\%) of the control diet and, $X 2$ the $\Delta$ phytase dose (100 $\mathrm{FTU} / \mathrm{kg}$ ). Therefore, $\Delta \mathrm{P}$ availability is dependent on the control diet and the presence and dose of phytase. This relationship, using the above formula, is displayed in Figure 2. It should be noted that beyond the optimal phytase dose, $\Delta$ $P$ availability will not further increase (plateau). Liebert and Portz (2005) stated that the optimal phytase dose is $750-1000$ FTU/kg for tilapia. However, as suggested by the above equation and the data in Figure 1, it seems that the quality of the diet (most likely the phytate content) strongly influences the optimal dose of phytase.

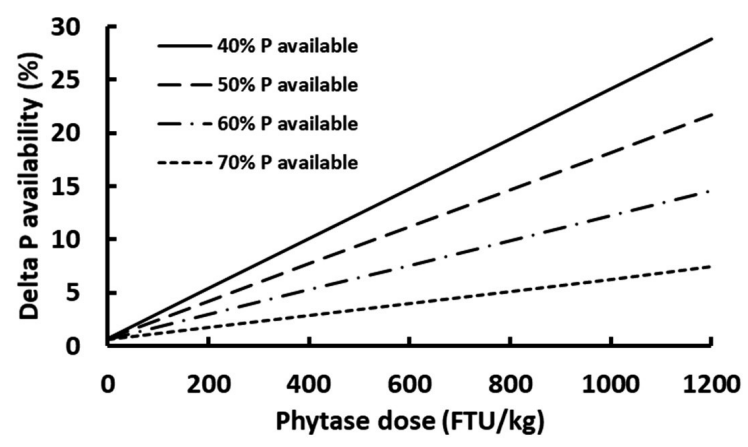

Figure 2 Relationship between the available (\%) phosphorous (P) level of the control diet (no enzymes) and phytase dose and its effect on $\Delta \mathrm{P}$ availability. 
The structure of phytate, an inositol ring with six phosphate esters, is always the same. However, whether phytate is available for hydrolysis by phytase might be dependent on its location in the plant. In legumes, for example, phytate is distributed throughout the whole seed, whereas in cereals, phytate is mainly concentrated in the aleurone layer (Skoglund et al., 2009; Kumar et al., 2012). It is likely that phytate located in the endosperm, for example, which mainly consists of highly digestible starch, is more accessible to phytase, compared to phytate that is bound or entrapped in the cell wall. Therefore, the choice of plant-based ingredient(s) may influence the level of improvement in P availability with phytase supplementation. Measuring phytate in both the feed and faeces would allow for the improvement in availability of phytate-bound $P$ to be quantified. This also would enable the influence of diet-related factors on the breakdown rate of phytate to be determined. Measuring phytate in the control treatments (no enzymes) and faeces could provide an indication as to whether phytate is completely inert to digestion, or if part of the phytate can be broken down endogenously, e.g. with the help of the intestinal microflora.

The effect of phytase was shown to be dependent on the level of available $P$ in the control diet (Figure 1b). In the future, increasing levels of low quality plant-based ingredients in aquafeeds may lead to an increase in the level of phytate in the diet, thus having a negative impact on diet quality overall. Therefore, when using more plant by- and co-products in aquafeeds it will be beneficial to use phytase to enhance $P$ availability. As the increase is $P$ availability is partly dependent on the supplemented dose of phytase, it should be sufficient to ensure an optimal increase in $P$ availability. The level of phytate could potentially affect the optimal phytase dose, however this requires further research (i.e. measuring phytate digestibility).

\section{Effect of phytase on nutrient digestibility}

Phytase supplementation (alone or in combination with xylanase) improved not only $\mathrm{P}$ availability, but also dry matter, protein, fat energy, carbohydrate, NSP, ash, and calcium ( $\mathrm{Ca}$ ) and magnesium digestibility. However, only the effect of phytase on $\mathrm{P}$ and $\mathrm{Ca}$ availability and ash digestibility was observed across all studies. Phytate has also been shown to influence the availability and digestibility of other nutrients; thus with lower levels of phytate, the negative effects will be partly neutralised. It is generally accepted that phytate mainly reduces fish growth by reducing mineral bio-availability (Riche and Brown, 1996; Papatryphon et al., 1999; Forster et al., 1999) and protein digestibility (Riche and Garling, 2004; Portz and Liebert, 2004; Liebert and Portz, 2007). Mainly at a low pH, phytic acid will chelate to di- and trivalent minerals (and thus ash fraction), forming stable complexes. The amount of $\mathrm{Ca}$ in fish feeds is high, compared to other cations, therefore phytic acids will chelate mainly with $\mathrm{Ca}$. In line with the improvement in $P$ availability, phytase improved Ca availability throughout all experiments, with a positive correlation between the phytase dose and $\Delta$ Ca availability $(P<0.01)$. The correlation between the phytase dose and $\Delta$ Ca availability is even stronger, compared to $\Delta \mathrm{P}$ availability $\left(r^{2}=0.524\right.$ vs. $\left.r^{2}=0.293\right)$. 


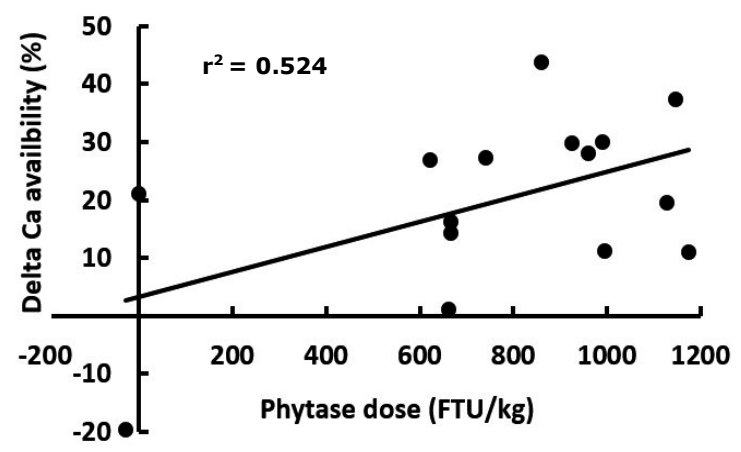

Figure 3 Relationship between the phytase dose (FTU/kg) and the $\Delta$ in calcium (Ca) availability (percentage points, \%).

The effect of enzyme supplementation, including phytase, is inconsistent for macronutrients (fat; protein; carbohydrate, including NSP). As with minerals, phytate can form complexes with proteins, or indeed with both proteins and minerals, thus forming ternary complexes (phytate-mineral-protein), which can lead to reduced protein digestibility (Rich and Garling 2004; Kumar et al., 2012; Dersjant-Li et al., 2015). The presumed negative effect of phytate on protein digestibility was in line with the improvements observed in this thesis (Chapter 5) and with other tilapia studies (Portz and Liebert, 2004; Liebert and Portz, 2007). Phytase alone did not improve protein digestibility (Chapter $\mathbf{3}$ ). As in this thesis (Chapter 5), improvement in fat digestibility has previously been observed with phytase supplementation (Hussain et al., 2011), however a robust explanation remains absent. Several direct and indirect mechanisms could be involved in the improvement of fat digestibility with phytase. For instance, it could be that increasing levels of available $\mathrm{Ca}$ in the gut, which is known to increase lipase activity (Fave et al., 2004), leads to increased fat digestibility. This thesis shows the ability of phytase to increase carbohydrate digestibility; this may be linked to an increase in NSP digestibility, which leads to an overall improvement in carbohydrate digestibility. The ability of phytase to enhance carbohydrate or NSP digestibility has not been described before, most likely because it is seldom measured in fish. With an $\Delta$ of $11.3 \%$ for NSP digestibility, the contribution of phytase to enhancing NSP digestibility was significant (Table 1). Exactly how phytase improves NSP digestibility remains unclear. It could be speculated that the feed matrix changes with phytase; for example, by breaking down phytate attached to the cell wall (aleurone layer), thus improving the accessibility of NSP for endogenous digestion. It was observed that the improvement (although small) in digestibility of fat, protein, carbohydrate and NSP, negatively correlates with $\mathrm{P}$ availability in the control treatments (no enzymes) (Figure 4). In other words, the impact of enzyme(s) supplementation on the $\Delta$ digestibility of all nutrients increases with decreasing $P$ availability (i.e. $P$ ADC) in the control diets. As previously described, low $P$ availability in the control diet can be linked to a lower diet quality (i.e. higher dietary levels of phytate and NSP). The $\Delta$ digestibility of both crude protein and carbohydrate (including NSP), correlates positively with the 
dietary NSP content $(P<0.05)$. This highlights the fact that with lower diet quality (higher levels of NSP and phytate), there is more to gain from enzyme supplementation. In all probability, the larger decrease in antinutritional factors (ANFs) (NSP and phytate), in the "lower" quality diets, when supplemented with enzymes, causes a greater improvement in digestibility for those macronutrients.

It should be noted that although the $\Delta$ digestibility (\%) is correlated with the $\mathrm{P}$ availability (\%) of the control diet (Figure 4), it is hard to separate what can be attributed to phytase or xylanase. Only in 4 out of 15 data points was phytase supplemented without xylanase. Moreover, the improvement in digestibility was not related to the supplementation dose of phytase for any of the macro-nutrients. The phytase dose was not correlated with the $\Delta$ digestibility of macro-nutrients. This, in combination with Figure 4, suggests that in the current thesis experiments, the quality of the control diets was more important in determining the impact of enzyme supplementation, than the dose. It could be hypothesised that smaller doses of enzymes are already sufficient to counteract the negative effects of, for example, phytate and NSP on the digestibility of macro-nutrients in tilapia. This requires further research.
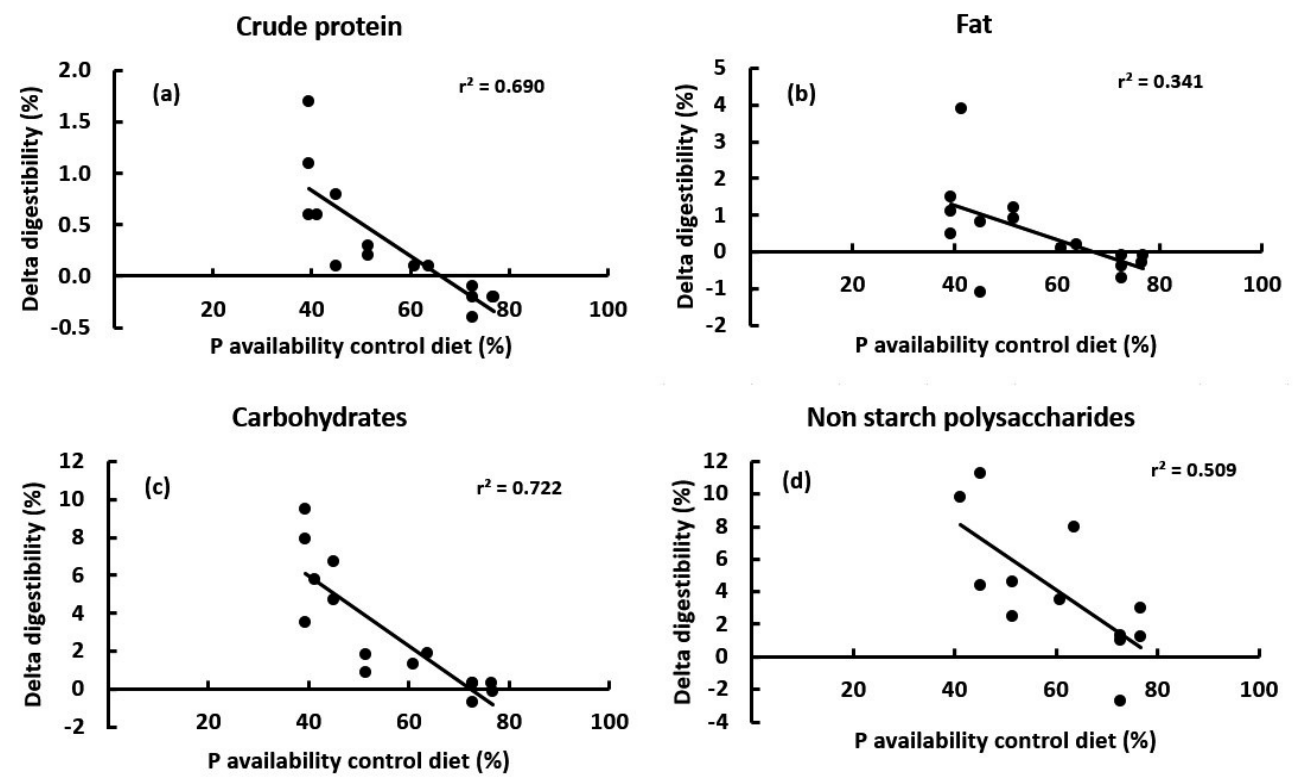

Figure 4 relationship between the phosphorous $(P)$ availability of the control diet and the (a) $\Delta$ crude protein digestibility, (b) $\Delta$ fat digestibility, (c) $\Delta$ carbohydrate digestibility, and (d) $\Delta$ non-starch polysaccharide digestibility. 


\section{Phosphorus requirements}

In all studies performed in this thesis, improving $P$ availability via phytase supplementation resulted in a stronger than anticipated effect on growth. All control diets were formulated to have an available $P$ content of at least $4.5 \mathrm{~g} / \mathrm{kg}$ dry matter (DM) feed. Except for two dietary treatments without phytase (Chapter 5), all treatments met this target level. The $\Delta$ available $\mathrm{P}$ had a strong correlation with $\Delta$ growth (Figure 5). Fish have the ability to use $\mathrm{P}$ stored in bones (remineralisation) and scales for ATP synthesis and growth (Lall and McCrea, 2007). This could potentially lead to a delayed growth response under conditions of low dietary P. Despite this, the relationship between dietary available $P$ and weight gain fits best to a linear broken line regression plateau model (Prabhu et al. 2013). This suggests that the response to limiting dietary $P$ levels for growth is rather abrupt. Broken-line models are commonly fitted to nutritional response data. Hereby, the break point (slope of the line is zero) indicates the minimal requirement is reached. However, the results from the current study show that the breakpoint is not reached (Figure 5), and therefore from this data, a minimum available $P$ level for growth cannot be given. The strong increase in growth with available $P$ levels above $4.5 \mathrm{~g} / \mathrm{kg} \mathrm{DM}$, clearly demonstrates that $4.5 \mathrm{~g}$ of available $\mathrm{P} / \mathrm{kg} \mathrm{DM}$ is insufficient to support maximal growth. At the highest $\Delta$ available $\mathrm{P}$ $(2.8 \mathrm{~g} / \mathrm{kg}$ DM diet), $\Delta$ growth $(\mathrm{g} / \mathrm{d})$ did not reach a plateau. This indicates that, to sustain maximal growth in Nile tilapia, available $\mathrm{P}$ needs to be greater than 7.0 $\mathrm{g} / \mathrm{kg}$ DM of feed. This is in line with the recently estimated available $P$ requirements of $8.6 \mathrm{~g} / \mathrm{kg}$ feed by Yao et al. (2014). The current stated dietary P requirement for tilapia by the NRC (2011) is $4.0 \mathrm{~g} / \mathrm{kg}$ feed (total P). Prabhu et al. (2013) observed an available $P$ requirement for growth of $4.7 \mathrm{~g} / \mathrm{kg}$ DM feed by meta-analysis, however, this requirement is across species (excluding rainbow trout). The large improvement found in growth within this study, with increasing available P levels (above the stated requirement for tilapia, NRC, 2011; and across species, Prabhu et al., 2013), indicates that the current recommendations are incorrect for Nile tilapia, as also supported by data from Yao et al. (2014).

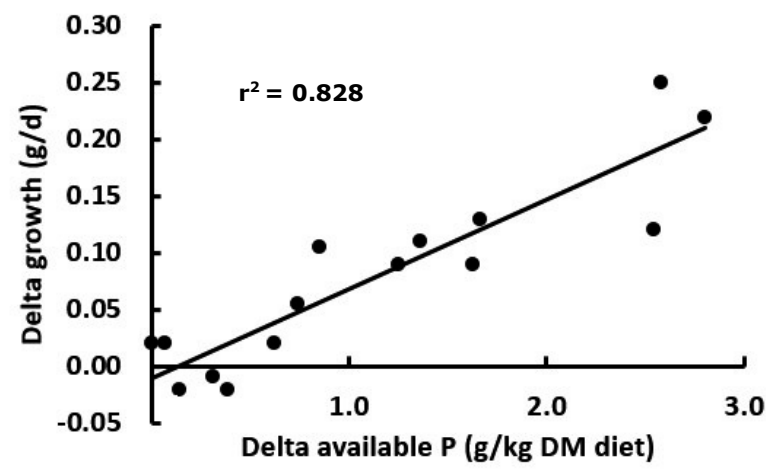

Figure 5 relationship between the $\Delta$ available dietary phosphorus $(\mathrm{g} / \mathrm{kg} \mathrm{DM})$ and the $\Delta$ growth $(g / d)$. 
In line with the increase in growth, there were considerably higher levels of whole body $P$ content in phytase supplemented diets $(6 \mathrm{~g} / \mathrm{kg} P$ fresh weight), compared to the control diets $(4.8 \mathrm{~g} / \mathrm{kg} P$ fresh weight) (Figure 6; Chapter 5 and 7 ). The maximal values (Chapter $\mathbf{5}$ and $\mathbf{7}$ ) were equal, which suggests that the maximal whole body $\mathrm{P}$ content is approximately $6 \mathrm{~g} / \mathrm{kg}$ (on fresh basis) for Nile tilapia. Also, this value is higher than the only value currently published in the literature for Nile tilapia (Yao et al., 2014). Available P requirements were estimated to be $8.6 \mathrm{~g} / \mathrm{kg}$ feed and the (maximal) whole body $P$ content was $5.2 \mathrm{~g} / \mathrm{kg}$ fresh weight in fish fed diets at and above the minimal $P$ requirement (Yao et al., 2014).

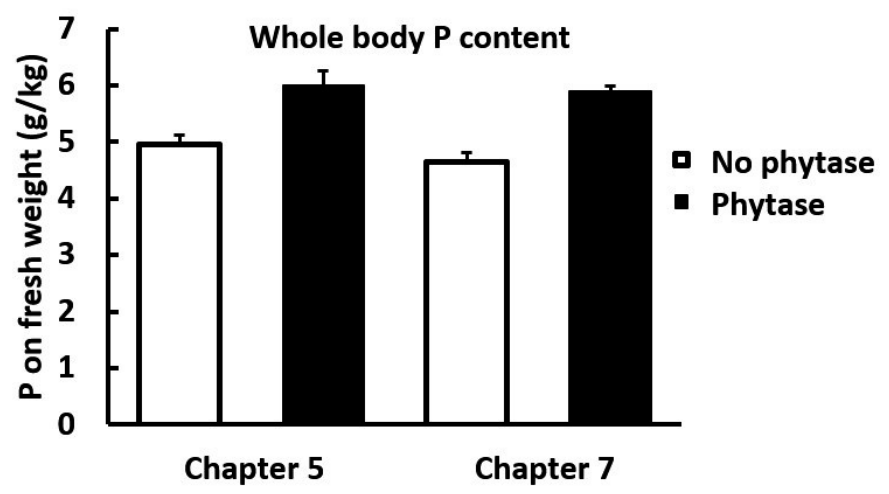

Figure 6 average whole body phosphorus $(P)$ content ( $\mathrm{g} / \mathrm{kg}$ fresh weight) for fish fed diets with and without phytase (chapter 5 and 7 ), error bars represent the standard deviation.

In this thesis, the $\mathrm{P}$ efficiency remained high despite increasing levels of available $P$. Normally, branchial and urinary $P$ losses increase when available $P$ increases above the minimal $P$ requirement, which results in a reduced $P$ efficiency (percentage retained $\mathrm{P}$ of the available P) (Rodehutscord, 1996; Rodehutscord et al., 2000). The dietary treatments with phytase supplementation (Chapter 5), compared to the control diets, had an increased $\mathrm{P}$ availability but no decline in $\mathrm{P}$ efficiency $(90.5 \%)$. The $P$ efficiency even increased with increasing levels of available P (Chapter 7), due to dietary phytase supplementation (from $67.5 \%$ up to $80.9 \%$ ). These observations support the aforementioned assumption that the current $\mathrm{P}$ level guidelines for Nile tilapia are an underestimation.

In summary, dietary supplementation of phytase increases the P availability in Nile tilapia. However, the magnitude of this improvement is dependent on the diet quality (i.e. level of phytate). Besides improving $\mathrm{P}$ availability, phytase can also improve the digestibility of other nutrients as well (i.e. Ca, fat, crude protein, carbohydrate and NSP). Considering the strong effect of increasing available $\mathrm{P}$ levels on growth and whole body $\mathrm{P}$ content, the $\mathrm{P}$ requirements for Nile tilapia need careful reconsideration in order to prevent impaired growth and to optimise feed efficiency. 


\section{NSP-degrading enzymes}

\section{Endogenous NSP digestibility}

Although considered indigestible, the current meta-analyses (Chapter 2) and a few other studies using tilapia (Amirkolaie et al., 2005; Leenhouwers et al., 2007; Haidar et al., 2016) clearly demonstrate the partial digestion of NSP. The NSP digestibility averaged $24.3 \%$ across studies. Although this is relatively low (24.3\%), NSP digestibility has been shown to contribute to energy digestibility. The value measured is most likely related to fermentation, whereby volatile fatty acids (VFA) are produced (Bergman 1990; Metzler-Zebeli et al., 2010). Like in studies by Amirkolaie et al. (2005) and Leenhouwers et al. (2007), concentrations of VFA were measured along the gastrointestinal tract (GI-tract) (Chapter 7), indicating the occurrence of fermentation in the GI-tract. In line with the results of the current study (Chapter 2), all other studies indicate NSP digestibility of the control treatments without enzymes (endogenous), with the exception of the reference diet $(-3.1 \%)$, which is low in NSP (Chapter 3 ). The NSP fraction was not analysed, but calculated from the measured DM, ash, crude protein, fat and starch and sugars. If NSP digestibility is $0 \%$, then a small variation in measuring DM, etc. may induce negative values, especially when the NSP fraction is low, as in the reference diet (Chapter 3). An additional reason could be an incorrect Jones factor of 6.25 for calculating crude protein from analysed nitrogen (N) content. A lower Jones value will give an underestimation of NSP in the diet, leading to a negative value for NSP digestibility. In this thesis, the endogenous NSP digestibility of all the control diets (without enzyme supplementation) averaged $21.2 \%$ (SD \pm 11.3 ), which is highly comparable to the average of the meta-analyses (Chapter 2). Also in line with this meta-analysis, the variation in NSP digestibility was large between the control diets in this thesis, ranging from $-3.1 \%$ to $37.3 \%$ (Table 1 ). This thesis shows that part of the variation in endogenous NSP digestibility can be explained by the NSP composition (Chapter $\mathbf{3}$ ) and the dietary level of NSP (Chapter 4). Hereby it is shown that the endogenous NSP digestibility is higher when the diet is rich in pectins, compared to diets rich in either hemicellulose or cellulose. With increasing NSP levels, the endogenous NSP digestibility increased (Chapter 4), while the NSP composition remained comparable. However, across studies, there was no correlation between dietary NSP level and endogenous NSP digestibility $(P>0.1)$. This is most likely because the NSP composition is the main factor affecting endogenous NSP digestibility. However, there are still unknown factors affecting NSP ADC in tilapia, as shown in one of the control diets where, with storage time, the NSP ADC increased (Chapter 6).

\section{Xylanase and $\beta$-glucanase}

The main effects of $\beta$-glucanase and its interaction with phytase and xylanase were tested (Chapter 6). $\beta$-glucanase is the second most utilised carbohydrase globally, after xylanase (Adeola and Cowieson, 2011; Castillo and Gatlin, 2015). Despite this, the main effect of $\beta$-glucanase (i.e. without the presence of other 
enzymes) on improving diet quality had never before been tested. However, $\beta$ glucanase has been used in various studies, often in combination with xylanase (Lin et al., 2007; Ogunkoya et al., 2006; Stone et al., 2003; Yildrim and Turan, 2010). $\beta$-glucanase alone, or in combination with xylanase and/or phytase, did not affect nutrient digestibility or any growth parameters (Chapter 6). Besides the absence of an effect of $\beta$-glucanase, there was also only a weak response to phytase and xylanase, with no influence on growth. Comparison of the results with those of the wheat bran diet in chapter 3, which was the same diet as in chapter 6, shows that enzyme supplementation to the control diet resulted in improved growth and nutrient digestibility (Chapter $\mathbf{3}$ ). However, in chapter $\mathbf{3}$ this control diet (no enzymes) had lower growth and nutrient digestibility (compared to chapter 6) and therefore there was little to improve when enzymes were supplemented in chapter $\mathbf{6}$. This might also have caused the absence of a $\beta$ glucanase effect. How diet related factors affected the control diet over time, and thus the repeatability, remains unclear. Therefore, the results of this single experiment are inadequate to draw conclusions on the effectiveness of $\beta$-glucanase in enhancing diet quality. Further research on the potential impact of $\beta$-glucanase is advisable, especially for low quality diets (i.e. low NSP digestibility and P availability).

Xylanase can positively affect nutrient digestibility and growth (Chapter 4 and 5). However, across studies there is not a clear dose response of xylanase supplementation, despite the considerable variation in the applied doses between studies (Table 1). Likewise, where most studies show improved growth with xylanase supplementation across fish species (Dalsgaard et al., 2012; Jiang et al., 2014; Hassaan et al., 2019; Jin et al., 2020), some do not find an effect on growth at all (Wallace et al., 2016). A large range of xylanase doses were used, ranging from 2987 to $6844 \mathrm{U} / \mathrm{kg}$ (Table 1). Despite the large range of doses, there was no effect on the $\Delta$ digestibility of any of the measured nutrients. On the contrary, both in grass carp (Jin et al. 2020) and Jian carp (Jiang et al., 2014), an optimum xylanase dose was recorded $(\mathrm{U} / \mathrm{kg}$ ), of around 1530 and $1260 \mathrm{U} / \mathrm{kg}$, respectively. Above this optimum, the growth rate decreased. Although xylanase targets xylans, which is part of the NSP fraction, the presumed increase in NSP digestibility (Chapter 5) is greater with phytase. Indeed, phytase was shown to have a strong effect on NSP digestibility (Chapter 4). Furthermore, phytase had a stronger effect on all growth parameters and led to higher nutrient digestibility of all parameters, compared to xylanase (Chapter 5). As both phytase and xylanase affect $\Delta$ NSP digestibility, it is difficult to relate changes in $\triangle$ NSP digestibility to either phytase, xylanase or both, when provided together. The $\Delta$ NSP digestibility ranged from $2.7 \%$ up to $11.3 \%$. The treatments with the highest $\Delta(+5)$, were all a combination of both phytase and xylanase, which suggests an additive or even synergistic effect between the two on NSP digestibility. With greater NSP digestibility and rising levels of dietary NSP, the importance of NSP as an energy source is set to increase (Chapter 2). 
The dietary NSP level ( $\mathrm{g} / \mathrm{kg} \mathrm{DM})$ and the increase in NSP digestibility between test and control diets ( $\triangle$ NSP digestibility in \%) showed a positive correlation $(P<0.05)$. However, the dietary NSP level only explains $36.7 \%$ of the variation in $\triangle$ NSP digestibility (Figure 7). This is in all probability related to the effect NSP composition has on $\triangle$ NSP digestibility. As there is a large variety in types of NSP, it is important that enzyme(s) supplementation is complementary to the diet composition (Officer, 2000). For example, the NSP level of the test diets varied slightly as follows: $13.2 \%$ (wheat bran diet); $17.9 \%$ (sunflower meal diet); and $17.4 \%$ (citrus pulp diet) (Chapter 3 ). In contradiction to the observed correlation (Figure 7 ), the $\Delta$ NSP digestibility was highest for the wheat bran diet $(8.0 \%)$, and lowest for the sunflower meal (3.5\%) and citrus pulp diets (1.2\%). This observed difference was in line with the expectation that wheat bran, being relatively rich in hemicellulose, is most affected when xylanase is supplemented; as xylanase hydrolyses the xylan backbone of arabinoxylans, which is part of the hemicellulose fraction (Collins et al., 2005). With each \% increase in $\Delta$ NSP digestibility, the absolute amount of NSP digested ( $\mathrm{g} / \mathrm{kg}$ DM diet) increases at a much higher rate for diets high in NSP. Therefore, the positive correlation between the dietary NSP level and $\triangle$ NSP digestibility (Figure 7), underlines the observation that the enzyme dose is not limiting. However, why $\triangle$ NSP digestibility increases with increased dietary NSP levels is unclear, as the NSP composition is expected to be the main driver of $\triangle$ NSP digestibility and not the NSP level.

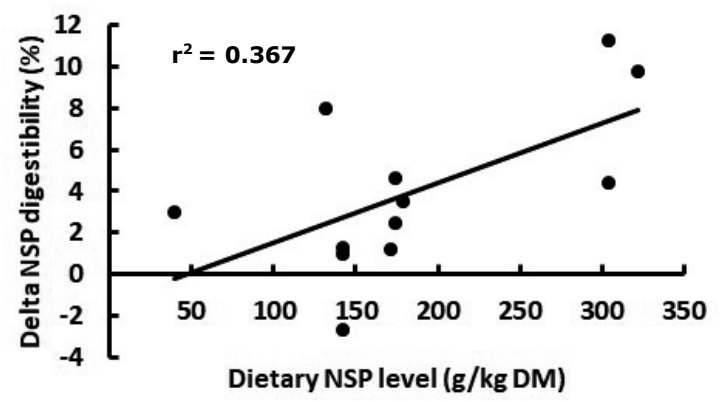

Figure 7 relationship between the dietary non-starch polysaccharide (NSP) level $(\mathrm{g} / \mathrm{kg}$ DM) and the $\Delta$ NSP digestibility (\%).

In this thesis, it is clearly demonstrated that NSP can be partly digested. However, which part of the NSP fraction is digested is unclear. When plant ingredients are used, the NSP composition will always contain a wide variety of NSP types (arabinoxylans, mixed-linked $\beta$-glucans, mannans, galactomannans, glucomannans, aribans, galactans and arabinogalactans, etc.) (Choct, 1997; Sinha et al., 2011). This makes it difficult to quantify the NSP digestibility of different ingredients and types of NSP. In other monogastric animals, like poultry and pigs, it is well established that fermentability varies considerably among different types of NSP; for example, lignin and cellulose are very resistant and pectins usually undergo complete fermentation (Williams et al., 2001, 2017). Likewise, the endogenous breakdown of different types of NSP may differ in tilapia, some NSP 
may be partly broken down while others are completely inert to digestion/fermentation. Measuring the constituent sugars of the NSP fraction in feed and faeces would allow the digestibility of the different NSP fractions and ingredients to be quantified. This information, would also enable NSP digestibility to be estimated in different diet formulations, and thus the digestible energy originating from NSP. Furthermore, when measuring NSP digestibility under enzyme supplementation, an indication of how successful the supplementation of enzymes is in increasing the availability of the targeted fraction can be obtained. Likewise, NSP digestibility might be measured to indicate which fraction is made available by phytase supplementation, or if phytase and xylanase compete for the same substrate etc.

\section{Probiotics}

In this thesis, probiotics were used in a single experiment (Chapter $\mathbf{7}$ and 8 ) with the main aim of improving diet quality. Probiotics improved the fat digestibility (Chapter 7). This may be related to the observed trend for a higher Ca availability in the proximal gut (Chapter 8), as Ca plays an important role in the digestion of lipids (Fave et al., 2004; Hu et al., 2010). Besides increasing fat digestibility, probiotics did not improve nutrient digestibility. Although, the effect of probiotics on nutrient digestibility was limited, probiotics did improve growth performance. Besides the increase in fat digestibility, fish fed the treatments with probiotics had a higher body ash content and increased levels of retained P. As demonstrated (Figure 5), increasing levels of available $P$ play an important role in enhancing growth. Although $\mathrm{P}$ availability is not affected by probiotic supplementation, a higher retention of $P$, and thus efficiency (although not significant), will have the same effect. In addition, the energy requirements for maintenance were reduced with probiotics, leaving more energy available for growth. The ability of probiotics to reduce the cost of maintenance have not previously been reported. The reduction in the energy cost of maintenance (Chapter 7 ) could be related to the various effects that probiotics can have on the gut environment (Hai, 2015; Dai et al., 2019; Dawood et al., 2019), which may lower the energy cost of digestion (part of maintenance). Although higher levels of volatile fatty acids (VFA) were expected with the use of enzymes (Chapter 8), through the breakdown of NSP, probiotics increased the total concentration of VFA in the proximal gut. How this increase in the proximal gut affected the microbiota remains unclear, as the microbial composition was only analysed in the distal gut. Although the probiotics did not break down NSP and phytate, the presumed positive effects of probiotics on gut health, as reported in the literature (Adeoye et al. 2016b; Dawood et al. 2019), could partly compensate for the negative effects of NSP and phytate, thus improving the tolerance of fish to these ANFs.

\section{Interactions between enzyme(s) and probiotics}

Synergy between xylanase and phytase was observed on the growth parameters, indicated by an interaction effect $(P<0.05)$ (Chapter 5). Hereby the effect of 
xylanase on growth was small, however when combined with phytase, xylanase enhanced growth. The observed synergy on growth was not explained by the digestibility, where no interactions occurred. However, for the $\mathrm{N}$ balance an interaction occurred, resulting in the highest $\mathrm{N}$ efficiency and retained $\mathrm{N}$ when both phytase and xylanase were supplemented. In general, the $\mathrm{N}$ efficiency is largely dependent on the dietary ratio between digestible protein ( $D P$, in $\mathrm{mg}$ ) and digestible energy ( $D E$, in $k J)$. In many studies in fish fed protein-rich diets, increasing the dietary non-protein energy led to better protein utilisation for growth, through protein sparing, i.e. less protein used to cover energy needs (Kim and Kaushik, 1992; Herme et al. 1993; Kroghdahl et al., 2005). With enzyme supplementation (Chapter 5), the digestible non-protein fraction increased, predominantly by improving the digestibility of NSP, thereby lowering the DP/DE ratio. The change in DP/DE was negatively related to $\mathrm{N}$ efficiency (Figure $8 \mathrm{a} ; \mathrm{r}^{2}=$ 0.893 ) and thus growth (Chapter 5). Likewise, changes in the DP/DE ratio, as a result of supplementation with enzymes and probiotics (Chapter 7), explained $97.1 \%$ of the variation in $\mathrm{N}$ efficiency. However, the DP/DE across studies (Chapter 5 and 7 ) did not explain the variation in $\mathrm{N}$ efficiency $(P>0.1)$. Besides changes in DP/DE, the enzymes and probiotics also changed the available $P$ level. Combining the data of both chapters revealed a strong positive correlation between the available $\mathrm{P}$ level ( $\mathrm{g} / \mathrm{kg} \mathrm{DM}$ diet) and the $\mathrm{N}$ efficiency (Figure $8 \mathrm{~b} ; P<0.01$ ). An increase in $\mathrm{N}$ efficiency can partly explain the observed effect of $\Delta$ available dietary $P$ on growth (Figure 5). A synergistic effect between enzymes was not observed (Chapter 4 and 6), which does not exclude a possible synergy between phytase and xylanase where they are only supplemented as a cocktail. To the best of our knowledge, interactions between enzymes have not been observed in fish before. The factor(s) underlying the increase in $\mathrm{N}$ efficiency and growth requires further investigation.
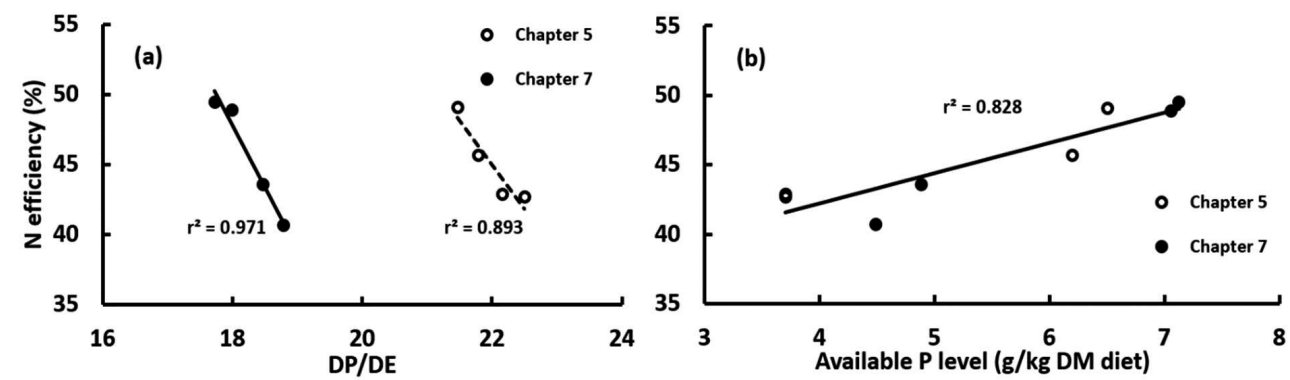

Figure 8 (a) relationship between DP/DE (ratio between digestible protein ( $D P$, in $\mathrm{mg}$ ) and digestible energy ( $D E$, in $k J)$ ) and the nitrogen $(N)$ efficiency (\%; retained N/digestible $N)$;

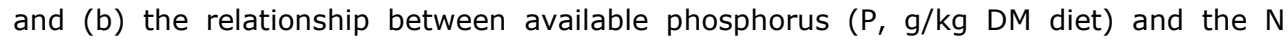
efficiency.

It was hypothesised that the combination of enzymes and probiotics in the diet could have a synergistic effect. This is by increasing the availability of substrates (through the use of enzymes) for the probiotics and by promoting the abundance 
of beneficial bacteria, while increasing NSP degradation. However, no synergy was observed between enzymes and probiotics (Chapter 7 and 8). Nevertheless, a few parameters do suggest a small complementary effect, resulting in, for example, the highest fat digestibility, $\mathrm{P}$ efficiency and lowest energy requirements for maintenance (Chapter 7). In addition, the bacterial species to species cooccurrence patterns and network complexity (Chapter 8), when enzymes and probiotics are combined, suggest a more stable gut microbiome environment. A more stable gut microbiome may contribute to the lower observed energy requirements for maintenance (Chapter $\mathbf{7}$ ). Although enzymes and probiotics did not show a positive interaction, their combination might still be beneficial, as both influence different mechanisms.

\section{Implication for other fish species}

Throughout the experiments the enzymes were effective in enhancing nutrient digestibility and growth in Nile tilapia. The effect of enzymes has been studied in multiple species, including; silver perch (Stone et al., 2003), rainbow trout (Ogunkoya et al., 2006; Dalgsgaard et al., 2012), Japanese seabass (Ai et al., 2007), African catfish (Yigit and Olmez, 2011) and great sturgeon (Ghnomi et al., 2012). In these studies, a variety of enzyme cocktails were used, which all included at least one type of NSP-degrading enzyme. There is some disagreement between these studies on the effect of supplementation with different enzymes, in some studies the enzymes improved the nutrient digestibility and/or growth, while in others they did not. The large variety of combinations of enzymes, species and diets used across studies, makes it hard to interpret what effects the enzymes may have. This thesis showed that with enzyme supplementation there was more to gain when the diet was of low quality (i.e. high levels of dietary phytate and NSP). How well fish can deal with high levels of plant ingredients and ANFs is partly related to the physiology and morphology of the GI-tract, which is linked to the natural feeding habit (omnivores vs. carnivorous). This will influence the maximum inclusion level of low-quality crop residues, and thus the level of ANFs, like NSP and phytate. Thereby the potential for improvement with exogenous enzymes is smaller for fish species that require high quality diets, like salmon and yellowtail kingfish. Besides the diet-related factors identified in this thesis (composition and level of ANF), the physiology of the fish is expected to play a major role in the response to enzyme supplementation.

This study suggests that supplemented enzymes were mainly active in the stomach of tilapia, where the $\mathrm{pH}$ is low (between 1 to 4.5 ) (Chapter 8) (Leenhouwers et al., 2007; Saravanan et al., 2013). Commercial phytase products are generally active in a pH range of 2.5-5.5 (Kumar et al., 2012; Dersjant-Li et al., 2015). Likewise, most exogenous NSP-degrading enzymes have a $\mathrm{pH}$ optimum between 4 and 5 (Svihus, 2010). Therefore, the optimal pH of the supplemented enzyme(s) and the $\mathrm{pH}$ along the GI-tract should match in order to be successful. For example, agastric fish (e.g. common carp) have a higher $\mathrm{pH}$ (6.2-7.7) along the whole GItract (Schäfer et al. 1995), so the enzymes as supplemented in this thesis are 
expected to be less effective. This does not mean enzymes cannot be used to improve diet quality for agastric fish. Enzymes can vary according to their source, enabling catalytic activity to occur in conditions of both low and high pH (Svihus, 2010). Moreover, the presence of other additives could influence the activity of the supplemented enzymes. Organic acids, such as formic and citric acids, are known to reduce the $\mathrm{pH}$ in the stomach and in the proximal intestine through delivery of $\mathrm{H}^{+}$(Lim et al., 2015; Dawood et al., 2018). Aligning the $\mathrm{pH}$ of the enzyme and the $\mathrm{pH}$ in the GI-tract should lead to an optimal efficiency. Furthermore, the $\mathrm{pH}$ in the GI-tract and the optimal pH of the enzyme might influence the optimal dose of the enzyme, thereby having an impact on the economic viability of the product (Adeola and Cowieson, 2011; Cowieson and Ravindran, 2008).

Like $\mathrm{pH}$, temperature has a large effect on enzyme activity. The temperature optimum is largely dependent on the source and type of enzyme. However, in general the optimum temperature for the enzyme(s) is well above that of the fish. For instance, for commercial phytase and xylanase, the optimum temperature is between $40-80{ }^{\circ} \mathrm{C}$ for phytase (Cao et al., 2007) and between $35-70{ }^{\circ} \mathrm{C}$ for xylanase (Lenartovicz et al., 2002; Lu et al., 2003; Ding et al., 2018), depending on the source. The (optimum) temperature for fish aquaculture is species dependent. Therefore, for warm water species, like tilapia and African catfish, the rearing temperature is closer to that of the enzyme's optimum temperature range, compared to cold-water species, like salmon and char.

Instead of supplementing enzymes to prepared diets, which are then activated in the fish, the raw ingredients can also be pre-treated with enzymes. Multiple studies, with enzymes such as phytase, have shown improved $\mathrm{P}$ availability and growth with pre-treated ingredients, like soybean meal (Cain and Garling, 1995; Storebakken et al., 1998; Cao et al., 2008). By pretreating ingredient(s), the P availability will be less affected by the physiology of the fish. However, pretreatment is more costly as additional production steps are required (soaking, drying, etc.). Enzyme supplementation was also successful in improving diet quality in the present study. This suggests that enzyme supplementation is potentially effective for a wide range of fish species, especially fish species sharing a similar biology. However, how environmental factors, like water temperature and salinity (osmoregulation), as well as fish physiology (i.e. gut length and pH GItract), potentially affect the enzyme response requires further elucidation.

\section{Implications for production systems}

All experiments were performed in flow-through glass tanks connected to a recirculating aquaculture system (RAS). In practice, tilapia is mostly cultured in ponds, cages or lakes. In flow-through and cage culture systems, it is important to minimise organic and inorganic waste production by the fish (faecal, branchial and urinary). This waste directly impacts the surrounding water body. Improving the efficiency of nutrient and energy utilisation for growth reduces the waste production, thus lowering the environmental impact of aquaculture. $P$ enrichment 
of natural waters is one of the main triggers of eutrophication. This has a negative impact on the aquatic ecosystem, by stimulating primary production and eventually leading to anoxic conditions (Mainstone and Parr, 2002; ChávezCrooker and Obreque-Contreras, 2010; Oliveira and Machado, 2013). In this thesis, there was a higher retention of $P$ when the diet was supplemented with enzymes, thus reducing the excretion of $P$ (faecal, branchial and urinary) into the environment by up to 38\% (Chapter 5). Furthermore, growth was enhanced, which also lowered the feed input required to maintain biomass production. The combined effect of higher $P$ retention and lower feed input (Chapter 5), lowered the $\mathrm{P}$ load (via water discharge) to the surrounding environment by $46 \%$.

In RAS, minerals can accumulate in the water (including $\mathrm{P}$ ), which can lead to waterborne mineral uptake by fish (Martins et al., 2010). Prabhu et al. (2017) examined the effect of different water exchange rates in RAS on the mineral requirements of common carp. They found that fish in low water exchange RAS (with denitrification) had higher whole body levels of minerals ( $\mathrm{P}, \mathrm{Ca}, \mathrm{Mg}, \mathrm{Na}, \mathrm{Ka}$, $\mathrm{Fe}, \mathrm{Mn}$ and Se; $\mathrm{g} / \mathrm{kg}$ fresh weight), compared to fish in high water exchange RAS. In this thesis, branchial and urinary (no distinction made) $\mathrm{P}$ losses occurred (Chapter $\mathbf{5}$ and $\mathbf{7}$ ). This is in contrast to the negative $\mathrm{P}$ values found by Schneider et al. (2004), which indicate $P$ uptake from the water. Whether or not fish take up $P$ (and other minerals) from the water is related to the amount of available minerals in the feed, as well as the concentration of available minerals in the water (Rodehutscord, 1996; Rodehutscord et al., 2000; Prabhu et al., 2017). When the $P$ availability in the feed is above the $P$ requirement to sustain maximal $P$ content, $P$ excretion will increase and there will be no need for water borne $P$ uptake. Generally, in RAS with low water exchange, a denitrification reactor is used in order to ensure good water quality, while minimising water exchange. In addition, denitrification is known to make largely insoluble minerals from faecal waste soluble (Goddek et al., 2016; Goddek et al., 2018). When denitrification occurs, phytate is broken down and $\mathrm{P}$ becomes soluble in water; this, in combination with low water exchange, can lead to accumulation of $P$ in the water. When available $P$ can be taken up from the water, there is less need for available $P$ from the diet.

For the culture of fish in ponds, Kabir et al. (2019) showed that more than $50 \%$ of tilapia growth could be attributed to a natural (food web-based) diet. Ekasari et al. (2015) found this to be a predominantly biofloc-based diet. Bioflocs are often defined as "aggregates of bacteria, algae, or protozoa, held together in a matrix, along with particulate organic matter" (El-Sayed 2019). The use of bioflocs, mainly as a water treatment technology for intensive aquaculture, is undergoing rapid growth. In ponds, the productivity of the natural food web, including bioflocs, is dependent on the $\mathrm{C} / \mathrm{N}$ ratio. Historically, external carbon sources are added to fish ponds in order to increase the $\mathrm{C} / \mathrm{N}$ ratio, which favours the growth of heterotrophic bacteria. Kabir et al. (2019) demonstrated that the $\mathrm{C} / \mathrm{N}$ ratio can also be altered by dietary composition, by lowering the dietary DP/DE ratios. This can be achieved by adding low-quality ingredients to fish feed, which stimulates the natural food web. Kabir et al. (2019) showed that a diet with a low DP/DE ratio, combined with 
a high NSP fraction, resulted in a higher faeces carbon content. Microbes in the water can use this additional carbon as an energy source to fix available N. As starch is highly digestible, most fecal carbon includes NSP. Improvement in carbohydrate and NSP digestibility, with exogenous enzyme supplementation, directly provides more energy to the fish. However, the subsequent reduction in faeces carbohydrate level may affect the natural food web and thus the formation of bioflocs. Therefore, further assessment of the impact of enzyme supplementation in pond culture feeds is required.

There is less known about the role of $P$ in pond food web dynamics. Levels of $P$ in bioflocs have been reported between $12.2-30 \mathrm{~g} / \mathrm{kg}$ dry weight (Kuhn et al., 2010; Rajkumar et al., 2016). This is higher than the P requirement $(4.0 \mathrm{~g} / \mathrm{kg})$ for tilapia, as stated by NRC (2011). Bioflocs are known to trap organic particles and solids (De Schryver et al., 2013; Ekasari et al., 2014) and thereby probably also phytate. It is suggested that certain microbes, which aggregate in bioflocs, can produce microbial phytase (Ekasari et al., 2019). Like the microorganisms in bioflocs, faeces containing phytate trapped in the sediment may be partially mineralised into phosphate, which can then potentially be fixed by bioflocs and taken up by the fish. Phytate, which is partially broken down by dietary phytase into IP5, IP4, etc., may potentially be more accessible to microorganisms. In addition, it is not clear if enzymes supplemented in the diet (i.e. xylanase and phytase) of fish can avoid protein hydrolysis in the GI-tract and be partially excreted in the faeces. If this is the case, the breakdown of phytate and NSP could continue (although slowly) outside of the fish, for example in the sediment. When fish can take up $P$, either from the water or from the natural food web (including bioflocs), the need for available $P$ from the diet decreases. Likewise, when phytate bound $P$ is made available through, for instance, denitrification or microorganisms in the pond or biofloc, the need for endogenous phytase may be redundant. Therefore, the requirement for $\mathrm{P}$ in the diet, and the need to supplement phytase in order to improve the $P$ availability, is expected to be dependent on the production system used (i.e. RAS, pond or cage culture). The impact and potential application of enzymes, in relation to the culture conditions (RAS, pond or cage culture), requires further elucidation.

\section{Future perspectives and contribution to circularity}

One of the main aims of this thesis was to understand how to enhance the digestibility of aquafeeds that contain increasing quantities of low quality plantbased ingredients, in line with the goals for circular food production. This thesis shows that enzymes, when supplemented to aquafeeds, enhance nutrient digestibility of low quality plant-based ingredients. Hereby it is shown that dietary supplementation of enzymes has a greater potential to improve digestibility and/or growth, when the diet is of low quality or when anti-nutritional effects are expressed (Chapter 3 and $\mathbf{4}$ ). It can be anticipated that the use of low quality ingredients will further increase with the rising demand for aquafeeds, thus reinforcing the convenience of exogenous enzyme supplementation. In addition, 
the proportion of low quality ingredients that can be included in aquafeeds may be limited by their level of ANFs (Francis et al., 2001). Reducing the negative effect of NSP and phytate, by improving their bioavailability, may also enable a positive shift in the proportion of low quality ingredients in aquafeeds. When the antinutritional effects of plant co- and by-products are partially neutralised, they may be incorporated at higher levels without adverse effects. Likewise, probiotics may partially reduce the negative effects of, for example, NSP on gut morphology, physiology and functioning. This could further contribute to the use of a greater quantity of low quality ingredients. However, this needs further investigation.

In the context of circularity, it is important to improve the bioavailability of phytate-bound $P$ from plant ingredients, in order to reduce pressure on the use of inorganic mined phosphorus. Phytase was shown to be successful in improving the availability of $\mathrm{P}$ from phytate, by up to $72 \%$ (Chapter 5 ). The amount of inorganic $\mathrm{P}$ could be completely replaced by $\mathrm{P}$ originating from phytate, as long as the requirements for $\mathrm{P}$ are met.

In general, improved feed efficiency through the use of enzymes and/or probiotics, reduces the amount of feed required, thus enhancing circularity. A higher feed efficiency, and thus a lower amount of feed, could also allow for higher inclusion levels of "low" quality feed ingredients, such as certain plant by- and co-products. This can all contribute to reducing the pressure on, and need for, crops as animal feed. With about $70 \%$ of agricultural land currently used for livestock (arable land and grassland), the expansion of animal production has been the main driver for converting forests, and other natural landscapes, into agricultural land (Van Zanten et al., 2018). Therefore, improving the feed efficiency of low quality diets can contribute to reducing the impact of aquaculture, on both land-use and global warming.

Whether supplements, like probiotics and enzymes, are used on a commercial scale largely depends on their economic viability. The extra cost of enzyme supplementation should be compensated for by the subsequent additional income generated. In chapter 3, an economic evaluation showed that the addition of enzymes was cost effective, as approximately $9 \%$ extra growth could be expected. In the studies in chapters 4, 5, 7 a similar, or greater, improvement in growth was achieved with enzyme(s) supplementation. Although the improvement in growth is worth the investment, it does not necessarily mean that these diets can compete with current commercial diets. There was an improvement in growth with enzyme supplementation (Chapter 3), but it should be noted that the reference diet (without enzymes) enabled better growth, when compared with the lower quality diets (with higher levels of NSP and phytate), even when they were supplemented with enzymes. The diet quality (choice of ingredients) is a tradeoff between quality and cost, therefore a cheaper low quality diet, which will benefit from enzyme supplementation, may be more cost effective compared to a high quality diet. Moreover, whether the use of enzymes is cost effective may also be dependent on the culture system (i.e. presence of natural food). 


\section{Conclusions}

This thesis assessed whether enzyme and probiotic supplementation can successfully improve the nutritional value of low quality diets. For Nile tilapia, the nutritional value of low quality diets can indeed be improved by these supplements, with enhanced growth performance as a result. This means that a greater proportion of low quality plant co- and by products could be incorporated into aquafeeds, which contributes to a circular food economy.

The main conclusions of the thesis, regarding Nile tilapia, are:

- NSP is not inert to digestion.

The meta-analysis conducted in this thesis, across studies and experiments, showed that tilapia partially digested NSP, which is linked to fermentation. The variation in NSP digestibility is large, with various factors (i.e. NSP composition) expected to influence NSP digestion.

- Phytase improved the availability of $\mathrm{P}, \mathrm{Ca}$ and crude ash fractions in the diet.

Besides improving the mineral availability, phytase was shown to improve the digestibility of DM, energy, carbohydrate, NSP, fat and crude protein. The effect of phytase supplementation on $\mathrm{P}$ and $\mathrm{Ca}$ availability was dependent on the phytase dose.

- Xylanase has the potential to improve nutrient digestibility.

The effect of xylanase on nutrient digestibility is less clear, compared to phytase. Xylanase was shown to improve the digestibility of DM, energy, carbohydrate, NSP, fat and crude protein in some studies. Xylanase had no effect on the mineral availability. The effect of xylanase was shown to be independent of the xylanase dose.

- $\beta$-glucanase did not improve nutrient digestibility.

In the only study where $\beta$-glucanase was supplemented, it did not affect the nutrient digestibility of any of the measured nutrients, or influence growth.

- Probiotic supplementation improved fat digestibility.

Probiotic supplementation had a large effect on fat digestibility, with a $\Delta$ fat ADC of $2.7 \%$ for the diet supplemented only with probiotics. Although probiotics improved fat digestibility, their effect on other nutrients was minor.

- Probiotics improved growth and reduced the energy requirements for maintenance.

The effect of probiotics on nutrient digestibility was minimal. It can therefore be concluded that, with probiotic supplementation, the observed reduction in energy 
requirements for maintenance is the main reason for the increase in growth. The effect of probiotics was only observed in the absence of enzymes.

- In Nile tilapia, the available $P$ requirement is $>7 \mathrm{~g} / \mathrm{kg}$ DM feed.

Supplementation with phytase, of diets formulated with levels of $P$ above the currently published requirement ( $4 \mathrm{~g} / \mathrm{kg}$; NRC, 2011), increased the amount of available dietary $\mathrm{P}$ and resulted in a large increase in growth and body $\mathrm{P}$ content.

- The combination of enzymes and probiotics enhanced microbial interactions in the distal gut.

Network analysis, based on the abundance patterns of different types of gut microbiota, showed that interactions between microbes became more complex when enzymes and probiotics were supplemented. This is potentially due to changes in substrate availability and $\mathrm{pH}$. Such results, together with an observed increase in abundance of beneficial gut microbes, such as Lactobacillus and Bacillus species, indicates higher microbiota stability. This has positive impacts for gut health.

- Enzymes, supplemented to the diet, are mainly active in the stomach.

Differences in P availability, with enzyme supplementation, appeared first in the proximal gastrointestinal tract (GI-tract) and this difference remained from there onwards, up until the distal GI-tract. This indicates that the enzymes were mainly active (breaking down phytate) in the stomach and also potentially in the proximal GI-tract.

- The effect of enzyme supplementation is dependent on diet quality.

It was observed that the increase in availability of $\mathrm{P}$ and $\mathrm{Ca}$ is strongly dependent on the $P$ availability in the control diet. A larger improvement is observed when the $\mathrm{P}$ availability in the control diet is low. A low $\mathrm{P}$ availability in the control diet can be linked to a lower diet quality (i.e. higher dietary levels of phytate and NSP). A similar trend, but smaller, is observed for fat, protein, carbohydrate and NSP digestibility.

- Synergy can occur between phytase and xylanase.

Phytase and xylanase had a synergistic effect on both growth and the $\mathrm{N}$ balance. Growth with the control diet (no enzymes) and with the xylanase diet were similar, whereas fish fed the diet with phytase showed improved growth. The effect of phytase on growth was further enhanced when xylanase was supplemented. A similar trend was observed for the $\mathrm{N}$ balance, with the highest $\mathrm{N}$ efficiency and $\mathrm{N}$ retention obtained when both enzymes were supplemented. This synergistic effect was not demonstrated across all studies, suggesting that it is context (diet) dependent. 



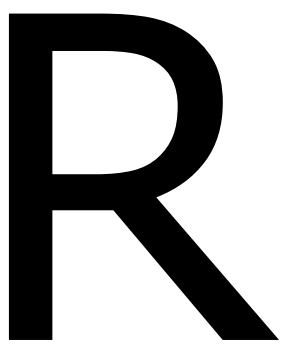

References 
A

AAFCO, 2000. Official Publication-Association of American Feed Control Officials. Atlanta.

Abarike, E.D., Cai J., Lu Y., Yu H., Chen L., Jian J., Tang J., Jun L., Kuebutornye F.K., 2018. Effects of a commercial probiotic BS containing Bacillus subtilis and Bacillus licheniformis on growth, immune response and disease resistance in Nile tilapia, Oreochromis niloticus. Fish Shellfish Immun. 82, 229-238.

Abdel-Latif, H.M.R., Abdel-Tawwab, M., Dawood, M.A.O., Menanteau-Ledouble, S., El-Matbouli, M., 2020. Benefits of Dietary Butyric Acid, Sodium Butyrate, and Their Protected Forms in Aquafeeds: A Review. Rev. Fish. Sci. Aquauc. 1-28.

Adeola, O., Cowieson, A., 2011. Board-invited review: opportunities and challenges in using exogenous enzymes to improve nonruminant animal production. J. Anim. Sci. 89, 3189-3218.

Adeoye, A.A, Jaramillo-Torres, A., Fox, S., Merrifield, D., Davies, S., 2016a. Supplementation of formulated diets for tilapia (Oreochromis niloticus) with selected exogenous enzymes: Overall performance and effects on intestinal histology and microbiota. Anim. Feed Sci. Technol. 215, 133-143.

Adeoye, A.A., Yomla, R., Jaramillo-Torres, A., Rodiles, A., Merrifield, D.L., Davies, S.J., 2016b. Combined effects of exogenous enzymes and probiotic on Nile tilapia (Oreochromis niloticus) growth, intestinal morphology and microbiome. Aquaculture 463, 61-70.

Ai, Q., Mai, K., Zhang, W., Xu, W., Tan, B., Zhang, C., Li, H., 2007. Effects of exogenous enzymes (phytase, non-starch polysaccharide enzyme) in diets on growth, feed utilization, nitrogen and phosphorus excretion of Japanese seabass, Lateolabrax japonicus. Comp. Biochem. Physiol. Part A Mol. Integr. Physiol. 147, 502-508.

Al-Deriny, S.H., Dawood, M.A.O., Zaid, A.A.A., El-Tras, W.F., Paray, B.A., Van Doan, H., Mohamed, R.A., 2020. The synergistic effects of Spirulina platensis and Bacillus amyloliquefaciens on the growth performance, intestinal histomorphology, and immune response of Nile tilapia (Oreochromis niloticus). Aquac. Rep. 17, 100390.

Alonso, R., Aguirre, A., Marzo, F., 2000. Effects of extrusion and traditional processing methods on antinutrients and in vitro digestibility of protein and starch in faba and kidney beans. Food Chem. $68,159-165$.

Aly, S.M., Ahmed, Y.A.G., Ghareeb, A.A.A., Mohamed, M.F., 2008a. Studies on Bacillus subtilis and Lactobacillus acidophilus, as potential probiotics, on the immune response and resistance of Tilapia nilotica (Oreochromis niloticus) to challenge infections. Fish Shellfish Immun. 25, 128-136.

Aly, S.M., Mohamed, M.F., John, G., 2008b. Effect of probiotics on the survival, growth and challenge infection in Tilapia nilotica (Oreochromis niloticus). Aquac. Res. 39, 647-656.

Amirkolaie, A.K., Leenhouwers, J.I., Verreth, J.A., Schrama, J.W., 2005. Type of dietary fibre (soluble versus insoluble) influences digestion, faeces characteristics and faecal waste production in Nile tilapia (Oreochromis niloticus L.). Aquac. Res. 36, 1157-1166.

Amirkolaie, A.K., Verreth, J.A., Schrama, J.W., 2006. Effect of gelatinization degree and inclusion level of dietary starch on the characteristics of digesta and faeces in Nile tilapia (Oreochromis niloticus (L.)). Aquaculture, 260, 194-205.

Amirkolaie, A.K., 2011. Reduction in the environmental impact of waste discharged by fish farms through feed and feeding. Rev. Aquacult. 3, 19-26.

Annison, G., 1993. The role of wheat non-starch polysaccharides in broiler nutrition. Crop and Pasture Sci. $44,405-422$.

Apata, D.F., 2008. Growth performance, nutrient digestibility and immune response of broiler chicks fed diets supplemented with a culture of Lactobacillus bulgaricus. J. Sci. Food Agric. 88, 1253-1258.

Argenzio, R., Southworth, M., Stevens, C., 1974. Sites of organic acid production and absorption in the equine gastrointestinal tract. Am. J. Physiol. 226, 1043-1050.

Ayyat, M., Labib, H.M., Mahmoud, H.K., 2014. A probiotic cocktail as a growth promoter in Nile Tilapia (Oreochromis niloticus). J. Appl. Aquaculture 26, 208-215.

Azevedo, P.A., Cho, C.Y., Leeson, S., Bureau, D.P., 1998. Effects of feeding level and water temperature on growth, nutrient and energy utilization and waste outputs of rainbow trout (Oncorhynchus mykiss). Aquat. Living Resour. 11, 227-238.

Azim, M., Verdegem, M.C.J., Mantingh, I., Van Dam, A., Beveridge, M., 2003. Ingestion and utilization of periphyton grown on artificial substrates by Nile tilapia, Oreochromis niloticus L. Aquac. Res. 34, 85-92.

B

Baeverfjord, G., Krogdahl, ̊.., 1996. Development and regression of soybean meal induced enteritis in Atlantic salmon, Salmo salar L., distal intestine: a comparison with the intestines of fasted fish. J. Fish Dis. 19, 375-387.

Balcázar, J.L., De Blas, I., Ruiz-Zarzuela, I., Vendrell, D., Calvo, A.C., Márquez, I., Gironés, O., Muzquiz, J.L., 2007. Changes in intestinal microbiota and humoral immune response following probiotic administration in brown trout (Salmo trutta). Br. J. Nutr. 97, 522-527.

Banerjee, S., Mukherjee A., Dutta D., Ghosh K., 2016. Non-starch polysaccharide degrading gut bacteria in Indian major carps and exotic carps. Jordan J. Biol. Sci. 147. 1-10.

Banerjee, G., Ray, A.K., 2017. The advancement of probiotics research and its application in fish farming industries. Res. Vet. Sci. 115, 66-77. 
Bedford, M., Schulze, H., 1998. Exogenous enzymes for pigs and poultry. Nutr. Res. Rev. 11, 91-114.

Bedford, M.R., 2000. Exogenous enzymes in monogastric nutrition-their current value and future benefits. Anim. Feed Sci. Tech. 86, 1-13.

Bedford, M. R., Cowieson, A. J., 2012. Exogenous enzymes and their effects on intestinal microbiology. Anim. Feed Sci. Technol. 173, 76-85.

Beg, Q., Kapoor, M., Mahajan, L., Hoondal, G., 2001. Microbial xylanases and their industrial applications: a review. Appl. Microbiol. Biotechnol. 56, 326-338.

Bergman, E., 1990. Energy contributions of volatile fatty acids from the gastrointestinal tract invarious species. Physiol. Rev. 70, 567-590.

Bendiksen, E.A., Johnsen, C.A., Olsen, H.J., Jobling, M., 2011. Sustainable aquafeeds: progress towards reduced reliance upon marine ingredients in diets for farmed Atlantic salmon (Salmo salar $L$.). Aquaculture 314, 132-139.

Bergman, E., 1990. Energy contributions of volatile fatty acids from the gastrointestinal tract in various species. Physiol. Rev. 70, 567-590.

Bergot, F., Breque J., 1983. Digestibility of starch by rainbow trout: effects of the physical state of starch and of the intake level. Aquaculture 34, 203-212.

Bugaut, M., 1987. Occurrence, absorption and metabolism of short chain fatty acids in the digestive tract of mammals. Comp. Biochem. Physiol. B Biochem. Mol. Biol. 86, 439-472.

Burel, C., Boujard, T., Kaushik, S.J., Boeuf, G., Van Der Geyten, S., Mol, K.A., Ribaillier, D., 2000. Potential of plant-protein sources as fish meal substitutes in diets for turbot (Psetta maxima): growth, nutrient utilisation and thyroid status. Aquaculture 188, 363-382.

\section{C}

Cain, K.D., Garling, D.L., 1995. Pretreatment of soybean meal with phytase for salmonid diets to reduce phosphorus concentrations in hatchery effluents. Prog. Fish-Cult. 57, 114-119.

Cao, L., Wang, W., Yang, C., Yang, Y., Diana, J., Yakupitiyage, A., Li, D., 2007. Application of microbial phytase in fish feed. Enzyme Microb. Tech. 40, 497-507.

Cao, L., Yang, Y., Wang, W.M., Yakupitiyage, A., Yuan, D.R., Diana, J.S., 2008. Effects of pretreatment with microbial phytase on phosphorous utilization and growth performance of Nile tilapia (Oreochromis niloticus). Aquac. Nutr. 14, 99-109.

Cao, G.T., Zeng, X.F., Chen, A.G., Zhou, L., Zhang, L., Xiao, Y.P., Yang, C.M., 2013. Effects of a probiotic, Enterococcus faecium, on growth performance, intestinal morphology, immune response, and cecal microflora in broiler chickens challenged with Escherichia coli K88. Poult. Sci. J. 92, 29492955.

Carter, C., Hauler, R., 2000. Fish meal replacement by plant meals in extruded feeds for Atlantic salmon, Salmo salar L. Aquaculture 185, 299-311.

Case, R.M., 1978. Synthesis, intracellular transport and discharge of exportable proteins in the pancreatic acinar cell and other cells. Biol. Rev. 53, 211-347.

Castillo, S., Gatlin III, D.M., 2015. Dietary supplementation of exogenous carbohydrase enzymes in fish nutrition: A review. Aquaculture 435, 286-292.

Centraal Veevoeder Bureau, 2016. CVB Veevoedertabel 2016. Wageningen: Federatie Nederlandse Diervoederketen 2016.

Chávez-Crooker, P., Obreque-Contreras, J., 2010. Bioremediation of aquaculture wastes. Curr. Opin. Biotechnol. 21, 313-317.

Chen, Y., Li, J., Xiao, P., Li, G., Yue, S., Huang, J., Zhu, W., Mo, Z., 2016. Isolation and characterization of $\mathrm{B}$ acillus spp. M 001 for potential application in turbot ( $S$ cophthalmus maximus L.) against $\mathrm{V}$ ibrio anguillarum. Aquac. Nutr. 22, 374-381.

Cheng, Z.J., Hardy, R.W., 2002. Apparent digestibility coefficients and nutritional value of cottonseed meal for rainbow trout (Oncorhynchus mykiss). Aquaculture 212, 361-372.

Cheng, Z.J., Hardy, R.W., 2003. Effects of extrusion processing of feed ingredients on apparent digestibility coefficients of nutrients for rainbow trout (Oncorhynchus mykiss). Aquac. Nutr. 9, 77-83.

Cheng, Z.J., Hardy, R.W., Verlhac, V., Gabaudan, J., 2004. Effects of microbial phytase supplementation and dosage on apparent digestibility coefficients of nutrients and dry matter in soybean productbased diets for rainbow trout Oncorhynchus mykiss. J. World Aquac. Soc. 35, 1-15.

Choct, M., Annison, G., 1992. The inhibition of nutrient digestion by wheat pentosans. Br. J. Nutr. 67, 123132.

Choct, M., 1997. Feed non-starch polysaccharides: chemical structures and nutritional significance. Feed Milling International 191, 13-26.

Choct, M., Kocher, A., 2000. Non-starch carbohydrates: Digestion and its secondary effects in monogastrics. Proc. Nutr. Soc. Aust. 24, 31-38.

Choct, M., 2006. Enzymes for the feed industry: past, present and future. World Poultry Sci. J. 62, 5-16.

Chou, R., Her, B., Su, M., Hwang, G., Wu, Y., Chen, H., 2004. Substituting fish meal with soybean meal in diets of juvenile cobia Rachycentron canadum. Aquaculture 229, 325-333.

Clements, K., Gleeson V., Slaytor, M., 1994. Short-chain fatty acid metabolism in temperate marine herbivorous fish. J. Comp. Physiol. B 164, 372-377.

Clements, K., Choat, J.H., 1995. Fermentation in tropical marine herbivorous fishes. Physiol. Zool. 68, 355-378. 
Clements, K.D., Angert, E.R., Montgomery, W.L., Choat, J.H., 2014. Intestinal microbiota in fishes: what's known and what's not. Mol. Ecol. 23, 1891-1898.

Collinder, E., Björnhag, G., Cardona, M., Norin, E., Rehbinder, C., Midtvedt, T., 2009. Gastrointestinal Host-Microbial Interactions in Mammals and Fish: Comparative Studies in Man, Mice, Rats, Pigs, Horses, Cows, Elks, Reindeers, Salmon and Cod. Microb. Ecol. Health Dis. 15, 66-78.

Collins, T., Gerday, C., Feller, G., 2005. Xylanases, xylanase families and extremophilic xylanases. FEMS microbiol. Rev. 29, 3-23.

Cooper, C.A., Whittamore, J.M., Wilson, R.W. (2010). Ca ${ }^{2+}$ driven intestinal $\mathrm{HCO}_{3}$ secretion and $\mathrm{CaCO}_{3}$ precipitation in the European flounder in vivo: influences on acid-base regulation and blood gas transport. Am. J. Physiol. Regul. Integr. Comp. Physiol. 298, 870-876.

Cowieson, A., Bedford, M., 2009. The effect of phytase and carbohydrase on ileal amino acid digestibility in monogastric diets: complimentary mode of action? World Poultry Sci. J. 65, 609-624.

Cowieson, A.J., Ravindran, V., 2008. Effect of exogenous enzymes in maize-based diets varying in nutrient density for young broilers: growth performance and digestibility of energy, minerals and amino acids. Br. Poult. Sci. 49, 37-44.

D

Dai, B., Hou, Y., Hou, Y., Qian, L., 2019. Effects of multienzyme complex and probiotic supplementation on the growth performance, digestive enzyme activity and gut microorganisms composition of snakehead (Channa argus). Aquac. Nutr. 25, 15-25.

Dalmo, R.A., Bøgwald J., 2008. B-glucans as conductors of immune symphonies. Fish Shellfish Immunol. 25, 384-396.

Dalsgaard, J., Verlhac, V., Hjermitslev, N.H., Ekmann, K.S., Fischer, M., Klausen, M., Pedersen, P.B., 2012. Effects of exogenous enzymes on apparent nutrient digestibility in rainbow trout (Oncorhynchus mykiss) fed diets with high inclusion of plant-based protein. Anim. Feed Sci. Technol. 171, 181191.

Dawood, M.A., Koshio, S., Esteban, M.Á., 2018. Beneficial roles of feed additives as immunostimulants in aquaculture: a review. Rev. Aquac. 10, 950-974.

Dawood, M.A., Koshio, S., Abdel-Daim, M.M., Van Doan, H., 2019. Probiotic application for sustainable aquaculture. Rev. Aquac. 11, 907-924.

Deb, P., Talukdar, S.A., Mohsina, K., Sarker, P.K., Sayem, S.M.A., 2013. Production and partial characterization of extracellular amylase enzyme from Bacillus amyloliquefaciens P-001. SpringerPlus 2, 154.

Degani, G., Viola, S., Yehuda, Y., 1997. Apparent digestibility of protein and carbohydrate in feed ingredients for adult tilapia (Oreochromis aureus $x$ Oreochromis niloticus). Isr. J. Aquac. 49, 115123.

Debnath, D., Sahu, N.P., Pal, A.K., Jain, K.K., Yengkokpam, S., Mukherjee, S.C., 2005. Mineral status of Pangasius pangasius (Hamilton) fingerlings in relation to supplemental phytase: absorption, wholebody and bone mineral content. Aquac. Res. 36, 326-335.

Deng, J., Chen, L., Mai, K., Mi, H., Zhang, L., 2016. Effects of replacing soybean meal with rubber seed meal on digestive enzyme activity, nutrient digestibility and retention in tilapia (Oreochromis niloticus $\times$ Oreochromis aureus). Aquac. Res. 48, 1767-1777

Dersjant-Li, Y., Awati, A., Schulze, H., Partridge, G., 2015. Phytase in non-ruminant animal nutrition: a critical review on phytase activities in the gastrointestinal tract and influencing factors. J. Sci. Food Agric. 95, 878-896.

Ding, C., Li, M., Hu, Y., 2018. High-activity production of xylanase by Pichia stipitis: purification, characterization, kinetic evaluation and xylooligosaccharides production. Int. J. Biol. Macromol. $117,72-77$.

Diógenes, A.F., Castro, C., Carvalho, M., Magalhães, R., Estevão-Rodrigues, T.T., Serra, C.R., Oliva-Teles, A., Peres, H., 2018. Exogenous enzymes supplementation enhances diet digestibility and digestive function and affects intestinal microbiota of turbot (Scophthalmus maximus) juveniles fed distillers' dried grains with solubles (DDGS) based diets. Aquaculture 486, 42-50.

Dhingra, D., Michael M., Rajput, H., Patil, R., 2012. Dietary fibre in foods: a review. J. Food Sci. Technol. $49,255-266$.

Dong, X.H., Guo, Y.X., Ye, J.D., Song, W.D., Huang, X.H., Wang, H., 2010. Apparent digestibility of selected feed ingredients in diets for juvenile hybrid tilapia, Oreochromis niloticus $\times$ Oreochromis aureus. Aquac. Res. 41, 1356-1364.

Dornez, E., Gebruers, K., Delcour, J.A., Courtin, C.M., 2009. Grain-associated xylanases: occurrence, variability, and implications for cereal processing. Trends Food Sci. Technol. 20, 495-510.

\section{E}

Ebihara, K., Schneeman, B.O., 1989. Interaction of bile acids, phospholipids, cholesterol and triglyceride with dietary fibers in the small intestine of rats. J. Nutr. 119, 1100-1106.

Ebeling, M.E., 1968. The Dumas method for nitrogen in feeds. J. AOAC Int. 51, 766-770. 
Ekasari, J., Angela, D., Waluyo, S. H., Bachtiar, T., Surawidjaja, E. H., Bossier, P., De Schryver, P., 2014. The size of biofloc determines the nutritional composition and the nitrogen recovery by aquaculture animals. Aquaculture 426, 105-111.

Ekasari, J., Rivandi, D.R., Firdausi, A.P., Surawidjaja, E.H., Zairin Jr, M., Bossier, P., De Schryver, P., 2015. Biofloc technology positively affects Nile tilapia (Oreochromis niloticus) larvae performance. Aquaculture 441, 72-77.

Ekasari, J., Suprayudi, M.A., Elas, P., Senja, R.K., 2019. The digestibility of biofloc meal from African catfish culture medium as a feed raw material for Pacific white shrimp. Jurnal Akuakultur Indonesia 18, 1-8.

El-Haroun, E., Goda, A.S., Chowdhury, K., 2006. Effect of dietary probiotic Biogen ${ }^{\circledR}$ supplementation as a growth promoter on growth performance and feed utilization of Nile tilapia Oreochromis niloticus (L.). Aquac. Res. 37, 1473-1480.

El-Saidy, D.M., Gaber, M., 2003. Replacement of fish meal with a mixture of different plant protein sources in juvenile Nile tilapia, Oreochromis niloticus (L.) diets. Aquac. Res. 34, 1119-1127.

El-Sayed, A.F.M., 2019. Tilapia culture. Academic Press. Chapter 13.10.3 Biofloc technology

El-Shafai, S.A., El-Gohary, F.A., Verreth, J.A., Schrama, J.W., Gijzen, H.J., 2004. Apparent digestibility coefficient of duckweed (Lemna minor), fresh and dry for Nile tilapia (Oreochromis niloticus L.). Aquac. Res. 35, 574-586.

Encarnação, P., 2016. Functional feed additives in aquaculture feeds. In Aquafeed formulation (pp. 217237). Academic Press.

Engelen, A.J., van der Heeft, F.C., Randsdorp, P.H., Somers, W.A., 2001. Determination of phytase activity in feed by a colorimentric enztmatic method: Collaboratory study. J. AOAC Int. 84, 629-633.

Engelhardt, von, W., Rönnau, K., Rechkemmer, G., Sakata, T., 1989. Absorption of short-chain fatty acids and their role in the hindgut of monogastric animals. Anim. Feed Sci. Technol. 23, 43-53.

Englyst, H., 1989. Classification and measurement of plant polysaccharides. Anim. Feed Sci. Technol. 23, 27-42.

$\mathbf{F}$

Falco, A., Miest, J.J., Pionnier, N., Pietretti, D., Forlenza, M., Wiegertjes, G.F., Hoole, D., 2014. $\beta$ - Glucansupplemented diets increase poly (I: C)-induced gene expression of Mx, possibly via Tlr3-mediated recognition mechanism in common carp (Cyprinus carpio). Fish Shellfish Immunol. 36, 494-502.

FAO, 2001. The composition of fish. Document prepared by J. Murray and J.R. Burt. No. 38.

FAO, 2018. The State of World Fisheries and Aquaculture. FAO of the United Nations, Rome.

FAO, 2020. The State of World Fisheries and Aquaculture 2020. Sustainability in Action, FAO Rome, Italy.

Farhangi, M., Carter, C.G., 2007. Effect of enzyme supplementation to dehulled lupin-based diets on growth, feed efficiency, nutrient digestibility and carcass composition of rainbow trout, Oncorhynchus mykiss (Walbaum). Aquac. Res. 38, 1274-1282.

Farm gate (2018, March 18). Chinese tilapia raw material prices higher, but set to drop again. Undercurrent News. Retrieved December 3, 2018, from: https://www.undercurrentnews.com/prices/\#/guangdong_tilapia_300_500

Farhat-Khemakhem, A., Blibech, M., Boukhris, I., Makni, M., Chouayekh, H., 2018. Assessment of the potential of the multi-enzyme producer Bacillus amyloliquefaciens US573 as alternative feed additive. J. Sci. Food Agric. 98, 1208-1215.

Faust, K., Raes, J., 2016. CoNet app: inference of biological association networks using Cytoscape. F1000Res. 5, 1519.

Fave, G., Coste, T., Armand, M., 2004. Physicochemical properties of lipids: new strategies to manage fatty acid bioavailability. Cell. Mol. Biol. 50, 815-832.

Fidopiastis, P.M., Bezdek, D.J., Horn, M.H., Kandel, J.S., 2006. Characterizing the resident, fermentative microbial consortium in the hindgut of the temperate-zone herbivorous fish, Hermosilla azurea (Teleostei: Kyphosidae). Mar. Biol. 148, 631-642.

Finegold, S.M., Vaisanen, M.-L., Molitoris, D.R., Tomzynski, T.J., Song, Y., Liu, C., Collins, M.D., Lawson, P.A., 2003. Cetobacterium somerae sp. nov. from Human Feces and Emended Description of the Genus Cetobacterium. Syst. Appl. Microbiol. 26, 177-181.

Fontagné, S., Silva, N., Bazin, D., Ramos, A., Aguirre, P., Surget, A., Power, D.M., 2009. Effects of dietary phosphorus and calcium level on growth and skeletal development in rainbow trout (Oncorhynchus mykiss) fry. Aquaculture 297, 141-150.

Forrest, I., Wainwright, T., 1977. The mode of binding of $\beta$-glucans and pentosans in barley endosperm cell walls. J. I. Brewing 83, 279-286.

Forster, I., Higgs, D., Dosanjh, B., Rowshandeli, M., Parr, J., 1999. Potential for dietary phytase to improve the nutritive value of canola protein concentrate and decrease phosphorus output in rainbow trout (Oncorhynchus mykiss) held in $11^{\circ} \mathrm{C}$ fresh water. Aquaculture 179, 109-125.

Francis, G., Makkar, H.P., Becker, K., 2001. Antinutritional factors present in plant-derived alternate fish feed ingredients and their effects in fish. Aquaculture 199, 197-227.

Fry, J.P., Love, D.C., MacDonald, G.K., West, P.C., Engstrom, P.M., Nachman, K.E., Lawrence, R.S., 2016. Environmental health impacts of feeding crops to farmed fish. Environ. Int. 91, 201-214. 
G

Gaber, M.M., 2005. The Effect of Different Levels of Krill Meal Supplementation of Soybean-based Diets on Feed Intake, Digestibility, and Chemical Composition of Juvenile Nile Tilapia Oreochromis niloticus, L. J. World Aquacult. Soc. 36, 346-353.

Gaber, M.M., 2006. The Effects of Plant-protein-based Diets Supplemented with Yucca on Growth, Digestibility, and Chemical Composition of Nile Tilapia (Oreochromis niloticus, L) Fingerlings. J. World Aquacult. Soc. 37, 74-81.

Garcia-Pérez, A., Alston, D.E., Cortés-Maldonado, R., 2000. Growth, survival, yield, and size distributions of freshwater prawn Macrobrachium rosenbergii and tilapia Oreochromis niloticus in polyculture and monoculture systems in Puerto Rico. J. World Aquacult. Soc. 31, 446-451.

Gatlin III, D.M., Barrows, F.T., Brown, P., Dabrowski, K., Gaylord, T.G., Hardy, R., Herman, E., Hu, G., Krogdahl, Å., Nelson, R., Overturf, K., Rust, M., Sealey, W., Skonberg, D., Souza, E.J., Stone, D., Wilson, R., Wurtele, E., 2007. Expanding the utilization of sustainable plant products in aquafeeds: a review. Aquac. Res. 38, 551-579.

Gerritsen, J., Hornung, B., Ritari, J., Paulin, L., Rijkers, G.T., Schaap, P.J., de Vos, W.M., Smidt, H., 2019. A comparative and functional genomics analysis of the genus (Romboutsia) provides insight into adaptation to an intestinal lifestyle. bioRxiv 845511.

German, D.P., Bittong, R.A., 2009. Digestive enzyme activities and gastrointestinal fermentation in woodeating catfishes. J. Comp. Physiol. B 179, 1025-1042.

Ghomi, M.R., Shahriari, R., Langroudi, H.F., Nikoo, M., von Elert, E., 2012. Effects of exogenous dietary enzyme on growth, body composition, and fatty acid profiles of cultured great sturgeon Huso huso fingerlings. Aquac. Int. 20, 249-254.

Giang, H.H., Viet, T.Q., Ogle, B., Lindberg, J.E., 2011. Effects of supplementation of probiotics on the performance, nutrient digestibility and faecal microflora in growing-finishing pigs. Asian-Australas. J. Anim. Sci. 24, 655-661.

Giatsis, C., Sipkema, D., Ramiro-Garcia, J., Bacanu, G.M., Abernathy, J., Verreth, J., Smidt, H., Verdegem, M.C.J., 2016. Probiotic legacy effects on gut microbial assembly in tilapia larvae. Sci. Rep. 6, 33965.

Goda, A.M.A., Mabrouk, H.A.-H.H., Wafa, M.A.E.-H., El-Afifi, T.M., 2012. Effect of using baker's yeast and exogenous digestive enzymes as growth promoters on growth, feed utilization and hematological indices of Nile tilapia, Oreochromis niloticus fingerlings. J. Agric. Sci. Technol. B. 2.

Goddek, S., Delaide, B.P., Joyce, A., Wuertz, S., Jijakli, M.H., Gross, A., Morgenstern, R., 2018. Nutrient mineralization and organic matter reduction performance of RAS-based sludge in sequential UASBEGSB reactors. Aquac. Eng. 83, 10-19.

Goddek, S., Espinal, C.A., Delaide, B., Jijakli, M.H., Schmautz, Z., Wuertz, S., Keesman, K.J., 2016. Navigating towards decoupled aquaponic systems: A system dynamics design approach. Water 8 , 303.

Gómez, G.D., Balcázar, J.L., 2008. A review on the interactions between gut microbiota and innate immunity of fish. FEMS Immunol Med. Microbiol. 52, 145-54.

Govoni, J.J, Boehlert, G.W., Watanabe, Y., 1986. The physiology of digestion in fish larvae. Environ. Biol. Fishes 16, 59-77.

Gupta, S., Lokesh, J., Abdelhafiz, Y., Siriyappagouder, P., Pierre, R., Sorensen, M., Fernandes, J.M.O., Kiron, V., 2019. Macroalga-Derived Alginate Oligosaccharide Alters Intestinal Bacteria of Atlantic Salmon. Front Microbiol. 10, 2037.

\section{$\mathbf{H}$}

Hagey, L.R., Møller, P.R., Hofmann, A.F., Krasowski, M.D., 2010. Diversity of Bile Salts in Fish and Amphibians: Evolution of a Complex Biochemical Pathway. Physiol. Biochem. Zool. 83, 308-321.

Hai, N.V., 2015. Research findings from the use of probiotics in tilapia aquaculture: A review. Fish Shellfish Immunol. 45, 592-597.

Haidar, M.N., Petie, M., Heinsbroek, L.T.N., Verreth, J.A.J., Schrama, J.W., 2016. The effect of type of carbohydrate (starch vs. nonstarch polysaccharides) on nutrients digestibility, energy retention and maintenance requirements in Nile tilapia. Aquaculture 463, 241-247.

Hamza, A., Fdhila, K., Zouiten, D., Masmoudi, A.S., 2016. Virgibacillus proomii and Bacillus mojavensis as probiotics in sea bass (Dicentrarchus labrax) larvae: effects on growth performance and digestive enzyme activities. Fish Physiol. Biochem. 42, 495-507.

Hansen, A.-C., Rosenlund, G., Karlsen, Ø., Koppe, W., Hemre, G.-I., 2007. Total replacement of fish meal with plant proteins in diets for Atlantic cod (Gadus morhua L.) I-Effects on growth and protein retention. Aquaculture 272, 599-611.

Hardy, R.W., Mugrditchian, D.S., Iwaoka, W.T., 1983. Storage stability of lipids in a dry salmonid diet. Aquaculture 34, 239-246.

Hardy, R.W, Fairgrieve, W., Scott, T., 1993. Periodic feeding of low-phosphorus diet and phosphorus retention in rainbow trout (Oncorhynchus mykiss). Colloques de I'INRA (France).

Hardy, R.W., 2010. Utilization of plant proteins in fish diets: effects of global demand and supplies of fishmeal. Aquac. Res. 41, 770-776. 
Hassaan, M.S., Mohammady, E.Y., Soaudy, M.R., Abdel Rahman, A.A., 2019. Exogenous xylanase improves growth, protein digestibility and digestive enzymes activities in Nile tilapia, Oreochromis niloticus, fed different ratios of fish meal to sunflower meal. Aquac. Nutr. 25, 841-853.

Hauville, M.R., Zambonino-Infante, J.L., Gordon Bell, J., Migaud, H., Main, K.L., 2016. Effects of a mix of Bacillus sp. as a potential probiotic for Florida pompano, common snook and red drum larvae performances and digestive enzyme activities. Aquac. Nutr. 22, 51-60.

Hemre, G.I, Lie, Ø., Sundby, A., 1993. Dietary carbohydrate utilization in cod (Gadus morhua): metabolic responses to feeding and fasting. Fish Physiol. Biochem. 10, 455-463.

Hemre, G.I., Mommsen, T., Krogdahl, \&., 2002. Carbohydrates in fish nutrition: effects on growth, glucose metabolism and hepatic enzymes. Aquac. Nutr. 8, 175-194.

Henken, A., Kleingeld, D., Tijssen, P., 1985. The effect of feeding level on apparent digestibility of dietary dry matter, crude protein and gross energy in the African catfish Clarias gariepinus (Burchell, 1822). Aquaculture 51, 1-11.

Heuze, V., Tran, G., Kaushik, S., 2020. Soybean meal. Feedipedia, a programme by INRA, CIRAD, AFZ and FAO, last updated on march 4. 2020.

Hlophe-Ginindza, S.N., Moyo, N.A., Ngambi, J.W., Ncube, I., 2016. The effect of exogenous enzyme supplementation on growth performance and digestive enzyme activities in Oreochromis mossambicus fed kikuyu-based diets. Aquac. Res. 47, 3777-3787.

Hoseinifar, S.H., Dadar, M., Ringø, E., 2017. Modulation of nutrient digestibility and digestive enzyme activities in aquatic animals: the functional feed additives scenario. Aquac. Res. 48, 3987-4000.

Hossain, M.M., Begum, M., Kim, I.H., 2015. Effect of Bacillus subtilis, Clostridium butyricum and Lactobacillus acidophilus endospores on growth performance, nutrient digestibility, meat quality, relative organ weight, microbial shedding and excreta noxious gas emission in broilers. Vet. Med. $60,77-86$.

Hu, M., Li, Y., Decker, E.A., McClements, D.J., 2010. Role of calcium and calcium-binding agents on the lipase digestibility of emulsified lipids using an in vitro digestion model. Food Hydrocoll. 24, 719725.

Hu, J., Ran, C., He, S., Cao, Y., Yao, B., Ye, Y., Zhou, Z., 2016. Dietary microbial phytase exerts mixed effects on the gut health of tilapia: a possible reason for the null effect on growth promotion. $\mathrm{Br}$. J. Nutr. 115, 1958-1966.

Hussain, S., Afzal, M., Rana, S.A., Javid, A., Iqbal, M., 2011. Effect of Phytase Supplementation on Growth Performance and Nutrient Digestibility of Labeo rohita Fingerlings Fed on Corn Gluten Meal-based Diets. Int. J. Agric. Biol. 13(6).

\section{I}

Ige, B.A., 2013. Probiotics use in intensive fish farming. Afr. J. Microbiol. Res. 7, 2701-2711.

ISO (International Organization for Standardization), 1978. Animal Feeding Stuffs - Determination of Crude Ash (ISO 5984).

ISO (International Organization for Standardization), 1997. Animal feeding stuffs - Determination of nitrogen content and calculation of crude protein content. Kjeldahl method (ISO 5983).

ISO (International Organization for Standardization), 1999. Animal Feeding Stuffs - Determination of Fat Content (ISO 6492).

\section{J}

Jiang, T.T., Feng, L., Liu, Y., Jiang, W.D., Jiang, J., Li, S.H., Zhou, X.Q., 2014. Effects of exogenous xylanase supplementation in plant protein-enriched diets on growth performance, intestinal enzyme activities and microflora of juvenile Jian carp (Cyprinus carpio var. Jian). Aquac. Nutr. 20, $632-645$.

Jiang, Y., Wang, Y., Zhang, Z., Liao, M., Li, B., Rong, X., Chen, G., 2019. Responses of microbial community structure in turbot (Scophthalmus maximus) larval intestine to the regulation of probiotic introduced through live feed. PLoS One 14, e0216590.

Jin, Y.L., Zhou, X.Q., Jiang, W.D., Wu, P., Liu, Y., Jiang, J., Feng, L., 2020. Xylanase supplementation in plant protein-enriched diets improves growth performance by optimizing the intestinal microflora and enhancing the intestinal immune function in grass carp grow-out (Ctenopharyngodon idella). Aquac. Nutr. 26, 781-800.

JøRgensen, H., Zhao, X.-Q., Knudsen, K.E.B., Eggum, B.O., 1996. The influence of dietary fibre source and level on the development of the gastrointestinal tract, digestibility and energy metabolism in broiler chickens. Br. J. Nutr. 75, 379-395.

Juanpere, J., Pérez-Vendrell, A.M., Angulo, E., Brufau, J., 2005. Assessment of potential interactions between phytase and glycosidase enzyme supplementation on nutrient digestibility in broilers. Poult. Sci. 84, 571-580.

Jung, J., Lee, M.G., 2014. Role of calcium signaling in epithelial bicarbonate secretion. Cell Calcium 55, 376-384.

Jutfelt, F., 2011. Barrier function of the gut. Encycl. Fish Physiol. From Genome to Environ. 2, $1322-1331$. 
$\mathbf{K}$

Kabir, K.A., Schrama, J.W., Verreth, J.A.J., Phillips, M.J., Verdegem, M.C J., 2019. Effect of dietary protein to energy ratio on performance of nile tilapia and food web enhancement in semi-intensive pond aquaculture. Aquaculture 499, 235-242.

Kaushik, S.J., de Oliva Teles, A., 1985. Effect of digestible energy on nitrogen and energy balance in rainbow trout. Aquaculture, 50, 89-101.

Kies, A., van Hemert, K., Sauer, W., 2001. Effect of phytase on protein and amino acid digestibility and energy utilisation. Worlds Poult. Sci. J. 57, 109-126.

Kihara, M., Sakata, T., 2002. Production of short-chain fatty acids and gas from various oligosaccharides by gut microbes of carp (Cyprinus carpio L.) in micro-scale batch culture. Comp. Biochem. Physiol. Part A Mol. Integr. Physiol. 132, 333-340.

Kim, J., Kaushik, S.J., 1992. Contribution of digestible energy from carbohydrates and estimation of protein/energy requirements for growth of rainbow trout (Oncorhynchus mykiss). Aquaculture $106,161-169$.

Knudsen, K.E.B., Jensen, B.B., Hansen, I., 1993. Digestion of polysaccharides and other major components in the small and large intestine of pigs fed on diets consisting of oat fractions rich in $\beta$ - D-glucan. Br. J. Nutr. 70, 537-556.

Knudsen, K.E.B., 1997. Carbohydrate and lignin contents of plant materials used in animal feeding. Anim. Feed Sci. Technol. 67, 319-338.

Konieczka, P., Nowicka, K., Madar, M., Taciak, M., Smulikowska, S., 2018. Effects of pea extrusion and enzyme and probiotic supplementation on performance, microbiota activity and biofilm formation in the broiler gastrointestinal tract. Br. Poult. Sci. 59, 654-662.

Köprücü, K., Özdemir Y., 2005. Apparent digestibility of selected feed ingredients for Nile tilapia (Oreochromis niloticus). Aquaculture 250, 308-316.

Krogdahl, A., Herme, G.I., Mommsen, T., 2005. Carbohydrates in fish nutrition: digestion and absorption in postlarval stages. Aquac. Nutr. 11, 103-122.

Kuebutornye, F.K.A., Abarike, E.D., Lu, Y. 2019. A review on the application of Bacillus as probiotics in aquaculture. Fish Shellfish Immunol. 87, 820-828.

Kuebutornye, F.K.A., Lu, Y., Abarike, E.D., Wang, Z., Li, Y., Sakyi, M.E., 2020. In vitro Assessment of the Probiotic Characteristics of Three Bacillus Species from the Gut of Nile Tilapia, Oreochromis niloticus. Probiotics Antimicrob. Proteins 12, 412-424.

Kuebutornye, F.K.A, Abarike, E.D., Sakyi, M.E., Lu, Y., Wang, Z., 2020. Modulation of nutrient utilization, growth, and immunity of Nile tilapia, Oreochromis niloticus: the role of probiotics. Aquac. Int. 28, 277-291.

Kuhn, D.D., Lawrence, A.L., Boardman, G.D., Patnaik, S., Marsh, L., Flick Jr, G.J., 2010. Evaluation of two types of bioflocs derived from biological treatment of fish effluent as feed ingredients for Pacific white shrimp, Litopenaeus vannamei. Aquaculture 303, 28-33.

Kumar, V., Sinha, A., Makkar, H., De Boeck, G., Becker, K., 2012. Phytate and phytase in fish nutrition. J. Anim. Physiol. Anim. Nutr. 96, 335-364.

Kumar, N., Sharma, R., Tripathi, G., Kumar, K., Dalvi, R.S., Krishna, G., 2016. Cellular metabolic, stress, and histological response on exposure to acute toxicity of endosulfan in tilapia (Oreochromis mossambicus). Environ. Toxicol. 31, 106-115.

Kuz'mina V.V., 1996. Influence of age on digestive enzyme activity in some freshwater teleosts. Aquaculture 148, 25-37.

$\mathbf{L}$

Lall, S.P., Hardy, R.W., 2003. 5 - The Minerals A2 - Halver, John E, Fish Nutrition (Third Edition). Academic Press, San Diego, pp. 259-308.

Lall, S.P., Lewis-McCrea, L.M., 2007. Role of nutrients in skeletal metabolism and pathology in fish-an overview. Aquaculture 267, 3-19.

Lan, R.X., Lee, S.I., Kim, I.H., 2016. Effects of multistrain probiotics on growth performance, nutrient digestibility, blood profiles, faecal microbial shedding, faecal score and noxious gas emission in weaning pigs. J. Anim. Physiol. Anim. Nutr. 100, 1130-1138.

Lauff, M., Hofer, R., 1984. Proteolytic enzymes in fish development and the importance of dietary enzymes. Aquaculture, 37, 335-346.

Lee, Y.J., Kim, B.K., Lee, B.H., Jo, K.I., Lee, N.K., Chung, C.H., Lee, Y.C., Lee, J.W., 2008. Purification and characterization of cellulase produced by Bacillus amyoliquefaciens DL-3 utilizing rice hull. Bioresour. Technol. 99, 378-386.

Lee, S.H., Ingale, S.L., Kim, J.S., Kim, K.H., Lokhande, A., Kim, E.K., Chae, B.J., 2014. Effects of dietary supplementation with Bacillus subtilis LS 1-2 fermentation biomass on growth performance, nutrient digestibility, cecal microbiota and intestinal morphology of weanling pig. Anim. Feed Sci. Technol. 188, 102-110.

Leenhouwers, J.I., Ortega, R.C., Verreth, J.A.J., Schrama, J.W., 2007a. Digesta characteristics in relation to nutrient digestibility and mineral absorption in Nile tilapia (Oreochromis niloticus L.) fed cereal grains of increasing viscosity. Aquaculture 273, 556-565. 
Leenhouwers, J.I., ter Veld, M., Verreth, J.A., Schrama, J.W., 2007b. Digesta characteristiscs and performance of African catfish (Clarias gariepinus) fed cereal grains that differ in viscosity. Aquaculture 264, 330-341.

Leenhouwers, J., Pellikaan, W., Huizing, H., Coolen, R., Verreth, J.A., Schrama, J.W., 2008. Fermentability of carbohydrates in an in vitro batch culture method using inocula from Nile tilapia (Oreochromis niloticus) and European sea bass (Dicentrarchus labrax). Aquac. Nutr. 14, 523-532.

Lemos, D., Tacon, A.G., 2017. Use of phytases in fish and shrimp feeds: a review. Rev. Aquac. 9, 266282.

Lenartovicz, V., Marques De Souza, C.G., Moreira, F.G., Peralta, R.M., 2002. Temperature effect in the production of multiple xylanases by Aspergillus fumigatus. J. Basic Microbiol. 42, 388-395.

$\mathrm{Li}, \mathrm{J} ., \mathrm{Li}, \mathrm{J} ., \mathrm{Wu}, \mathrm{T} ., 2009$. Effects of non-starch polysaccharides enzyme, phytase and citric acid on activities of endogenous digestive enzymes of tilapia (Oreochromis niloticus $\times$ Oreochromis aureus). Aquac. Nutr. 15, 415-420.

Li, Y., Bruni, L., Jaramillo-Torres, A., Gajardo, K., Kortner, T.M., Krogdahl, Å., 2020. Differential Response of Digesta- and Mucosa-Associated Intestinal Microbiota to Dietary Black Soldier Fly (Hermetia illucens) Larvae Meal in Seawater Phase Atlantic Salmon (Salmo salar). bioRxiv 2020.

Liebert, F., Portz, L., 2007. Different sources of microbial phytase in plant based low phosphorus diets for Nile tilapia Oreochromis niloticus may provide different effects on phytate degradation. Aquaculture 267, 292-299.

Liebert, F., Portz, L., 2005. Nutrient utilization of Nile tilapia Oreochromis niloticus fed plant based low phosphorus diets supplemented with graded levels of different sources of microbial phytase. Aquaculture 248, 111-119.

Lin, S., Mai, K., Tan, B., 2007. Effects of exogenous enzyme supplementation in diets on growth and feed utilization in tilapia, Oreochromis niloticus x O. aureus. Aquac. Res. 38, 1645-1653.

Lim, C., Lückstädt, C., Webster, C.D., Kesius, P., 2015. Organic acids and their salts. Dietary nutrients, additives, and fish health. Willey-Blackwell, Hoboken, NJ, USA, 305-320.

Liu, S.Q., 2003. Practical implications of lactate and pyruvate metabolism by lactic acid bacteria in food and beverage fermentations. Int. J. Food Microbiol. 83, 115-131.

Liu, H., Wang, S., Cai, Y., Guo, X., Cao, Z., Zhang, Y., Liu, S., Yuan, W., Zhu, W., Zheng, Y., 2017. Dietary administration of Bacillus subtilis HAINUP40 enhances growth, digestive enzyme activities, innate immune responses and disease resistance of tilapia, Oreochromis niloticus. Fish Shellfish Immunol. 60, 326-333.

Lu, W., Li, D., Wu, Y., 2003. Influence of water activity and temperature on xylanase biosynthesis in pilotscale solid-state fermentation by Aspergillus sulphureus. Enzyme Microb. Technol. 32, 305-311.

Lupatsch, I., Kissil, G.W., Sklan, D., 2003. Comparison of energy and protein efficiency among three fish species gilthead sea bream (Sparus aurata), European sea bass (Dicentrarchus labrax) and white grouper (Epinephelus aeneus): energy expenditure for protein and lipid deposition. Aquaculture. 225, 175-189.

Lupatsch, I., Deshev, R., Magen, I., 2010. Energy and protein demands for optimal egg production including maintenance requirements of female tilapia Oreochromis niloticus. Aquac. Res. 41, 763769.

\section{M}

Ma, T., Suzuki, Y., 2018. Dissect the mode of action of probiotics in affecting host-microbial interactions and immunity in food producing animals. Vet. Immunol. Immunopathol. 205, 35-48.

Maas, R.M., Verdegem, M.C.J, Dersjant-Li, Y., Schrama, J.W., 2018. The effect of phytase, xylanase and their combination on growth performance and nutrient utilization in Nile tilapia. Aquaculture 487, 7-14.

Maas, R.M., Verdegem, M.C.J, Schrama, J.W., 2019. Effect of non-starch polysaccharide composition and enzyme supplementation on growth performance and nutrient digestibility in Nile tilapia (Oreochromis niloticus). Aquac. Nutr. 25, 622-632

Maas, R.M., Verdegem, M.C.J, Wiegertjes, G.F., Schrama, J.W., 2020a. Carbohydrate utilisation by tilapia: a meta-analytical approach. Rev. Aquacult. 12, 1851-1866.

Maas, R.M., Verdegem, M.C.J., Stevens, T.L., Schrama, J.W., 2020b. Effect of exogenous enzymes (phytase and xylanase) supplementation on nutrient digestibility and growth performance of Nile tilapia (Oreochromis niloticus) fed different quality diets. Aquaculture 529.

Macfarlane, S., Macfarlane, G.T., 2003. Regulation of short-chain fatty acid production. Proc. Nutr. Soc. 62, 67-72.

Mainstone, C.P., Parr, W., 2002. Phosphorus in rivers-ecology and management. Sci. Total Environ. 282, 25-47.

Makled, S.O., Hamdan, A.M., El-Sayed, A.F.M., 2019. Growth Promotion and Immune Stimulation in Nile Tilapia, Oreochromis niloticus, Fingerlings Following Dietary Administration of a Novel Marine Probiotic, Psychrobacter maritimus S. Probiotics Antimicrob. Proteins 12, 365-374.

Martins, C.I.M., Eding, E.H., Verdegem, M.C.J., Heinsbroek, L.T., Schneider, O., Blancheton, J. P., Verreth, J.A.J., 2010. New developments in recirculating aquaculture systems in Europe: A perspective on environmental sustainability. Aquac. Eng. 43, 83-93. 
Mazurkiewicz, J., 2009. Utilization of domestic plant components in diets for common carp Cyprinus carpio L. Archives of Polish Fisheries 17, 5-39.

Merrifield, D.L., Dimitroglou, A., Foey, A., Davies, S.J., Baker, R.T., Bøgwald, J., Ringø, E., 2010. The current status and future focus of probiotic and prebiotic applications for salmonids. Aquaculture 302, 1-18.

Metzler-Zebeli, B.U., Hooda, S., Pieper, R., Zijlstra, R.T., van Kessel, A.G., Mosenthin, R., Gänzle, M.G., 2010. Nonstarch polysaccharides modulate bacterial microbiota, pathways for butyrate production, and abundance of pathogenic Escherichia coli in the pig gastrointestinal tract. Appl. Environ. Microbiol. 76, 3692-3701.

Montagne, L., Pluske, J., Hampson, D., 2003. A review of interactions between dietary fibre and the intestinal mucosa, and their consequences on digestive health in young non-ruminant animals. Anim. Feed Sci. Technol. 108, 95-117.

Moraïs, S., Mizrahi, I., 2019. Islands in the stream: from individual to communal fiber degradation in the rumen ecosystem. FEMS Microbiol. Rev. 43, 362-379.

Mountfort, D.O., Campbell, J., Clements, K.D., 2002. Hindgut fermentation in three species of marine herbivorous fish. Appl. Environ. Microbiol. 68, 1374-1380.

Mroz, Z., Moeser, A., Vreman, K., Van Diepen, J., Van Kempen, T., Canh, T., Jongbloed, A., 2000. Effects of dietary carbohydrates and buffering capacity on nutrient digestibility and manure characteristics in finishing pigs. J. Anim. Sci. 78, 3096-3106.

Mountzouris, K.C., Tsitrsikos, P., Palamidi, I., Arvaniti, A., Mohnl, M., Schatzmayr, G., Fegeros, K., 2010. Effects of probiotic inclusion levels in broiler nutrition on growth performance, nutrient digestibility, plasma immunoglobulins, and cecal microflora composition. Poult. Sci. 89, 58-67.

\section{$\mathbf{N}$}

National Research Council (NRC), 2011. Nutrient Requirements of Fish and Shrimp. The National Academies Press, Washington DC.

Nawaz, A., Irshad, S., Hoseinifar, S.H., Xiong, H., 2018. The functionality of prebiotics as immunostimulant: Evidences from trials on terrestrial and aquatic animals. Fish Shellfish Immunol. 76, 272-278.

Nayak, S.K., 2010. Probiotics and immunity: a fish perspective. Fish Shellfish Immun. 29, 2-14.

Naylor, R.L., Hardy, R.W., Bureau, D.P., Chiu, A., Elliott, M., Farrell, A.P., Hua, K., 2009. Feeding aquaculture in an era of finite resources. Proc. Natl. Acad. Sci. 106, 15103-15110.

Newaj-Fyzul, A., Al-Harbi, A., Austin, B., 2014. Developments in the use of probiotics for disease control in aquaculture. Aquaculture 431, 1-11.

Nishida, A.H., Ochman, H., 2018. Rates of gut microbiome divergence in mammals. Mol. Ecol. 27, 18841897.

Nolan, K.B., Duffin, P.A., McWeeny, D.J., 1987. Effects of phytate on mineral bioavailability. In vitro studies on $\mathrm{Mg}^{2+}, \mathrm{Ca}^{2+}, \mathrm{Fe}^{3+}, \mathrm{Cu}^{2+}$ and $\mathrm{Zn}^{2+}\left(\right.$ also $\left.\mathrm{Cd}^{2+}\right)$ solubilities in the presence of phytate. J. Sci. Food Agric. 40, 79-85.

\section{$\mathbf{O}$}

Obirikorang, K.A., Amisah, S., Fialor, S.C., Skov, P.V., 2015. Digestibility and postprandial ammonia excretion in Nile tilapia (Oreochromis niloticus) fed diets containing different oilseed by-products. Aquac. Int. 23, 1249-1260.

Obersteiner, M., Peñuelas, J., Ciais, P., Van Der Velde, M., Janssens, I.A., 2013. The phosphorus trilemma. Nat. Geosci. 6, 897.

Officer, D., 2000. Feed enzymes. Farm Animal Metabolism and Nutrition, 405-426.

Ogunkoya, A.E., Page, G.I., Adewolu, M.A., Bureau, D.P., 2006. Dietary incorporation of soybean meal and exogenous enzyme cocktail can affect physical characteristics of faecal material egested by rainbow trout (Oncorhynchus mykiss). Aquaculture 254, 466-475.

Oliva-Teles, A., Pereira, J.P., Gouveia, A., Gomes, E., 1998. Utilisation of diets supplemented with microbial phytase by seabass (Dicentrarchus labrax) juveniles. Aquat. Living Resour. 11, 255-259.

Oliva-Teles, A., Enes, P., Peres, H., 2015. Replacing fishmeal and fish oil in industrial aquafeeds for carnivorous fish, Feed and feeding practices in aquaculture. Woodhead Publishing, 203-233.

Opstvedt, J., Aksnes, A., Hope, B., Pike, I. H., 2003. Efficiency of feed utilization in Atlantic salmon (Salmo salar L.) fed diets with increasing substitution of fish meal with vegetable proteins. Aquaculture $221,365-379$.

Oliveira Coutinho, de, J.J., Neira, L.M., de Sandre, L.C.G., da Costa, J.I., Martins, M.I.E.G., Portella, M.C., Carneiro, D.J., 2018. Carbohydrate-to-lipid ratio in extruded diets for Nile tilapia farmed in net cages. Aquaculture 497, 520-525.

Oliveira, M., Machado, A.V., 2013. The role of phosphorus on eutrophication: a historical review and future perspectives. Environ. Technol. Rev. 2, 117-127.

Øverland, M., Sørensen, M., Storebakken, T., Penn, M., Krogdahl, A.,, Skrede, A., 2009. Pea protein concentrate substituting fish meal or soybean meal in diets for Atlantic salmon (Salmo salar)effect on growth performance, nutrient digestibility, carcass composition, gut health, and physical feed quality. Aquaculture 288, 305-311. 


\section{P}

Pahlow, M., van Oel, P.R., Mekonnen, M.M., Hoekstra, A.Y., 2015. Increasing pressure on freshwater resources due to terrestrial feed ingredients for aquaculture production. Sci. Total Environ. 536, 847-857.

Papatryphon, E., Howell, R.A., Soares Jr, J.H., 1999. Growth and mineral absorption by striped bass Morone saxatilis fed a plant feedstuff based diet supplemented with phytase. J. World Aquacult. Soc. 30, 161-173.

Pascoal, L.A.F., Thomaz, M.C., Watanabe, P.H., Ruiz, U.D.S., Ezequiel, J.M.B., Amorim, A.B., Daniel, E., Masson, G.C.I., 2012. Fiber sources in diets for newly weaned piglets. Rev. Bras. de Zootec. 41, 636-642.

Pen-Hsing, T., Shi-Yen S., 1993. Carbohydrate utilization versus body size in tilapia Oreochromis niloticus $\times$ O. aureus. Comp. Biochem. Physiol. Part A Physiol. 104, 585-588.

Pérez, T., Balcázar, J.L., Ruiz-Zarzuela, I., Halaihel, N., Vendrell, D., De Blas, I., Múzquiz, J.L., 2010. Hostmicrobiota interactions within the fish intestinal ecosystem. Mucosal immunol. 3, 355-360.

Petit, J., Bailey, E.C., Wheeler, R.T., de Oliveira, C.A., Forlenza, M., Wiegertjes, G.F., 2019a. Studies Into $\beta$-Glucan Recognition in Fish Suggests a Key Role for the C-Type Lectin Pathway. Front. Immunol. 10, 280.

Petit, J., Embregts, C.W., Forlenza, M., Wiegertjes, G.F., 2019b. Evidence of trained immunity in a fish: conserved features in carp macrophages. J. Immunol. 203, 216-224.

Petit, J., Wiegertjes, G.F., 2016. Long-lived effects of administering $\beta$-glucans: indications for trained immunity in fish. Dev. Comp. Immunol. 64, 93-102.

Peuhkuri, T., 2002. Knowledge and interpretation in environmental conflict: Fish farming and eutrophication in the Archipelago Sea, SW Finland. Landsc. Urban Plan. 61, 157-168.

Phillips Jr, A.M, Brockway, D.R., 1956. The nutrition of trout: II. Protein and carbohydrate. Progress. Fish Cult. 18, 159-164.

Phan, L., Groot, R., Konnert, G.D.P., Masagounder K., Figueiredo-Silva, A.C., Glencross, B.D., Schrama, J.W., 2019. Differences in energy utilisation efficiencies of digestible macronutrients in common carp (Cyprinus carpio) and barramundi (Lates calcarifer). Aquaculture 511, 734238.

Piazzon, M.C., Calduch-Giner, J.A., Fouz, B., Estensoro, I., Simo-Mirabet, P., Puyalto, M., Karalazos, V., Palenzuela, O., Sitja-Bobadilla, A., Perez-Sanchez, J., 2017. Under control: how a dietary additive can restore the gut microbiome and proteomic profile, and improve disease resilience in a marine teleostean fish fed vegetable diets. Microbiome 5, 164.

Pimentel-Rodrigues, A., Oliva-Teles, A., 2007. Phosphorus availability of inorganic phosphates and fish meals in European sea bass (Dicentrarchus labrax L.) juveniles. Aquaculture 267, 300-307.

Polizeli, M.L.T.M., Rizzatti, A.C.S., Monti, R., Terenzi, H.F., Jorge, J.A., Amorim, D.S., 2005. Xylanases from fungi: properties and industrial applications. Appl. Microbiol. Biotechnol. 67, 577-591.

Portz, L., Liebert, F., 2004. Growth, nutrient utilization and parameters of mineral metabolism in Nile tilapia Oreochromis niloticus (Linnaeus, 1758) fed plant-based diets with graded levels of microbial phytase. J. Anim. Physiol. Anim. Nutr. 88, 311-320.

Prabhu, P.A.J., Schrama, J.W., Kaushik, S.J., 2013. Quantifying dietary phosphorus requirement of fish-a meta-analytic approach. Aquac. Nutr. 19, 233-249.

Prabhu, P.A.J., Kaushik, S.J., Geurden, I., Stouten, T., Fontagne-Dicharry, S., Veron, V., Schrama, J.W., 2017. Water exchange rate in RAS and dietary inclusion of micro-minerals influence growth, body composition and mineral metabolism in common carp. Aquaculture 471, 8-18.

Prabu, E., Rajagopalsamy, C.B.T., Ahilan, B., Jeevagan, I.J.M.A., Renuhadevi, M., 2019. Tilapia-an excellent candidate species for world aquaculture: a review. Annu. Res. Rev. Biol. 1-14.

Q

Qaisrani, S., Moquet, P., Van Krimpen, M., Kwakkel, R., Verstegen, M., Hendriks, W., 2014. Protein source and dietary structure influence growth performance, gut morphology, and hindgut fermentation characteristics in broilers. Poult. Sci. 93, 3053-3064.

\section{$\mathbf{R}$}

Rajkumar, M., Pandey, P.K., Aravind, R., Vennila, A., Bharti, V., Purushothaman, C.S., 2016. Effect of different biofloc system on water quality, biofloc composition and growth performance in Litopenaeus vannamei (Boone, 1931). Aquac. Res. 47, 3432-3444.

Ravindran, V.B.W.K.E., Bryden, W.L., Kornegay, E.T., 1995. Phytates: occurrence, bioavailability and implications in poultry nutrition. Avian Poult. Biol. Rev. 6, 125-143.

Reda, R.M., Mahmoud, R., Selim, K.M., El-Araby, I.E., 2016. Effects of dietary acidifiers on growth, hematology, immune response and disease resistance of Nile tilapia, Oreochromis niloticus. Fish Shellfish Immunol. 50, 255-262.

Reda, R.M., Selim, K.M., 2015. Evaluation of Bacillusamyloliquefaciens on the growth performance, intestinal morphology, hematology and body composition of Nile tilapia, Oreochromis niloticus. Aquac. Int. 23, 203-217. 
Richardson, N.L., Higgs, D.A., Beames, R.M., Mcbride, J.R., 1985. Influence of dietary calcium, phosphorus, zinc and sodium phytate level on cataract incidence, growth and histopathology in juvenile chinook salmon (Oncorhynchus tshawytscha). J. Nutr. 115, 553-567.

Riche, M. Brown, P.B., 1996. Availability of phosphorus from feedstuffs fed to rainbow trout, Oncorhynchus mykiss. Aquaculture 142, 269-282.

Riche, M., Garling Jr, D., 2004. Effect of phytic acid on growth and nitrogen retention in tilapia Oreochromis niloticus L. Aquac. Nutr. 10, 389-400.

Ridha, M.T., Azad, I.S., 2012. Preliminary evaluation of growth performance and immune response of Nile tilapia Oreochromis niloticus supplemented with two putative probiotic bacteria. Aquac. Res. 43, 843-852.

Rodrigues, A.P.O., Gominho-Rosa, M., Cargnin-Ferreira, E., de Francisco, A., Fracalossi, D.M., 2012. Different utilization of plant sources by the omnivores jundiá catfish (Rhamdia quelen) and Nile tilapia (Oreochromis niloticus). Aquac. Nutr. 18, 65-72.

Romero, L.F., Parsons, C.M., Utterback, P.L, Plumstead, P.W, Ravindran, V., 2013. Comparative effects of dietary carbohydrases without or with protease on the ileal digestibility of energy and amino acids and AMEn in young broilers. Anim. Feed Sci. Technol. 181, 35-44.

Russell, J., 1998. The importance of $\mathrm{pH}$ in the regulation of ruminal acetate to propionate ratio and methane production in vitro. J. Dairy Sci. 81, 3222-3230.

S

Saputra, F., Shiu, Y.L., Chen, Y.C., Puspitasari, A.W., Danata, R.H., Liu, C.H., Hu, S.Y., 2016. Dietary supplementation with xylanase-expressing $B$. amyloliquefaciens R8 improves growth performance and enhances immunity against Aeromonas hydrophila in Nile tilapia (Oreochromis niloticus). Fish Shellfish Immun. 58, 397-405.

Saravanan, S., Geurden, I., Figueiredo-Silva, A., Kaushik, S.J., Haidar, M., Verreth, J.A.J., Schrama, J.W, 2012. Control of voluntary feed intake in fish: a role for dietary oxygen demand in Nile tilapia (Oreochromis niloticus) fed diets with different macronutrient profiles. Br. J. Nutr. 108, 15191529.

Saravanan, S., Geurden, I., Orozco, Z.G.A., Kaushik, S.J., Verreth, J.A.J., Schrama, J.W., 2013. Dietary electrolyte balance affects the nutrient digestibility and maintenance energy expenditure of Nile tilapia. Br. J. Nutr. 110, 1948-1957.

Saravanan, S., Schrama, J.W., Figueiredo-Silva, A.C., Kaushik, S.J., Verreth, J.A.J., Geurden, I., 2012. Constraints on energy intake in fish: the link between diet composition, energy metabolism, and energy intake in rainbow trout. PLoS One 7, e34743.

Satoh, S., Takanezawa, M., Akimoto, A., Kiron, V., Watanabe, T., 2002. Changes of phosphorus absorption from several feed ingredients in rainbow trout during growing stages and effect of extrusion of soybean meal. Fish. Sci. 68, 325-331.

Schäfer, A., Koppe, W.M., Meyer-Burgdorff, K.H., Günther, K.D., 1995. Effects of a microbial phytase on the utilization of native phosphorus by carp in a diet based on soybean meal. Water Sci. Technol. $31,149-155$.

Scheppach, W., 1994. Effects of short chain fatty acids on gut morphology and function. Gut, 35(1 Suppl), S35-S38.

Schneider, O., Amirkolaie, A.K., Vera-Cartas, J., Eding, E.H., Schrama, J.W., Verreth, J.A.J., 2004. Digestibility, faeces recovery, and related carbon, nitrogen and phosphorus balances of five feed ingredients evaluated as fishmeal alternatives in Nile tilapia, Oreochromis niloticus L. Aquac. Res. $35,1370-1379$.

Schneider, O., Amirkolaie, A.K., Vera-Cartas, J., Eding, E.H., Schrama, J.W., Verreth, J.A.J., 2004. Digestibility, faeces recovery, and related carbon, nitrogen and phosphorus balances of five feed ingredients evaluated as fishmeal alternatives in Nile tilapia, Oreochromis niloticus L. Aquac. Res. $35,1370-1379$.

Schrama, J.W., Saravanan, S., Geurden, I., Heinsbroek, L., Kaushik, S.J., Verreth, J.A.J., 2012. Dietary nutrient composition affects digestible energy utilisation for growth: a study on Nile tilapia (Oreochromis niloticus) and a literature comparison across fish species. Br. J. Nutr. 108, 277-289.

Schryver, De, P., Crab, R., Defoirdt, T., Boon, N., Verstraete, W., 2008. The basics of bio-flocs technology: the added value for aquaculture. Aquaculture 277, 125-137.

Seidavi, A., Dadashbeiki, M., Alimohammadi-Saraei, M.H., van den Hoven, R., Payan-Carreira, R., Laudadio, V., Tufarelli, V., 2017. Effects of dietary inclusion level of a mixture of probiotic cultures and enzymes on broiler chickens immunity response. Environ. Sci. Pollut. Res. 24, 4637-4644.

Selim, K.M., Reda, R.M., 2015. Improvement of immunity and disease resistance in the Nile tilapia, Oreochromis niloticus, by dietary supplementation with Bacillus amyloliquefaciens. Fish Shellfish Immunol. 44, 496-503.

Shepherd, C., Jackson, A., 2013. Global fishmeal and fish-oil supply: inputs, outputs and marketsa. J. Fish Biol. 83, 1046-1066.

Silva, T.F.A., Petrillo, T.R., Yunis-Aguinaga, J., Marcusso, P.F., da Silva Claudiano, G., de Moraes, F.R., de Moraes, J.R.E., 2015. Effects of the probiotic Bacillus amyloliquefaciens on growth performance, hematology and intestinal morphometry in cage-reared Nile tilapia. Lat. Am. J. Aquat. Res. 43, 963-971. 
Sinha, A.K., Kumar, V., Makkar, H.P., De Boeck, G., Becker, K., 2011. Non-starch polysaccharides and their role in fish nutrition-A review. Food Chem. 127, 1409-1426.

Sintayehu, A., Mathies, E., Meyer-Burgdorff, K.H., Rosenow, H., Günther, K.D., 1996. Apparent digestibilities and growth experiments with tilapia (Oreochromis niloticus) fed soybean meal, cottonseed meal and sunflower seed meal. J. Appl. Ichthyol. 12, 125-130.

Skoglund, E., Carlsson, N.G., Sandberg, A.S., 2009. Phytate. HEALTHGRAIN methods: analysis of bioactive components in small grain cereals, 129-139.

Smits, C.H., Annison, G., 1996. Non-starch plant polysaccharides in broiler nutrition-towards a physiologically valid approach to their determination. Worlds Poult. Sci. J. 52, 203-221.

Spinelli, J., Houle, C.R., Wekell, J.C., 1983. The effect of phytates on the growth of rainbow trout (Salmo gairdneri) fed purified diets containing varying quantities of calcium and magnesium. Aquaculture 30, 71-83.

Staessen, T.W.O., Verdegem, M. C. J., Weththasinghe, P., Schrama, J. W., 2020. The effect of dietary nonstarch polysaccharide level and bile acid supplementation on fat digestibility and the bile acid balance in rainbow trout (Oncorhynchus mykiss). Aquaculture 735174.

Stone, D., Allan, G., Anderson, A., 2003. Carbohydrate utilization by juvenile silver perch, Bidyanus bidyanus (Mitchell). IV. Can dietary enzymes increase digestible energy from wheat starch, wheat and dehulled lupin?. Aquac. Res. 34, 135-147.

Stone, D.A.J., 2003. Dietary Carbohydrate Utilization by Fish. Rev. Fish. Sci. 11, 337-369.

Storebakken, T., Austreng, E., 1987. Ration level for salmonids: I. Growth, survival, body composition, and feed conversion in Atlantic salmon fry and fingerlings. Aquaculture 60, 189-206.

Storebakken, T., Shearer, K.D., Roem, A.J., 1998. Availability of protein, phosphorus and other elements in fish meal, soy-protein concentrate and phytase-treated soy-protein-concentrate-based diets to Atlantic salmon, Salmo salar. Aquaculture 161, 365-379.

Sugita, H., Miyajima, C., Deguchi, Y., 1991. The vitamin B12-producing ability of the intestinal microflora of freshwater fish. Aquaculture 92, 267-276.

Sugita, H., Shibuya, K., Shimooka, H., Deguchi, Y., 1996. Antibacterial abilities of intestinal bacteria in freshwater cultured fish. Aquaculture 145, 195-203.

Sugiura, S., Gabaudan, J., Dong, F., Hardy, R., 2001. Dietary microbial phytase supplementation and the utilization of phosphorus, trace minerals and protein by rainbow trout [(Oncorhynchus mykiss (Walbaum)] fed soybean meal-based diets. Aquac. Res. 32, 583-592.

Sugiura, S.H., Ferraris, R.P., 2004. Dietary phosphorus-responsive genes in the intestine, pyloric ceca, and kidney of rainbow trout. Am. J. Physiol. Regul. Integr. Comp. Physiol. 287, 541-550.

Svihus, B., 2010. Effect of digestive tract conditions, feed processing and ingredients on response to NSP enzymes. Enzymes in farm animal nutrition 129.

\section{T}

Tacon, A.G., Hasan, M.R., Metian, M., 2011. Demand and supply of feed ingredients for farmed fish and crustaceans: trends and prospects. FAO Fisheries and Aquaculture technical paper, (564), I.

Tacon, A.G.J., Metian, M., 2015. Feed Matters: Satisfying the Feed Demand of Aquaculture. Rev. Fish. Sci. Aquac. 23, 1-10.

Tahir, M., Saleh, F., Ohtsuka, A., Hayashi, K., 2005. Synergistic effect of cellulase and hemicellulase on nutrient utilization and performance in broilers fed a corn-soybean meal diet. Anim. Sci. J. 76, 559-565.

Tahoun, A., Hammouda, Y., 2009. Effect of adding commercial phytase to DDGS based diets on the performance and feed utilization of nile tilapia (Oreochromis niloticus) fingerlings. Am.-Eurasian $\mathrm{J}$. Agric. Environ. Sci. 5, 550-555.

Tangendjaja, B., 2015. 6 - Quality control of feed ingredients for aquaculture A2 - Davis, D. Allen Feed and Feeding Practices in Aquaculture (141-169). Oxford: Woodhead Publishing.

Teuling, E., Wierenga, P.A., Agboola, J.O., Gruppen, H., Schrama, J.W., 2019. Cell wall disruption increases bioavailability of Nannochloropsis gaditana nutrients for juvenile Nile tilapia (Oreochromis niloticus). Aquaculture 499, 269-282.

Tran-Ngoc, K.T., Dinh, N.T., Nguyen, T.H., Roem, A.J., Schrama, J.W., Verreth, J.A.J., 2016. Interaction between dissolved oxygen concentration and diet composition on growth, digestibility and intestinal health of Nile tilapia (Oreochromis niloticus). Aquaculture 462, 101-108.

Troell, M., Naylor, R.L., Metian, M., Beveridge, M., Tyedmers, P.H., Folke, C., Ehrlich, P.R., 2014. Does aquaculture add resilience to the global food system? Proc. Natl. Acad. Sci. 111, 13257-13263.

Tyrrell, T., 1999. The relative influences of nitrogen and phosphorus on oceanic primary production. Nature $400,525-531$.

U

Ueki, A., Akasaka, H., Suzuki, D., Ueki, K., 2006. Paludibacter propionicigenes gen. nov., sp. nov., a novel strictly anaerobic, Gram-negative, propionate-producing bacterium isolated from plant residue in irrigated rice-field soil in Japan. Int. J. Syst. Evol. Microbiol. 56, 39-44.

United Nations, 2015. World population prospects: The 2015 revision. United Nations Econ. Soc. Aff. 33, $1-66$. 
Urán, P.A., Schrama, J.W., Rombout, J.H.W.M., Obach, A., Jensen, L., Koppe, W., Verreth, J.A.J., 2008. Soybean meal-induced enteritis in Atlantic salmon (Salmo salar L.) at different temperatures. Aquacult. Nutr. 14, 324-330.

\section{$\mathbf{W}$}

Wallace, J.L., Murray, F.J., Little, D.C., 2016. Effects of $\beta$-xylanase and 6-phytase on digestibility, trace mineral utilisation and growth in juvenile red tilapia, Oreochromis niloticus (Linnaeus, 1758) $\times 0$. mossambicus (Peters, 1852), fed declining fishmeal diets. J. Appl. Ichthyol. 32, 471-479.

Wang, Y., Liu, Y.J., Tian, L.X., Du, Z.Y., Wang, J.T., Wang, S., Xiao, W.P, 2005. Effects of dietary carbohydrate level on growth and body composition of juvenile tilapia, Oreochromis niloticus $\times 0$. aureus. Aquac. Res. 36, 1408-1413.

Wang, Y.B., Tian, Z.Q., Yao, Li, W.F., 2008. Effect of probiotics, Enteroccus faecium, on tilapia (Oreochromis niloticus) growth performance and immune response. Aquaculture 277, 203-207.

Wang, Y., Song, J., Zhai, Y., Zhang, C., Gerritsen, J., Wang, H., Chen, X., Li, Y., Zhao, B., Zhao, B., Ruan, Z., 2015. Romboutsia sedimentorum sp. nov., isolated from an alkaline-saline lake sediment and emended description of the genus Romboutsia. Int. J. Syst. Evol. Microbiol. 65, 1193-1198.

Wang, M., Lu, M., 2016. Tilapia polyculture: a global review. Aquac. Res. 47, 2363-2374.

Wiese, M., 2019. The potential of pectin to impact pig nutrition and health: feeding the animal and its microbiome. FEMS Microbiol. Lett. 366, fnz029.

Wealleans, A., Walsh, M., Romero, L., Ravindran, V., 2017. Comparative effects of two multi-enzyme combinations and a Bacillus probiotic on growth performance, digestibility of energy and nutrients, disappearance of non-starch polysaccharides, and gut microflora in broiler chickens. Poult. Sci. $96,4287-4297$.

Wentzel, A.S., Janssen, J.J., De Boer, V.C., Van Veen, W.G., Forlenza, M., Wiegertjes, G.F., 2020. Fish Macrophages Show Distinct Metabolic Signatures Upon Polarization. Front. Immunol. 11.

Wilson, R., 1994. Utilization of dietary carbohydrate by fish. Aquaculture 124, 67-80.

Williams, B.A., Verstegen, M.W., Tamminga, S., 2001. Fermentation in the large intestine of singlestomached animals and its relationship to animal health. Nutr. Res. Rev. 14, 207-228.

Williams, B.A., Grant, L.J., Gidley, M.J., 2017. Gut fermentation of dietary fibres: physico-chemistry of plant cell walls and implications for health. Int. J. Mol. Sci. 18, 2203.

Windell, J. T., Foltz, J. W., Sarokon, J. A., 1978. Effect of fish size, temperature, and amount fed on nutrient digestibility of a pelleted diet by rainbow trout, Salmo gairdneri. Trans. Am. Fish. Soc. 613-616.

Woyengo, T., Nyachoti, C., 2011. Supplementation of phytase and carbohydrases to diets for poultry. Can. J. Anim. Sci. 91, 177-192.

\section{$\mathbf{X}$}

Xu, R., Li, M., Wang, T., Zhao, Y.W., Shan, C.J., Qiao, F., Chen, L.Q., Du, Z.Y., Zhang, M.L., 2020. A strain affiliated to Bacillus amyloliquefaciens alleviates high-carbohydrate diet- induced metabolic syndrome by restoration of acetate-producing bacteria in fish intestines, Research Square.

\section{$\mathbf{Y}$}

Yang, G., Peng, M., Tian, X., Dong, S., 2017. Molecular ecological network analysis reveals the effects of probiotics and florfenicol on intestinal microbiota homeostasis: An example of sea cucumber. Sci Rep. 7, 4778.

Yao, Y.F., Jiang, M., Wen, H., Wu, F., Liu, W., Tian, J., Yang, C.G., 2014. Dietary phosphorus requirement of GIFT strain of Nile tilapia Oreochromis niloticus reared in freshwater. Aquac. Nutr. 20, 273-280.

Yigit, N., Olmez, M., 2011. Effects of cellulase addition to canola meal in tilapia (Oreochromis niloticus L.) diets. Aquac. Nutr. 17, 494-500.

Yildirim, Y.B., Turan, F., 2010. Effects of exogenous enzyme supplementation in diets on growth and feed utilization in African catfish, Clarias gariepinus. J. Anim. Vet. Adv. 9, 327-331.

Yu, S., Cowieson, A., Gilbert, C., Plumstead, P., Dalsgaard, S., 2012. Interactions of phytate and myoinositol phosphate esters (IP) including IP isomers with dietary protein and iron and inhibition of pepsin. J. Anim. Sci. 90, 1824-1832.

\section{$\mathbf{Z}$}

Zanten, van, H.H., Herrero, M., Van Hal, O., Röös, E., Muller, A., Garnett, T., De Boer, I.J., 2018. Defining a land boundary for sustainable livestock consumption. Glob. Chang. Biol. 24, 4185-4194.

Zhang, L., Zhang, L., Zeng, X., Zhou, L., Cao, G.,Yang, C., 2016. Effects of dietary supplementation of probiotic, Clostridium butyricum, on growth performance, immune response, intestinal barrier function, and digestive enzyme activity in broiler chickens challenged with Escherichia coli K88. J. Anim. Sci. Biotechnol. 7.1, 3.

Zhang, C., Rahimnejad, S., Wang, Y.R., Lu, K., Song, K., Wang, L., Mai, K., 2018. Substituting fish meal with soybean meal in diets for Japanese seabass (Lateolabrax japonicus): Effects on growth, digestive enzymes activity, gut histology, and expression of gut inflammatory and transporter genes. Aquaculture 483, 173-182. 
Zheng, C.C., Wu, J.W., Jin, Z.H., Ye, Z.F., Yang, S., Sun, Y.Q., Fei, H., 2020. Exogenous enzymes as functional additives in finfish aquaculture. Aquacult. Nutr. 26, 213-224.

Zhou, Y., Yuan, X., Liang, X.F., Fang, L., Li, J., Guo, X., Bai, X., He, S., 2013. Enhancement of growth and intestinal flora in grass carp: the effect of exogenous cellulase. Aquaculture 416, 1-7. 



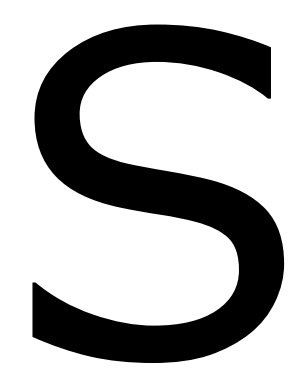

Summary (English)

Samenvatting (Nederlands) 


\section{Summary}

With the expected further growth of aquaculture and the increasing demand for aquafeeds, the trend for reducing levels of fishmeal and fish-oil in these feeds will continue. The use of lower quality ingredients for many fish species, including tilapia, will increase in the future, leading to increased dietary levels of antinutritional factors, including non-starch polysaccharides (NSP) and phytate. Both NSP and phytate are considered indigestible, as the enzymes needed to breakdown their bonds are scarce or non-existent in fish. Supplementing feeds with dietary enzymes and probiotics can reduce the antinutritional effects of phytate and NSP. However, compared to other monogastric animals, studies on fish using phytase and NSP-degrading enzymes are scarce. Likewise, multiple studies have been performed, both on broilers and pigs on the improvement of nutrient bioavailability with the use of probiotics. However, for fish similar studies are few in number; little is currently known as to how probiotics can improve nutrient bioavailability of plant-based aquafeeds. In addition, it is expected that in fish there may be synergy between different enzymes, and between enzymes and probiotics, but this has not yet been assessed. Therefore the aim of this thesis is to assess the potential of dietary enzyme and probiotic supplementation in improving the nutritional value of low quality feed ingredients, using Nile tilapia as a model species.

The first objective was to investigate, from the literature, what is known about dietary carbohydrates, in particular NSP, for Nile tilapia (Chapter 2). Besides a qualitative approach, meta-analysis was performed on available data from tilapia digestibility studies, allowing for the quantification and estimation of carbohydrate and NSP digestibility. The meta-analyses showed that across cases, an average NSP digestibility of $24.3 \%$ was calculated. Out of all cases, $88 \%$ of them showed positive NSP digestibility, which is linked to fermentation in the gut. The metaanalyses confirm the negative effect NSP has on tilapia performance and nutrient digestibility (crude protein, fat and energy). NSP digestibility was shown to contribute to energy digestibility in tilapia.

The next two chapters (Chapter 3 and $\mathbf{4}$ ) explored whether exogenous enzymes (phytase and xylanase) could improve NSP and phosphorus (P) digestibility and thereby enhance growth; and whether the impact of the enzymes is dependent on diet-related factors. Chapter $\mathbf{3}$ investigated whether the effect of enzyme supplementation on performance and nutrient digestibility is dependent on the type of ingredient(s) used. This was done by incorporating wheat bran, sunflower meal and citrus pulp to a reference diet. These ingredients are known to be rich in different types of NSP. Enzymes (phytase and xylanase), when added to the diet, improved the growth of fish fed the wheat bran and sunflower meal diets, while fish fed the reference diet (low in NSP) and citrus pulp diet (rich in pectins) did not benefit. The observed interaction effect on growth, as well as energy and $P$ digestibility, indicates that the effectiveness of the enzymes was dependent on the NSP-rich ingredient used and thus the composition of the NSP fraction. Chapter 
4 examined whether the effect of phytase and xylanase supplementation on growth and nutrient digestibility was dependent on diet quality (dietary levels of NSP and phytate). The difference in quality (two diets) was created using differing levels of low quality ingredients. The effect of phytase on (improved) growth and nutrient digestibility was independent of diet quality, whereas the effect of xylanase on (improved) nutrient digestibility was dependent on diet quality.

In both chapters 3 and 4, the enzymes phytase and xylanase were effective in improving nutrient availability and growth. In broilers, synergy between enzymes has been observed, likewise synergy between different types of enzymes in fish can also be expected. Chapter 5, explored the main effects of phytase and xylanase, as well as the interaction (synergy) between these two enzymes. Growth was affected by the interaction between phytase and xylanase supplementation, showing a synergy between these enzymes. Growth with the control diet (no enzymes) and xylanase diet was similar, whereas fish fed the diet with phytase had improved growth. The effect of phytase on growth was further enhanced when xylanase was supplemented. Both xylanase and phytase were shown to enhance nutrient digestibility, but in contrast to growth, there was no significant interaction effect (synergy) on digestibility. The nitrogen balance showed that the effect of the synergy on growth was predominantly due to the significant effect of phytase and xylanase on protein retention. Chapter 6 investigated whether $\beta$-glucanase, another NSP-degrading enzyme, can effect growth and nutrient digestibility, and whether it interacts with phytase and xylanase. However, in this study there were no effects of the enzymes, xylanase and $\beta$-glucanase, nor their combinations, on growth performance. Only phytase enhanced the digestibility of $P$, calcium and the ash fraction, besides that, the enzymes did not affect digestibility. A comparison with the results of the wheat bran diet (Chapter 3 ) was made (same diet and enzyme treatments), in order to elaborate on the minor effects of enzyme supplementation in the present study. The responses of fish fed diets supplemented with phytase and xylanase were comparable between chapters. However, the control treatment without enzyme supplementation (Chapter 6) had a lower FCR, a higher dry matter and a higher NSP digestibility, compared to chapter 3. This suggests that, over time, diet-related factors affected the control diet and thus the repeatability of the study. It is likely that in the present study, and other studies that do not show improvement in growth with the use of enzymes, the control diets were not sufficiently limiting in nutrients for growth.

Probiotics are widely used to improve fish health and disease resistance. In addition, probiotics have been shown to improve nutrient utilisation and feed efficiency, resulting in improved growth. However, studies combining both probiotics and enzymes are scarce in fish. Chapter $\mathbf{7}$ assessed the impact of enzymes and probiotics, as well as their synergistic effect. Both enzymes and probiotics improved growth when applied individually. The combination of enzymes and probiotics showed an interaction effect on growth. Enzymes improved growth to a greater extent than probiotics, whereas the combination of enzymes and probiotics did not further enhance growth. Enzymes improved the digestibility of 
all nutrients, whereas probiotics enhanced mainly fat digestibility. Additionally, probiotic supplementation reduced the energy requirements for maintenance. Individually, both enzymes and probiotics had positive effects on the measured parameters, however, a synergy and also complementary mode of action between enzymes and probiotics was mostly absent. From the same study, samples of the gut and the digesta along the gut were collected. These were used to study the effect of enzymes and probiotics on the digestion kinetics along the gastrointestinal tract (GI-tract), as well as their impact on microbiota in the distal gut (Chapter 8). Differences in $P$ availability with enzyme supplementation were present in the proximal part of the GI-tract. This indicates that the enzymes were active (breaking down phytate) in the stomach and potentially in the GI-tract, as enzyme supplementation did not further enhance $P$ availability. Network analysis, based on the abundance patterns of different microbial species in the gut, showed that interactions between microbes were becoming more complex with the use of enzymes and probiotics, potentially due to changes in substrate availability and $\mathrm{pH}$. Such results, together with an observed increase in abundance of beneficial gut microbes such as Lactobacillus and Bacillus species, indicate higher microbiota stability with positive impacts for gut health.

In the general discussion (Chapter 9), the effectiveness of phytase, NSPdegrading enzymes and probiotics in improving diet quality is outlined. In addition, insights for future research and the implications of research outcomes, for current and future fish and aquafeed production, were presented.

The main conclusions of the thesis are:

- NSP is not inert to digestion.

- Phytase improved the availability of $P$, calcium and crude ash fractions in the diet.

- Xylanase has the potential to improve nutrient digestibility.

- $\beta$-glucanase did not improve nutrient digestibility.

- Probiotic supplementation improved fat digestibility.

- Probiotics improved growth and reduced the energy requirements for maintenance.

- In Nile tilapia, the available $P$ requirement is greater than $7 \mathrm{~g} / \mathrm{kg}$ feed (dry matter).

- The combination of enzymes and probiotics enhanced microbial interactions in the distal gut.

- Enzymes, when supplemented to the diet, are mainly active in the stomach.

- The effect of enzyme supplementation is dependent on diet quality.

- Synergy between phytase and xylanase can occur.

Overall it can be concluded that, in Nile tilapia, the nutritional value of low quality diets was improved with exogenous enzyme and probiotic supplementation, resulting in enhanced growth performance. This implies that an increasing proportion of low quality plant-based co- and by-products could be incorporated into aquafeeds, thus contributing to the development of circular food systems. 


\section{Samenvatting}

Met de verwachte verdere groei van de aquacultuursector en de toenemende vraag naar visvoer, zal de dalende trend van de gehaltes aan vismeel en visolie in visvoer zich voortzetten. Het gebruik van laagwaardige ingrediënten in het voer van vele vissoorten, inclusief tilapia, zal in de toekomst verder toenemen. Deze veranderingen in de samenstelling van het voer leiden tot verhoogde niveaus van anti-nutritionele factoren, waaronder niet-zetmeel koolhydraten (NSP, beter bekend als vezels) en fytaat. Zowel NSP als fytaat worden gezien als onverteerbaar, omdat de enzymen die nodig zijn ze af te breken schaars zijn of ontbreken in vissen. Het toedienen van enzymen en probiotica kan de antinutritionele effecten van fytaat en NSP verminderen. In vergelijking met studies bij eenmagige landbouwhuisdieren, zijn studies bij vissen naar het gebruik van fytase en NSP-afbrekende enzymen als additief in voeders schaars. Eveneens zijn er meerdere onderzoeken uitgevoerd, bij zowel vleeskuikens als varkens naar de verbetering in nutriëntbeschikbaarheid door het gebruik van probiotica. Bij vissen zijn vergelijkbare onderzoeken beperkt; er is momenteel weinig bekend over hoe probiotica de beschikbaarheid van nutriënten in plantaardige visvoeders kan verbeteren. Daarnaast wordt verwacht dat er bij vissen synergie kan optreden tussen enzymen, en tussen enzymen en probiotica. Ook dit is echter nog niet onderzocht. Daarom was het algemene doel van dit proefschrift de potentie van enzym- en probioticatoevoeging te beoordelen ter verbetering van de voedingswaarde van visvoer met een lage kwaliteit. Bij dit onderzoek is Nile tilapia gebruikt als modeldier.

Allereerst is gekeken wat er bekend is in de literatuur over koolhydraten, in het bijzonder NSP, in Nile tilapia (Hoofdstuk 2). Naast een kwalitatieve aanpak, werd een meta-analyse uitgevoerd op beschikbare gegevens uit verteerbaarheidsstudies bij tilapia, waardoor kwantificering en schatting van de verteerbaarheid van koolhydraten en NSP mogelijk werd. Uit de meta-analyse bleek de gemiddelde NSP-verteerbaarheid $24.3 \%$ te zijn. Van alle onderzochte gevallen gaf $88 \%$ een positieve NSP-verteerbaarheid. Deze NSP-verteerbaarheid wordt in verband gebracht met fermentatie in de darm. De meta-analyse bevestigde het negatieve effect van NSP op de groei resultaten van tilapia en ook op de verteerbaarheid van nutriënten (eiwit, vet en energie). De vertering van NSP bleek bij te dragen aan de energieverteerbaarheid in tilapia.

In de volgende twee hoofdstukken (Hoofdstuk 3 en 4) werd onderzocht of enzymen (fytase en xylanase) de verteerbaarheid van NSP en fosfor (P) kunnen verbeteren en daardoor de groei kunnen verbeteren en of de impact van enzymen afhankelijk is van voer gerelateerde factoren. Hoofdstuk $\mathbf{3}$ onderzocht of het effect van enzymtoevoeging op de groei en verteerbaarheid van de nutriënten afhankelijk is van het type ingrediënt(en) in het voer. Dit werd gedaan door tarwezemelen, zonnebloemschroot of citruspulp toe te voegen aan een referentiedieet. Deze ingrediënten zijn rijk aan NSP maar verschillen in NSP 
samenstelling. De enzymen verbeterden de groei van de vissen die het tarwezemelen- en zonnebloemschootdieet kregen, terwijl vissen die het referentiedieet (laag in NSP) en citruspulpdieet (rijk aan pectines) kregen, niet profiteerden van de enzymtoevoeging. Significante interactie effecten op de groei, en op energie en P-verteerbaarheid, geven aan dat de effectiviteit van de enzymtoevoeging afhankelijk was van het gebruikte NSP rijke ingrediënt en dus de samenstelling van de NSP-fractie in het visvoer. Hoofdstuk 4 onderzocht of het effect van fytase en xylanase afhankelijk was van de kwaliteit van het visvoer (niveau aan NSP en fytaat). Het contrast in voerkwaliteit (twee diëten) werd gecreëerd door verschillende hoeveelheden van ingrediënten met een lage kwaliteit te gebruiken. Het effect van fytase op de groei en verteerbaarheid van de nutriënten bleek onafhankelijk van de kwaliteit van het dieet, terwijl het effect van xylanase op de (verbeterde) verteerbaarheid van nutriënten afhankelijk was van de kwaliteit van het dieet.

In zowel hoofdstuk $\mathbf{3}$ en $\mathbf{4}$ waren de enzymen fytase en xylanase effectief in het verbeteren van nutriëntbeschikbaarheid en groei. Bij vleeskuikens is synergie tussen enzymen waargenomen, en hoogst waarschijnlijk is dit ook het gval bij vissen. In hoofdstuk 5 werd het effect van fytase, xylanase evenals de interactie (synergie) tussen deze twee enzymen onderzocht. Synergie tussen fytase en xylanase bleek aanwezig met betrekking tot hun effect op groei. De groei van vissen op het controledieet (geen enzymen) en op het dieet met xylanase waren vergelijkbaar, terwijl vissen op het dieet met fytase een verbeterde groei vertoonden. Het effect van fytase werd versterkt wanneer deze gecombineerd werd met xylanase. Zowel xylanase als fytase verbeterden de verteerbaarheid van de nutriënten, maar in tegenstelling tot de groei was er geen significant interactieeffect (synergie) aanwezig op de nutriëntverteerbaarheid. Uit de stikstofbalans bleek dat het effect van de synergie van deze enzymen op groei voornamelijk ontstond door het significante effect van fytase en xylanase op de eiwitretentie. Hoofdstuk 6 onderzocht of $\beta$-glucanase, een ander enzym dat NSP afbreekt, de groei en verteerbaarheid van nutriënten kan beïnvloeden, en of het een synergie aangaat met fytase en xylanase. In deze studie waren echter geen effecten van de enzymen xylanase en $\beta$-glucanase, noch hun combinaties, op de groei zichtbaar. Alleen fytase verbeterde de verteerbaarheid van $P$, calcium en de ruwe as-fractie. Verder werd een vergelijking gemaakt van de resultaten van het tarwezemelendieet in een eerdere studie met zelfde dieet en zelfde enzymbehandelingen (Hoofdstuk 3) om een verklaring te kunnen vinden voor de minimale enzym effecten in hoofdstuk 6. De resultaten van de vissen gevoerd met de diëten die fytase en xylanase bevatten waren vergelijkbaar tussen de hoofdstukken. De controlebehandeling zonder enzymtoevoeging in hoofdstuk 6 had echter een lagere voederconversie, een hogere droog stof en NSPverteerbaarheid, in vergelijking met hoofdstuk 3. Dit suggereert dat met de tijd voeding gerelateerde factoren het controledieet beïnvloed hebben en daarmee de herhaalbaarheid van de studie. Het is aannemelijk dat in de huidige studie en andere studies, die geen verbetering van groei laten zien door het gebruik van 
enzymen, de controlediëten onvoldoende beperkt zijn in de beschikbaarheid van de nutriënten voor groei.

Probiotica worden veelal gebruikt om de gezondheid en weerstand van vissen te verbeteren. Bovendien is aangetoond dat probiotica de nutriëntbenutting en voederefficiëntie verhogen, wat resulteert in een verbeterde groei. Studies die zowel probiotica als enzymen combineren in visvoer, zijn echter schaars. Hoofdstuk 7 onderzocht de impact van enzymen, probiotica, evenals hun synergetisch effect. Zowel enzymen als probiotica verbeterden de groei wanneer ze afzonderlijk gebruikt werden. De combinatie van enzymen en probiotica toonde een interactie-effect op groei. Enzymen verbeterden de groei sterker dan probiotica, terwijl de combinatie van enzymen en probiotica de groei niet verder verhoogde. De enzymen verbeterden de verteerbaarheid van alle nutriënten, terwijl probiotica vooral de verteerbaarheid van de vet-fractie verbeterden. Bovendien verminderde de toevoeging van probiotica de energiebehoefte voor onderhoud. Zowel enzymen als probiotica hadden positieve effecten op de gemeten parameters, maar een synergie en ook additieve wisselwerking tussen enzymen en probiotica was hoofdzakelijk afwezig. Tijdens dezelfde studie werden monsters van de darm en chymus langs het gehele maagdarmkanaal verzameld. Deze werden gebruikt om het effect van enzymen en probiotica op de verteringskinetiek in het maagdarmkanaal te bestuderen, evenals hun invloed op de microbiota in de distale darm (Hoofdstuk 8). Verschillen in P-beschikbaarheid met enzymtoevoeging waren aanwezig in het proximale deel van het maagdarmkanaal. Dit geeft aan dat de enzymen actief waren (afbraak van fytaat) in de maag en mogelijk in het maagdarmkanaal, aangezien de enzymtoevoeging de P-beschikbaarheid na de maag niet verder verhoogde. Netwerkanalyse, gebaseerd op de aanwezigheids-patronen van verschillende microbiële soorten in de darm, toonde aan dat de interacties tussen microben complexer waren met toevoeging van enzym en probiotica, mogelijk als gevolg van verandering in de beschikbaarheid van substraat en $\mathrm{pH}$. Dergelijke resultaten, samen met de waargenomen toename in aanwezigheid van nuttige darmmicroben zoals Lactobacillus en Bacillus soorten, duiden op een hogere stabiliteit van het microbioom met positieve gevolgen voor de darmgezondheid.

In de algemene discussie (Hoofdstuk 9) wordt de effect op de kwaliteit van het voer van fytase, enzymen die NSP afbreken en probiotica toevoegingen uiteengezet. Daarnaast zijn inzichten voor toekomstig onderzoek en de implicaties van de onderzoeksresultaten voor de huidige en toekomstige vis- en visvoerproductie gepresenteerd.

De belangrijkste conclusies van het proefschrift zijn:

- NSP is niet inert voor vertering.

- Fytase verbetert de beschikbaarheid van $\mathrm{P}$, calcium en de as-fractie in het voer.

- Xylanase heeft de potentie om de nutriëntverteerbaarheid te verbeteren.

- $\beta$-glucanase verbetert de verteerbaarheid van de nutriënten niet. 
- Toevoeging van probiotica verbetert de vetverteerbaarheid.

- Probiotica verbeteren de groei en verminderden de energiebehoefte voor onderhoud.

- In Nile tilapia is de beschikbare P-behoefte hoger dan $7 \mathrm{~g} / \mathrm{kg}$ voer (op droge stof basis).

- De combinatie van enzymen en probiotica versterkt microbiële interacties in de distale darm.

- Enzymen zijn, wanneer ze aan de voeding worden toegevoegd, voornamelijk actief in de maag.

- Het effect van enzymtoevoeging is afhankelijk van de dieetsamenstelling.

- Er kan synergie optreden tussen fytase en xylanase.

In het algemeen kan worden geconcludeerd dat in Nile tilapia de voedingswaarden van diëten met een lage kwaliteit wordt verbeterd door het toevoegen van enzymen en probiotica, wat resulteert in een verhoogde groei. Dit suggereert dat er meer plantaardige co- en bijproducten van lage kwaliteit kunnen worden gebruikt dan momenteel gebruikelijk, en zodoende kan bijdragen aan de ontwikkeling van circulaire voedselsystemen. 



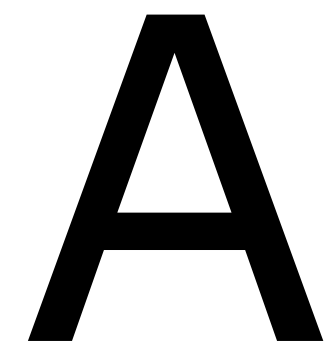

About the author

Completed training and supervision

Acknowledgements 


\section{About the author}

Roel M. Maas was born on $15^{\text {th }}$ January 1991, in Goes, The Netherlands. He received his HAVO degree from the Goese Lyceum in Goes. In his youth, growing up in Zeeland (Wolphaartsdijk), he was surrounded by water, which triggered his interest and fascination for water and the aquatic world. Hence, he chose to start his bachelor education in aquatic ecotechnology at the university of applied sciences (HBO) Hogeschool Zeeland ( $\mathrm{HZ}$ ), in Vlissingen, in 2008. During his BSc internship at CARMABI

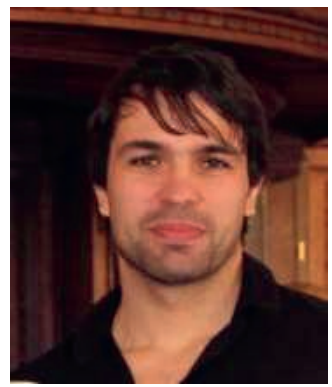
in Curacao, Dutch Caribbean, he mapped the fish population around the island by counting tens of thousands of fish during more than 100 dives. For his final internship he cultivated algae on a large scale, investigating the factors affecting its growth. After obtaining his bachelor degree he completed a MSc in Aquaculture and Marine Resource Management at Wageningen University (WU). After following a course on fish nutrition, he decided to orientate himself in this direction. His major thesis was on the effect of phosphorus requirements on diet composition (at WUR), with a minor thesis on the effect of oxygen levels on diet preference (at INRA, France), both studies using Rainbow trout. After he obtained his MSc degree in 2014, he started working at Hjatland Sea Farms Ltd. (Grieg Seafood) on the Shetland Isles in Scotland, in an Atlantic salmon hatchery as a fish husbandry technician. In October 2015, Roel started his NWO-funded PhD, "Green aquafeeds: unlocking non-starch polysaccharides to improve feed utilization and probiotic benefits", within the Aquaculture and Fisheries group (AFI) at WU, resulting in this thesis. His contract was extended during the last year of his PhD to perform a wide range of tasks within the AFI group as a Post-doctoral researcher. From January 2021, he continued working as an aquaculture researcher at AFI, with a focus on recirculating aquaculture systems (RAS) and the effects thereof on culture animals.

Contact: roel.maas@wur.nl 


\section{List of publications}

Maas, R.M., Verdegem, M.C.J., Dersjant-Li, Y., Schrama, J.W., 2018. The effect of phytase, xylanase and their combination on growth performance and nutrient utilisation in Nile tilapia. Aquaculture 487, 7-14.

Maas, R.M., Verdegem, M.C.J., Schrama, J.W., 2019. Effect of non-starch polysaccharide composition and enzyme supplementation on growth performance and nutrient digestibility in Nile tilapia (Oreochromis niloticus). Aquac. Nutr. 25, 622-632

Prabhu, P.A.J., Fountoulaki, E., Maas, R.M., Heinsbroek, L.T.N., Eding, E.H., Kaushik, S.J., Schrama, J.W., 2019. Dietary ingredient composition alters faecal characteristics and waste production in common carp reared in recirculation system. Aquaculture 512, 734357.

Maas, R.M., Verdegem, M.C.J., Wiegertjes, G.F., Schrama, J.W., 2020a. Carbohydrate utilisation by tilapia: a meta-analytical approach. Rev. Aquacult. 12, 1851-1866.

Maas, R.M., Verdegem, M.C.J., Stevens, T.L., Schrama, J.W., 2020b. Effect of exogenous enzymes (phytase and xylanase) supplementation on nutrient digestibility and growth performance of Nile tilapia (Oreochromis niloticus) fed different quality diets. Aquaculture 529, 735723

Maas, R.M., Verdegem, M.C.J., Chia-Ning Lee, Schrama, J.W., 2020c. Effects and interactions between phytase, xylanase and $\beta$-glucanase on growth performance and nutrient digestibility in Nile tilapia. Anim. Feed Sci. Technol. 271, 114767.

Maas, R.M., Verdegem, M.C.J., Sudip Debnath, Leon Marchal, Schrama, J.W., 2020d. Effect of enzymes, probiotics and their combination on growth performance and nutrient utilisation in Nile tilapia. Aquaculture 553, 736226.

Maas, R.M., Deng, Y., Dersjant-Li, Y., Petit, J., Verdegem, M.C.J., Schrama J.W., Kokou, F., Exogenous enzymes and probiotics alter digestion kinetics and microbial interactions in Nile tilapia gut (submitted manuscript). 


\section{Completed training and supervision}

The Basic Package

WIAS Introduction Day

WIAS Course on Essential Skills

Research Integrity and Ethics in Animal Sciences

\section{Disciplinary Competences}

Introduction to Laboratory Animal Science: Design and Ethics in Animal Experimentation

Laboratory Animal Science: Species Specific Course Fish

Knowledge exchange program AFI Japan

Writing a literature review

Advanced Statistics Course Design of Experiments

Knowledge exchange program AFI China
3 ECTS

2015

2015

2017

13.3 ECTS

2016

2016

2016

2017

2017

2019

\subsection{ECTS}

2016

2017

2017

2018

2019

2020

4 ECTS

2017

2018

WIAS Science Day, Wageningen, The Netherlands

(Oral presentation)

International Symposium on Finfish Nutrition and Feeding (ISFNF), 2018

Las Palmas de Gran Canaria, Spain (Poster presentation)

European Aquaculture Society (EAS) Conference, Berlin, 2019

Germany (Oral presentation)

WIAS Science Day, Lunteren, The Netherlands (Oral presentation) 
Teaching Competences

Supervising major MSc thesis: Thomas de Krom

Supervising BSc thesis: Marieke Swinkels

Supervising major MSc thesis: Racha Saksouk Dit Racha Sasso

Supervising major MSc thesis: Chia-Ning Lee

Supervising major MSc thesis: Theodor Stevens

Supervising major MSc thesis: Dita Wisudyawati Editya

Supervising major MSc thesis: Sudip Debnath

Supervising practical and tutorials
6 ETCS

2016

2016

2016-2017

2017

2017-2018

2018-2019

2019-2020

2016-2020

Education and Training total:

30.4 ECTS

Completion of the training activities in in fulfilment of the requirements for the education certificate of the Graduate School of the Wageningen Institute of Animal Sciences (WIAS). One ECTS equals a study load of 28 hours. 


\section{Acknowledgements}

It was a very long journey, but I made it! Now it is time to move on to the next chapter, but not before looking back and expressing my gratitude to everyone who was involved in my PhD, including in my personal life, Thank you! There are some people whom I would like to thank in particular.

Johan, after following your nutrition course, I found that I did not want to go diving (and relaxing on the beach) in the Dutch Caribbean anymore, but rather I wanted to feed fish inside a research facility. I'm still not sure how you did this.... anyhow, I never regretted this decision. To say that I learnt a lot from you (and continue to do so) is a big understatement. The idea of doing a PhD never crossed my mind until you asked me if I had ever considered it, the outcome of that discussion is now evident. You always made sure I was progressing and you were available to answer many of my "short" questions, despite your busy schedule. I still get frustrated with reviewers, but now I try to address them in a diplomatic way, instead of trying to convince them that their remark is stupid. Thanks for everything!

Marc, my second promotor, thank you for keeping a good balance within the project, it was valuable to have someone involved that looked at things from a different angle.

I would also like to thank Geert \& Johan V., both of you decided to hire me at some point (thanks for those opportunities) and came on a PhD trip (Japan, \& China), which was great. Johan, I hope to continue to see you around at conference, where going out for dinner with you always guarantees good food (except in Japan).

Yueming, my contact at DuPont, thank you for our enjoyable collaboration and for your valuable input. You were always easy to reach whenever I had a question or needed something.

I would like to express my gratitude to Menno and all the biotechnicians at Carus (Wian, Emily, Sander, Truus \& Tom), you were key in running the experiments and in the data collection, including long days of striping fish guts (sorry for that). Special thanks to Wian and Emily, who acted as first responsible biotechnicians during my experiments. Thanks to the lab (Ronald, Tino, Erik, Samara) for help with analysing large quantities of samples. Especially Tino, who guided most of my students in the lab, and Erik for his help with the gut microbiota and sampling.

Annet, Marjon \& Eugenia, life at AFI was so much easier knowing you had all the answers when I got lost in the university system, or whenever I had a "stomme vraag". Miranda, your involvement started when I was dealing with a demanding reviewer, but by now you have probably read most of my thesis. Your input is highly appreciated! I learnt a lot from it. Ep, it was always nice to talk about phosphorus and RAS (will be continued!). Jules, thanks for your help (and pipetting skills) with the lactate measurements. To all the other AFI staff members (Geertje, Fotini, Maria, Esther, Leo, Paul, Jan-Jaap), thank you all for the nice atmosphere at work.

To my students: Thomas, Marieke, Racha, Jennifer (Chia-Ning), Theodor, Dita \& Sudip, thank you for all your hard work and input! You saved me many hours by feeding our fish, but above all your out-of-the box questions and thesis reports kept me on my toes and were a good source of input. 
Special thanks to my fellow-PhD candidates that joined me (or I joined them) on this PhDadventure: Thomas, Twan, Marit, Kim, Davood, Yale, Alam, Zhang, Joost, Vivi (my KLM...), Vinna, Tu, Kabir, Tihn, Thuat, Gauthier, Elisa, Peter, Annemiek, Timo, Christos, Mahmoud, Devi \& Folasade. Please forgive me if I missed out one of you. Thomas, we started on the same day and our PhD's took a similar course, it was good to have someone to go to conferences with that had a similar interest in talking to strangers. Thanks for the many coffee (water for you) breaks, random talks and advices. All the best in Zeeland (best province to be honest), luckily I know my way around there. Davood (Karimi as I would say :P), I spent most time with you in E0232. We've had our fair share of discussions about things that don't even really matter. But above all, we really understood each other using just a few words $\left(\mathrm{CO}_{2}\right.$, or not even a word). Marit, it took a few years to realise that you are not that scary. Thanks for all the chats (about basically everything, but often fish poop) that always took longer than anticipated. I hope I still can be of use as your "hulplijn" for many years to come (and vice versa). Twan, although our ideas contrast in many ways (good experimental designs (tanks) vs. ...), it clicked from the start. Thanks for being my "paranymph", for the occasional beers and the beers to come.

Solomon, Amelie Lens, Carl Cox, Peggy Gou, Deborah de Luca, Objekt, Nina Kravitz, PanPot, Polo Pan, Acid-Pauli, Stephan Bodzin, Charlotte de Witte, La Fleur, Manu Le Tough, Artbat, Boris Brejcha and many more, thanks for the music you created! I hardly wrote anything without you blasting through my headphones.

Luckily there were my AET mates, my Master's "fish friends" (Tim, Jos \& Thibaut, soon we really will go on a trip) and my life-long buddies in Zeeland, that took my mind off my PhD sometimes. Tim, bedankt voor de wekelijkse lunches toen je nog bij entomologie zat, de latere bezoekjes in Gorinchem of Bennekom en de garnalen. Ook al hebben we samen vaak de neiging te mopperen, het is wel altijd gezellig. Fijn dat je mijn paranimf wil zijn.

Lieve Papa en Mama (Vatie \& Moesie), jullie staan aan de basis van waar ik nu sta. Jullie hebben me altijd gesteund en achter mijn keuzes gestaan, ook toen ik niet naar het VWO wou omdat "ik toch niet ga studeren aan de universiteit" of bedacht voor onbepaalde tijd naar Shetland te vertrekken. Altijd fijn om weer in Zeeland te zijn, waar jullie altijd goed voor mijn chickies gezorgd hebben en waar ik een tweede thuis heb, want jullie weten "diep in mien arte bin ik ok voe Wolfersdiek". Dank jullie voor alles!

Rob \& Charlotte, fijn m'n jeugd met jullie te hebben mogen delen, jullie weten als geen ander hoe het is een Maasje te zijn. Rob, toen ik in Wageningen ging studeren waren velen verbaast dat ik bij jou op de gang (9A) ging wonen en in dezelfde ploeg ging roeien, ik vond het vooral erg gezellig. Fijn dat jij en Annemiek (bedankt voor je hulp met de Nederlandse samenvatting) ook tijdens m'n PhD nog een tijdje in de buurt woonden zodat we onder andere samen (vooral met Annemiek) BZV konden kijken. Het gaat jullie (Mark \& Sam natuurlijk ook) goed!

Lieve Ivy, de eerste maand van mijn PhD leerden ik je kennen, sindsdien is het snel gegaan. Bedankt voor de welgemeende interesse (wederzijds wordt dat steeds lastiger met je IT carrière), het aanhoren van mijn geklaag, het tolereren van mijn vis gerelateerde geurtjes en soms rare werktijden. Maar vooral bedankt voor het creëren van een thuis (met Lola), waar het fijn thuiskomen is na een lange dag, waar ik kan ontspannen, mezelf kan zijn en tegen jou kan aankruipen op de bank. Ik hou van jou!

"All I can do is be me, whoever that is" - Bob Dylan

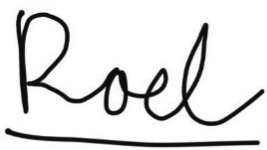




\section{Colophon}

The research described in this thesis was financially supported by The Netherlands Organisation for Scientific Research (NWO). The project is entitled "Green aquafeeds: unlocking non-starch polysaccharides to improve feed utilization and probiotic benefit", project number 870.15.100. DuPont Animal Nutrition partly funded the NWO project.

Financial support from Wageningen University for printing this thesis is gratefully acknowledged.

Image tilapia: FAO FishFinder, the Species Identification and Data Programme Cover design: Ivy Visser

Printed: ProefschriftMaken | www.proefschriftmaken.nl on recycled, FSC certified paper 

